Florida International University FIU Digital Commons

6-1-2017

\title{
The Identification of Volatile Organic Compounds from Synthetic Cathinone Derivatives for the Development of Odor Mimic Training Aids
}

Vanquilla Shellman Francis

Florida International University, vshel001@fiu.edu

DOI: $10.25148 /$ etd.FIDC001965

Follow this and additional works at: https:// digitalcommons.fiu.edu/etd

Part of the Analytical Chemistry Commons

\section{Recommended Citation}

Francis, Vanquilla Shellman, "The Identification of Volatile Organic Compounds from Synthetic Cathinone Derivatives for the Development of Odor Mimic Training Aids" (2017). FIU Electronic Theses and Dissertations. 3370.

https://digitalcommons.fiu.edu/etd/3370 


\section{FLORIDA INTERNATIONAL UNIVERSITY}

Miami, Florida

THE IDENTIFICATION OF VOLATILE ORGANIC COMPOUNDS

FROM SYNTHETIC CATHINONE DERIVATIVES

FOR THE DEVELOPMENT OF ODOR MIMIC TRAINING AIDS

A dissertation submitted in partial fulfillment of the

requirements for the degree of

DOCTOR OF PHILOSOPHY

in

CHEMISTRY

by

Vanquilla Shellman Francis 
To: Dean Michael R. Heithaus

College of Arts, Sciences and Education

This dissertation, written by Vanquilla Shellman Francis, and entitled The Identification of Volatile Organic Compounds from Synthetic Cathinone Derivatives for the Development of Odor Mimic Training Aids, having been approved in respect to style and intellectual content, is referred to you for judgment.

We have read this dissertation and recommend that it be approved.

$\begin{array}{r}\hline \text { Norman Munroe } \\ \hline \text { John Berry } \\ \hline \text { Watson Lees } \\ \hline \text { Kenneth G. Furton, Major Professor }\end{array}$

Date of Defense: June 1, 2017

The dissertation of Vanquilla Shellman Francis is approved.

Dean Michael R. Heithaus

College of Arts, Sciences and Education

Andrés G. Gil

Vice President for Research and Economic Development

And Dean of the University Graduate School

Florida International University, 2017 
C Copyright 2017 by Vanquilla Shellman Francis

All rights reserved. 


\section{ACKNOWLEDGMENTS}

First and foremost I would like to thank God, for without Him none of the work and success I have had thus far would have been achieved.

I would like to say thank you to my parents Yulanda and Nelson Shellman. Thank you for all the love and guidance you have given me since I entered into this world. Thank you for always believing in me even when I didn't believe in myself. Thank you for being the shoulders I needed to cry on and my direct line to Christ when I felt like giving up. Words can never truly express how blessed I am to have such amazing parents like you guys. For Dr. Tiana Coleman, from the moment I entered into your classroom in the $8^{\text {th }}$ grade, you and Mrs. Cabrera have continuously held my hand and encouraged me to strive for greatness. You are more than a mother figure to me, you are a voice of reason in the midst of any storm and I thank you for continuously being there throughout the years. To my siblings (Alexis, Vanessa, and Chris) I miss you all more than words can express. It has been hard being without my best friend (Alexis) and being so far from the people I have known all my life. I thank you guys for the love and support and always keeping me reminded of who I truly am.

To the man that has changed my life for the better, my husband Jeffery Francis, I love you. From the first moment we met in our biochemistry class at FIU to this very moment you have been by my side. You have been the true definition of a great support system, a rock, and an oasis. You have sacrificed so much for me to get to this point in my life. You continuously lift me up when I have fallen, correct me when I am wrong, and continuously awake the true meaning of happiness within me. Obtaining this 
doctorate would not have been possible with you and my parents. I cannot wait to begin the next chapter of our lives together and I pray that the years to come only bring us closer and we will be able to achieve great things.

To my fellowships, McKnight and McNair, thank you for supporting me throughout all my endeavors. To my second family, the Furtonettes, where should I begin? I would like to thank Iris for taking me under her wing and showing me how to be a great scientist. You taught me how to conquer the beast known as the Varian. To Adhly, you are truly more than a colleague; you are my sister. You have been my family away from my family, my home away from my home. We walked together through this journey and I am grateful to know that our journey doesn't end here. To Rodolfo and Philip, the two of you are amazing. You guys have made this journey so much more bearable and enjoyable. Thank you for all the laughter and support throughout the years. To my unwanted big brother, Howard Holness, thank you. You have pushed me when I didn't want to be pushed. You have challenged me into bettering myself not only as a person but as a scientist. You truly deserve your own day to be recognized for all you continue to do. Dr. Kenneth G. Furton, thank you for forcing me into this doctoral program when I first met you 7 years ago. Thank you for seeing something in me that I didn't see in myself at that time. Lastly, to the babies in the lab, Alice and Kelvin, I am honored to have gotten the chance to teach you, scold you, and laugh with you over the past two years. I have no doubt you will both will conquer this program and continue on the traditions and integrity set forth in this lab. 


\author{
ABSTRACT OF THE DISSERTATION \\ THE IDENTIFICATION OF VOLATILE ORGANIC COMPOUNDS \\ FROM SYNTHETIC CATHINONE DERIVATIVES \\ FOR THE DEVELOPMENT OF ODOR MIMIC TRAINING AIDS \\ by \\ Vanquilla Shellman Francis
}

Florida International University, 2017

Miami, Florida

Professor Kenneth G. Furton, Major Professor

Methylone, Ethylone, Methylenedioxypyrovalerone (MDPV), and $\alpha$ Pyrrolidinopentiophenone ( $\alpha$-PVP) collectively referred to as bath salts are a new trend of illicit substances known as synthetic cathinones. Designed by chemically modifying the core structure of the compound cathinone, synthetic cathinones became prevalent within the United States around the mid-2000s. As a cheap and less controlled alternative to methylenedioxymethamphetamine (MDMA, ecstasy), it has become heavily abused, prompting emergency scheduling by federal regulators. Although regulations have been placed to halt incoming traffic of these drugs, lack of ground efforts still leave a large percentage of bath salts available.

This study is two-fold, as it seeks to develop an extraction method for the development of Volatile Organic Compound profiles associated with various synthetic 
cathinones; and also determine the odorant used for canine recognition. The initial goal of this dissertation was to develop an extraction method to characterize various cathinone derivatives. The present study concluded that by employing a Polydimethylsiloxane Divinylbenzene (PDMS/DVB) coated fiber along with complimentary soft ionization techniques, the volatile components and all parent drugs could be identified within sixteen synthetic cathinone cases.

The second goal of the dissertation was to assess and enhance the detection capabilities of narcotic detection teams. Canine field detection is routinely used to stop the increasing distribution influx of drugs into the United States that go undetected by standard procedures currently employed. Although currently canines can detect a multitude of drugs including heroin, cocaine, MDMA, and methamphetamine; this study revealed that more than ten canine teams (throughout south Florida) were not able to detect the presence of synthetic cathinones with current training material.

While concerns have grown concerning the safety and reliability of canines being trained on various illicit substances, this research aimed to develop a safe, efficient, controlled alternative to training any canine for detection in the form of a Controlled Mimic Permeation System (COMPS). Field examination concluded that 3,4methylenedioxypriophenone was the odorant responsible for the canine recognition of the cathinone derivative odor. Therefore a mimic training aid was developed and deployed within the field to enhance the detection capabilities of various canine teams. 


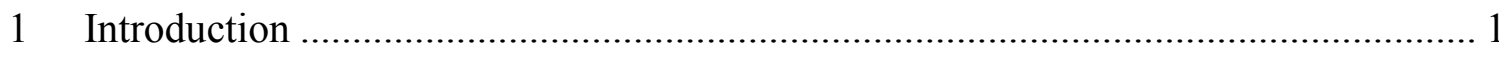

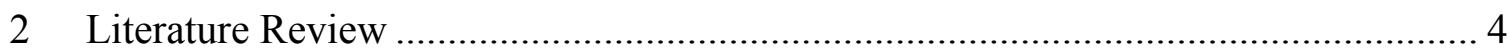

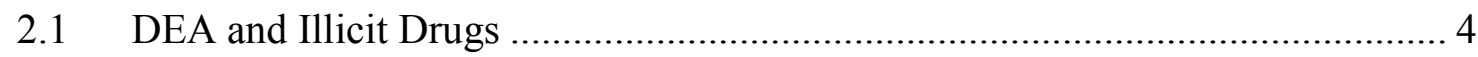

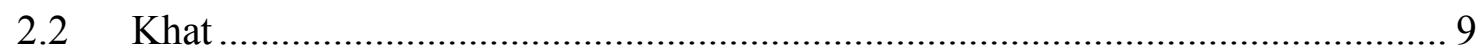

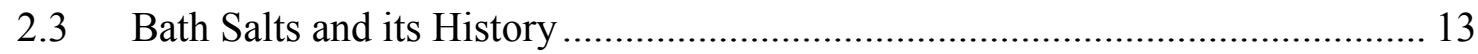

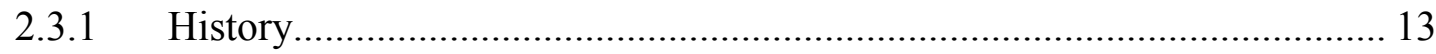

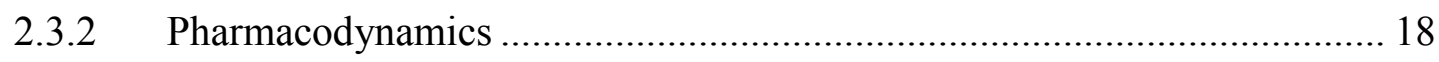

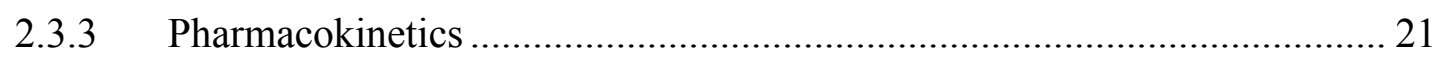

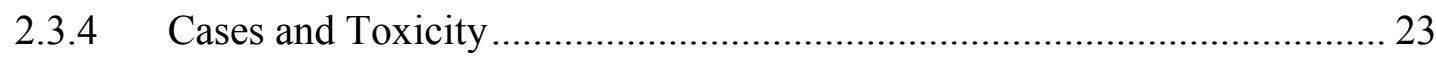

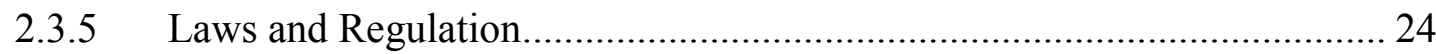

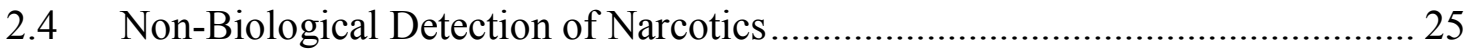

2.4.1 Color/Spot Testing .......................................................................... 25

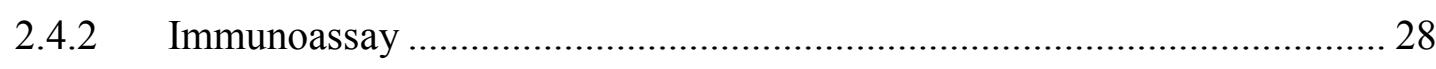

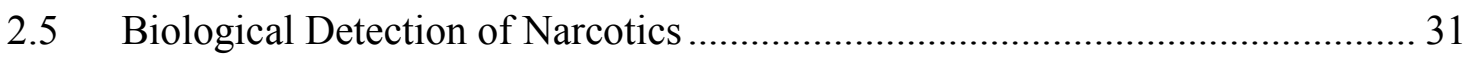

2.5.1 Canine Detection History........................................................................ 31

2.5.2 Canine Olfaction and Mechanism............................................................ 32

2.5.3 Canine Training ................................................................................. 35

2.5.4 Current Hazards to Canines While Training ............................................... 37

2.6 Controlled Odor Mimic Permeation System ..................................................... 38

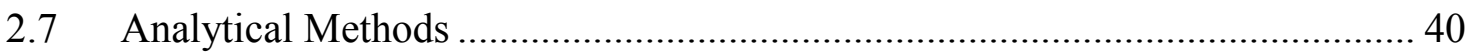

2.7.1 Solid Phase Microextraction ....................................................................... 40

2.7.2 Gas Chromatography ………………………….................................. 47

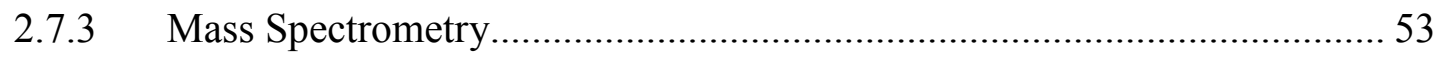

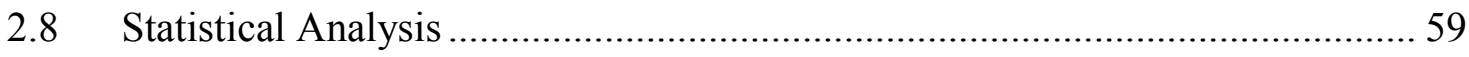

2.8.1 Statistical Significance Test (T-test) ……………..................................... 59

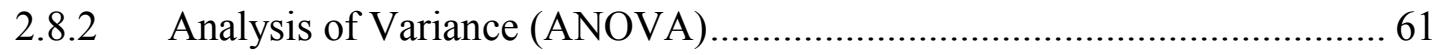

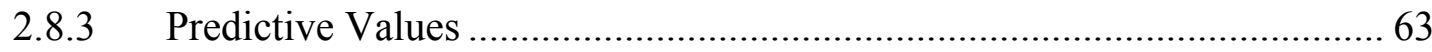




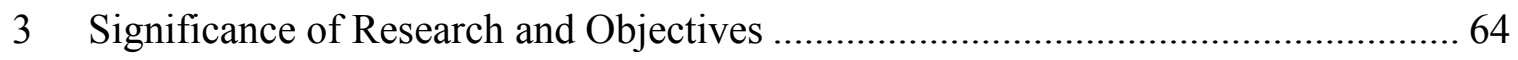

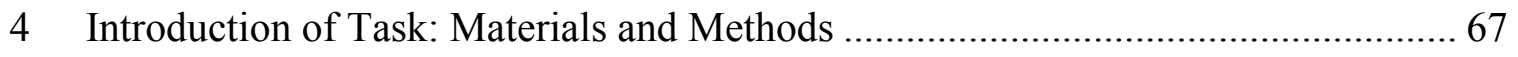

4.1 Analytical Method Optimization for the Detection of Volatile Organic Compounds Released from Synthetic Cathinones ........................................................ 67

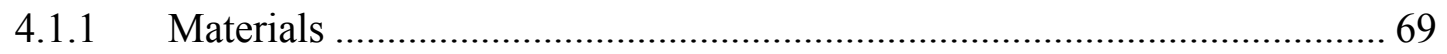

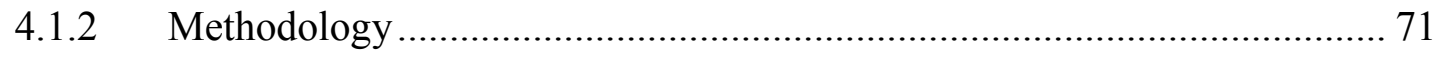

4.2 HS-SPME Method Optimization for the Detection of Volatile Organic Compounds Released from Synthetic Cathinones ……………………....................... 75

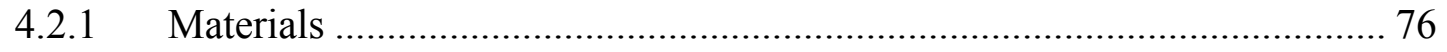

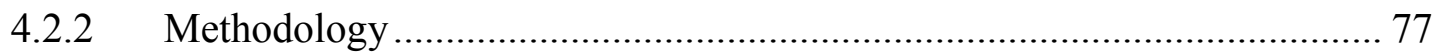

4.3 Assess the Volatile Organic Compounds Released from Synthetic Cathinones 78

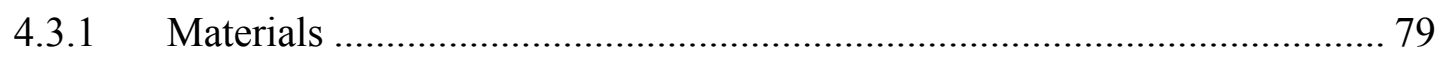

4.3.2 Methodology …………………………………................................ 79

4.4 Imprinting of Canines to Detect Illicit Bath Salts .............................................. 81

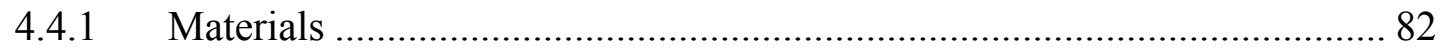

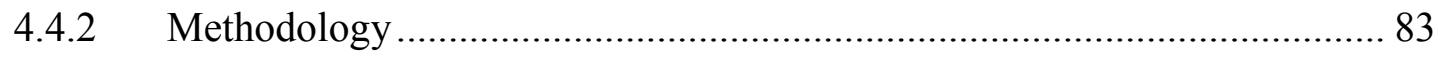

4.5 Isolate the Volatile Oragnic Compound(s) in Synthetic Cathinones Detectable by Ceritified Canines.................................................................................................. 89

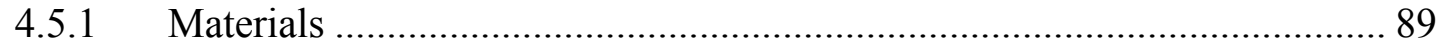

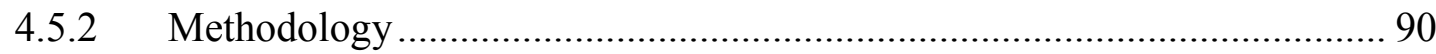

4.6 Optimization of the Controlled Odor Mimic Permeation System for Synthetic

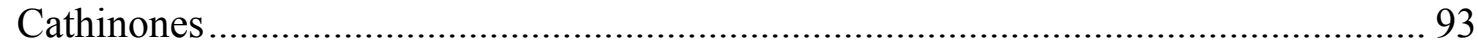

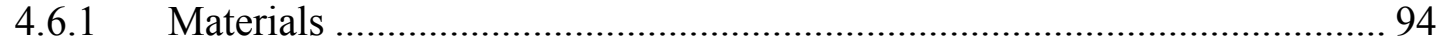

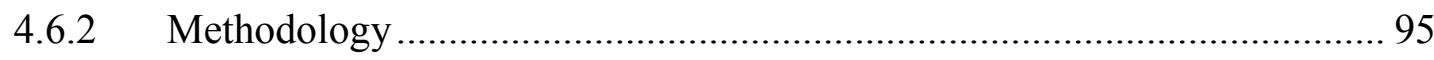

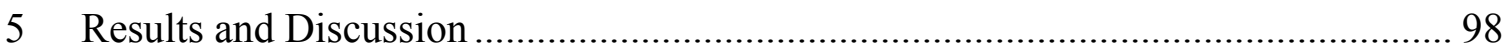

5.1 Analytical Method Optimization for the Detection of Volatile Organic Compounds Released from Synthetic Cathinones ...................................................... 98

5.1.1 Parameter and Method Optimization ......................................................... 98

5.1.2 Development of a Chemical Ionization Mass Spectrometry Method....... 108

5.2 HS-SPME Method Optimization for the Detection of Volatile Organic

Compounds Released from Synthetic Cathinones ……………………...................... 138

5.2.1 Optimal Fiber Selection for Headspace Solid-Phase Microextraction (HS-SPME). 
5.2.2 Optimal Extraction Parameters for HS-SPME ....................................... 139

5.3 Assess the volatile organic compound released from synthetic cathinones ..... 155

5.4 Imprinting Canine to Detect Synthetic Cathinones.......................................... 169

5.4.1 Detection of Synthetic Cathinones via Currently Deployed Narcotic

Detection Canine Teams..................................................................................... 169

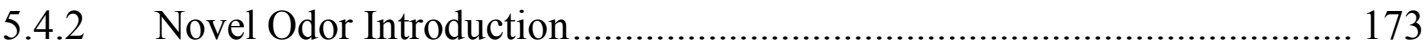

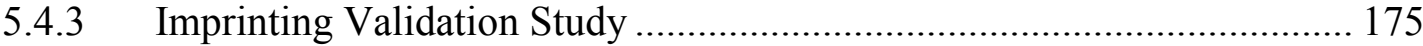

5.5 VOC Isolation for Development of a Controlled Odor Mimic Permeation

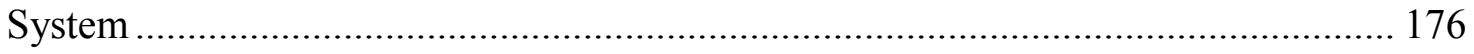

5.5.1 Development of Organic Compound Delivery System ………………..... 176

5.5.2 Compound Introduction to Canines for the Determination of the Active Odorant 178

5.6 COMPS Optimization for Synthetic Cathinones ........................................... 185

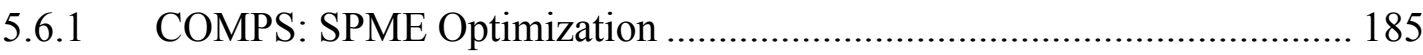

5.6.2 COMPS: Externally Sampled Internal Standard (ESIS) Optimization..... 188

5.6.3 COMPS: Absorbent Optimization ……………………………………..... 192

5.6.4 COMPS: Polymer Thickness Optimization .............................................. 197

5.6.5 COMPS: Containment and Validation....................................................... 201

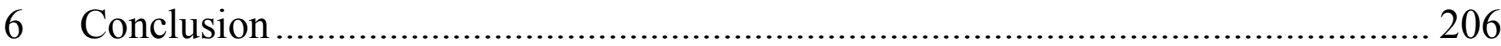

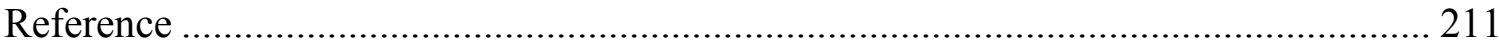

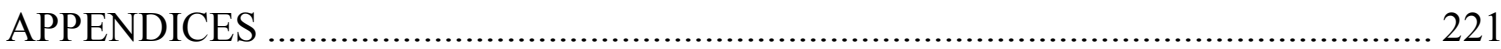

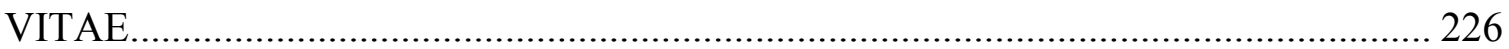




\section{LIST OF TABLES}

TABLE

PAGE

Table 1.Drug Enforcement Administration's Definition for Narcotic Scheduling and

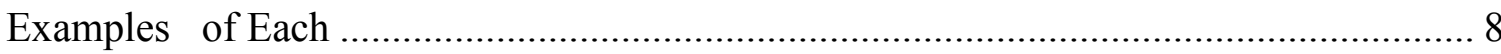

Table 2. Case Reports of Toxicity from Synthetic Cathinone Use ................................... 23

Table 3. Final Colors Produced when Chemical Reagents React with Various Drugs. *involved three separate stages of reaction producing three separate color results ${ }^{56}$....... 26

Table 4. The Commercially Available Fiber Coatings and Their Ideal Analyte Type

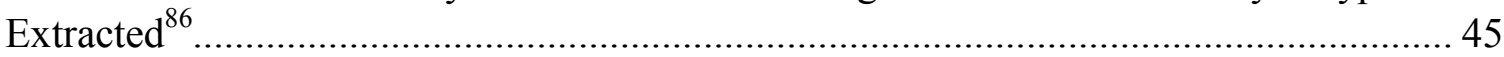

Table 5. Types of Column Stationary Phases used in Gas Chromatography ................... 52

Table 6. Types of Mass Spectrometric Analyzers .........................................................57

Table 7. Standards Purchased from Vendors ................................................................ 70

Table 8. Chemical Reagents used to determine the Optimal Chemical Ionization

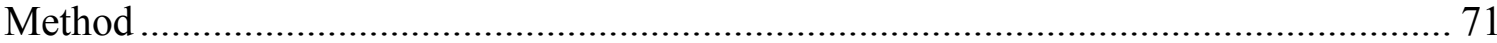

Table 9. Six Fiber Chemistries used to determine the Potential Number of Compounds

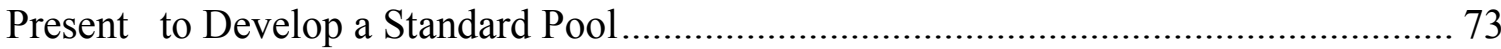

Table 10. VOC Comparison of Methylone, FHP MDMA Sample, and Literature Findings of Volatiles from MDMA Samples................................................................. 106

Table 11. VOC Comparison of Methylone, FHP Methamphetamine Sample, and Literature Findings of Volatiles from Methamphetamine Samples................................ 107

Table 12. Chemical Ionization (Ammonia) Parameter Modification of Storage Level and Ejection Amplitude

Table 13. Chemical Ionization (Ammonia) Parameter Modification of Background Mass (BM)

Table 14. Chemical Ionization (Ammonia) Parameter Modification of Maximum Ionization Time (Max IT)... 
Table 15. Chemical Ionization (Ammonia) Parameter Modification of Maximum

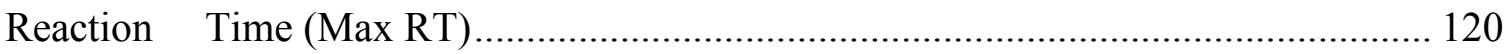

Table 16. Chemical Ionization (Methanol) Parameter Modification of Storage Level and Ejection Amplitude.

Table 17. Chemical Ionization (Methanol) Parameter Modification of Background Mass

Table 18. Chemical Ionization (Methanol) Parameter Modification of Maximum Ionization Time (Max IT).

Table 19.Chemical Ionization (Methanol) Parameter Modification of Maximum Reaction Time (Max RT).

Table 20.Chemical Ionization (Acetonitrile) Parameter Modification of Storage Level and Ejection Amplitude

Table 21. Optimum Settings for the Chemical Ionization Method................................ 135

Table 22. Scientific Names for the Compounds Discovered in the Extraction Process (Figure 35)

Table 23. Optimum Headspace-Solid Phase Microextraction Method for the Collection of VOCs from Synthetic Cathinones

Table 24. The Organic Compounds Detected Within the Headspace of Various Synthetic Cathinone Samples

Table 25. Detection Capabilities of Narcotic Detection Teams Deployed in Miami-Dade County 170

Table 26. Detection Capabilities of Narcotic Detection Teams Deployed in Palm Beach County 171

Table 27. Validation Test for Newly Imprinted Canines on Synthetic Cathinones........ 175

Table 28. Compound Exposure List for Active Odor Determination. 179

Table 29. Canine Alert Response Tabulated from Various Odor Delivery Bags 182 
Table 30. Canine Alert Response Tabulated from the COMPS Bags Containing

Table 31. Preliminary Field Surveillance of Detection of Synthetic Cathinones using Biosensors Imprinted on a Pseudo COMPS

Table 32. Chemical Ionization (Acetonitrile) Parameter Modification of Background

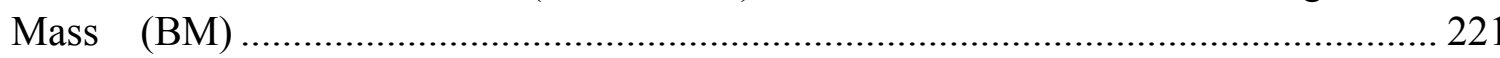

Table 33. Chemical Ionization (Acetonitrile) Parameter Modification of Maximum Ionization Time (Max IT). 


\section{LIST OF FIGURES}

FIGURE

PAGE

Figure 1. Khat plant ${ }^{1}$ 9

Figure 2. The Active Components of the Khat Plant: Cathinone, Cathine, and Norephedrine.

Figure 3. The Molecular Structure of the First Synthesized Cathinones, Ephedrone and Mephedrone

Figure 4. The Annual Reporting of Cases Called into the American Association of Poison Center with Questions in Regards to the Exposure of Synthetic Cathinones (Bath Salts) ${ }^{34}$

Figure 5. The Packaging of Bath Salts Sold in Headshops (Left $)^{41}$ and the Appearance of the Drug which May be Housed in These Packages (Right). 18

Figure 6. The Phases of Neurochemistry: The First Phase is where the Neurons communicate regularly through the Promotion of the Action Potential (A). The Second Phase the Drug of Question Blocks the Transporter Permitting the Reuptake of Certain Monoamines (B) The Third Phase the Drug Blocks the Uptake and Releases More Monoamines (C).

Figure 7. The Backbone Structure of Synthetic Cathinones. The R groups are Active Sites where the Molecule can be built on to Create New Derivatives of the Drug

Figure 8. Competitive ELISA where the Bound Antigen or Primary Antibody are Introduced to each Followed by Incubation and Wash. Afterwards an Enzyme Conjugate is added, Incubation and the Washing Phase are Repeated Before a Substrate in Introduced to Bind with the Enzyme Present to Induce a Color Change ${ }^{64}$.....

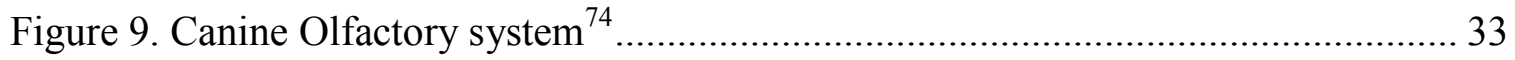

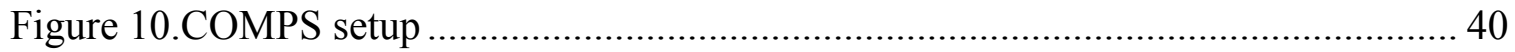

Figure 11. Solid Phase Microextraction (SPME) Fiber Assembly ................................ 41

Figure 12. Gas Chromatography Apparatus Configuration........................................ 49 
Figure 13. Mass Spectrometry Apparatus Configuration 53

Figure 14. The Schematic of the Miami-Dade Canine Trial ......................................... 84

Figure 15. Schematic of Palm Beach Canine Trial ...................................................... 86

Figure 16. Schematic of the Trial Setup at the Palm Beach off-site Airport Facility...... 92

Figure 17. Reference Chromatogram for the Extraction Completed with a Mason jar.. 100

Figure 18. Representative Sampling Device Contains for the Preliminary Extraction

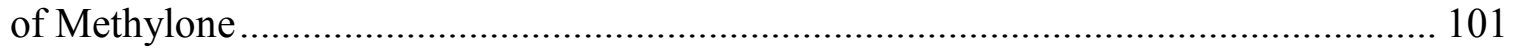

Figure 19. Comparison of the Compounds Extracted when the Container Volume is

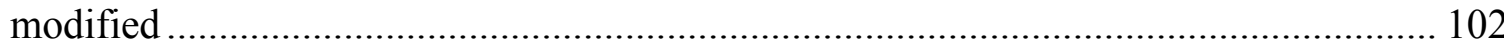

Figure 20. Peak Resolution of Methylone and MDPV on a Non-Polar Column............ 104

Figure 21. A. The Mass Spectrum of Methylone under Electron Ionization Mode.

B. The Mass Spectrum of Methylone under Chemical Ionization Mode ...................... 109

Figure 22. CI-Ammonia: Storage Level and Ejection Amplitude Parameter Optimization 113

Figure 23. Indistinguishable Mass Spectrum for 4-Bromo-1,2(methylenedioxy) benzene Operated under the Default Method of Ammonia 113

Figure 24. CI-Ammonia: Background Mass Parameter Optimization 116

Figure 25. Molecular Spectrum Obtained for Methylone under the Parameters of Method 15 .....

Figure 26. CI-Ammonia: Maximum Ionization Time Parameter Optimization 119

Figure 27.CI-Ammonia: Maximum Reaction Time Parameter Optimization ..... 121

Figure 28. Mass Spectrum of 1,3-Benzodioxole Detected within Method 6................ 123

Figure 29. Mass Spectrum of 1,3-Benzodioxole Detected within Method 9. 124

Figure 30.CI-Methanol: Storage Level and Ejection Amplitude Parameter Optimization 125 
Figure 31. Quantitative Comparison of the Optimal Chemical Ionization Reagent needed for Detection of Synthetic Cathinones

Figure 32. Fragmentation Pattern of Four Compounds Ionized by (a) Electron Impact Mode (b) Chemical Ionization Mode; 1) 1,3-Benzodioxole, 2) 4-

Bromo(methylenedioxy)benzene, 3) Methylone, 4) MDPV

Figure 33. HS-SPME Fiber Chemistry Comparison of the Extraction of the Target Compounds Potentially Associated with Synthetic Cathinones

Figure 34. Headspace Extraction Collected at Room Temperature vs an Elevated

Temperature $\left(50^{\circ} \mathrm{C}\right)$

Figure 35. Visual Observation on the Variation of Extractions Times and its Effect on the Collection of VOCs....

Figure 36. Variation of Times to Establish Equilibrium within a given Volume for Extraction

Figure 37. Variation of Temperatures for the Optimal Promotion of VOC Collection.. 150

Figure 38. Variation of GC Inlet Exposure for the Desorption of all Extracted VOCs.. 152

Figure 39. Assessment of the Analytes Retained on the PDMS/DVB fiber after the Initial Desorption

Figure 40. Visualization of Case 1 155

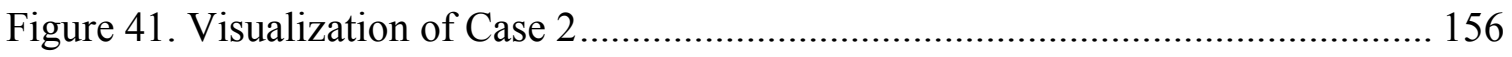

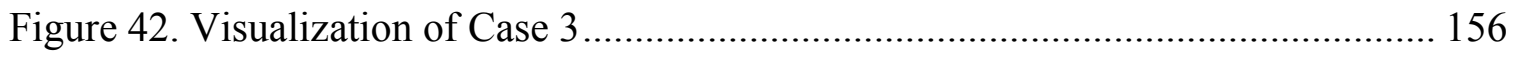

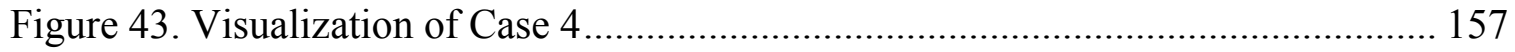

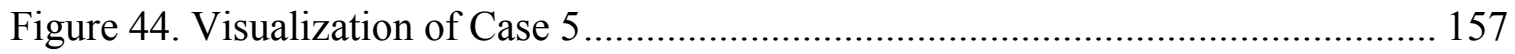

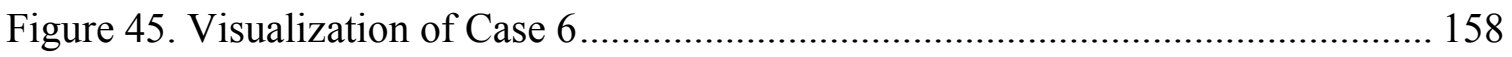

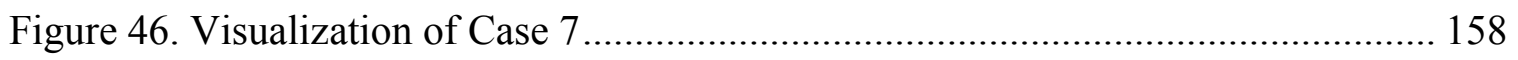

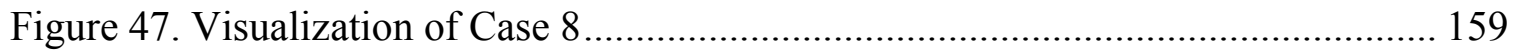




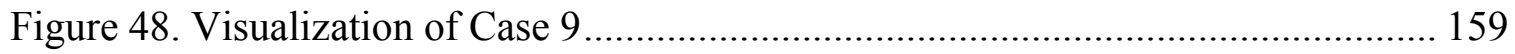

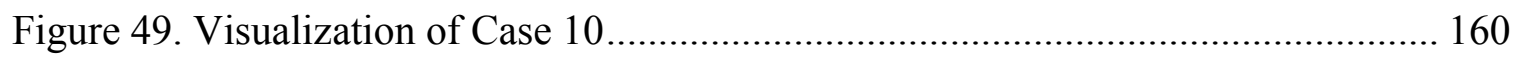

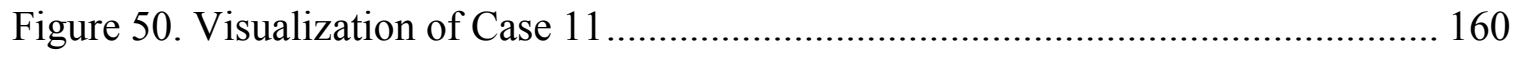

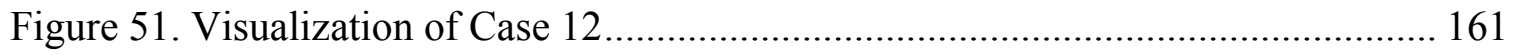

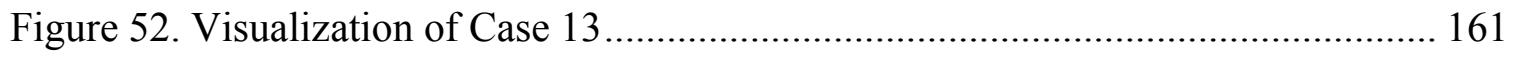

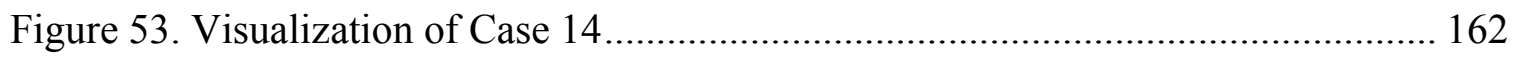

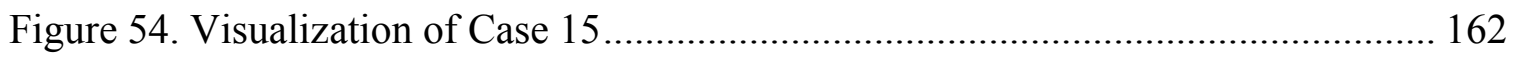

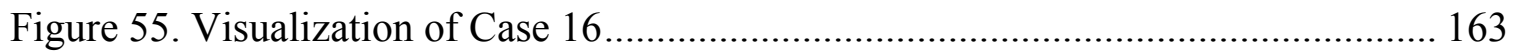

Figure 56. The Volatile Organic Compound (VOC) Profile of Sixteen Different Confiscated Synthetic Cathinones using the Optimal Headspace-Solid Phase Micro-

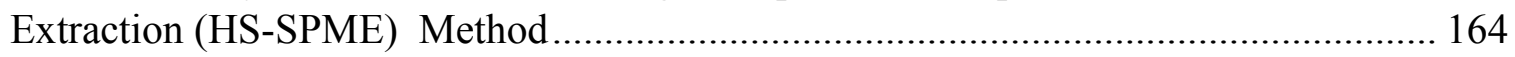

Figure 57. Cutting Agents Identified within the VOC Profiles of Analyzed Synthetic

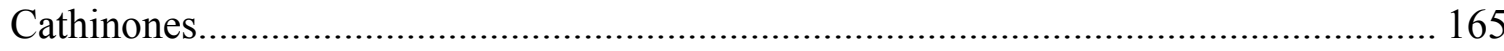

Figure 58. The Allotted Time Required for the Complete Dissipation of Methylene Chloride from a Surgical Gauze Pad ................................................................. 178

Figure 59. Reassessment of Various Equilibration Times Established for Development of the Optimal Synthetic Cathinone COMPS............................................ 186

Figure 60. Reassessment of Various Extraction Times Established for Development of the Optimal Synthetic Cathinone COMPS …………………..................................... 187

Figure 61. Assessment on the Effect Varying the Equilibration Time has on the Extraction of the Externally Sampled Internal Standard (ESIS)

Figure 62. Selection of the Optimal Extraction time for the Externally Sampled Internal Standard based on its Effect on the A/E Ratio versus Solely Observing the Analyte.

Figure 63. The Physical and Molecular Depiction of Various Absorptive Materials (a) Alumina (b) Cellulose and (c) Gauze Pad 193 
Figure 64. The Effect of Varying Absorbent Material on the COMPS Odor

Dissipation

Figure 65. The Dissipation Rate of Various Synthetic Cathinone COMPS Containing Different Phases

Figure 66.The Effect of Varying the Polymer's Gauge on the COMPS Odor

Dissipation

Figure 67. Temperature and Humidity Recordings during Optimization of the COMPS Polymer Gauge

Figure 68. Assessment of the Aluminum Bag as an Efficient Barrier Bag between the

Permeable Bag and the Surrounding Atmosphere 202

Figure 69. CI-Methanol Background Mass Parameter Optimization ............................. 222

Figure 70. CI-Methanol: Maximum Ionization Time Parameter Optimization............... 222

Figure 71. CI- Acetonitrile: Storage Level and Ejection Amplitude Parameter Optimization 223

Figure 72. CI Acetonitrile: Background Mass Parameter Optimization......................... 223

Figure 73. CI-Acetonitrile: Maximum Ionization Time Parameter Optimization .......... 224

Figure 74. Canine Odor Recognition Evaluation Form .................................................. 225 


\section{ABBREVIATION AND ACROYNMS}

3,4-DMMC

5-HT

ANOVA

BSD

CAR/PDMS

CI

COMPS

CNS

DA

DART

DAT

DEA

DVB/CAR/PDMS

EI

EIA

ELSIA

EM

$\mathrm{EPH}$

FHP

FIPA

GC
3,4-Dimethylmethcathinone

Serotonin

Analysis of Variance

Behavioral Shaping Device

Carboxen/Polydimethylsiloxane

Chemical Ionization

Controlled Odor Mimic Permeation Systems

Central Nervous System

Dopamine

Direct Analysis in Real Time

Dopamine Transporter

Drug Enforcement Agency

Divinylbezene/Carboxen/

Polydimethylsiloxane

Electron Ionization

Enzyme Immunoassay

Enzyme Linked Immunosorbent

Immunoassay

Electron Multiplier

Ephedrone

Florida Highway Patrol

Fluorescence Polarization Immunoassay

Gas Chromatography 
HS-SPME

IA

K-9

KIMS

LC-TMS

LDPE

LLE

LSD

bk-MDEA

MDMA

MDPV

MEPH

MS

NE

NESTT

NMR

NPV

PA

PBSO

PDMS

PDMS/DVB
Headspace Solid Phase Microextraction

Immunoassay

Canine

Kinetic Mirco-Patricle Immunoassay

Liquid Chromatography Tandem Mass

Spectrometry

Low Density Polyethylene

Liquid-Liquid

Lysergic acid diethylamide

3,4-Methylenedioxy-n-Ethylcathine

Methylenedioxymethampehtamine

Methylenedioxyprovalerone

Mephedrone

Mass Spectrometry

Neroprinephine

Non-hazardous Explosive for Security Testing and Training

Nuclear Magnetic Resonance Spectroscopy

Negative Predictive Values

Polyacrylate

Palm Beach Sheriff Office

Polydimethylsiloxane

Polydimethylsiloxane/Divinylbezene 
PEG

PPV

$\alpha-P V P$

RIA

SERT

SOP

SPE

SWGDOG

THC

TLC

T-test

US

VOCs
Polyethylene Glycol

Positive Predictive Values

$\alpha$-Pyrrolidinovalerophenone

Radioimmunoassay

Serotonin Transporter

Standard Operating Procedure

Solid Phase Extraction

Scientific Work Group on Dog and Orthogonal detector Guidelines

Tetrahydrocannabinol

Thin Layer Chromatography

Statistical Significance Test

United States

Volatile Organic Compounds 


\section{Introduction}

In November 2010, Dickie Sanders, a BMX rider, consumed a new drug purchased under the name of Cloud $9^{1,2}$. While hallucinating that twenty-five police cars were outside his home, he took a butcher knife and cut himself ear to ear ${ }^{2}$. Miraculously, by missing major arteries, Sanders was able to receive stitches and recover at home where he continuously told his family "I can't handle what this drug has done to me". Finding it nearly impossible to sleep even after initially taking the drug a day before, Sanders was still experiencing side effects of consumption. While attempting to soothe his son into gaining some much needed rest; Sanders slipped away from his father as he slept. He was able to grab an old 0.22 caliber rifle, place the trigger in his mouth, and take his own life; without anyone ever hearing a sound ${ }^{2}$. Less than a year later the town of Spanaway, Washington was stricken by a similar incident involving a respected army medic.

In April 2011, respected Army Sergeant David Stewart was engaged in a high speed chase with a state trooper, where he pulled out a gun and shot his wife Kristy in the head $^{3}$. After crashing the car, the state trooper approached the vehicle where he witnessed Sgt. Stewart pull out the same gun and end his own life ${ }^{3}$. Home surveillance completed by local officials revealed a house in complete disarray. While surveying the premises police also recovered the body of his five year old son who died of suffocation. Investigations concluded by recovering 500 milligrams of bath salts found in his home, car, and front pocket ${ }^{3,4}$. The death of a promising athlete and a respected family asked questions about the type of potent drug that could result in such dire consequences. 
However while Washington began noticing a new trend in narcotics, it was not until a year later that this new illicit drug captured the attention of the nation.

On May 26, 2012 the world was awakened by the recording showcasing the shocking death of Rudy Eugene. The gruesome scenario showed Eugene, naked, gnawing off 75\% of a homeless man's face under a Miami causeway ${ }^{4}$. Officers who encountered Eugene stated that he was overly strong, appeared to have a demonic look, and failed to respond to several commands probing him to halt his heinous actions ${ }^{4}$. With his unwillingness to respond to law officials demands, Eugene was finally taken down after four shots. With the testimonies from various witnesses and officers, alongside footage, Eugene's incident quickly became famed as the "Walking dead" and "Miami zombie". The assumed cause of such an event was said to be a as result of consuming a new synthetic drug that was quickly taking over the streets. The new drug was called "bath salts".

Encountered numerous times in the field before, officers recalled the behavior of Eugene, citing he showed classic signs of someone on bath salts. However, The MiamiDade Medical Examiner's office only detected components of marijuana in the biological sample tested" ${ }^{5}$ The examiner, Dr. Bruce Hyma, stated that "the laboratory [did] not detect any other street drugs" and thus with the "limits of current technology....marijuana was the only drug that [could be] identified ${ }^{5,6}$." Yet as many questions circulated about how an individual displaying signs consistent with the consumption of bath salts was not detected; Dr. Bruce Goldberger was able to shed some light. A professor and Director of Toxicology at The University of Florida, Dr. Goldberger, stated that the challenge today for toxicology labs is staying on top of new chemicals, new formulations for synthetic 
drugs, and their methodologies; it is very difficult and very expensive ${ }^{5,6}$. From the methodology that the Miami-Dade crime lab possessed, marijuana was the only drug detected, all other synthetic drugs, including bath salts, were not detected as there was no method capable of detecting such drugs; though they may have existed in the biological samples.

Unlike Sanders and Stewart, Eugene's case was among the first where identifying the drug actually consumed relied solely on toxicology reports; without the confirmation or supporting physical evidence to show that Eugene had taken bath salts (unlike in Sander's and Stewart's incident). With many in the nation exposed to the full potential of bath salts, questions began to arise. What are bath salts and how are they created? What are the side effects of taking bath salts? How do we detect and remove these drugs from local communities? In January 2011, the Drug Enforcement Administration (DEA) took a step forward by emergency scheduling baths salts as schedule I drugs, along with 38 other states following suit at the time ${ }^{7}$. However, as seen in the cases above, a vast quantity of these drugs are still reaching consumers.

The statements of Dr. Goldberger's and other officials have shed light on how unprepared law officials and laboratories can be at detecting new synthetic drugs that are released into the states. Therefore the present study examines the chemical makeup of bath salts. The goal of the research study was to develop a method for the extraction of volatile organic compounds (VOCs) from bath salts. An additional goal of the current research study was to isolate a VOC that could be used for the creation of a pseudo training aid in the formation of Controlled Odor Mimic Permeation Systems (COMPS). 
Once completed, this study's COMPS will be used to imprint canines for the detection of bath salts within routine field searches and across the United States Border.

\section{Literature Review}

\subsection{DEA and Illicit Drugs}

Drug use in America is a phenomenon that dates back several decades; from everyday marijuana and opium usage, to more highly profiled substances like cocaine and heroin. Initially used for therapeutic and medical reasons, the use of illicit substances has been a federal concern for the United States for more than a century. Since the 1900s, caution about the overwhelming use of narcotics and dealing with the adverse effects following its consumption became major concerns for federal and law enforcement agencies. During World War I most drugs, such as morphine and cocaine, were specifically used for medical purposes. Most families would send their sons on active duty, small packages of heroin and cocaine to aid in pain relief suffered from various wounds and injuries ${ }^{8,9}$. The first drastic upshift of drug use occurred after the First World War and during the Great Depression. As a coping mechanism for the heightened levels of anxiety, more individuals began consuming and abusing drugs. With widespread drug usage stretching across the United States from the southwest, legislators felt the pressure to implement NEW regulations ${ }^{10}$. During World War II, with the creation of new drugs such as amphetamines and methamphetamines and the major increase in consumption amongst soldiers and civilians; it became critical for the Unites States to establish and maintain an active role in the control and regulation of narcotics. 
The Narcotic Control Act of 1956 set forth a major foundation in the regulation of possession, trafficking, and importation/exportation of all drugs ${ }^{11}$. Under this act, it defined the realms of each qualifying regulation and established the consequential minimum prison sentences for breaking such laws. The control act expressed that it was unlawful for any individual to possess and purchase marijuana, heroin, and cocaine. Outside qualified officials such as doctors, pharmacist, or a licensed individual; the possession of any narcotic was prohibited without the proper documentation. Secondly, the act stated that the transportation of all drugs was unlawful. It forbid an individual to ship, physically transport, or deliver any drug throughout the United Sates and District of Columbia. The importation and exportation of all narcotics were factors also regulated under the act of 1956. Under this control act it stated that anyone who knowingly imports any narcotic into the United States or its territory including receiving, concealing, buying, and selling any imported drug, had committed a violation of this law.

As previously stated, the Narcotic Control Act of 1956 not only structured the legality of drug possession, it also implemented and enforced minimum sentencing for violation of any amendments within this law ${ }^{9}$. Violations of the laws executed under this act were subject to imprisonment by no less than five years to no greater than twenty; with a fine to be no larger than $\$ 20,000$. Second offenses were punishable by imprisonment of no less than ten years to no greater than forty; with a fine no larger than $\$ 20,000$. Whereas importing any drug was equal to a minimal sentence of seven years to life in prison. As a turning point in legislation, The Control Act of 1956 was the first law used to fundamentally set forth punishment for any type of illegal activity stemming from the possession, trafficking, and importation of all illegal narcotics. The act was also the 
first to introduce the death penalty as a result of selling to a minor, if deemed appropriate by a jury panel.

However, even after the creation of the Control Act of 1956, the Unite States still underwent a drastic increase in the number of individuals consuming in drugs nationwide. With reports of more than half a million addicts, government officials were perplexed with how to forcefully manage and take the reins on the overwhelming consumption of drugs ${ }^{12}$. It was not until 1971 that President Richard Nixon declared that the number one public enemy to the United States was drug abuse ${ }^{12,13}$. On July 10, 1973 President Richard Nixon signed executive order No. 1172, for the creation of a task agency under the Department of Justice. This task force would combine all previously established efforts and solely focus on the control and regulations of all narcotics. The Drug Enforcement Administration (DEA) task force was activated in response to the alarming number of individuals who partook in drugs, despite government official's best efforts to hinder its growth.

In efforts to establish a system for control substances, the DEA divided the drugs into schedules ranging from 1 to $5^{14}$; on the basis of set criteria. Substances that were created were submitted to the Control Substance staff to evaluate the drug's potential for abuse and the recommended scheduling for it. The three criteria a substance is examined on are abuse potential, medical use, and dependency. As defined by the Controlled Substances Act, abuse potential refers to any nonmedical use of a drug that is routinely consumed even sporadically for the psychoactive effects it produces ${ }^{15}$. Although certain substances (i.e., cocaine) were initially extracted for medical purposes, testing these 
drugs for proven medical benefits is a necessity. The last criterion for scheduling is dependency on the narcotic once ingested. Physical dependence is observed when an individual experiences withdrawal symptoms (shakes, chills, body aches, nausea, diarrhea) when consumption has not recommenced in a certain time frame ${ }^{16,17}$. In efforts to avoid such symptoms, users will re-administer the drug into their system after an allotted amount of time has passed. Identified as the emotional addition, psychological dependency occurs when the person believes that they need the drug in order to function or perform certain routines in their daily lives. These routines may include going to work, falling asleep, performing well in school, and many more. Also experiencing withdrawal symptoms like crankiness and mood swings, psychological and physical dependency are the last criterion of examination. Once researched, drugs are placed into schedules as depicted in Table 1, where schedule I are considered the most dangerous drugs. 
Table 1.Drug Enforcement Administration's Definition for Narcotic Scheduling and Examples of Each

\begin{tabular}{|c|c|c|}
\hline \multicolumn{3}{|c|}{ DEA Narcotic Schedules } \\
\hline Schedule I & $\begin{array}{l}\text { 1. High abuse potential } \\
\text { 2. No accepted medical use for } \\
\text { treatment in the US } \\
\text { 3. Lack of safety for use under } \\
\text { medical supervision }\end{array}$ & $\begin{array}{c}\text { Heroin } \\
3,4- \\
\text { Methylenedioxymethapetamine } \\
\text { Cathinone (in Khat) }\end{array}$ \\
\hline Schedule II & $\begin{array}{l}\text { 1. High abuse potential } \\
\text { 2. Accepted medical use for treatment } \\
\text { with severe restriction } \\
\text { 3. Abuse may lead to serve } \\
\text { psychological and physical } \\
\text { dependence }\end{array}$ & $\begin{array}{l}\text { Cocaine } \\
\text { Methamphetamine } \\
\text { Opium }\end{array}$ \\
\hline $\begin{array}{l}\text { Schedule } \\
\text { III }\end{array}$ & $\begin{array}{l}\text { 1. Has a lesser abuse potential than } \\
\text { schedule I and II substances } \\
\text { 2. Accepted medical use for treatment } \\
\text { 3. Abuse may result in moderate to } \\
\text { low physical of high psychological } \\
\text { dependence }\end{array}$ & $\begin{array}{l}\text { Anabolic steroids } \\
\text { Ketamine } \\
\text { Thiopental }\end{array}$ \\
\hline $\begin{array}{l}\text { Schedule } \\
\text { IV }\end{array}$ & $\begin{array}{l}\text { 1. Low abuse potential in comparison } \\
\text { to Schedule III } \\
\text { 2. Accepted medical use for treatment } \\
\text { 3. Abuse may lead to limited physical } \\
\text { or psychological dependence } \\
\text { compared to Schedule III }\end{array}$ & $\begin{array}{c}\text { Alprazolam (Xanax) } \\
\text { Clonazepam } \\
\text { Cathine (in Khat) }\end{array}$ \\
\hline Schedule V & $\begin{array}{l}\text { 1. Low abuse potential compared to } \\
\text { Schedule IV } \\
\text { 2. Accepted medical use for treatment } \\
\text { 3. Abuse may lead to limited physical } \\
\text { or psychological dependence } \\
\text { compared to Schedule IV }\end{array}$ & $\begin{array}{c}\text { Pyrovalerone } \\
\text { Pregabalin (Lyrica) } \\
\text { Ezogabine }\end{array}$ \\
\hline
\end{tabular}

Since the implantation of the DEA task force there has been a considerable number of law enforcement raids conducted to disrupt large operations of drug smuggling, distribution, and selling within the United States. A large percentage of raids have successfully seized drugs such as heroin, cocaine, marijuana (schedule I), 3,4Methylenedioxymethapetamine (MDMA), Methamphetamine (MA), steroids, and large bundles of what is considered as "drug money"(currency exchanged during the sale of 
narcotics). During the beginning years of this task force most of the narcotics encountered were among those previously stated. As newer drugs emerged, new scheduling and guidelines were implemented and enforced. Of those newer substances, two components scheduled in different categories were derived from the same origin known as khat. It was from this plant's composition that a major evolution into a deadly narcotic would arise and shift the standard means of "war on drugs".

\subsection{Khat}

Khat is a perennial green tree or large shrub grown in the Horn of Africa and Arabian Peninsula ${ }^{18}$. Also referred to as qat, kat, chat, miraa, Catha, and Africa tea, the meaning of khat is derived from the Latina word Catha edulis (C. edulis) of the

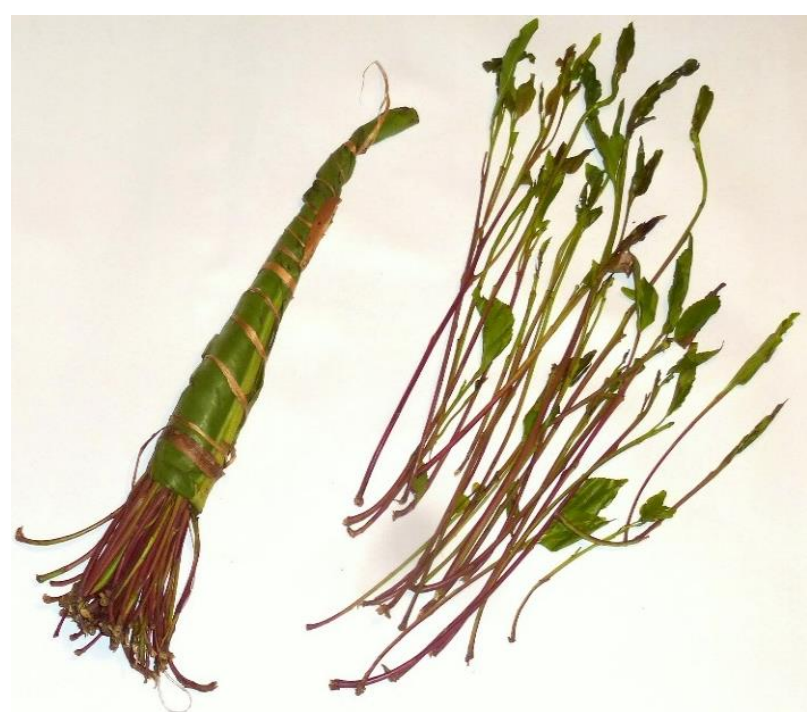

Figure 1. Khat plant ${ }^{1}$
Celastraceae family. Growing at altitudes higher than 1500 meters, the khat plant is six- seven meters in height with a slender thin trunk appearing to have a greyish-brown coloration. With leaves two- five centimeters wide and five- ten centimeters long, the leaves of a khat are described as possessing a leathery brownish-green appearance

that harden and become bleached as they mature ${ }^{19}$. With a leaf odorant that's faintly aromatic and a slightly sweet and astringent taste, the khat plant is known for the psychoactive effects experienced after its consumption ${ }^{19,20}$. 
Traditionally the khat plant was used as part of a chewing ritual in Wallo, Africa. Religiously, Muslim participants gathered for a wadaja ritual in efforts to send good words to the heavens for blessings and to come together for an individual's sickness ${ }^{21}$. Mostly comprised of men; the host would gather at his house and pass out the cuttings of a course leaf (known as jafjaf) from a khat tree and chew on them. If after the first round the euphoric effects were not felt, the men would rub their hands together in a request for more. Once the stimulant effects were experienced the men would take turns offering up words of encouragement and blessings to the skies for good fortune. The khat chewing ritual would last for hours or until the supply ran dry. By chewing the plant the participants were able to suppress their hungry and stay awake for extended periods of time; allowing for prolonged periods of worship ${ }^{21}$. Before the 1980s, when khat began selling overseas, the practice of khat chewing was not frowned upon; as it only served to aid in the religionist needs of the Wallo Muslims ${ }^{22}$. During the nineteenth and twenty century the marketing and cultivation of khat increased. After the cold war the marketing for khat became competitive as more western settlers desired the stimulant effects provided. The wildly grown jafjaf plant was soon replaced with smaller tenderer khat trees, that were farmed and organized ${ }^{22}$. The leaves were cut, bundled, rolled in leaves, and placed in plastic bags as a more marketable appeal, similar to Figure $1^{23}$. Initially the short span of euphoric effects was the primary reason for the khat's confinement to Africa. After cutting, the stimulant effects could only be experienced within the day. However, with the evolution of expressed shipping, it became much more feasible to deliver the khat twigs faster and to countries further away. After the wide spread 
occurrence of khat chewing outside the Wallo tribe, many critics became concerned with the use and overall consumption of khat for no reason other than its psychoactive effects.

Stimulants are any substances that cause an excitation within the bodily functions more specifically the Central Nervous System $(\mathrm{CNS})^{20,24}$. As a stimulant, the effects from consuming khat include an increase in alertness, overall happiness, and talkativeness (all symptoms expressed by the Muslims of Wallo as useful for rituals). Traditionally there are five stages of intoxication that are experienced with the consumption of khat ${ }^{23}$. The first is the cheerful excited stage which can last from one to two hours, and is highly favored among young chewers. The second stage brings slight irritability and emotional awareness of problems followed by transition into the dark stage. Sensitivity to light begins in the third stage, where most chewers prefer to sit in the dark even after the sun has set. The imagination becomes overly active, creating vivid intense feelings of excitement. The fourth stage is the depressive phase, where personal problems and selfconvictions begin to plague the chewer's mind. The responsible factor for these feelings is the dwindling effects of the khat initially chewed; most users begin to spit the remains out and chew more to bypass this phase. Lastly, anorexia and insomnia carry on throughout the night, resulting in a lethargic state the next morning. Amphetamine-like effects produced from khat chewing also include suppressed appetite, hyperactivity, elevated blood pressure, dilated pupils, heightened respiration, and lack of fatigue; which are said to last as long as 3 hours ${ }^{20,25}$. Although khat is not a controlled substance the two components responsible for its stimulant effects are classified as schedule I and IV narcotics. 
As a whole, there are several components that have been separated and examined from $C$. edulis; $7-14 \%$ of the weight is contributed by sterols, tanins, alkaloids, and flavonoids ${ }^{23,26}$. The plant chemistry includes a host of amino acids, trace levels of vitamins and toxic metals like lead ${ }^{26}$. The three main alkaloids found in khat<smiles>C[C@H](N)C(=O)c1ccccc1</smiles>

(-)-S-cathinone

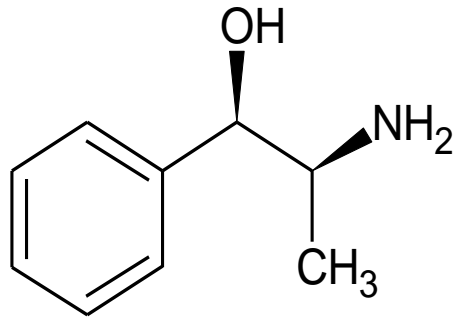

(-)1R,2S-norephedrine<smiles>C[C@H](N)[C@H](O)c1ccccc1</smiles>

(+)-1S,2S-norpseudoephedrine (cathine)

Figure 2. The Active Components of the Khat Plant: Cathinone, Cathine, and Norephedrine

leaves are S- $\alpha$-aminopropiophenene (cathinone), norpseudoephedrine (cathine), and norephedrine (figure 2$)^{23}$. Although these compounds contribute to the stimulant effects of khat, cathinone is the major constituent. With cathine being 10 times less potent, the drug concentration of cathinone found in the khat plant directly correlates to the potency of the plant when chewed. As cathinone is the determining factor of stimulant effectiveness, therein lays the explanation to the short consuming window for maximum 
yield. In room temperature cathinone becomes relatively unstable, decomposing into $(+)-$ norpseudoephedrine and norephedrine completely within 48 hours. This decomposition explains why the leaves are typically chewed within a day of cutting to achieve the greatest stimulant effect. In order to prolong cathinone's breakdown the leaves and shoots are typical wrapped in plastic foil, shawls, or banana leaves, and immersed in water for an allotted amount of time ${ }^{21,23}$. With approximately 100-500 grams of khat being chewed in a session there is an estimated $78-343 \mathrm{mg}$ of cathinone found per 100 grams of freshly cut leaves ${ }^{27}$. Once ingested, mainly through the oral mucosa, cathinone breaks down into cathine and norephedrine, where less than $5 \%$ of the natural cathinone is detected afterward $^{25}$.

Often identified as the natural amphetamine, cathinone is the stimulant produced within khat responsible for the lack of depression and lack-luster state of minds. With years of knowledge pertaining to the side effects experienced from khat, scientist and pharmacist alike began looking into synthetically creating larger quantities of cathinone (compared to the amounts found naturally) for various measures including medical purposes (antidepressant and anorectic agents).

\subsection{Bath Salts and its History}

\subsubsection{History}

For decades opium, psilocybin mushrooms, marijuana, cathinone, and to some extent cocaine are plant derived drugs, as they were derived directly from a plant ${ }^{28}$. The methods of consuming these drugs for psychoactive effects include smoking or eating the plant directly. Even the active ingredient, cocaine, is derived from coca leaves, 
undergoing extractions without major change to the natural molecular structure of the compound. For years it was considered impossible to manufacture plant-derived drugs in a laboratory setting, until that hypothesis was falsified in the 1800s. Friedrich Wohler, a German chemist, created a constituent in human urine named urea in $1828^{29}$. The new area of organic chemistry introduced the creation of organic chemical structures built within a lab using fundamental building blocks. The evolution from vegetable to chemical brought out the term commonly known today as synthetic drugs. Synthetic drugs are defined as substances manufactured from chemicals, often being similar to the natural drug they mimic ${ }^{28}$. Illicit substances that have been manufactured and abused include heroin, methamphetamine, and LSD (lysergic acid diethylamide). In 1928 cathinone, a naturally occurring stimulant from khat was first synthetically modified to produce the first class of synthetic cathinones.

Structurally modified cathinones were initially synthesized for clinical use as antidepressants and anorectic agents (appetite suppressant). In 1928 the first synthetic cathinone created was a purified $\alpha$-methylaminopropiophenone compound known as methcathinone or ephedrone (EPH); figure $3^{27}$. A year later, Saem de Burnaga Sanchez synthesized 4-methylmethcathinone or mephedrone (MEPH). Patented and pharmaceutically manufactured by a US company Parke Davis, EPH and MEPH were ideally produced as CNS stimulants for therapeutic purposes along with cathinone derivatives known as $\mathrm{N}, \mathrm{N}$-diethylcathinone and $\mathrm{N}, \mathrm{N}$-dimethylcathinone (diethylpropion and dimethylpropion respectively) ${ }^{27}$. 


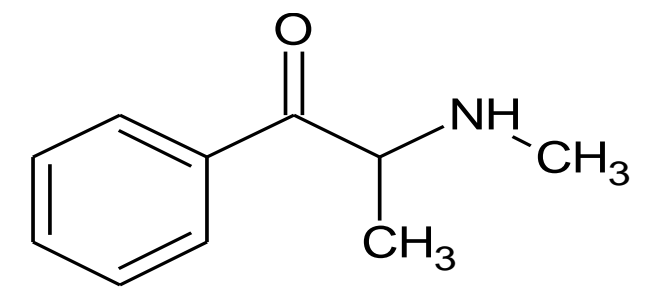

Methcathinone (Ephedrone)<smiles>CNC(C)C(=O)c1ccc(C)cc1</smiles>

4-methylmeth-cathinone (Mephedrone)

Figure 3. The Molecular Structure of the First Synthesized Cathinones, Ephedrone and Mephedrone

Initial observation after consumption not only witnessed desired effects of euphoria and empathy, but also greatly decreased an individual's sense of insecurity while increasing their libido ${ }^{30}$. However, a significant problem was the potency of these drugs. Within a short time frame of release, the first cathinone derivatives began displaying severe addictive potential more potent than both cocaine and cathinone ${ }^{31}$. In efforts to curb their addictive characteristics, scientist began developing other synthetic cathinones. In the 1970s 4-methyl- $\alpha$-pyrrolidinopentanophenone, pyrovalerone, was synthesized to treat fatigue, obesity, and lethargy ${ }^{32}$. Although useful, it caused addition and was thus pulled from all clinical treatment programs. Created in 1996, methylone was synthesized and patented under the idea of being used as an antidepressant and antiParkinsonism agent ${ }^{33}$. Nonetheless, for the same reason this drug was never manufactured for any purposes, scientifically, beyond what was drafted within the patent. Unfortunately with the high yield of addictiveness associated with synthetic cathinones primarily developed from the late 1920s to the late 1990s, most synthetic cathinones 
developed never were optimally used for any therapeutic purpose and were eventually shelved.

In the mid-2000s methylone emerged as one of the first synthetic cathinones sold for the sole purpose of recreational use, under the name "Explosion" (in the Netherlands and Japan) ${ }^{27}$. Along with methylone the drug known as M-Cat, TopCat, and miaowmiaow (previously known as Mephedrone) also began emerging as a legal alternative to cocaine. Around 2008 it was reported that in addition to methylone and mephedrone the recreational sell of synthetic cathinones also included ethcathinone, flephedrone $(\mathrm{N}-$ ethylcathinone), butylone, ethylone, and $\mathrm{MDPV}^{27,30}$. With the overall psychoactive effects similar to cocaine and more potent than the amphetamines, this first wave of synthetic cathinones gained vast popularity as a legal alternative to the strict regulation on more "traditional" stimulants. Within the US, the influx of synthetic cathinones coming into the country was not reported until 2010 when the American Association of Poison Control experienced a drastic increased in the number of calls pertaining to a new trend of drugs; referred to as bath salts (synthetic cathinones, figure 4$)^{34,35}$. Yet this trend declined only after consumers became increasingly more knowledgeable about the side effects. Marketed throughout the internet and headshops, synthetic cathinones were easy to purchase as cheap alternatives to cocaine, meth, and MDMA. 


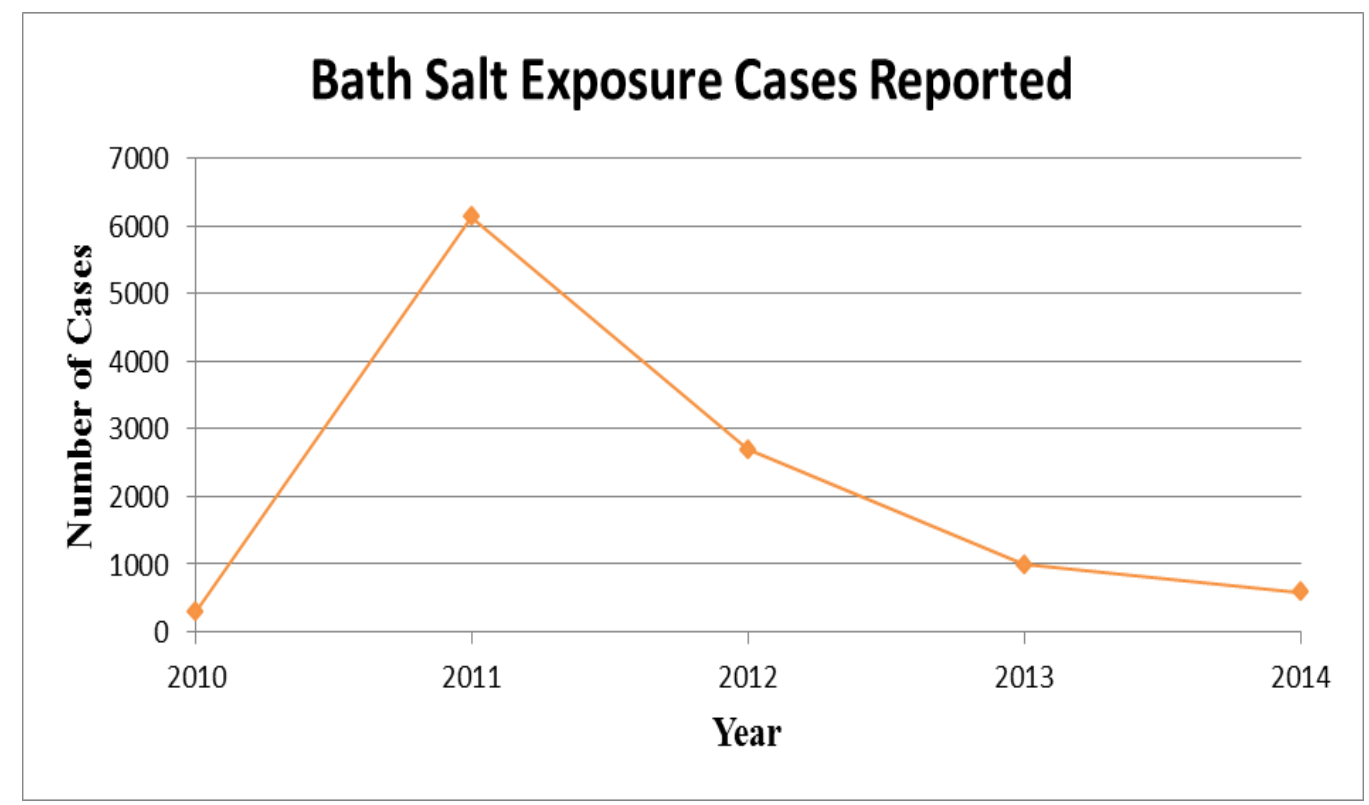

Figure 4. The Annual Reporting of Cases Called into the American Association of Poison Center with Questions in Regards to the Exposure of Synthetic Cathinones (Bath Salts) ${ }^{34}$

With no legal ban of synthetic cathinones they were routinely sold at various headshops, smart shops, gas stations, contained in small bags for personal consumption; or in bulk supply online. Often sold in quantities ranging from 50mg to $10 \mathrm{~g}$ bags under a variety of names (Vanilla Sky, Ivory Wave, Meow Meow, Blue Silk, etc.), users quickly became familiar with the drug labeled "not for human consumption" or "research chemical" accompanied by a smiley face ${ }^{27,36,37}$. Synthetic cathinones are routinely sold in various forms. Typically these drugs appear to be a white, tan, or yellowish crystalline or amorphous powder stored in a bag or capsules ${ }^{27,38}$. Yet, various street forms also include dark brown, orange, and faint green coloration of the crystals, figure 5.

The most common methods for consuming synthetic cathinones are through oral ingestion and insufflation. Users typically snort the drug or engage in an activity called 
"keying", where a key is dipped in the powder and then insufflated ${ }^{39}$. The oral ingestion of synthetic cathinones occur through the routine manner of swallowing a capsule or by wrapping the powder in cigarette paper and smoking it (a technique called bombing) ${ }^{27,30}$. Alternative methods also include rectal insertion and intravenous/intramuscular injection although less common ${ }^{40}$.
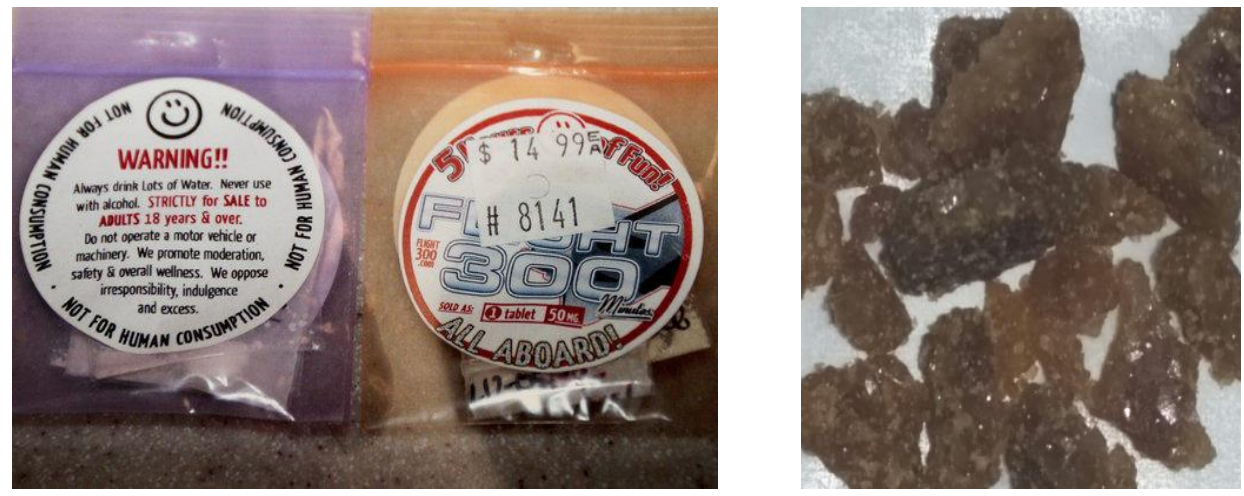

\section{Figure 5. The Packaging of Bath Salts Sold in Headshops (Left $)^{41}$ and the Appearance of the Drug which May be Housed in These Packages (Right)}

\subsubsection{Pharmacodynamics}

The potency and euphoric effect resulting from synthetic cathinones associated with their effects on the nervous system. Neurochemistry defines the mechanism of the communication within the central nervous system (CNS). As depictured by figure 6(A), communication between two neurons typically begins with an action potential traveling along the axon of the presynaptic neuron. This impulse causes vesicles, carrying neurotransmitters (chemicals), to transition down to the membrane of the neuron, fuse together and release the transmitters into the open space.

The open space between two neurons is called the synaptic cleft. The post-synaptic neuron's membrane is lined with receptors that bind with chemicals as they drift across 
the synaptic space. The binding triggers an action potential in the post synaptic neuron which then passes the message along. Afterwards the transmitters are released back into the synaptic space. Here, they are either broken down after interacting with an enzyme or migrate back into a presynaptic neuron via a neurotransmitter transporter. There are several chemical substances which travel through the chemical system to keep the CNS functioning. Three important chemicals which are influenced by the intake of drugs, and subsequently the reason behind using synthetic cathinone are the monoamine substances. Synthetic cathinone interfere with the reuptake or release dopamine, noradrenaline, and serotonin (monoamine chemicals) ${ }^{30}$.

Each neurotransmitter is responsible for its own function and has a role it plays in the human CNS. Dopamine is responsible for the feeling of pleasure, reward motivated behavior, attention, and memory ${ }^{42}$. Noradrenaline (also known as norepinephrine; NE) is the transmitter mostly responsible for the "fight-or flight" response that all animals with backbones experience ${ }^{43}$. Its role is to create alertness, arousal, vigilance, and increase memory when the brain and situation deem it necessary. The last transmitter discussed, serotonin, is responsible for the mood or feeling of happiness experienced by an individual $^{43}$. Serotonin functions to affect a human's sexual desire, sleep, body temperature regulation, and appetite. 

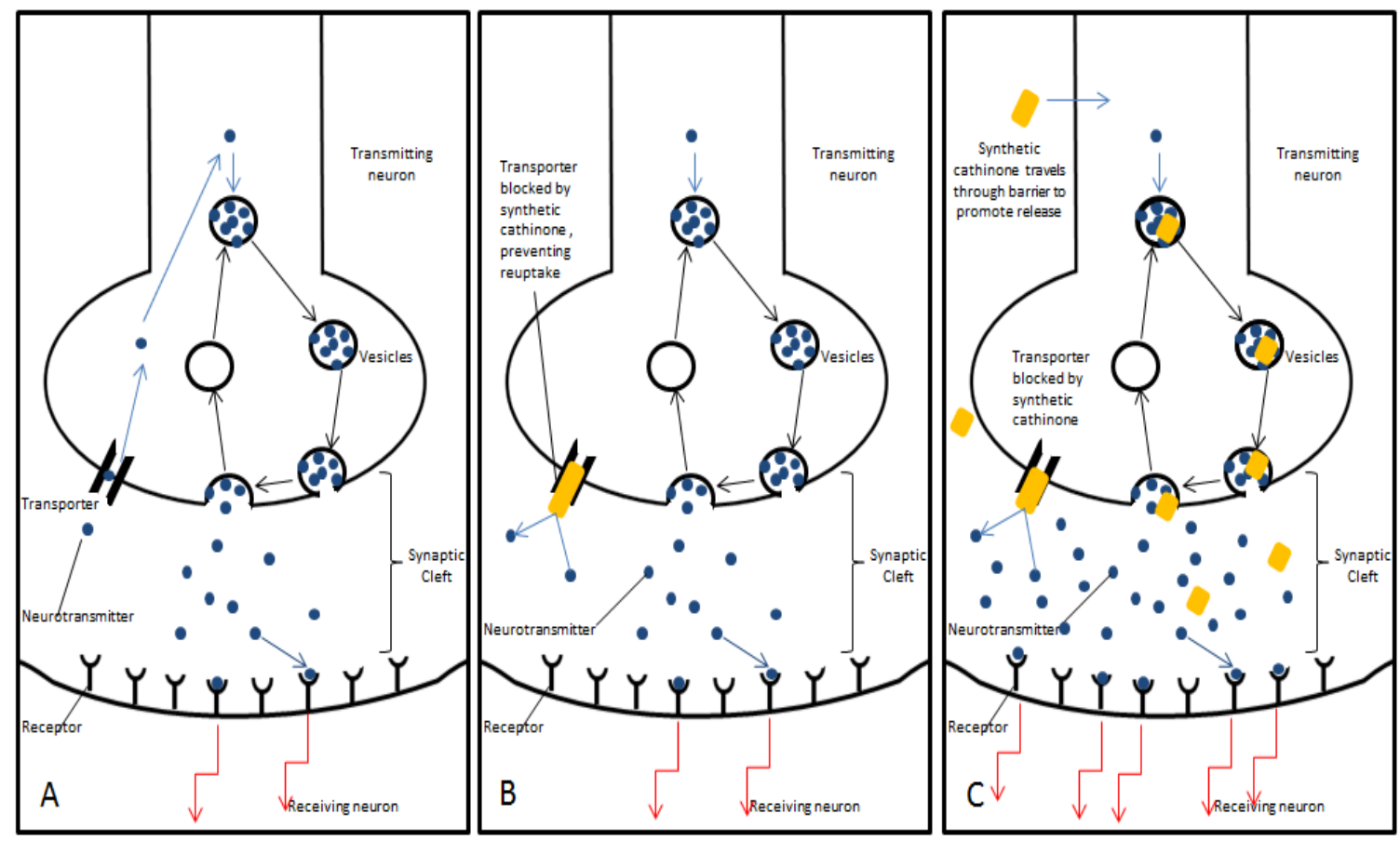

Figure 6. The Phases of Neurochemistry: The First Phase is where the Neurons communicate regularly through the Promotion of the Action Potential (A). The Second Phase the Drug of Question Blocks the Transporter Permitting the Reuptake of Certain Monoamines (B) The Third Phase the Drug Blocks the Uptake and Releases More Monoamines $(C)$.

When synthetic cathinones are consumed they are believed to act as reuptake inhibitors and promote the release of certain monoamine transmitters ${ }^{30,39,44}$. Synthetic cathinones similar to EPH and MEPH release dopamine (DA) through DAT (dopamine transporter) and release serotonin (5-HT) through the SERT (serotonin transporter). The potency in which they release DA is lower than MDMA, and high concentrations of these drugs are required in order to achieve potent amounts of serotonin release concentrations $^{37}$. Later cathinone derivatives such as provalerone, naphyrone, and MDPV (Methylenedioxyprovalerone) are similar to cocaine, in which they act as potent transport 
inhibitors blocking the reuptake of dopamine and serotonin, figure $6(\mathrm{~B})^{30,39,45,46}$. Synthetic cathinones, like methcathinone, have also been known to initiate pharmacodynamics similar to amphetamines ${ }^{46}$. These drugs are able to permeate through the neuron barrier and initiate the release of more monoamines without the need of any action potential, figure $6(\mathrm{C})^{30,39}$. Consumption of chemically modified cathinones were believed to be beneficial as anti-depressants, for the increased amount of neurotransmitters they cause. Within the synaptic cleft, a greater number of these chemicals allowed for a larger number to bind with receptors of the receiving neuron, therefore sending a stronger feeling of reward. Increased amounts of DA, 5-HT, and NE were deemed counteractive as they gave an individual pleasure and happiness which under a depressed state are much lower. Synthetic cathinones, as previously mentioned were also created in hopes of combating obesity. Known as an appetite suppressant, consuming synthetic cathinones allowed for the release of more 5-HT transmitters in the cleft allowing for a prolong feeling of fullness, thus curving the appetite of a large individual; resulting in weight lost $\mathrm{t}^{27,44,45}$.

\subsubsection{Pharmacokinetics}

Following the intake of synthetic cathinones, these derivatives immediately undergo extensive phase I metabolism. The exact pathway taken differs depending on the molecular structure of the cathinone derivative. The first stage is the reduction of the $\beta$ keto moiety into an alcohol followed by the demethylation of the methyl group (cathinones similar to methcathinone and mephedrone). Derivatives structurally similar to butylone, methylone, and ethylone undergo metabolism by demethylation followed by the keto reduction, $\mathrm{N}$-dealkylation, and o-methylation. The metabolizing of cathinones 
containing pyrrolidino groups take place by transforming the $\beta$-keto position on the ring to an alcohol before undergoing biotransformation to procedure a lactam or a primary amine. Synthetic cathinones appear mostly as metabolites in the urine with least than $10 \%$ of the unmetabolized derivative being detected in the sample.

Because synthetic cathinones are less lipophilic than amphetamines and methamphetamines, the dosage amount pertaining to each type tends to be higher in order to achieve similar or greater effects. Mephedrone has a reported dosage of 15 to $250 \mathrm{mg}$ for oral ingestion and 5-125 mg for insufflation with psychoactive effects occurring between 10-20 minutes after consumption and lasting for 2-4 hours ${ }^{27,30}$. Methylone has a reported oral dosage ranging from 100-300 $\mathrm{mg}$ (with amounts larger than $250 \mathrm{mg}$ considered to be a heavy dosage) with effects beginning in 15-30 minutes and lasting over 3 hours ${ }^{47,48}$. With consuming methylone it has also been expressed that most of the effects take place over the next 24 hours after the drugs have been consumed, during the “down" process. The little research conducted on Butylone suggests a window of 4-6 hours. 3,4-Methylenedioxypyrovalerone (MDPV), potency often expressed as stronger than cocaine, has a dose amount of 5-20 mg. Although users have experience very unpleasant effects when consuming amounts larger than $15 \mathrm{mg}$, some users ingest quantities as high as $200 \mathrm{mg}^{27,38,39,48}$. 


\subsubsection{Cases and Toxicity}

Synthetic cathinone or bath salt intoxication has been heavily experienced due to over consumption. Oftentimes patients will increase their intake for an increased feeling or longer duration of euphoric. Others will overlay doses in effort to stop the adverse effects of coming off the drugs, during the down phase. When an individual consumes quantities outside the typical range for bath salts they experience increased desired physcostimulatnt effects including unwanted ones such as paranoia, hallucinations, excessive agitation, anxiety, talkativeness, time lost, sweating, vomiting, muscle twitch, suicidal thoughts, tachycardia, vertigo, Parkinson, and many more ${ }^{37,40,44,49}$. The length of time which these effects last can range from hours to months with the appropriate medical care, while some cases end in death, table 2.

\section{Table 2. Case Reports of Toxicity from Synthetic Cathinone Use}

\begin{tabular}{|cccc|}
\hline Year & User & Substance & Outcome \\
\hline $\mathbf{2 0 1 0}$ & Male/31 & Bath salts & $\begin{array}{c}\text { Renal failure/rhabdomyolysis } \\
\text { resolved over 3 days }\end{array}$ \\
\hline $\mathbf{2 0 1 1}$ & Male/20s & $\begin{array}{c}\text { Bath salts } \\
\text { (MDPV } \\
\text { detected) }\end{array}$ & $\begin{array}{c}\text { Mental status normalized } \\
\text { after 30 hours from intake }\end{array}$ \\
\hline $\mathbf{2 0 1 2}$ & Female/24 & $\begin{array}{c}\text { Methlyone, } \\
\text { butylone }\end{array}$ & $\begin{array}{c}\text { Renal failure followed by } \\
\text { death }^{52}\end{array}$ \\
\hline $\mathbf{2 0 1 3}$ & Male/19 & $\begin{array}{c}\text { Bath salts } \\
\text { (methylone } \\
\text { detected) }\end{array}$ & Collapse and death $^{53}$ \\
\hline & & & \\
\hline
\end{tabular}




\subsubsection{Laws and Regulation}

Commercially, synthetic cathinones are sold with containers labeled "not for human consumption", "plant food", or "plant fertilizer" directly within headshops and gas stations. While readily easy to obtain locally and via the internet, the popular trend of consuming bath salts quickly grew within the United States. By simply googling the name of the drug desired, a vast number of websites are generated catering to the "retail" sale of bath salts at discounted prices. On September $8^{\text {th }} 2011$, in an effort to combat the drastic increase of cases pertaining to bath salt overdosing; the DEA issued a notice of intent to temporarily schedule three synthetic cathinones (mephedrone, methylone, and MDPV) under the Controlled Substance Act (CSA) $)^{7,54}$. The notice was issued as a respond to the "imminent hazard to the public's safety" in regards to the listed drugs. As DEA's emergency schedule banned the possession and consumption of the previously listed drugs, amateur chemist found a way to avoid such regulations.

The second generation of recreational synthetic cathinones were released under

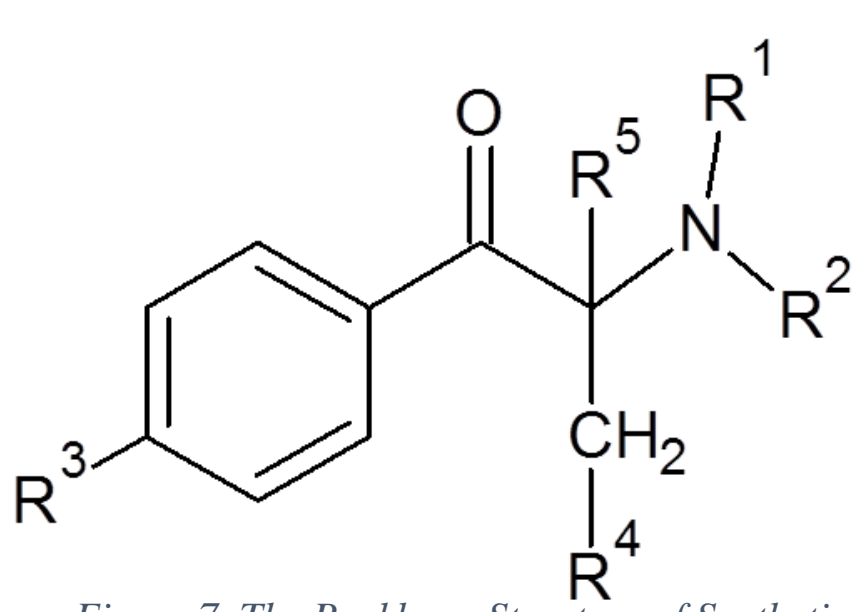

Figure 7. The Backbone Structure of Synthetic Cathinones. The $R$ groups are Active Sites where the Molecule can be built on to Create New Derivatives of the Drug the names flephedrone, butylone, ethylone (bk-MDEA), 3-

fluoromethcathinone, and naphthylpyrovalerone (naphyrone); in efforts of avoid regulations. Shortly after these drugs the third generation of synthetic cathinones 3,4-DMMC (3,4dimethylmethcathinones), pentedrone, 
and $\alpha$-PVP ( $\alpha$-pyrrolidinovalerophenone) began appearing. Chemically modified cathinones were structurally similar to first generation derivatives only deviating by the addition of a molecular component to the back bone of the cathinone structure, figure 7 . The term "legal highs" was quickly coined to the second and third generation as they did not fall under the scheduling laws implemented and were thus legal to possess. By avoiding laws through loopholes, users were under the impression that these drugs were safe to consume, once again spiking an increase in the toxicity cases increased. To close these loopholes the DEA placed another emergency scheduling on ten additional cathinone derivatives on January 28, 2014. To date more than 30 different types of synthetic cathinones have been documented within the US and Europe $\mathrm{e}^{27,38}$.

As laws regulating the possession of synthetic cathinones are more defined to cutoff the potential for loopholes, a problem still remains that a large influx of these drugs are still pouring into the states within reach of dealers and consumers. Current methods of detection for routine drugs such as cocaine, methamphetamine, LSD, marijuana, and heroin, have proven to be somewhat challenging for synthetic cathinones.

\subsection{Non-Biological Detection of Narcotics}

\subsubsection{Color/Spot Testing}

Narcotic detection routinely begins with the identification of various physical attributes of any suspected substance including coloration, structure, and consistency compared to what is known from literature. Visual screening of drugs such as marijuana, MDMA, and other tablet forms require very little investigation as these substances are only witnessed in one recognizable form (i.e., green leafy configuration and particular 
color) and/or bear indentations which can easily be search or memorized for general identification. However human observation can often lead to inaccuracies in presumptive testing as similar drugs such as heroin, cocaine, methamphetamine, and various synthetic cathinones are visually similar in their color and consistency. With visual similarities hindering the positive identification of groups of drugs, there became a necessity for a more concrete method of initially identifying the suspected drug encountered.

Presumptive testing, in the forensic science community, is defined as the preliminary analysis of a substance that determines whether an unknown sample is likely to be a substance or is definitely not the suspected substance ${ }^{55}$. The easiest and most routine type of presumptive testing is simply known as color testing. The process involves introducing a small quantity of the substance to a chemical or a mixture of chemicals. The encounter between the chemical(s) and substance initiates a reaction that results in a physical characterization (color change) ${ }^{56}$. The resulting color, table 3 , typically depends on the drug required to initiate such a reaction.

Table 3. Final Colors Produced when Chemical Reagents React with Various Drugs. *involved three separate stages of reaction producing three separate color results ${ }^{56}$

\begin{tabular}{|c|c|c|}
\hline \multicolumn{1}{|c}{ Reagent } & Substance & Color \\
\cline { 2 - 3 } & Cocaine & Strong greenish blue \\
\cline { 2 - 3 } Cobalt Thiocyanate & $\alpha$-PVP & Strong greenish blue \\
\hline Marquis & Methadone & Brilliant greenish blue \\
\cline { 2 - 3 } & Methamphetamine & Deep reddish orange \\
\hline Mecke & Ethylone & Dark reddish orange \\
\hline \multirow{2}{*}{ Duquenois-Levine } & LSD & Greenish black \\
& THC* & $\begin{array}{l}\text { 1. Gray purplish blue } \\
\text { 2. Light purplish blue }\end{array}$ \\
\hline
\end{tabular}


The major problem with color testing is cross reactivity and undistinguishable results. For example, once cocaine reacts with the cobalt thiocyanate reagent the resulting color as described above is a greenish blue. However, synthetic cathinones structurally similar to $\alpha$-PVP also give the same indication color of blue ${ }^{57}$. Although many scientists have come to visually note their difference in consistency, many criminalists mistakenly assume the unknown substance is the wrong drug; hence color testing being categorized as a presumptive test only.

A second type of presumptive testing used in the forensic community is Thin Layer Chromatography (TLC). Operating on the separation of non-volatile mixtures TLC employs a thin material layer of absorbent ${ }^{58}$. Typically the most commonly used types of absorbent are a silica-gel, cellulose, or alumina coated on a thin film, glass, or small piece of metal ${ }^{58}$. Taking the unknown substance and various suspected drug standards each are carefully plotted onto the plate using capillary spotters. After all knowns and unknowns have been plotted the plate is placed in a solvent within a closed container for development. The mobile phase (solvent) will travel up the stationary phase carrying the standards and separating the components of the sample as it travels. Once developed the retardation factor is calculated and compared between the unknown and reference material to suggest what type of narcotic the sample is believed to contain ${ }^{59}$. Although not routinely used within a forensic crime lab, the TLC technique offers a unique approach to presumptive testing. 


\subsubsection{Immunoassay}

Perhaps the most widely used method for preliminary detection of illicit substances is immunoassays. Dr. Solomon Berson and Dr. Rosalyn Yalow, in the late 1950s, first investigated the use of immunoassays in regards to insulin metabolism in patients ${ }^{59}$. Immunoassay (IA) is a technique that employs antibodies to bind with target analyte (antigen) potentially in the substance for identification ${ }^{60}$. Antibodies, also known as immuogluobins, are y-shaped proteins used for their stability, versatility, and specificity ${ }^{60,61}$. Within the assay the analyte concentration is determined quantitatively by measuring the distribution between bond and free forms as the analyte and specific antibody interact.

The basic working principle for immunoassay is generally a system carrying antibodies whether fixed or not and introduction of a sample. If the target antigen is present within the substance a binding reaction will occur at each binding site.

Simultaneously labeled antigens will be introduced to interact with the antibodies in the event the targeted antigen is not present within the sample ${ }^{59}$. The end results are tabulated by various methods showcasing the presence or absence of the target analyte as well as the concentration. Typically immunoassays are classified under two realms competitive or noncompetitive and heterogeneous or homogeneous ${ }^{62}$. Competitive assays are when both the analyte and labeled antigen compete to bind with the antibody. Noncompetitive assays use an excess number of antibodies absorbed in a stationary phase. The sample's analyte then interacts with the stationary phase containing the antibodies to bind with a large percent of the antigen found in the substance ${ }^{59}$. Homo and hetero classified 
immunoassays describe the steps required after the binding reaction has occurred in order to measure the end product and interpret the results. After the binding has taken place, assays that require the separation of the bound and free antigens before detection can occur is known as heterogeneous ${ }^{59,60,62}$. These forms are usually separated through solid phase adsorption liquid extraction, etc. Homogenous does not require the separation to occur as the labels are changed during the binding process making it possible to identify without the need to remove the free labels prior to analysis.

The first type of immunoassay developed was Radioimmunoassay (RIA). This heterogeneous assay uses a fixed known concentration of a radioscopically labelled analyte, known as a tracer, to compete for the binding sites ${ }^{60}$. Following incubation, the bound components are separated through a precipitation process or decanting the solution and the radioactivity is detected by a scintillation counter ${ }^{62}$. Hazardous concerns and the management of radioactive disposal have pushed this type of immunoassay to be replaced by faster, more environmentally safe methods. Enzyme immunoassays (EIA) are replacement methods that involve the use of enzymes to detect the amount of antigen in a sample. Enzyme Linked Immunoadsorbent (ELISA) is readily used in labs as a variant of EIA. Unlike EIA, ELISA is a heterogeneous assay which binds its antibodies to an immobilized wall known as the microtiter plate ${ }^{63}$. The sample is introduced to the well, incubated, and washed to remove all unbound substances. The enzyme conjugated antibodies are then added to the plate wells. A second predetermined incubation period allows the enzyme conjugates to bind to any remaining unbound primary antibodies. After incubation the plate goes through several washing phases to remove any unbound labeled antigen. To end the reaction a substrate is added to the well to bind with the 
enzyme present and produce a color change indicating the end of the reaction ${ }^{64}$. Strong enzyme to substrate binding will visually result in a bright color change. The plates are transferred to spectrometer where the absorbance or fluorescence detected is inversely proportional to the sample's drug concentration, figure $8^{60}$. Although the previous is known as Competitive ELISA there are other commercial available types including sandwich, direct, and indirect.

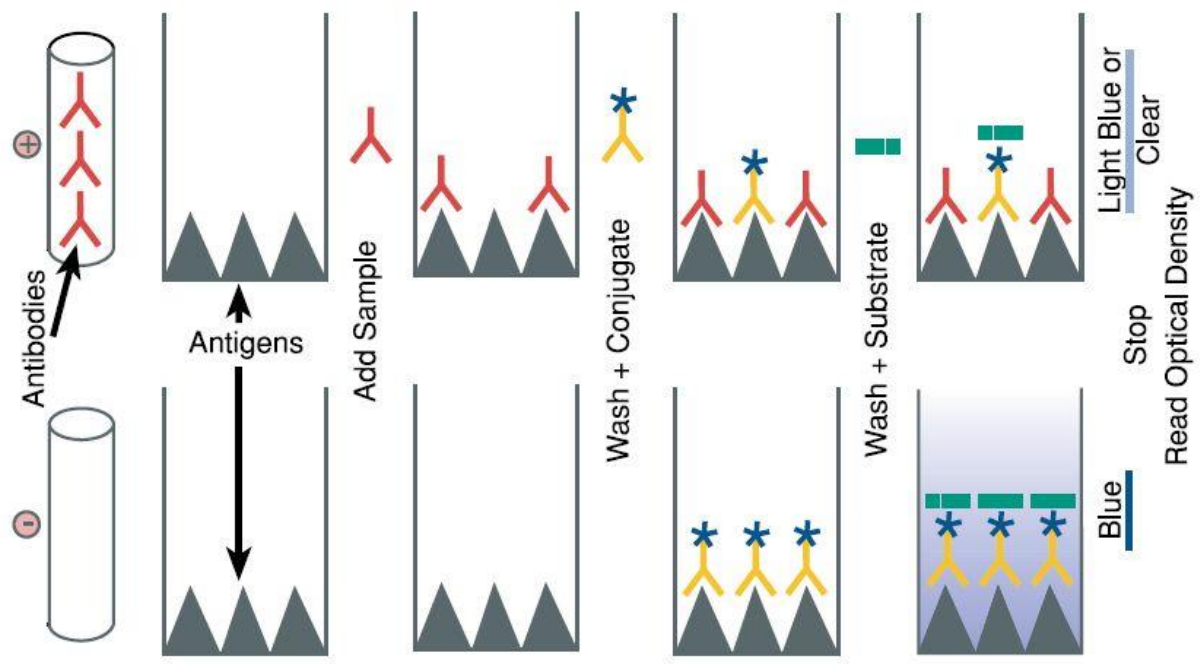

Figure 8. Competitive ELISA where the Bound Antigen or Primary Antibody are Introduced to each Followed by Incubation and Wash. Afterwards an Enzyme Conjugate is added, Incubation and the Washing Phase are Repeated Before a Substrate in Introduced to Bind with the Enzyme Present to Induce a Color Change ${ }^{64}$

An advantage to ELISA, unlike RIA, is that there is no need for radioisotopes to determine drug concentration. Enzyme Linked Immunoadsorbent also achieves a higher specificity and selectivity by using an enzyme, which requires no sample pre-treatment, and only needs a small amount of the specimen ${ }^{59}$. However the sensitivity is only as good as the spectrometer used during the reading process, and the incubation time can be 
extensive (various hours). Alternate immunoassays such as Fluorescence Polarization immunoassay (FIPA), Kinetic Micro Particle Immunoassay (KIMS), Lateral Flow Immunoassay have all been created as variations for drug detection with advantages over ELISA and RIA; in addition to other limitations. Methodology limitations to immunoassays include interferences between the antigens and binding source which hinder the results obtained. Unrelated compounds structurally similar to the target antigen can interfere with the system by interacting with the antibodies changing their effects and causing an increase or decrease in the results. Unlike color testing, TLC, and IA drug detection has also been achieved through biological specimens.

\subsection{Biological Detection of Narcotics}

\subsubsection{Canine Detection History}

Substance detection through biological measures employs the use of any living specimen. Throughout history animals including honeybees, birds, elephants, and dogs have all been used to detect a range of substances from explosives to plant products. For decades the assistance of a working dog to aid in the tracking of narcotics has proven very beneficial to law enforcement.

The introduction of dogs (Canis lupus familiaris) into the daily lifestyle of humans dates back further than 12,000 years ago ${ }^{65}$. The first program to develop dogs for policing purposes began in Ghent, Belgium in 1899 followed by the United States in $1907^{66}$. Although newly developed, these programs did not survive during wars as most dogs were used to aid in various battles. Noted as "war dogs" during the Middle Ages, 
World War I, and World War II, more than 50,000 dogs were used for war purposes ${ }^{65}$. Trained in the art of attack and protected with armor laid across their backs, dogs were used as tactical elements to undergo fire in efforts to draw out the enemies' location. During the First World War it is estimated that more than 7,000 war dogs alone were killed $^{65}$. After understanding the beneficial aspect of dogs, canines (K-9) became more integrated into policing for tracking purposes after the Great War. Following the first modernly organized K-9 program in Massachusetts, canines were used for patrolling work to assistance in the capture of suspects, missing person, and wildlife (unlawfully hunted). The most commonly used breeds used in police work include the German shepherd, Labrador retriever, Belgian Malinois, Rottweilers, Doberman Pinchers, and Bloodhounds ${ }^{66,67}$.

Presently, there are thousands of canine teams deployed for detection of narcotics, explosives, cadaver, live humans, arson, biological threats, money, and various forms of agricultural products ${ }^{68-70}$. To date, research involves understanding and expanding the detection of mold, fungi, narcotics, explosives, currency, and human epileptic seizures. Deemed the "Gold Standard" for detection, canines are efficient, cost effective, fast, easy to train, and are more sensitive than instrumental detection devices ${ }^{68}$. A major advantage for canine detection is the dog's ability to locate a target scent while simultaneously ignoring all interfering non-targeted odors ${ }^{71}$.

\subsubsection{Canine Olfaction and Mechanism}

The superiority of canine olfactory to that of human is vastly understood in the sophistication of their sensory system. Canines have a well-developed olfactory system; 
microsmatic organisms, like dogs, rely on many olfactory cues. In contrast, humans and most birds are microsmatic organisms. The canine sense of smell is 100 times more sensitive than humans and detects odors 10,000 to $1,000,000$ million times below the threshold of humans ${ }^{72,73}$. Canines achieve this by employing a surface area for olfactory cells of $150-170 \mathrm{~cm}^{2}$ to more than 200 million cells depending on the breed in comparison to a human $\left(5 \mathrm{~cm}^{2} \text { and } 5 \text { million }\right)^{71}$. Such a large olfactory area explains why when a human deems a substance odorless, a canine still possesses the ability to detect and also discriminate that same odor.

Understanding the anatomy and working mechanism of the canine's olfactory system is still being studied as more observations bring forth detail to this complex system. Understanding the full ability of a canine's sense of smell begins with the anatomy, Figure 9.

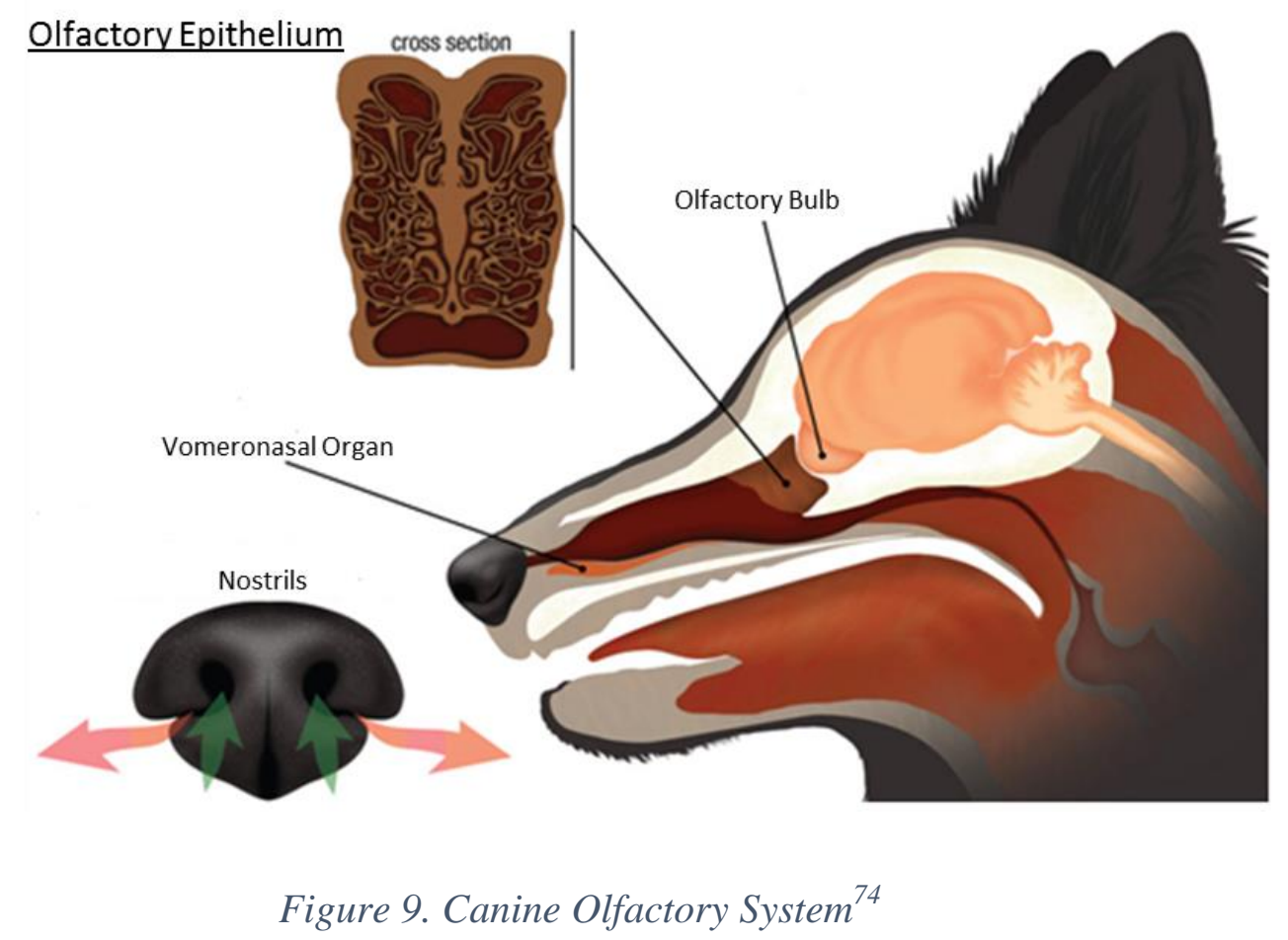


When the surrounding air enters into the airway of a canine there are two pathways it can take: the respiratory or the olfactory epithelium ${ }^{72}$. The respiratory pathway's sole purpose is to breathe in oxygen. After nasal entry the exhaled air is passed through the side slits of the canine's nose. Thus all exhaling air does not interfere with and cause dilution of the inhaled odors. The only organ within the olfactory epithelium that is not used for detection is the vomeronasal organ. This organ detects the pheromones produced by humans and other animals when the nose is wet. The nasal chamber of the olfaction epithelium houses all the organs used for detection. Maxillary and ethmoturbinates are boney ridges whose epithelium is covered in mucus manipulating the movement of air. Within these turbinates there are millions of olfactory cells possessing long and short filaments called cilia which are connected to nerve endings.

When a canine undergoes active sniffing (inhaling short breathes) approximately $300-480$ breathes are taken per minute (frequency $5-8 \mathrm{~Hz})^{71,73,74}$. Inhaling an estimated 3.6L/min, the odorous compounds flow through the nostril into the olfactory organs. These compounds interact with the watery mucus within the organ that contains a binding protein. The compounds, which attach to the protein, are transported through the mucus to the olfaction receptors. These compounds engage with specific cilia of the olfactory cells producing what is called a nerve impulse. The impulse created from all engaged cells travel through the nerve and enter directly into the outer olfaction lobe (cortex $)^{71}$. Within the cortex of the bulb cognitive processing occurs. The dog is aware that there is 
an odor and begins to determine whether they recognize that odor. The cortex helps the dog determine whether the odor elicits a learned reaction or not. The limbic system is the non-cognitive process of the canine's olfactory lobe containing the emotions and natural behaviors that arise. Although canines possess a smaller brain in comparison to their human partners their olfactory bulb is $3 x$ times the size of humans ${ }^{74}$.

Activation of impulses within olfaction cells is described as undergoing a "lock and key" relationship ${ }^{72}$. In simplistic terms the compound is viewed in a particular shape. Once the compound finds a sensory cell with the same receptor site shape, they lock in place and generate a nerve impulse. All receptor sites bearing the same "shape" are connected to the same neuron and the impulses combine and travel to the olfactory lobe. Yielding such a variety of olfactory cells canine sensors are ideal for discrimination work required for detection including that of human scent; as human scents have very little difference.

\subsubsection{Canine Training}

With the overwhelming amount of discrimination achieved by using dogs for detection; the first step is training the dog to track odors. A canine's olfactory neurons

live for approximately $30-60$ days before they die and are naturally replaced ${ }^{67,71}$. When a dog has become routinely exposed to a certain odor for detection and rewarded, there is a shift in the neurons produced. Meaning the newer neurons will contain more of the receptor sites of the odors that the canines are routinely encountering; thus increasing precision and accuracy for detection purposes. 
Prior to training, selection of both a good canine and handler are essential for an effective search team. Physical qualities of a good canine include excellent health and vast amount of mobility, good sense of smell, and a healthy mouth(free of disease which can interfere with the canine's olfactory ${ }^{66,75}$. Useful mental qualities of a dog include a placid composure, lively temperament, good searching drive, ability to cope with mistakes if corrected, and mental stability. As an inadequate handler can ruin a potentially good dog, positive traits of a handler are critical. Patience, fast thinking, lack of tension or anger issues are some of the characteristics an individual must display in order to be considered as a handler. Once an ideal team has been established training can commence.

During the early stages of canine training programs there were several varying forms and ideas on the way to accurately train a canine. If canines were trained in so many different manners, units would face the unnecessary risks of having inadequately trained detection teams. In an effort to correct this, working individuals gathered nationally to develop the standard protocol or foundation for properly training any canine for detection work. The Scientific Working Group on Dog and Orthogonal detector Guidelines (SWGDOG) is the partnership between local, state, federal, and international agencies dedicated to improving the reliability, accuracy, consistency of detector dog teams ${ }^{76}$. The guidelines set forth by this group have been used and cited by numerous agencies for year; including the work conducted here.

The Scientific Working Group on Dog and Orthogonal detector Guidelines formulates a consensus for the best practice general guidelines for efficiently training, 
certifying, and documenting canine work. Canine trainers use these guidelines as a foundation for how their training is conducted. The generalized standard for initial training of narcotic detection begins with a competent trainer, who has clearly indicated and structured the curriculum and objective of the training procedure. Training should begin with obedience training where the canine learns to follow the handler's commands ${ }^{77}$. Training requires a predetermined area that permits ample repetition of control and effective searching routines. The canines will learn to give responses that the handlers can interpret. A canine's alert is indicated by a sit or experienced indications noticed from the handler through extensive work (passive or active). During training the detector dog will be exposed to a variety of locations including those similar to real life scenarios. Training for drug detection includes marijuana, heroin, cocaine, methamphetamine, and any other substance required to meet the training objectives ${ }^{78}$. The quantity of the narcotics used should vary by a factor of ten. The initial training process must continue until the detection team is deemed certified or non-certifiable. Certification is an evaluation (supervisor by canine experts) that determines if the training process was successful and the detector team can search and locate the drugs listed above. The test is designed to also evaluate whether the handler can actively understand any behavior change that the canine undergoes during a search. The certification includes all imprinted odors and team must achieve a $90 \%$ in order to pass and deploy into the field $^{78}$.

\subsubsection{Current Hazards to Canines While Training}

The basis on which narcotic detection works is by exposing the drug to the dog for the sniffing action to take place. The dog inhales the odors from the drug and 
associates a required action when the olfactory cells interact with the compounds. However for decades instances have occurred with various dogs overdosing from introduction to these drugs. In 2015 a Tacoma dog accidentally inhaled methamphetamine after completing a search ${ }^{79}$. Experiencing seizures and a body temperature higher than $109^{\circ} \mathrm{F}$ degrees, the dog became unresponsive the next night after monitoring from a veterinarian. Unfortunately, the previous case is one of the common side effects of introducing dogs to these drugs. Not only is it extremely hazardous, death by accidental inhalation is a gruesome experience. The concern for K-9s and the method in which the drugs are introduced for learning purposes has brought about the need for safe alternatives that still yields positive results.

\subsection{Controlled Odor Mimic Permeation System}

Although canine detection remains the fastest and most efficient tool in detection; there has been an increasing need for a deeper scientific understanding in their detection capability and keen specification. During the early stages of the canine program the calibration and detection ability of a dog was never documented. However, unsuccessful challenges within the courtroom shed light on the limited valid quality controlled checks for a canine's detection ability. In addition these challenges called forth the need for documentation of all success and error rates for admissibility within publications and courtrooms. A successful method in providing the scientific backings required for canine reliability and a safer alternative for substance introduction was by using pseudo aids.

Pseudos are odors that mimic the real substance. Commercially available odor scent aids are provided through Sigma Aldrich and the Non-hazardous Explosive for Security 
Testing and Training (NESTT) for both explosive and narcotics ${ }^{80,81}$. A widely accepted and patented method for odor introduction during training is in the form of Controlled Odor Permeation Systems (COMPS). Unlike other commercially available mimics, COMPS uses the active odors identified through headspace collection of the sample for forensic interest and development. Through canine trials, the active chemicals are placed within the training aid and used to mimic the real substance. Through research it was determined that by identifying and developing the active volatile organic compounds instead of the actual parent chemical responsible for the substance's effect, COMPS is a more efficient and accurate replica of the real substance in comparison to other commercially available aids ${ }^{73}$.

As a deployable technique, COMPS is a reproducible source that houses known amounts of the target odor. On the basis of a double developed system, the technology consists of a permeable container made of a polymer material best suited for containment of the odor followed by storage in a non-permeable package, figure $10^{73,82}$. As the inner bag is housed within the outer, saturation occurs during equilibrium to deliver a reproducible vapor flux of the odor. Beyond reproducibility, advantages to the COMPS system also include it lows cost, no external powering source required, production of a known concentration, and prevention of contamination. Storage in the impermeable package prevents multiple odors from penetrating and mixing with the target odor. 


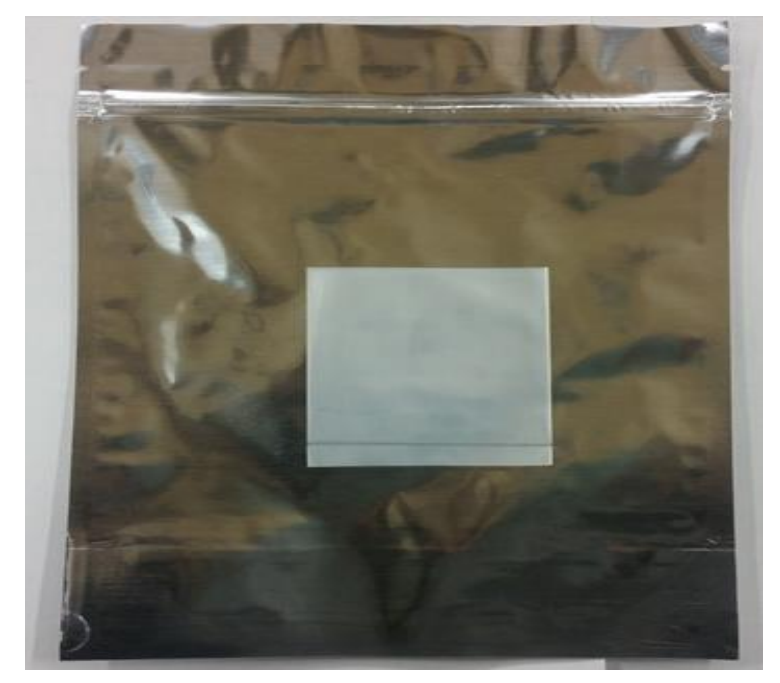

Figure 10.COMPS Setup

\subsection{Analytical Methods}

The analytical examination of various synthetic cathinones was conducted in three essential phases: collection by Solid Phase Microextraction (SPME), separation by Gas Chromatography (GC), and detection by Mass Spectrometry (MS).

\subsubsection{Solid Phase Microextraction}

Solid Phase Microextraction (SPME), developed Janusz Pawlisxyn in 1990, is a sample preparation technique ${ }^{83}$. When developed, routine methods such as Liquid-Liquid Extraction and Solid Phase Extraction (LLE, SPE) were preferred laboratory techniques for analytical experimentation. By eliminating the need to use solvents for matrix removal and analyte collection, SPME reduced the sample preparation time and overall collection of analytes in comparison. Resembling a modified syringe, SPME encompasses a short, thin, rod of fused silica, similar to the chemistry of a GC column. The fiber used is approximately $1-2 \mathrm{~cm}$ long and has an adsorbent layer attached to an elongated rod. The delicate fiber is housed inside of a needle shaft which acts as a 
protective barrier and a piercing tool. Within the hard body of the fiber holder there is a sealing septum, the barrel and adjustment retaining nut to modify the fiber and rod exposure depth, and a plunger (used to expose the actual fiber during sample collection).
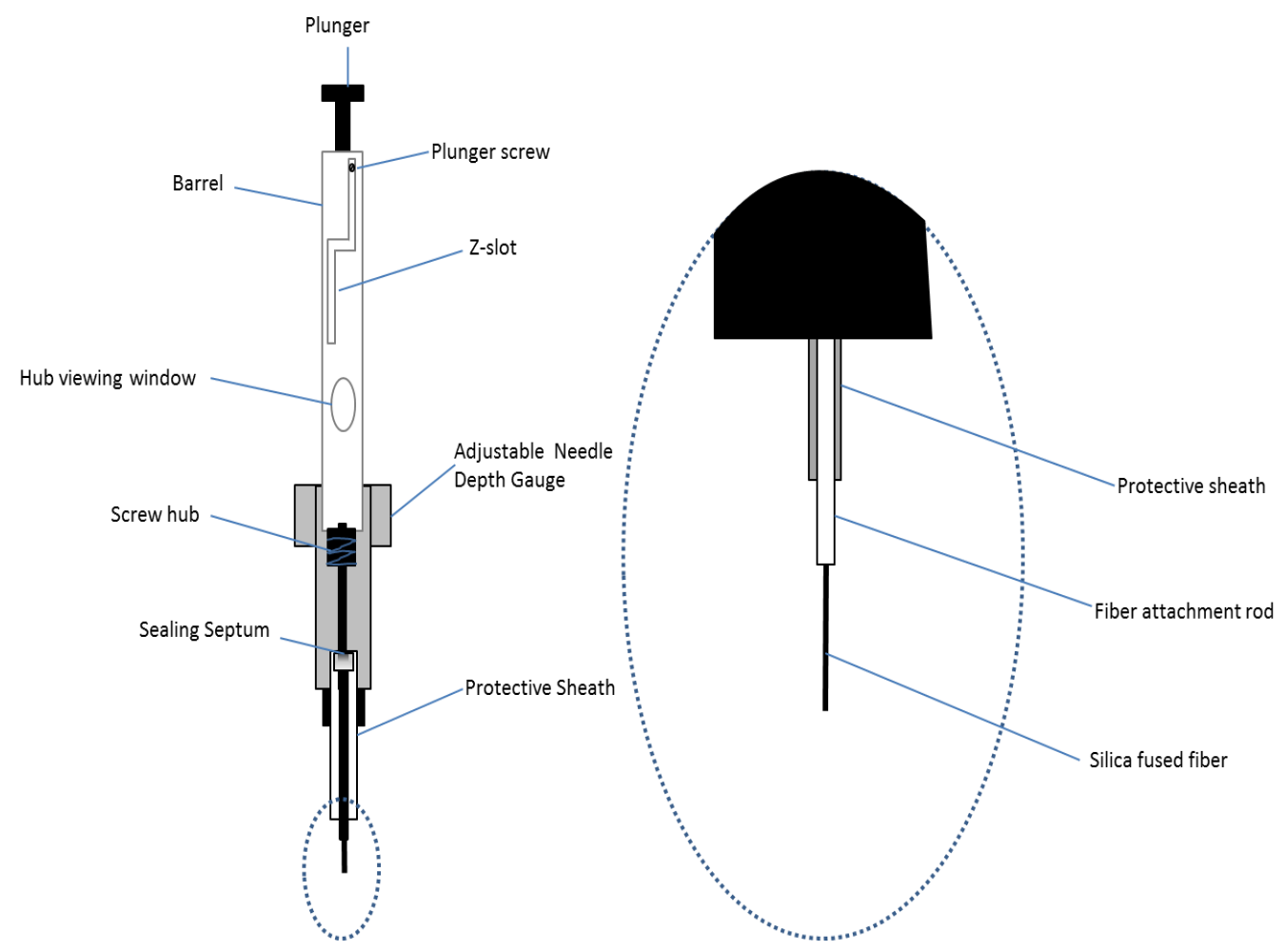

Figure 11. Solid Phase Microextraction (SPME) Fiber Assembly

Solid Phase Microextraction can be performed in two methods: direct immersion which involves a liquid and headspace involving the collection of volatiles. For headspace sampling the VOC analytes are contained in a closed container (i.e., glass vial with a cap) and the needle sheath pierces the septum of the vial. Once stabilized the retaining nut is adjusted to the appropriate depth setting and the plunger is pushed down to expose fiber. Once the predetermined interaction time has commenced the fiber is 
retracted back into the shaft and transferred to the correlating instrumentation for analysis.

SPME extraction is an equilibrium technique that is comprised of two processes: the analytes partitioning between the sample and fiber's material and the concentrated volatiles desorbing from the fiber to an analytical instrument ${ }^{84}$. Although complete extraction of all analytes is not possible with this method, before extraction can begin equilibrium within the system must be established. Equilibrium within a closed vial is developed between three different relationships: (1) the fiber coating to the sample to be analyzed, (2) the headspace above the sample to the solid or liquid substance, and (3) the sample's headspace to the fiber's coating ${ }^{84}$. As depicted by the equation 1 the concentration of the sample (Co) and the volume of the sample utilized (Vs) will be divided amongst the three equilibriums. $C_{h}^{\infty}, C_{s}^{\infty}, C_{f}^{\infty}$ and $V_{h}, V_{s}, V_{f}$ are the equilibrium concentrations and volumes of the headspace, sample, and fiber (respectively).

$$
C_{o} V_{s}=C_{h}^{\infty} V_{h}+C_{s}^{\infty} V_{s}+C_{f}^{\infty} V_{f}
$$

Equation 1. The State of Equilibrium Distributed Throughout the System from the Initial Amount of Analyte Introduced.

For liquid sampling via direct immersion of the SPME fiber, the amount of sample be extracted is notated in equation 2. Direct immersion involves a liquid sample that the SPME directly interacts with to extract analytes. The amount of sample extracted is based on the volume of sample. Solutions larger than $5 \mathrm{~mL}$, where the volume surpasses the volume of the fiber are represented by: 


$$
n_{f}=K_{f s} V_{f} C_{o}
$$

Equation 2. The Amount Extracted During Direct Immersion for Large Volume Samples

The previously expressed equation shows the amount absorbed is directly related to the initial concentration of the sample $\left(\mathrm{C}_{\mathrm{o}}\right)$, the volume of the fiber $\left(\mathrm{V}_{\mathrm{f}}\right)$, and the partition from the sample to the fiber adsorbent $\left(\mathrm{K}_{\mathrm{fs}}\right)$. When a limited amount of sample is analyzed $(2-5 \mathrm{ml})$ the amount absorbed is represented by:

$$
n_{f}=\frac{K_{f s} V_{f} V_{s} C_{o}}{K_{f s} V_{f}+V_{s}}
$$

Equation 3. The Amount Extracted During Direct Immersion for Finite Volume Samples

Equation 3 expresses how the amount absorbed on a fiber can also be reduced by the partition coefficient and fiber volume when compared to the volume of the sample. Headspace sampling involves the extraction of all compounds that have transition into the gaseous phase. This method is useful when dealing with samples containing heavily interfering matrices such as grease and oil. As the extraction of a sample's headspace involves establishing the equilibrium between three phases, the amount of analytes extracted is determined based on the partition between each relationship. Partition coefficients $\left(\mathrm{K}_{\mathrm{fh}}, \mathrm{K}_{\mathrm{hs}}\right.$, and $\left.\mathrm{K}_{\mathrm{fs}}\right)$ are the probability of volatiles or analytes to cross between the two equilibriums (i.e., fiber to headspace, headspace to substance, and fiber to substance) depending on the concentration $\left(\mathrm{C}_{\mathrm{x}}\right)$ of each phase. 


$$
\begin{gathered}
K_{f h}=\frac{C_{f}}{C_{h}} \quad K_{h s}=\frac{C_{h}}{C_{s}} \quad K_{f s}=\frac{C_{f}}{C_{s}} \\
n_{f}=\frac{K_{f s} V_{f} V_{s} C_{o}}{K_{f s} V_{f}+K_{h s} V_{h}+V_{s}}
\end{gathered}
$$

Equation 4. The Partition Coefficients between Each Phase and the Amount of Analyte Collected on a Fiber during the Headspace Process

Using on equation 4, the amount of analyte collected is not influenced by the sample extracted during direct interaction or through headspace, however determined greatly by the volumes. The volume of the fiber, the headspace, and the samples itself have the greatest influence on the amount collected ${ }^{84,85}$. For example, increasing the fiber volume allows more analytes to adsorb or absorb to the fiber, increasing the volume of the headspace will decrease the amount extracted, and the volume of sample influences the extractable portion in various manners. A larger contributing factor that can be optimally promoted for beneficial reasons is the partition coefficient between the fiber's coating and the sample $\left(\mathrm{K}_{\mathrm{fs}}\right)$; discussed later.

Through experimentation it has been determined that analytes with larger $\mathrm{K}_{\mathrm{fs}}$ tend to have a greater binding affinity to the fiber than the sample, thus making them more likely to be extracted ${ }^{84,85}$. In efforts to increase the likelihood of a compound's adsorption/absorption to a fiber, there are several parameters that can be ideally selected to enhance the partition. One of the easiest ways to increase an analytes partition is by selecting a fiber chemistry that includes a higher coefficient for the targeted analyte. 
Although the first commercially available fiber phase consisted of polydimethylsiloxane (PDMS), there are currently several fibers with varying stationary phases.

Table 4. The Commercially Available Fiber Coatings and Their Ideal Analyte Type Extracted $^{86}$

\begin{tabular}{|c|c|c|}
\hline Phase & Thickness & Application \\
\hline Polydimethylsiloxane (PDMS) & $\begin{array}{c}7 \mu \mathrm{m} \\
30 \mu \mathrm{m} \\
100 \mu \mathrm{m}\end{array}$ & $\begin{array}{c}\text { Non-polar semi } \\
\text { volatiles } \\
\text { MW 125-600 }(7 \mu \mathrm{m}) \\
\text { MW 80-500 }(30 \mu \mathrm{m}) \\
\text { Volatiles MW 60-725 } \\
(100 \mu \mathrm{m})\end{array}$ \\
\hline $\begin{array}{c}\text { Polydimethylsiloxane/Divinylbezene } \\
\text { (PDMS/DVB) }\end{array}$ & $\begin{array}{l}60 \mu \mathrm{m} \\
65 \mu \mathrm{m}\end{array}$ & $\begin{array}{c}\text { Amines and polar } \\
\text { compounds }(60 \mu \mathrm{m}) \\
\text { Volatiles, amines, and } \\
\text { nitro aromatic } \\
\text { compounds }(65 \mu \mathrm{m})\end{array}$ \\
\hline Carboxen/Polydimethylsiloxane (CAR/PDMS) & $\begin{array}{l}75 \mu \mathrm{m} \\
85 \mu \mathrm{m}\end{array}$ & $\begin{array}{l}\text { Gases and low MW } \\
\text { compounds }\end{array}$ \\
\hline Polyacrylate (PA) & $85 \mu \mathrm{m}$ & $\begin{array}{l}\text { Polar semi-volatiles } \\
\text { (MW 80-300) }\end{array}$ \\
\hline $\begin{array}{l}\text { Divinylbezene/Carboxen/Polydimethylsiloxane } \\
\text { (DVB/CAR/PDMS) }\end{array}$ & $50 / 30 \mu \mathrm{m}$ & $\begin{array}{l}\text { Flavor compounds } \\
\text { (volatile and semi) }\end{array}$ \\
\hline Polyethylene Glycol (PEG) & $60 \mu \mathrm{m}$ & $\begin{array}{c}\text { Alcohols and polar } \\
\text { compounds (MW 40- } \\
725 \text { ) }\end{array}$ \\
\hline
\end{tabular}

Polydimethylsiloxane uses a non-polar stationary phase to bind compounds such as aromatics, esters, and many pesticides. Polydimethylsiloxane/ Divinylbezene (PDMS/DVB) is a semi-polar phase which operates in the collection of amines; while phases like Polyacrylate (PA) and Polyethylene Glycol (PEG) are used to extract polar compounds such as phenols and highly polar compounds like alcohols (respectively). Along with alternative stationary phases, the thickness can also vary between and within 
each fiber type. Fiber thickness can help increase sensitivity by allowing a different range of molecular heavy compounds to be selected (however increasing thickness increases the equilibrium time) or as a restriction for the type of analysis conducted. Although the $60 \mu \mathrm{m}$ PDMS/DVB fiber can only be used during High Performance Liquid Chromatography, the $65 \mu \mathrm{m}$ thickness can be used for both solvent and solvent-less (headspace) extractions ${ }^{86}$. Determining the most suitable fiber phase for collection of analytes is the first variable that can easily be determined to increase the partition from the sample to the fiber.

Beyond selection of an optimal polymer the extraction speed, equilibration time, and temperature are all factors that influence the sensitivity and successfulness of the SPME process. The extraction speed that commences during the exposure of a polymer to the surrounding headspace is the rate at which the analytes leave the matrix and bind to the fiber ${ }^{83-85}$. Depending on the distribution coefficient, the amount of time required to optimally allow the maximum number of compounds to extract must be determined. The setting is determined when the amount of analytes extracted has plateaued. As displayed in equation 1 , headspace collection involves the established equilibrium state among all relationships. Determining the equilibration period is the time required for the rate of analytes transitioning from the sample to headspace, the sample to the fiber, and the headspace to the fiber (and vice versa) to become constant ${ }^{84}$. As SPME is an equilibrium technique for the collection of any desired analyte to begin, stability must be developed between the sample and headspace and then again with the fiber to allow the compound to distribute between each. Heating the sample, has added benefits to SPME by increasing its sensitivity. Applying heat during the extraction process allows for the less 
volatile compounds to release in the headspace (thus promoting collection) and increases the analyte concentration within the area ${ }^{84,87,88}$. However, with applying heat there is also a decrease in the lower boiling components, thus the range used during the extraction process must be monitored to determine the point of significant loss.

Once the volatiles are absorbed to the polymer of the fiber, desorption via an analytical instrument is necessary. Routinely used in crime labs and various research facilities, Gas Chromatography (GC) is often paired with SPME to thermally desorb the compounds from the fiber. Once the extraction has completed the fiber is taken to the injection port of a GC. The fiber polymer is exposed inside the inlet at a certain temperature (operating inlet temperature range is indicated on supplementary information from manufacture). The allotted amount of time for the fiber to remain exposed in the inlet is known as desorption time. During this period the compounds form a greater partition coefficient to the gas as the temperatures increases, thus desorbing off the fiber onto the column. Determining the appropriate length of time is the last essential step in volatile extraction to ensure that all analytes have desorbed; preventing carryover (residual compounds still absorbed to the polymer).

\subsubsection{Gas Chromatography}

Chromatography is defined as the art of separating a mixture composed of various analytes. The technique works on the basic premise that analytes will vary in their partition coefficient between a given stationary and mobile phase. Developed in 1942 by John Martin and Richard Synge, partition chromatography was achieved by employing two liquids, one acting as the stationary element absorbed to an inert support and the 
other as the mobile phase ${ }^{89}$. Ten years later the advantageous idea of using a gas as the mobile phase was explored by Martin alongside Anthony T. James; known as gas chromatography. Since its development Gas Chromatography (GC) has been pivotal in the separation of various samples both of a known and unknown quality. As the name suggests, GC uses a carrier gas as the mobile phase and either a liquid on an inert support or a solid substance as the stationary phase (later more common) engaging with the components of the volatile mixture to separate them. The working principle of GC is as the sample is introduced onto the column a carrier gas will push the sample through. Depending on the distribution of the sample (equation 5) and its boiling point, the sample's components will separate depending on each compound's likelihood to bind to the column or the carrier gas. The separated analytes elute into the detector where a chromatogram is produced. A chromatogram is a detector signal displayed on a potentiometric recorder where the intensity is plotted versus time ${ }^{89,90}$.

$$
K=\frac{C_{s}}{C_{m}}
$$

Equation 5. Distribution Coefficient between the Stationary and the Mobile Phase

The general apparatus design of a standard GC instrument is composed of several different working parts: carrier gas/tank, pressure controller, injector, GC oven, column, and detector (figure 12). As previously explained the purpose of the carrier gas is to transport the analyte/solute through the column for separation. Although there are various types of gas that can be used without deterioration of the column (except for oxygen); the carrier gas ultimately used is decided by the type of detector coupled to the $\mathrm{GC}^{90}$. 
Commercially available gases include argon (for high separating power), hydrogen and nitrogen (with a flame ionization detector), and carbon dioxide (for critical phase chromatography). The most universally used gas in a GC system is helium. As a preferred gas, helium has the highest thermal conductivity of all permanent gases and is not combustible (like hydrogen $)^{90,91}$. Although it can be expensive to purchase, helium allows for swift analysis to be conducted without clogging the column. The flow controller is responsible for the reducing the pressure of the gas before it enters into the GC system. In order to maintain a constant flow rate the pressure/flow controller, located before the column, automatically increases the pressure within the system throughout the run.

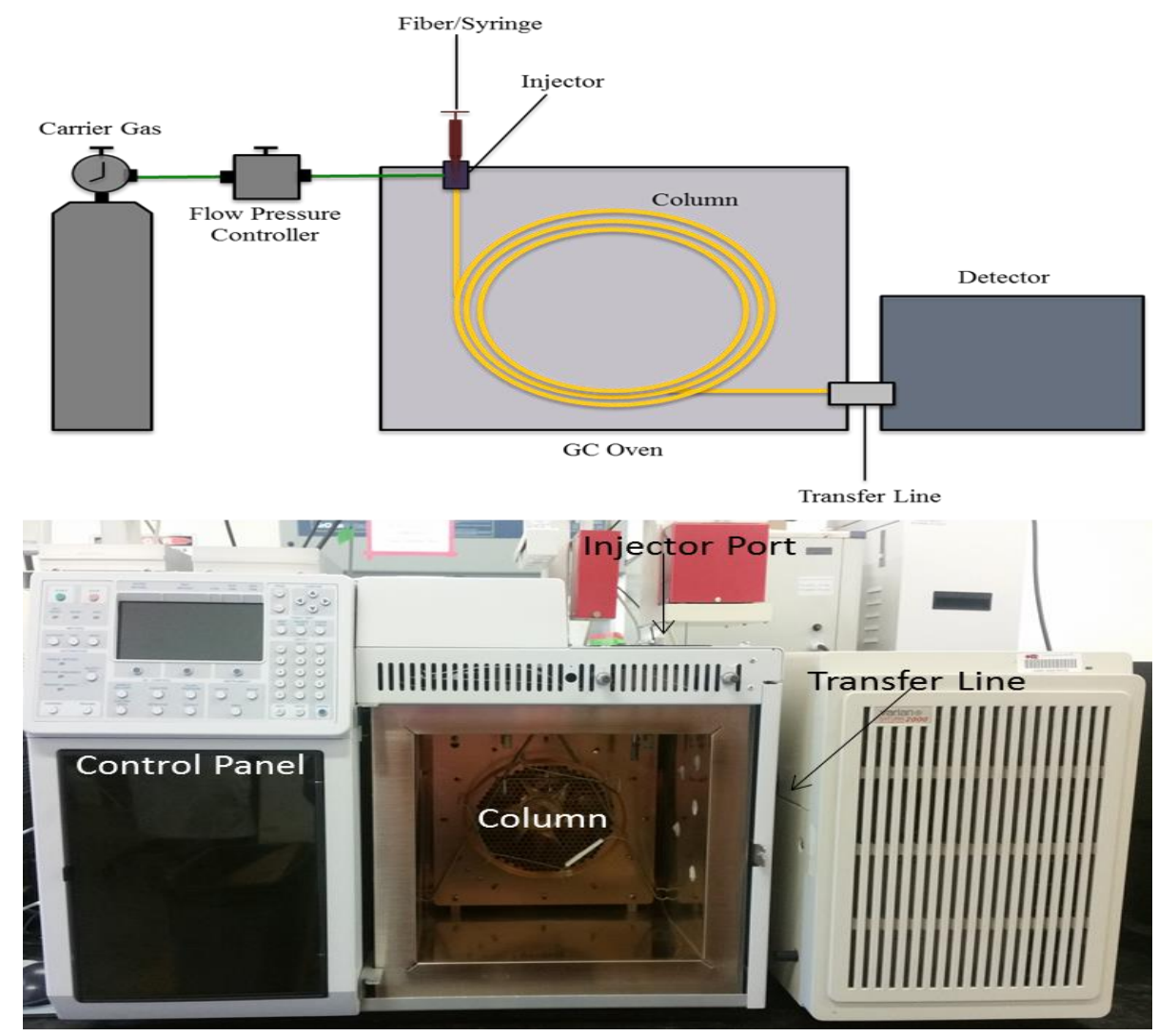

Figure 12. Gas Chromatography Apparatus Configuration 
The injector site of the apparatus is where the syringe or SPME fiber is introduced. The injector is composed of a thermally elevated space (liner) and a septum $^{90,91}$. The fiber pushes through the rubber septum and the sample is released in the heated space above the column or via on-column injection. In the heated zone above the column the sample is flash vaporized or the gases are desorbed off the fiber. In the inlet a process called splitting is also observed, where only a fraction of the sample introduced actually goes to the column. The excess is pushed into a waste channel. On-column injection involves penetrating the column $0.5-1$ inches in deep and releasing the solute onto the first plate of the column ${ }^{90}$. Solid Phase Microextraction desorption occurs in the heated zone directly above the column (column penetration is not possible) where the helium gas pushes to transport the solute through the column. The GC oven houses the column. Temperature programming is the main influencing factor for compound separation. Heating the system is achieved by cycling warm air through a turboheater. Manipulation of the temperature (increasing and holding a certain degree for an extended period of time) has the benefit of speeding the runtime to achieve an analyte's boiling point faster or holding it; allowing for a more precise resolution of the analyte peaks. Most chromatographic systems allow settings to range from $50-500^{\circ} \mathrm{C}$ (restricted by the maximum operating temperature of the column) and various ramping rates to achieve the desired chromatographic run.

There are essentially two types of gas chromatographic columns: packed and open tubular (capillary) $)^{89,92}$. Packed columns are stationary phases composed of glass, metal, 
stainless steel, aluminum, and nylon that do not possess a center hollow. Columns of these types are equipped to handle a larger sample capacity. Capillary columns use an internal diameter varying in sizes, where the mixture interacts with the stationary phase. As a result of its high resolving power capillary columns yield greater separation for complex mixtures and narrower peaks; yet the sample capacity is low ${ }^{93,94}$. Regardless of the column types selected each houses a stationary phase (polar or non-polar) directly engaging in the separation process.

The optimum column and stationary phase chosen should be indestructible at higher temperatures, thermally and chemically stable, and resistant to oxidation ${ }^{93,94}$. When selecting the appropriate stationary phase it is important to remember the type of forces at play in the separation process: dipole-dipole, dispersion, hydrogen bonding, and polarity. Appropriate column selection is determined by the principle "like dissolves like", where maximum separation is accomplished by choosing a phase whose chemical structure is similar to the compounds potentially extracted. Yet if the sample is unknown, testing out a nonpolar and polar stationary phase and comparing the resulting chromatograms, will aid in the correct selection of the column. To date there are a variety of different columns and stationary phase used for both qualitative and quantitative analysis. These columns vary in both length and film thickness (material in column $)^{90,92,94}$. 
Table 5. Types of Column Stationary Phases used in Gas Chromatography

\begin{tabular}{|c|c|c|}
\hline Stationary Phase & $\begin{array}{c}\text { Maximum } \\
\text { Operating } \\
\text { Temperature }\left({ }^{\circ} \mathrm{C}\right)\end{array}$ & Application \\
\hline Polydimethylsiloxane & 350 & $\begin{array}{c}\text { Non-polar, aromatics, } \\
\text { hydrocarbons }\end{array}$ \\
\hline Poly(phenylmethyldimethyl) siloxane & 350 & $\begin{array}{l}\text { Fatty acids methyl } \\
\text { ester, halogenated } \\
\text { compounds }\end{array}$ \\
\hline Poly(phenylmethyl) siloxane & 250 & $\begin{array}{l}\text { Drugs, steroids, } \\
\text { pesticides }\end{array}$ \\
\hline Polyethylene glycol & 250 & $\begin{array}{l}\text { Free acids, alcohols, } \\
\text { esters, amines }\end{array}$ \\
\hline Poly(dicyanoallydimethyl) siloxane & 240 & $\begin{array}{l}\text { Polyunsaturated fatty } \\
\text { acids, free acids, } \\
\text { alcohols }\end{array}$ \\
\hline $\begin{array}{l}\text { Poly(trifluoropropyldimethyl) } \\
\text { siloxane }\end{array}$ & 200 & $\begin{array}{l}\text { Chlorinated } \\
\text { aromatics, } \\
\text { nitroaromatics }\end{array}$ \\
\hline
\end{tabular}

Once the compounds have eluted from the column, they continue into the detector. The detector is where the compounds are converted into a detected signal and the signal is plotted versus the length of time required for the analyte to reach the detector $^{90}$. The chromatogram produced shows peaks corresponding to the solute identified. Peak resolution is the degree of separation between two compounds as they elute from the column ${ }^{90,91,94}$. If resolution is less than 1 , then temperature programing can be manipulated until an acceptable resolution is achieved. Choosing the most suitable detector for analysis is one that has rapid response to the presence of any analyte, high 
sensitivity, stability, robustness, accurate linear response to increasing concentrations, can withstand changes to high temperatures and flow rate, while giving accurate responses to all compounds detected ${ }^{91,95}$. Experimentally used detectors include flame ionization, argon and micro argon, electron capture, nitrogen phosphorus, and a thermal conductivity detector. Modern day research and analysis routinely employ the use of a Mass Spectrometric (MS) detector coupled to a $\mathrm{GC}^{90,94,95}$.

\subsubsection{Mass Spectrometry}

Mass Spectrometry (MS) is the analytical technique that involves the ionization, separation, and detection of various analytical specimens. Developed more than 100 years ago, MS has been instrumental for its unequalled sensitivity, speed, diversity of analysis, and detection limits ${ }^{85}$. Mass Spectrometry is a vacuum technique that involves three major components: ion source, mass analyzer, and detector (figure 13).

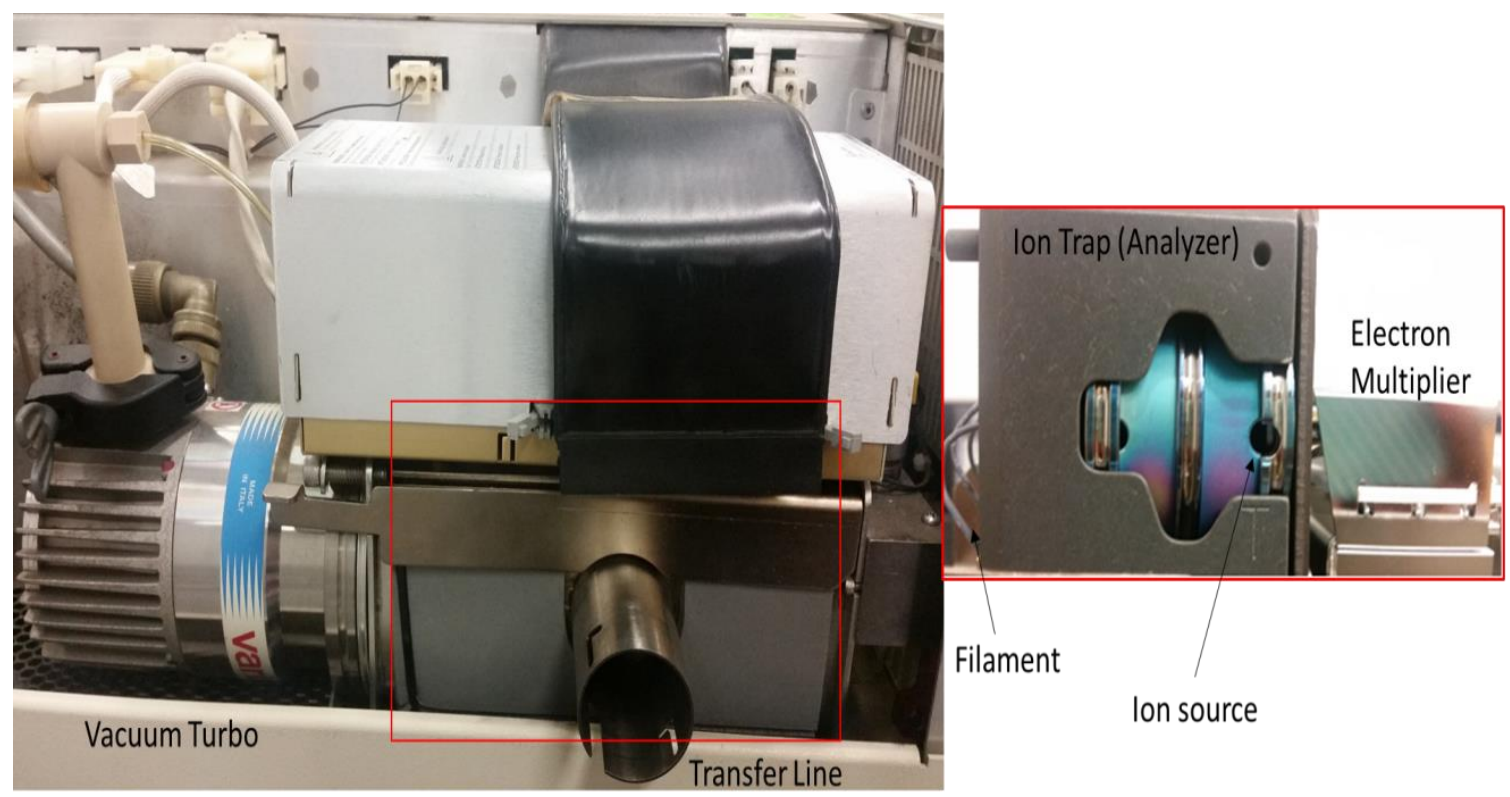

Figure 13. Mass Spectrometry Apparatus Configuration 
When a sample is introduced into the ion source (through a GC) the separated compounds entered through a transfer line. The transfer line is preset at an elevated temperature as the analytes enter into the vacuum system and the ion source. The vacuum system of the mass spectrometer is a necessity for the system to operate functionally. By providing a vacuum system this prevents the ions that are formed from colliding with each other; causing unwanted reactions and products. These collisions can result in the ions hitting the walls and losing their charge as well as reaction products that complicate the resulting mass spectrum ${ }^{96}$. An efficient vacuum pressure is established and maintained with mechanical pumps including turbo molecular, diffusion, and/or cryogenic pumps.

Upon entering the MS compounds are directed into the ion source. The ion source is responsible for ionizing the analyte so that it bears a charge that can be detected. The most typical ion source procedure is Electron Ionization (EI). This ionization process involves a heated filament which gives off electrons accelerating towards an anode. During acceleration the electrons collide with the molecules (M) entering from the GC to produce ions that move on to the analyzer; equation 6 .

$$
M+e^{-} \rightarrow M^{++}+2 e^{-}
$$

Equation 6. Electron Ionization Process for Molecules

The energy of an electron for EI is $70 \mathrm{eV}^{96}$. Each electron's wavelength is correspondent to a certain bond length within a molecule. When energy is close enough to a transitional state within a molecule it will transfer its energy to the analyte. The amount of energy transferred can range from $10-20 \mathrm{eV}^{96}$. The energy required to ionize a 
molecule is approximately $10 \mathrm{eV}$, so the excessive energy the molecule possesses leads to extensive fragmentation. The severity of fragmentation during EI ensures that the whole molecular structure of the compound is lost; thus determination of unknown compounds can be difficult.

As electron ionization is a more energetic technique it results in more fragmenting of the molecule in question ${ }^{97}$. There are soft techniques such as Chemical Ionization (CI) that produce more ions similar to the molecular species as a whole. Chemical ionization is a soft ionization technique which allows the molecular ion (molecular weight) of the species to be identified. As a complimentary technique to EI, CI assists with determining the unknown component by providing the molecular mass. Chemical ionization works by colliding the molecules to be analyzed with primary ions within the source instead of directly with electrons ${ }^{98}$. A reagent gas interacts with the electrons directly to produce charged primary ions. These primary ions remain in the ion source to interact with the sample as it enters the source from the GC. Although there are several types of ionization process that may occur (adduct formation, charge-transfer, etc.) the most common is proton transfer; equation $7^{99}$. Similar to an acid-base reaction, the molecule (M) acts as the proton acceptor (base) and the gaseous ion $\left(\mathrm{GH}^{+}\right)$as the donor (acid). 


\section{1. $G+e^{-} \rightarrow G^{\cdot+}+2 e^{-}$ \\ 2. $G^{++}+G \rightarrow G H^{+}+G^{\cdot}$ \\ 3. $G H^{+}+M \rightarrow M H^{+}+G$}

Equation 7. Chemical Ionization Process. Where Step 1 is the Ionization of the Reagent Gas. Step 2 is the Secondary Reaction that Produces Primary Protonated Reagent Ions. Step 3 is the Interaction between the Reagent Ions and Molecule to Produce a Protonated Molecule

The transfer of proton from one source to the next is determined by proton affinity $^{96,97}$. The term defines how likely the molecular species is to possess the proton. If the analyte has a larger proton affinity than the gaseous ion and the reaction is an enthalpic one, the transfer will occur. Overall the energy transferred during the ionization phase is only a fraction of EI and the resulting spectrum allows the molecular ion to be easily recognized. There are several reagents that can be used for chemical ionization; however the type of mass spectrometer a facility is equipped with dictates the reagent used. Typically methane, isobutane, and ammonia are all reagent gases that can be employed for chemical ionization ${ }^{97,100}$. However certain systems use the gas depleted from a reagent liquid to conduct chemical ionization. These reagent liquids include methanol, ammonia, and acetonitrile. Currently there are several types of chemical ionization techniques including desorptive and atmospheric chemical ionization.

Once the gaseous phase-molecular interaction has occurred, ions are transmitted to a mass analyzer where they are separated. Within a mass analyzer the separation is 
determined by the mass of the ions $(\mathrm{m})$ as well as the charge $(\mathrm{z})$; a ratio $(\mathrm{m} / \mathrm{z})$ developed. Thus large ions which possess more than one charge will not be equivalent to their suspected molecular weight. Although there are a variety of mass analyzer, each with their own advantages and disadvantages, all separate ions on the basis of on magnetic fields and/or static and dynamic electric fields.

\section{Table 6. Types of Mass Spectrometric Analyzers}

\begin{tabular}{|c|c|}
\hline \multicolumn{2}{|c|}{ Type of Analyzer } \\
\hline Quadrupole & $\mathrm{m} / \mathrm{z}$ (trajectory stability) \\
\hline Ion trap & $\mathrm{m} / \mathrm{z}$ (resonance frequency) \\
\hline Time-of-flight & Velocity (time flight) \\
\hline Magnetic Sector & Momentum \\
\hline Fourier transform ion cyclotron resonance & $\mathrm{m} / \mathrm{z}$ (resonance frequency) \\
\hline Fourier transform orbit trap & $\mathrm{m} / \mathrm{z}$ (resonance frequency) \\
\hline
\end{tabular}

Generally, analyzers operate in two different fashions. Scanning analyzers will separate ions in a time scale only allowing a certain $\mathrm{m} / \mathrm{z}$ to pass (quadrupole) while other analyzers (ion traps) allow the transmittance of all ions and separate them on the basis of oscillating factors ${ }^{96}$. The quadrupole is a device comprised of four perfectly parallel rods that separate ions on the basis of trajectories in oscillating electric fields ${ }^{96}$. Described initially at Bonn University, the quadrupole system allows an ion to enter the system traveling along the z-axis. The potentials applied to the rods will cause the ions to fluctuate and traveling towards to the rods (either along the $\mathrm{x}$ or $\mathrm{y}$ axis). As the ion is approaching the rod and before it strikes the surface, the potential associated with that rod changes, pushing the ion in a different direction. The forces applied in the electric fields result in changes in the trajectory of the ion, while the stability of the ions along these different axes relationships allow separation to commence using the $\mathrm{m} / \mathrm{z}$ ratio of the ion. 
The quadrupole ion trap (commonly referred to as Paul Ion Trap) was invented in 1960 by Paul and Steinwede ${ }^{96}$. The 3D structure, most effectively used, is composed of a circular electrode with two ellipsoid caps located at the top and bottom (essentially a quadrupole bent in on itself). Unlike the quadrupole where only a certain mass is allowed through the rods, ion traps will house all ions of all masses inside. The masses of the analyte are ejected from the trap according to their mass and a spectrum is obtained. A large component of the working principle of ion traps is maintaining a stable trajectory of all ions housed in the analyzer. As the ions repel each other, the designed trajectory will expand overtime. Expanded trajectories run the risk of collision with the walls of the trap. Prevention of such collisions is achieved by operating the trap at a pressure around $10^{-3}$ Torr $^{96}$. Similar to quadrupoles the ion trap will apply a potential on the electrodes supporting the expulsion of certain ions.

Once a generated ion passes through the analyzer, they are detected and transformed into a signal that correlates directly to their abundance. Although there are several detectors available, the types used is dependent on the mass spectrometer commercially employed. To date, the most widely used detector is the Electron Multiplier (EM). The EM works by increasing the acceleration of the ions that enter from the mass analyzer, enhancing the detection efficiency. The ions will collide with a conversion dynode (potential \pm 3 to $\pm 30 \mathrm{kV}$ ) of the opposite charge to produce secondary particles ${ }^{96}$. Ions which possess a positive charge will strike a dynode bearing a negative charge to produce negative ions, neutrons, and electrons. Any ion that bears a negative charge will produce the opposite charge with a positive dynode. The secondary particles will convert into electrons and collide with additional dynodes in a cascading effect to amplify the 
electrons and produce a signal. An interface receives the signal and digitizes it to a computer in such a format that can be displayed and interpreted.

\subsection{Statistical Analysis}

Statistics is defined as the mathematical science which uses quantitative examination to analyze and present numerical data collected during the experimentation process ${ }^{101}$. Essentially, statistical analysis is used to scrutinize all data values collected from the sample and test a hypothesis. The goal of statistics is to describe a sample as it relate to its mean or generalize it as a representation of a population ${ }^{101}$. Using statistical analysis helps verify or reject the validity of a method, develop a correlation between the sample and the represented population, conduct predictive analysis for future experimental actions, and analyze the error encountered during scientific experiments. There are several methods under the realm of statistical analysis that can be employed depending on the data to be analyzed and the overall goal of the experiment.

\subsubsection{Statistical Significance Test (T-test)}

During experimentation, there are two general types of errors that can be encountered: systematic and random. While systematic error will skew the results generally in one direction, random error will causes fluctuations either direction. Regardless of the error type values measured from the sample may deviate from the true values. In order to ensure that the calculated deviation between the analyte's amount and the true value are accurate, one must be completely unbiased. Statistical significance test (t-test) is used to account for the potential that random error has occurred between the observed value and the standard value; i.e. whether the difference can be summed up to 
random error (uncontrollable therefore unavoidable) or are the two values significantly different. In order to do so the null hypothesis that there is no systematic error impacting the results must be tested. With this theory you can test whether the observed variation of the mean $(\mathrm{x})$ and true value $(\mu)$ is caused by random errors; equation 8 . If the probability of this occurring is less than 1 in 20 or $5 \%\left(\mathrm{P}=0.05\right.$ level) then the null is rejected ${ }^{102}$. When determining the error between mean values of one experiment the following equation is used: where $n$ is the sample size and $s$ is the sample standard deviation.

$$
t=(x-\mu)^{\sqrt{N}} / s
$$

Equation 8. Significance T-test for Mean Value Variation; where the $t$ value is Determined by the Mean (x), True Value $(\mu)$, Sample Size $(N)$ and Standard Deviation ( $s)$

Once the $t$ value is determined it is compared to a critical value located in a standardized chart, by degrees of freedom. The degrees of freedom when looking at mean values is subtracting the sample size by one. If the t value is larger than the critical value then the null hypothesis is rejected; meaning there is significant difference. When comparing two experimental methods the previously used equation is not sufficient enough to account for all variations. Testing the null hypothesis of two experimental methods is to determine whether they give the same results as the true values; therefore $\mathrm{x}_{1}-\mathrm{x}_{2}=0$. Because two different methods will have different standard deviations, the pooled estimated deviation must be calculated first before determining the value of $\mathrm{t}$. 


$$
\begin{gathered}
s=\frac{\left(n_{1}-1\right) s_{1}^{2}+\left(n_{2}-1\right) s_{2}^{2}}{\left(n_{1}+n_{2}-2\right)} \\
t=\frac{x_{1}-x_{2}}{s \sqrt{\frac{1}{n_{1}}+\frac{1}{n_{2}}}}
\end{gathered}
$$

Equation 9. Significance T-test for Variation between Two Methods; where the t value is Determined by the Mean (x), True Value (u), Sample Size (N) and Standard Deviation (s)

As with mean values, if the $t$ calculated is larger than the critical value the null is rejected and the two methods do bear significant differences. The t-test is one of the simplest yet efficient types of statistical analysis employed.

\subsubsection{Analysis of Variance (ANOVA)}

The primary limitation presented from t-test is the maximum number of methods the deviations can detect is two. Outside of random error variations between several methods can also be accounted for by controlled factors. These factors include conditions of storage, differing analytical methods, testing different parameters between methods, and many more. In order for successful separation of estimation of variations not caused by systematic error, a stronger statistical technique is required. Analysis of Variance (ANOVA) is a statistic tool used to test whether changing controlled factors will lead to a significant difference between the mean values observed between several methods ${ }^{102}$. When there is one controlling factor outside of random error then a one-way ANOVA test is used. In this type of ANOVA the variations within and between multiple samples 
are compared to determine if they are significantly different. The F-test employed to calculate the change is shown in equation 10.

$$
F=\frac{\frac{n \sum_{i}\left(x_{i}-x_{j}\right)^{2}}{(h-1)}}{\frac{\sum_{i} \sum_{j}\left(x_{i j}-x_{i}\right)^{2}}{h(n-1)}}
$$

Equation 10. ANOVA Formula for Variation of Several Methods within and between; where the Sample Number ( $n)$, Mean (x), Samples ( $h)$ Determine the F-test value

The numerator indicates the sum of squares divided by the degrees of freedom (h1) to showcase the variation between samples, while the denominator indicates the variance occurring within the sample. The $F$ value that is determined it compared to an $\mathrm{f}$ critical value, determined in a summarized chart similar to the t-test. If the F calculated is larger than the critical then the null is rejected and there is significant difference between the means values across the several methods due to a larger in-between variation. Twoway ANOVA is used to not only test the variation of the factor experimental modified but also other uncontrolled factors outside of random error affecting the results by causing a trend. This includes the day sampling was perform, the temperature, the varying amounts of samples used each time the experiment was performed. In addition to testing whether there is significant difference between numerous methods, two-way ANOVA will also test whether the variations provided by the uncontrolled factors can be summed up to random error, or is it significantly different. Determining the type of ANOVA test to be used is helpful to eliminate any biasness that may occur once results have been collected. 


\subsubsection{Predictive Values}

When comparing results to a golden standard (ideal) of canine detection, the method of predictive values is useful. Predictive value is a statistical technique that tests whether the positive or negative values obtained are true. This tool can be useful to support whether or not the observed positive or negative findings (i.e. canine alerts) are indeed the most accurate results based on the gold standard. As the name suggest Positive Predictive Value and Negative Predictive Value (PPV, NPV respectively) test the likelihood of the results truly being positive or negative based on the prevalence, specificity, and sensitivity of the measured values ${ }^{103}$.

$$
\begin{gathered}
P P V=\frac{\text { true positive }}{\text { true positive }+ \text { false positive }} \\
N P V=\frac{\text { true negative }}{\text { true negative }+ \text { false negative }} \\
\text { Equation 11. Predictive Value Statistical Testing to Determine the Validity and } \\
\text { Likelihood of Result }
\end{gathered}
$$

Each calculated value represents the probability of the positive and negative being true; where the closer to $100 \%$ the likelihood that the observed values being valid increases. As a supporting statistical test, predictive values have been used to determine the likelihood of various diagnostic tests as well as in various canine detection studies ${ }^{82,104}$. 


\section{Significance of Research and Objectives}

With the recent evolution of synthetic cathinones there is minimal research conducted in the forensic field. Most studies focus on understanding the toxicological aspects, consumer use, and ability to purchase of these drugs. Yet with the new findings there is little to no application that can be applied to diminishing the overwhelming amount of bath salts that evade law officials and are smuggled into this country. One major reason is that there are limited new findings that directly address the issue. Scientifically providing solutions can begin with conducting research. To date there is no information or findings pertaining to the VOCs released from bath salts. Furthermore, there is very little to no information pertaining to the optimal methods which can be utilized to gain further information on the characteristics of various bath salts; outside the realm of basic toxicological and pharmacology information. Thus the objective of the dissertation research was to determine the optimum extraction method used to capture qualitatively and quantitatively the VOCs released from several different synthetic cathinones. Ultimately the odor profiles created were assessed to determine the active odorants canine detection teams could use to successfully detect these drugs. Curbing the use and ease of obtaining various amounts of bath salts, by addressing the potential lack of detection in the currently employed canine teams to assist with drug seizures, was the end goal of the work concluded in this dissertation.

The present dissertation will present the results of laboratory and field experiments to obtain the goals. The tasks that were performed are listed below: 
1. Analytical Method Optimization for the Detection of Volatile Organic Compounds Released from Synthetic Cathinones

A. Literature review and preliminary analysis of synthetic cathinones

B. Comparison of different GC stationary column chemistries

i. BR-5ms (Non-polar)

ii. Sol-gel Wax (Polar)

C. Development of an optimal Gas-Chromatography method

D. Development of an optimal complimentary Chemical Ionization Mass Spectrometry method

2. HS-SPME Method Optimization for the Detection of Volatile Organic Compounds released from Synthetic Cathinones

A. Parameter Optimization to enhance the extraction of VOCs released from synthetic cathinones

3. Assessment of the Volatile Organic Compounds Released from Synthetic Cathinones

4. Imprinting Canines to Detect Illicit Bath Salts

A. Assessment of currently certified detection teams to detect synthetic cathinones

B. Imprinting canines to detect synthetic cathinones 

C. Imprinting validation of canine teams

5. Isolation of the Volatile Organic Compound(s) in Synthetic Cathinones Detected by Certified Canines for the Development of Controlled Odor Mimic Permeation Systems
A. Development of the odor introduction system
B. Introduction of canines to single compounds to identify the active odorant
C. Introduction of canines to binary mixtures to identify the active odorant

6. Optimization of the Controlled Odor Mimic Permeation System for Synthetic Cathinones
A. Selection of the ideal absorbent material to hold the odorant
B. Selection of the optimal polymer thickness to house the odorant
C. Determination of the dissipation rate and life span of the COMPS 
4 Introduction of Task: Materials and Methods

\subsection{Analytical Method Optimization for the Detection of Volatile Organic Compounds Released from Synthetic Cathinones}

Synthetic drugs are chemically modified substances possessing a similar structure to the parent compound to produce an equivalent or more physiologically potent effect ${ }^{59}$. Since 2009 , the abuse of bath salts has risen within the U.S. creating a shift in legislative focus as a result of the ease at which they are manufactured, distributed, and sold ${ }^{35}$. In lieu of all regulations and emergency scheduling attempts, the rate at which bath salts are so easily produced in clandestine labs allow for a vast number of newly manufactured second, third, and fourth generation bath salts for continued abused; with the most recent wave including the cathinone derivative known as flakka or gravel $(\alpha-$ Pyrrolidinovalerophenone).

Investigative studies into understanding user preference for beta-ketone phenethylamine analogue consumption have been explored over the past few years ${ }^{105,106}$. However, these objectives primarily focused on exposing their purchase methods, the adverse biological/chemical effects, social experiments for understanding consumer preferences, preliminary identification of synthetic cathinone standards, detection of bath salts in biological specimens, cross reactivity with immunoassays, and other toxicology studies $^{37,107}$.

The few analytical studies published to date have illustrated the detection of bath salts through Gas Chromatography Mass Spectrometry (GC-MS), Liquid Chromatography Tandem Mass Spectrometry (LC-TMS), and through the use of High Resolution Mass Spectrometry ${ }^{108-110}$. Yet studies within this realm typically require the 
use of nuclear magnetic resonance spectroscopy (NMR), derivatization of a synthetic cathinone standard, and yield results as complimentary findings within a pharmacology investigation ${ }^{111,111}$. Most studies highlight the detection of bath salts and their metabolites as they naturally appear in the salvia, blood, and urine ${ }^{36}$. There are minimal studies revealing what analytical information can be obtained from the analysis of unaltered, impure street samples encountered in the field. Thus developing the proper extraction method to achieve this must begin with the creation of an optimal analytical method. A routinely used analytical tool within the scientific community is GC-MS. As a coupled analytical technique, GC-MS works by adequately selecting a column and temperature program required for the separation and detection of various analytes. Yet, one adverse effect of studying cathinones with this technique is thermal degradation readily undergone by any given sample and the detection problems caused by severe fragmentation. Although an efficient way to overcome this problem is by undergoing derivatization of the sample. Another method, which allows for detection directly from the analytical instrument without chemical derivatization, is through chemical ionization. As a soft ionization technique, CI allows the sample's molecular ion to remain intact, which can be very beneficial during analysis. Within this first task there were several objectives: investigating current research developments on synthetic cathinones and other drugs in comparison, selecting the best containing method for extracting volatiles, and selecting the proper conditions within the GC (i.e., column and temperature programing) and MS (i.e., chemical ionization reagent and parameters) instrument required to build an optimal analytical method. 


\subsubsection{Materials}

\subsubsection{Sampling of Synthetic Cathinones}

There were three different housing containers used for all synthetic cathinones sampled during testing: 8 oz. clear screw top mason jars (Publix, Miami FL), 40-mL clear screw top PTFE vials (Supleco, Bellefonte, PA) and 4-mL clear screw top vials with hole cap PTFE/Silicone septa (Supleco, Bellefonte, PA). Each Mason jar was fixed to contain a suba-seal silicone rubber septum (Sigma-Aldrich, St. Louis, MO). The SPME fibers used during this task included $75 \mu \mathrm{m}$ Carboxen/Polydimethylsiloxane (CAR/PDMS), 65 $\mu \mathrm{m}$ Polydimethylsiloxane/Divnylbenzene (PDMS/DVB), 50/30 $\mu \mathrm{m}$ Divnylbenzene/Carboxen/Polydimethylsiloxane (DVB/CAR/PDMS), and $85 \mu \mathrm{m}$ Polyacrylate (PA). These fibers were purchased from Sigma Aldrich (St. Louis, MO). The heating apparatus employed during this task was an Isotemp digital and analog dry bath incubator (Fisher Scientific, Hampton, NH). The first bath salt case used in this task was case number 1648297 containing suspected Methylone. The case was retrieved from the Miami Dade Police Department crime lab (Doral, FL).

\subsubsection{Reference Standards and Material}

The standards used in this task, found in table 7, were 3,4Methylenedioxyprovalerone $\mathrm{HCl}$ (MPDV), Methylone $\mathrm{HCl}$, 1,3-Benzodioxole, 4-Bromo1,2-(methylenedioxy)benzene, and 2,4-bis (1,1-dimethylethyl)-phenol. All synthetic cathinone standards were purchased from Cerilliant (Round Rock, TX) and the remaining standards were purchased from Sigma-Aldrich (St. Louis, MO). All solvents (methylene chloride and methanol) used were purchased from Sigma-Aldrich (St. Louis, MO). All 
mixtures were housed in clear 2-mL ABC vials with PTFE/Silcone lined caps and 22-mL Screw top solid cap amber vials with PTFE liners (Sepleco, Bellefonte, PA).

Table 7. Standards Purchased from Vendors

\begin{tabular}{|c|c|c|c|}
\hline Compound & CAS No. & Vendor & Lot/Batch No. \\
\hline MDPV & $24622-62-6$ & Cerilliant & M-146 \\
\hline Methylone & $186028-80-8$ & Cerilliant & M-140 \\
\hline $\begin{array}{c}\text { 1,3-Benzodioxole } \\
\text { 4-Bromo-1,2- } \\
\text { (methylenedioxy)benzene }\end{array}$ & $274-09-9$ & Sigma-Aldrich & 159166 \\
\hline $\begin{array}{c}\text { 2,4-bis (1,1- } \\
\text { dimethylethyl)-phenol }\end{array}$ & $2635-13-4$ & Sigma-Aldrich & 288314 \\
\hline
\end{tabular}

\subsubsection{GC Column Chemistries}

Two analytical instruments were used during this task. The first GC was a Bruker Scion 456 gas chromatograph coupled to a single quadrupole mass spectrometer (Billerica, MA). A Varian CP-3800 gas chromatograph coupled to an ion trap Saturn 2000 mass spectrometer (Walnut Creek, CA) was also used. For direct automatic injection the Bruker was equipped with a CP-8400 autosampler (Varian Inc., Walnut Creek, CA). The Varian was equipped with a Combipal autosampler (Agilent Technologies, Santa Clara, CA).

The two column types used for analysis were: Bruker BR-5ms FS (non-polar) column $15 \mathrm{~m}$ in length, internal diameter (ID) of $0.25 \mathrm{~mm}$, and film thickness of $0.25 \mu \mathrm{m}$; Varian SolGel-Wax (polar) column with a length of $30 \mathrm{~m}$, internal diameter of $0.25 \mathrm{~mm}$, and film thickness of $0.25 \mu \mathrm{m}$. 


\subsubsection{Chemical Ionization Reagents}

Chemical Ionization was achieved using the Saturn 2000 Ion Trap mass spectrometer (Walnut Creek, CA). The reagents used during chemical ionization are listed in table 8.

Table 8. Chemical Reagents used to determine the Optimal Chemical Ionization Method

\begin{tabular}{|c|c|c|c|}
\hline Reagent & CAS No. & Vendor & Lot/Batch No. \\
\hline Acetonitrile & $75-05-8$ & Fisher Chemical & 158950 \\
\hline $\begin{array}{c}\text { Ammonium } \\
\text { Hydroxide }\end{array}$ & $1336-21-6$ & Sigma Aldrich & 11496 \\
\hline Methanol & $67-56-1$ & Fisher Chemical & 154246 \\
\hline
\end{tabular}

\subsubsection{Methodology}

\subsubsection{Development of Sampling Parameter and Selection of Standard Compound Testing Pool}

The subtask was used to determine the appropriate containing system to sample all encountered cases. Initially each jar's screw top was manufactured to allow SPME fibers to sample its containments. The center of the top was pierced with a screw driver and a silicone septum was inserted into the opening to create a puncture site for the SPME fiber. The 8 oz. clear screw top mason jars (Publix, Miami FL) were washed with an alkaline liquid detergent dilution (Contrex Al, King of Prussia, PA), rinsed with acetone, and placed in a Fisher scientific isotemp conventional oven for 24 hours to bake. The sterilized jars were transported to the crime lab and used to house the sample. Case 1648297, containing Methylone, was identified and noted for the consistency, color, odor, and any physical traits it possessed and $10 \pm 0.1$ grams of the sample was weighed out and placed in each jar. The jars were sealed and stored in an isolated evidence locker and left to equilibrate for 24 hours. After 24 hours, the varying fiber chemistries and 
polarities in table 9 were pierced through the spectra, lowered to a setting of 3, exposed for an hour, and then analyzed on the GC-MS.

After testing the Mason jar, 40-mL vials were cleaned with the same process as the mason jars and baked for 24 hours. Approximately $2.5 \pm 0.2$ grams of the methylone case was placed in the vials and equilibration commenced for 24 hours. Thereafter the fibers were exposed at a depth of 1 for an hour and analyzed with the GC method. The same procedure was followed for 4-mL vials, excluding the washing phase. The amount of methylone placed in each vial was $1 \pm 0.02$ grams. The sampling containers were compared to determine which offered an enhanced depiction of the volatiles within the headspace. A list was compiled in efforts to grasp an understanding on the types of compounds prematurely identifiable. Stock solutions of $10,000 \mathrm{ppm}$ were created with a methylene chloride solvent (Fischer Chemical, Fair Lawn, NJ) and stored in 22-mL amber screw solid top with PTFE liner vials (Supleco, Bellefonte, PA). Methylone and MDPV stock solutions of 1,000 ppm were created with methanol and stored in clear 2$\mathrm{mL}$ ABC vials with PTFE/Silicone lined caps (Supleco, Bellefonte, PA). The stock solutions were diluted down to $50 \mathrm{ppm}$ using methylene chloride and ran for confirmation. From this list a small number of compounds were chosen to operate as the active standard pool for GC-MS optimization. 
Table 9. Six Fiber Chemistries used to determine the Potential Number of Compounds Present to Develop a Standard Pool

\begin{tabular}{|c|} 
Fiber Chemistry \\
\hline $75 \mu \mathrm{m}$ Carboxen/Polydimethylsiloxane CAR/PDMS \\
\hline $60 \mu \mathrm{m}$ Carboxwax/Templated Resin CW/TPR \\
\hline $65 \mu \mathrm{m}$ Polydimethylsiloxane/Divnylbenzene PDMS/DVB \\
\hline $7 \mu \mathrm{m}$ Polydimethylsiloxane PDMS \\
\hline $50 / 30 \mu \mathrm{m}$ Divnylbenzene/Carboxen/Polydimethylsiloxane DVB/CAR/PDMS \\
\hline $85 \mu \mathrm{m}$ Polyacrylate PA \\
\hline
\end{tabular}

\subsubsection{Development of an Optimal GC Method and Selection of a GC Column Chemistry}

The appropriate selection of a GC column and method began with employing the non-polar Bruker BR-5ms column. The parameters optimized for enhanced resolution and separation primarily focused on temperature programing and a split versus split-less injection port. The initial extractions performed utilized six SPME fibers which were ran on the BR-5ms column. From the standard method created the ramping and injection splits were modified and compared to determine the most suitable method. The optimal method was then applied to a Solgel-Wax column and compared to the BR-5ms column chemistry. The column performance were assessed on the basis of on peak resolution and separation, in addition to the number compounds detected. Thereafter, the optimum column and method for gas chromatography was selected. 


\subsubsection{Development of a Chemical Ionization Mass Spectrometry Method}

Determination of the optimal CI method was chosen using the compounds identified during the development of the GC method selection; including two heavily encountered synthetic cathinones (MDPV and Methylone). Stock solutions of 1,3Benzodioxole, 4-Bromo-1,2-(methylenedioxy)benzene, 2,4-bis(1,1-dimethylethyl)phenol, Methylone, and MDPV were used to create a $50 \mathrm{ppm}$ mixture of all standards placed inside 2-ml clear ABC vials. The vials remained refrigerated until the solution was analyzed on the Saturn 2000 Ion Trap mass spectrometer. The chemical reagents tested were Acetonitrile, Ammonia (produced from Ammonium hydroxide), and Methanol. The desired liquid was pipetted into the instrument's CI bulb until approximately halfway full. The bulb and reagent liquid were allowed to expel vapors into the instrument for fifteen minutes. Thereafter a system check was conducted to ensure that the appropriate reagent volume was entering the system to perform CI. The parameters modified were chemical ionization storage level, ejection amplitude, background mass, maximum ionization time, and maximum reaction time. Each parameter was modified independently and the methods corresponding to the parameter were compared to determine which resulted in an increase in the spectral information and sensitivity obtained. Each parameter was continuously optimized until the reagents' chemical method was optimally created. The CI methods created for each reagent were compared to select the best working reagent and method required for successful chemical ionization. 


\subsection{HS-SPME Method Optimization for the Detection of Volatile Organic Compounds Released from Synthetic Cathinones}

Throughout the years, several extraction methods have been employed to analyze a variety of narcotics. To this day, many extraction methods include liquid-liquid extraction and solid phase extraction to separate the desire analyte from any matrix. In 1990, a non-exhaustive extraction technique called SPME was developed by Pawlisyzn ${ }^{83}$. Initially developed as a more selective and specific extraction technique for analytes found in various samples (fire debris, alcohol, and liquid samples), SPME has proven beneficial for analysis conducted in the field and in various time frames ${ }^{12,112}$. Since its development SPME has supported profound discoveries in understanding and developing knowledge as it pertains to certain materials (i.e. narcotics). Solid-phase Microextraction has been used detect odor signatures in cocaine, ecstasy tablets, methamphetamine, heroin, and traces of drug or isolated compounds on US currency. The extraction method has obtained swift and sensitive results pertaining to VOC profiles of trace amounts, identification of known and unknown analytes, and has allowed for efficient field application to be achieved.

Thus far the analytical extraction methods applied to synthetic cathinones have focused mostly on solid phase extraction, Direct Analysis in Real Time (DART), and liquid extractions. The extractions are typically applied to hair analysis or separation and detection of metabolites in urine samples. Although some studies utilize SPME, their use involves the direct immersion of the fiber into a liquid sample. Headspace-solid phase microextraction (HS-SPME) has never been employed to analyze any illicit sample of bath salts without prior sample preparation. 
With the overwhelming manipulation and vast creation of synthetic cathinones and various implementation created by legislation to minimize these "legal highs" there is a large need to gather information on all aspects of these drugs. Thus far studies have begun to shed light on the metabolites produced through kinetics and the detection of the drug within biological specimens, and many other pharmacological aspects of bath salts. Analyzing synthetic cathinones through SPME GC-MS can present scientists with a different perspective of understanding the fluidity of synthetic cathinones within the community; a perspective, as discussed later, which can be directly applied to curving such an influx. The goal was to develop the extraction parameters required for successful headspace extraction of synthetic cathinones.

\subsubsection{Materials}

\subsubsection{Sampling of Cathinone Derivatives}

All synthetic cathinones tested were supplied by local Miami-Dade county crime labs (Miami, FL, USA). The SPME fibers tested are discussed in section 4.1. For housing of the SPME fibers a small pelican case was used from Pelican Products (Torrance, CA). All standards purchased were obtained from Fisher Scientific (Hampton, NH), Sigma Aldrich (St. Louis, MO), and Cerilliant (Round Rock, TX). For analysis and calibration 4-mL clear screw top vials with hole cap PTFE/Silicone septa and clear 2-mL ABC vials with PTFE/Silcone lined caps (Supleco, Bellefonte, PA) were used, respectively. 


\subsubsection{Methodology}

\subsubsection{Developing an Optimal HS-SPME method for VOC(s) Collection from Synthetic Cathinones}

The subtask involved calculating the different parameters within SPME to promote the binding affinity between the chosen fiber and the volatile organic compounds within the headspace. The GC-MS instrument used for analysis was the Varian GC CP-3800 coupled to a Saturn 2000 Ion Trap Mass Spectrometer; operated in electron ionization mode (Walnut Creek, CA, USA). The column used during optimization was a SolGelWax chemistry (refer to section 4.1).

Headspace optimization was performed by observing the effects the equilibrium, extraction time, and application of heat had on the release of volatile compounds into the surrounding headspace. Fiber selection was performed first using a 4-mL vial containing $1 \pm 0.02$ grams of methylone (equilibrium and extraction for 1 hour). The fibers were assessed using the number of compounds detected at room temperature and the reproducibility of the results. From the compounds identified a standard testing pool was created and used for the headspace extraction optimization.

Following fiber selection four additional parameters were optimized: equilibrium, extraction, heat application, and desorption time. During each parameter optimization, the base setting included a standard mixture of $50 \mathrm{ppm}$ (diluted in methylene chloride) housed in a 4-mL vial, extracted at room temperature. When applying heat, the vials were placed in their appropriate slot and remained stationary until the end of the extraction period. Each parameter was assessed by detection sensitivity and reproducibility. Once a 
factor was optimally chosen the base setting was modified to include the newly developed parameter before assessing the next. Each parameter underwent experimentation until an optimum extraction method was developed.

\subsection{Assess the Volatile Organic Compounds Released from Synthetic Cathinones}

Volatile Organic Compound (VOC) detection via SPME has proven quite beneficial within the science community. To date, VOCs have been used to gauge city and environmental pollutions, determine the emission of polymer materials in buildings, and associated with post mortem to characterize the volatiles pertaining to each decomposing phase. Volatile Organic Compounds extracted have also been useful in characterizing the odor profiles associated with narcotics, explosives, agriculture, and human scent.

With the recent distribution and creation influx of synthetic cathinones, the United States Drug Enforcement Administration (DEA) has been working to diminish their rise, while gathering and providing adequate information, including the number of confiscations, overdoses, deaths, etc., to organizations that are available to the public (i.e., poison control centers) $)^{34,113}$.

Collective headspace extraction of synthetic cathinones analyzed with GC-MS is yet another tool to provide further information into the chemical makeup of these everchanging analogues. With complete optimization of the ideal parameters required, successful extraction of all volatiles detected from bath salts can be achieved. Within this task the parameters determined in the two previous sections where used to analyze a variety of cases throughout south Florida. 


\subsubsection{Materials}

The color reagents used were cobalt, thiocyanate, and a marquis reagent all provided from the crime labs and created in their standard method. Color testing was performed in a round multi-well off-white porcelain spot plate (Sigma Aldrich, St. Louis, MO). The vials used to house all drugs were 4-mL clear screw top vials, hole caps with PFTE/Silicone septum and 2-mL ABC clear vials with PTFE/Silicone lined caps (Supleco, Bellefonte, PA). The heating device used to maintain the selected temperature was an Isotemp digital and analog dry bath incubator (Fisher Scientific, Hampton, NH). The $65 \mu \mathrm{m}$ PDMS/DVB fiber used was purchased from Sigma Aldrich (St. Louis, MO).

The GC-MS used for analysis was Varian CP-3800 gas chromatograph coupled to an ion trap Saturn 2000 mass spectrometer (Walnut Creek, CA); equipped with a SolGel-Wax (polar) column with a length of $30 \mathrm{~m}$, internal diameter of $0.25 \mathrm{~mm}$, and film thickness of $0.25 \mu \mathrm{m}$. The Varian was also employed with a Combipal autosampler (Agilent Technologies, Santa Clara, CA).

\subsubsection{Methodology}

Each assigned case was initially examined for packaging, markings, coloration, and consistency. The case was weighed on the crime lab's analytical scale to determine the amount of drug present. The packages were opened along the seams and the contents were tested following each crime lab's Standard Operating Procedure (SOP). The guidelines for the SOP followed that each case underwent presumptive testing in the form of color testing or RAMAN spectra. A multi-well color plate was used and each reagent was spotted on the well twice. The first row acted as the blank and the second row was 
used for sample introduction. A small quantity of the drug (approximately 0.001 grams) was taken out with a clean spatula and placed into each well to react with the reagent. The color produced gave a suggestion on the type of bath salt present in each case. Approximately 0.01 grams of the sample was placed in a $2-\mathrm{mL}$ vial and diluted with a methanol solvent containing the internal standard (code name PBSE). Another 2-mL vial, which served as the blank, was filled with the methanol solvent and internal standard. The vials were run on the GC-MS within the crime lab using their standard method for the identification of any analogue present alongside the internal standard. The case's chromatogram and spectrum were compared to the standard of the parent drug suspected and were used to confirm the presumptive test results.

The isotemp apparatus was set to $50^{\circ} \mathrm{C}$ and allowed to reach temperature while sample preparation began. The contents of the case were placed in a weighing boat (Fisher Scientific, Hampton, NH) for easy access. Each 4-mL vial (7 in total) used was pre-weighed and tared on the analytical scale. All large and rock-like drug components were folded into a weighing paper and crushed with a pestle so they would fit inside the 4-mL vial. Each vial was filled with $1 \pm 0.02$ grams of the synthetic cathinone. A blank 4$\mathrm{mL}$ vial was exposed to the surrounding atmosphere to collect all volatiles which may erroneously contribute to the VOC profile. The vials were closed tightly and placed within the istotemp's slot for the equilibration process.

The equilibration time was 15 minutes. The PDMS/DVB fibers were used to pierce the septum of each vial at an indication level just below 1 . The fibers were adjusted to a depth of 1 and exposed for 4 hours to complete the extraction process. After the four 
hours, the SPME needles were retracted back into the shaft and the heating source was immediately turned off. The fibers were taken out of their respective vial and capped with white sealing caps (Sigma Aldrich, St. Louis, MO) to protect from any damage during transport. The fibers were placed inside SPME fiber holding boxes and then inside an 1170 pelican case (Pelican Products, Torrance, CA). The cases were kept at cooled temperatures as they were transported back to the research facility (Florida International University, Miami, FL) and they were run on the GC-MS. Three fibers were run on the optimized CI method and the remaining fibers were run on the optimized EI method for detection and determination of all VOCs identified from the synthetic cathinones. Afterwards, the findings were analyzed and odor profiles were developed for each case.

\subsection{Imprinting of Canines to Detect Illicit Bath Salts}

The widespread popularity of bath salts is largely beause of their availability to consumers. Studies have shown that simply by Google searches of names like "bath salts" or "ivory wave", consumers are brought to secure websites for retail or wholesale of various types ${ }^{106}$. Websites routinely advertise bath salts as legal highs, where encryption is implemented for consumer safety, "buy one get one" specials, expedited shipping, and many more suggestive appeals at the legitimization of these narcotics ${ }^{35,38}$. Although regulations have been placed to halt incoming traffic of these drugs, a large percentage still is available throughout many neighborhoods as there is a lack of ground efforts to combat the outbreaks.

Overall, understanding the chemical makeup can be beneficial in field application for the detection of illegal synthetic cathinones and the new "legal" generational analogues; 
which have been carelessly purchased. Field detection offers a solution to the overwhelming problem with the increasing distribution influx of these drugs into the United States that go undetected by standard procedures currently employed.

Although there are a variety of detection teams which can detect a multitude of drugs, there have been concerns expressed to the capabilities of detection canines to actively detect bath salt. In this task, there was a baseline established as to whether the canine teams currently certified for narcotic detection can and will alert to various samples of these drugs; based on the narcotics they are already trained to detect. This task focused on testing whether canines are currently equipped to detect this synthetic drug.

\subsubsection{Materials}

During the testing and imprinting stages of the canine trials all synthetic cathinone cases were provided by the Miami Dade Police Department (Doral, FL) and Palm Beach Sheriff Office (PBSO, West Palm Beach, FL). The first canine trail performed involved the methylone case previously mentioned in section 4.1, a new ethylone case (004260) provided by the crime lab, and MDMA and marijuana (drug provided by the Miami Dade Police Department Canine team). The canine trials thereafter involved four bath salt cases: 17-426, 14-1856, 15-02913, and 14-2973. Each case consisted of either Ethylone or $\alpha$-PVP and the headspace had been collected for profiling. The positive controls used were provided by the PBSO canine team and included heroin and methamphetamine. The location of the Miami-Dade canine trial was on site at the Miami Dade Police Department crime lab (Doral, FL) in the cafeteria. The additional testing was conducted at Palm Beach Central High School (West Palm Beach, FL) using the hallway lockers. The 
imprinting process was performed at the Palm Beach Sheriff Office Canine Training

Facility (Palm Beach, FL). The containers used during the imprinting phase were K-9 BSD-2 HDPE Kit purchased from EliteK9 (Boaz, KY).

\subsubsection{Methodology}

\subsubsection{Initial Detection of Bath Salts}

The set up for both canine trials conducted in Miami-Dade and Palm Beach County is shown in figures 14 and 15. For the Miami-Dade trial the allotted space used was the lab's cafeteria which provided a connecting outside ramp for easy access. The section used for testing was closed off and controlled by the agency. As depicted in the diagram the hides consisted of the methylone and ethylone case for testing, the positive control, and a blanked system. The blank system did not contain any odor which would cause an alert by the canine. The positive control and the cathinone cases were located at the furthest possible distance. Each case and control were placed in pre-washed metal boxes provided by the canine teams, used during training. The hides were allotted thirty minutes at a minimum for a headspace to accumulate. The guidelines used for the setup and testing followed SWGDOG recommendations. There were five canine teams in the experiment; which was conducted single blind. The handlers were asked to come in and search in the room in the manner in which they would typically conduct a search. The handlers were informed that if their dog actively alerted, showed interest, and did not give any noticeable change in behavior to vocalize this as the responses were monitored. The canine handlers were also informed that if they K-9 correctly alerted to the positive control then the reward could be given. However, they were encouraged to not reward 
their canines for alerting to a case which contained any bath salt; instead to positively pull them off the hide and move forward. The canine trial in Palm Beach County was set up in the manner depicted in figure 15.

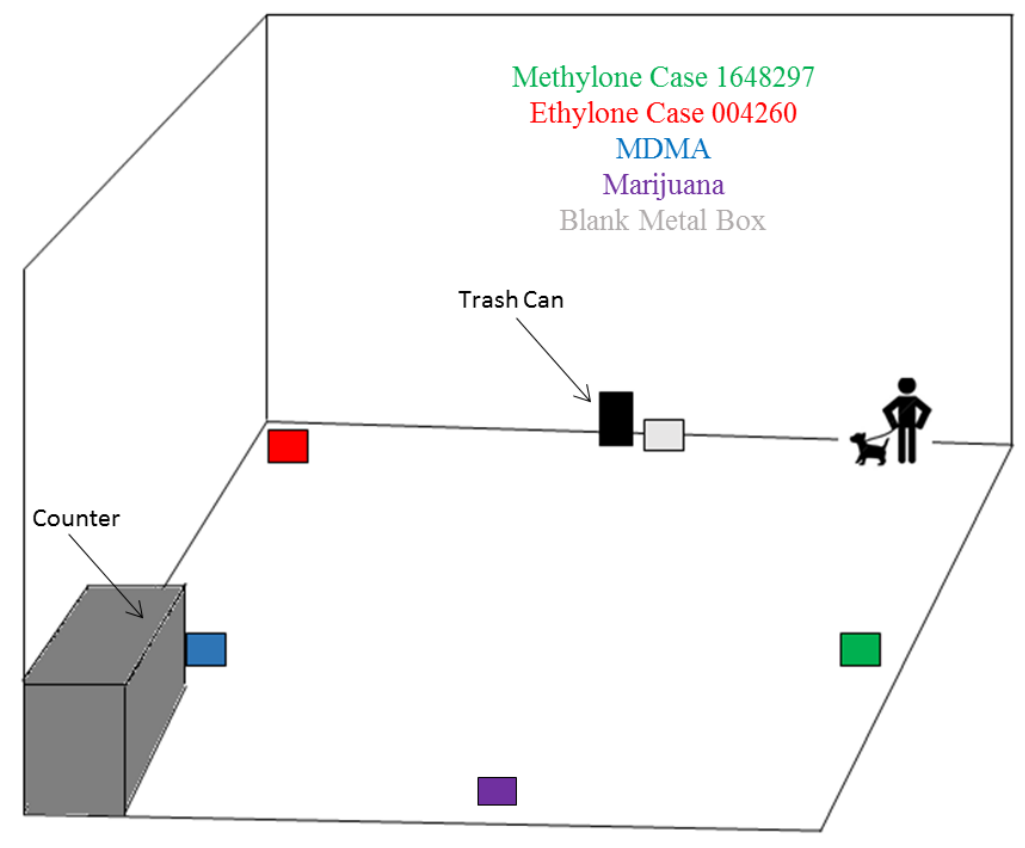

Figure 14. The Schematic of the Miami-Dade Canine Trial

Each case was placed in a separate locker corridor. Only the bottom half of the lockers were used to optimize canine odor interaction as the trainer had previously conducted K-9 work in this manner. The cases were supplied by PBSO through the required standard for drug retrieval for canine detection work. Each case arrived in heat seal non-permeable bags and was opened to retrieve the inner bags. Each case contained approximately 10 grams of the bath salt. All designated lockers were opened and with sterile gloves, the inner bags were taken out and placed in the seam of the locker. 
Although the doors were closed, the lockers remained unlocked as the engraved dial remained unfastened. The drugs were given thirty minutes to accumulate efficient headspace, while the temperature and humidity settings were recorded. Thereafter each canine handler was given the same instructions as the Miami-Dade canine teams and the trial began. The alert, interest, and non-alerts observed by the handler were noted for record. 

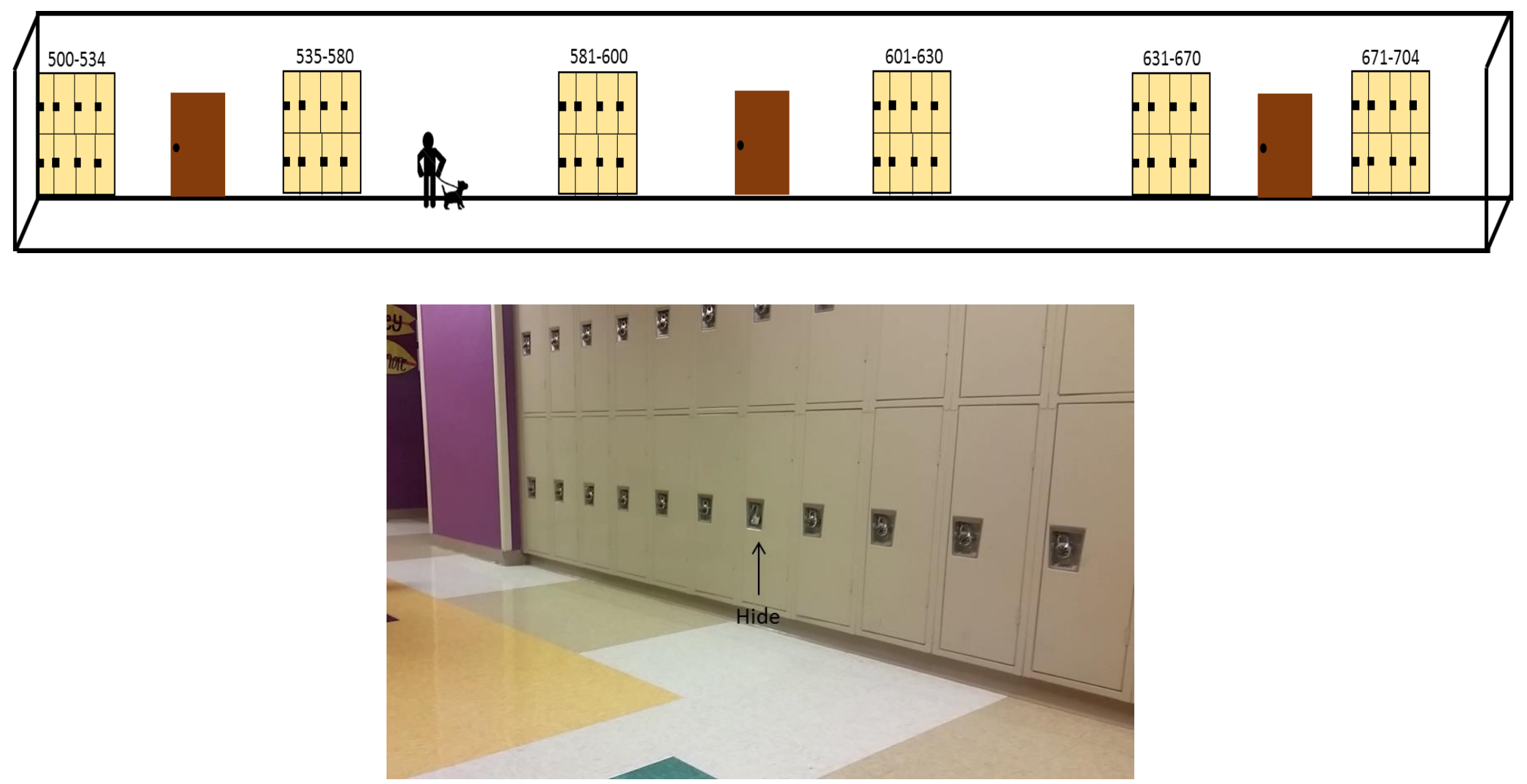

Figure 15. Schematic of Palm Beach Canine Trial 


\subsubsection{Imprinting on Bath Salts}

The imprinting process is where learned behavior is applied to teach the canines how to recognize a new odor as something that should result in an alert. An alert is any passive behavior such as sitting or lying down to indicate the presence of a given substance. During this process the canines had to be taught to recognize the order of the new drug (bath salts). The canine teams were divided into separate groups. Half of the class was imprinted on the ethylone cases and the remaining was imprinted on the $\alpha$-PVP cases.

The first stage of imprinting was introducing the canine to the odor. Each case was placed inside of a cleaned mason jar and screwed into the open slot within a Behavior Shaping Device (K-9 BSD-2 kit; popper box). Known as the popper box, these training devices are equipped with a launcher that house a reward and eject it for positive reinforcement. Clean hand towels were rolled, taped in place, and positioned in the shooter hole. One box contained the odor and the remaining four boxes were blank. The odor accumulated for 30 minutes and the first dog was brought in. During the first test, the canine was walked along the boxes and brought to the odorant box where it was allowed to engage in the sniffing processing. At this point the shooter ejected the towel out and the canine was allowed to grab the reward. This phase was repeated at least three times per session ( 3 sessions in a week). The second phase was undergone by developing the searching pattern. The canine was brought in and given the command to search, if the canine passed the odorant box, they were corrected, swung back around, and reintroduced to the box. When the dog remained stationary at the box, the shooter ejected the towel 
and the canine collected its reward. This process was repeated for several weeks until the dog was able to sit or alert its handler to the presence of the drug.

The third phase incorporated distractors. The odorant box with the bath salt was present. The blank mason jar on the other boxes was filled with dog food, tennis balls, or other distractors to test the canine's alertness, sensitivity, and reproducibility of positive indications. The boxes remained idle for the first 30-40 minutes prior to the trial. The canines were given the active command to search. Corrections were given where needed to be, until satisfactory results were achieved for the trainer.

\subsubsection{Imprinting Validation of Canine Teams}

After completion of the imprinting process the canines were tested for validation. The cases were placed in unmarked boxes approximately 10 feet apart. The search included distractors, and MDMA as the positive control. Following the guidelines of SWGDOG, the canine teams conducted the search and the alerts were recorded. The canines had to receive a minimum score of $85 \%$ detection to be confirmed as successfully imprinted on the new drug. All other canine teams that score lower than $85 \%$ were asked to reinforce the imprinting process and perform the validation test after another week. The successfully imprinted canines were used to determine the active odorant for the synthetic cathinones. 


\subsection{Isolate the Volatile Oragnic Compound(s) in Synthetic Cathinones Detectable by Ceritified Canines}

The policing community has always noticed the advantages to using a canine's olfactory sensors for detection work. Understanding the canine's keen sense of smell and using it in the most efficient manner; has been a role within the scientific community for years. Various studies have showcased that by isolating the compounds responsible for the odor of any illicit material, it is possible to determine the odorant responsible for a canine's alert.

This task took the approach of breaking down the headspace (odor) of the bath salt into singular component. At which point exposing the canines to the odor in a controlled manner to determine the odorants actively responsible for a canine's receptive response of the drug's presence.

\subsubsection{Materials}

All solutions created were diluted with Methylene Chloride and stored within 40$\mathrm{mL}$ clear screw top hole caps vials with PTFE/Silicone septum (Supleco, Bellefonte, PA). Compounds were introduced to the canines after developing an odor bag. The developing bags used were 3 x 3inch 2 mil Low Density Polyethylene (LDPE) bags purchased from Uline (Pleasant Prairie, WI). The interior of the bag contained a $2 \times 2$ inch 8PLY sterile surgical sponge gauze manufactured by Independent Medical Co-op (Catalog No. 6208IMC, Daytona Beach, FL). All compounds used during this task were purchased from Sigma-Aldrich and Fisher Science. The bag closure was completed by using a Midwest 
Pacific Impulse heat sealer from Packco Inc. (Rocky Mount, MO). A standard watch glass was purchased from Fisher Science.

\subsubsection{Methodology}

\subsubsection{Developing the Organic Compound Introduction System}

The standards used during the odorant selection were received in two different states: liquid and solid. All standards received in the solid form were diluted to a 100,000 ppm stock solution using methylene chloride, stored in a $40-\mathrm{mL}$, and kept at a cooled temperature until extracted. With sterile gloves the 2 mil LPDE bags where obtained and heat sealed once along the natural crease of the bag. One surgical gauze was placed inside the bag and for all liquid standards, $10 \mu \mathrm{L}$ was pipetted into the bag and heat sealed three times. The bags were placed inside of a $40-\mathrm{mL}$ vial, labeled, and parafilmed.

In order to assure that the compounds diluted only contained the standard (excluding the solvent). There needed to be a test to determine the amount of time required for the evaporation of the solvent thus leaving the compound residue behind. For the odor bags a $10 \% \mathrm{w} / \mathrm{v}$ was used. Therefore from the stock solution created $100 \mu \mathrm{L}$ was pipetted to give a final amount of $10 \mathrm{mg}$ of the compound on the gauze. To test the length of evaporation required a clean surgical gauze was placed on a clean watch glass and both were weighed together. One hundred microliters $(\mu \mathrm{L})$ of methylene chloride was pipetted on the central area of the gauze and a second weight was recorded. The weight of the system was recorded in fifteen minute increments until the secondary weight returned to the initial. The total time was calculated to determine the length of time needed to completely evaporate methylene chloride. 


\subsubsection{Active Odorant Determination: Single Compound and Binary Mixtures}

Odor bags were created the night before every canine trial and solutions were kept in the refrigerator prior to creation. The LDPE bags were heat sealed at the bottom, contained one gauze, and were spiked with $10 \mu \mathrm{L}$ of the pure liquid compound. All $10 \%$ w/v solutions were spiked on a gauze, placed on a watch glass, and sat for the allotted time. Afterward the gauzes were placed in a separate bag and triple sealed at the top. All bags were placed in $40-\mathrm{mL}$ vials and parafilm was wrapped around the containers. The vials were placed an 1150 pelican case, along with a pair of gloves, alcohol pads, and an environmental meter (PCE Americas, Jupiter, FL).

Canine trials were conducted at two locations: Palm Beach Central High School (West Palm Beach, FL) and the Palm Beach Airport Off-site Facility (West Palm Beach FL). With trials located at the school each bag was placed in separate a locker corridor, within the seams of each locker as depicted in section 4.4. Trials conducted at the off-site facility involved cement blocks with openings that sheltered the bags from the canines (Figure 16). The blocks were located along the side and back wall of the building and were spaced 10 feet apart. Each vial was handled with gloves and the bags were removed with pre-cleaned tweezers. 


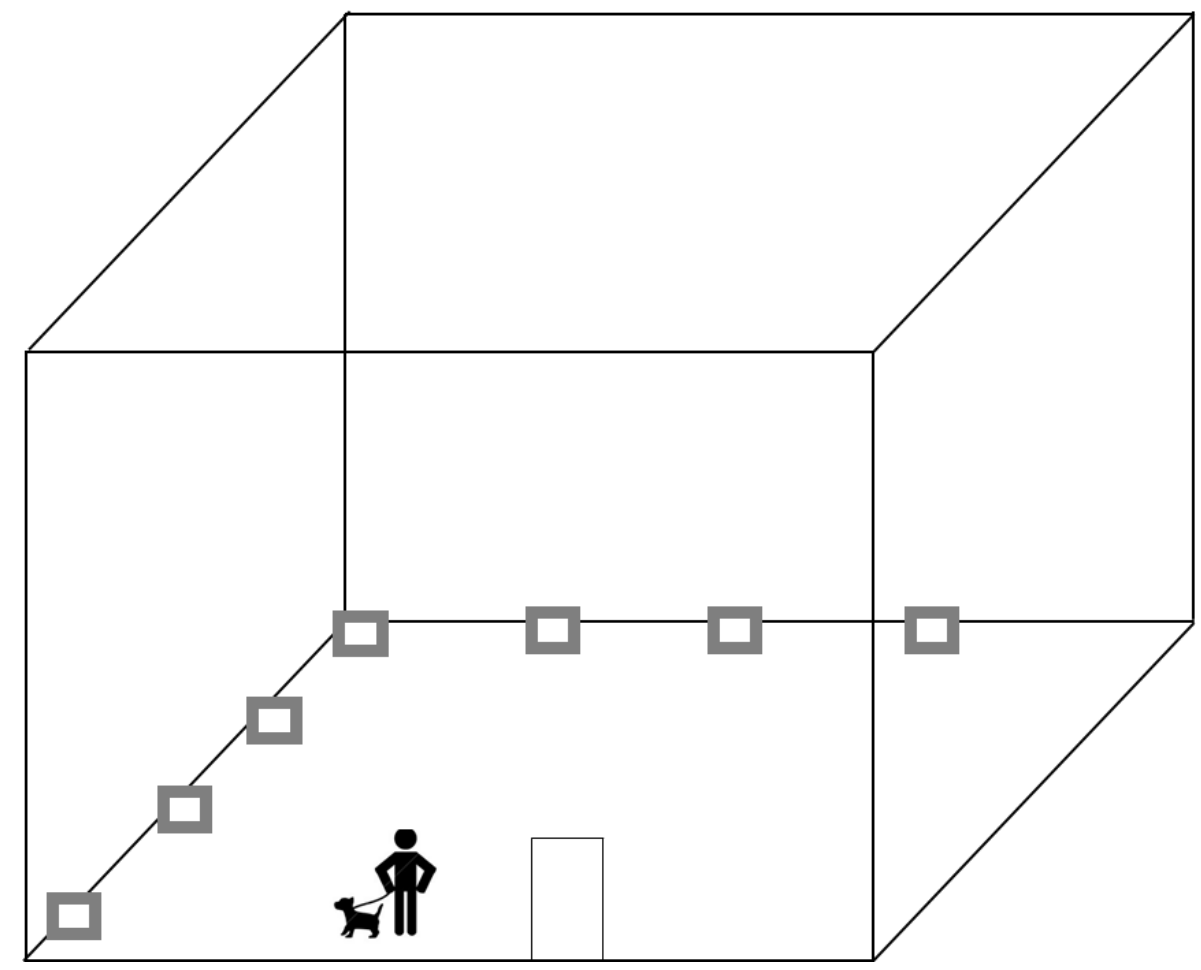

Figure 16. Schematic of the Trial Setup at the Palm Beach off-site Airport Facility

Using the guideline on the SWGDOG website, the bags were given 30 minutes for the headspace to accumulate. The canine handlers were informed that the bags contained a specific compound found in the odor profile of the bath salts. The handlers were informed that if the canines alerted to the compound they would not reward the canines but proceed through the search. The handlers were informed to let the observer know if their canine alerted, showed interest, or no indication at all. The handlers were notified if their canine had alerted to any bath salt case or a positive control at which point they were allowed to reward their canines with the same towel reward described in section 4.4. The trials were repeated multiple times and the indications were recorded. Any compound which initiated a high interest or alert was combined into ratios of each other. The odor bags were created in the same fashion as expressed above. The binary 
mixtures and singular components were also ran to determine if they canines would increase their alerting ratio when encountering the mixture.

\subsection{Optimization of the Controlled Odor Mimic Permeation System for Synthetic Cathinones}

Throughout the years there have been several cases in which a canine has accidently died from overdosing on drugs. These substances are encountered in the field and during training where the dog accidently bites on the aid. Training canines for the detection of drugs has brought concern with the exposure of such potent narcotics to smaller living organisms for the benefit of human needs. Over the years there has been a heightened need for a safe way to train canines to detect illicit materials in the field.

In the research community the detection and identification of volatile organic compounds (VOCs), often referred to as the odor, in several illicit drugs, such as cocaine, ecstasy (MDMA), and methamphetamine have been achieved by SPME/GC-MS ${ }^{82}$. Previous research identified methyl benzoate as the active odor of cocaine and is currently being used for canine training purposes. In addition, methylenedioxymethamphetamine (MDMA) and methamphetamine (MA) (structurally similar to synthetic cathinone derivatives) were found to release piperonal and benzaldehyde, respectively, which were detectable by trained narcotic detection canines. Although these compounds may not be the active ingredient in the illicit substance, ongoing research has revealed that these VOCs are reliable indicators to the presence of the active drug, and thus have been employed as a drug odor mimic for canine training. 
Controlled Odor Mimic Permeation System (COMPS) is a system designed to release the active odor of an illicit substance through a permeable bag, at a constant rate. In the past, an odor recognition study with more than 180 trained and certified explosive detection canines was conducted to assess the efficacy of six different COMPS, each containing the active odorant of an explosive. The results revealed the success of COMPS, as more than $93 \%$ of the canines that were evaluated positively alerted to the odor mimic proving its ability to be a viable substitute for canine training when the actual substance is not available. In addition, COMPS are highly advantageous as they reduce the risk of exposure to hazardous materials for both canine and handler, do not require a special license to carry or store, and produces detectable levels of the active odorant.

Within the task an optimal Synthetic Cathinone COMPS was developed for deliverance of the active odorant. The task involved developing the optimal mixture and set parameters that would increase the longevity of the COMPS for continued use throughout the years.

\subsubsection{Materials}

Throughout the course of optimization the same optimized PDMS/DVB fiber was used (Sigma Aldrich, St. Louis, MO). The items used during SPME optimization were a 40-mL clear screw top hole caps vials with PTFE/Silicone septum (Supleco, Bellefonte, PA), 10-mL screw top hole caps vials with PTFE/Silicone septum (Supleco, Bellefonte, PA), and a 2 x 2 inch 8PLY sterile surgical sponge gauze manufactured by Independent Medical Co-op (Daytona Beach, FL). All COMPS were sealed with the Midwest Pacific Impulse heat sealer from Packco Inc. (Rocky Mount, MO). All LPDE bags used for 
optimization were purchased from U-line (Pleasant Prairie, WI). The stationary vehicles used to develop the COMP was a $20 \mu \mathrm{m}$ microcrystalline Cellulose powder (Catalog No. 310697,Simga-Aldrich, St. Louis, MO) and 80-200 mesh Alumina adsorption (Lot No. 162621, Fisher Chemical, Fair Lawn, NJ). The active odorant 3,4Methylenedioxypropiophenone was purchased from Sigma-Aldrich (St. Louis, MO). All COMPS were suspended on a wire netting placed inside a pre-cleaned glass tank with the glass roof. During extraction the COMPS were placed in half pint 8 oz. mason jars (Ball, Fishers, IN) with a suba-seal silicone rubber septum (Sigma-Aldrich, St. Louis, MO).

\subsubsection{Methodology}

\subsubsection{COMPS: HS-SPME Optimization}

The SPME parameters used to develop the VOC profile for the synthetic cathinones were reevaluated to detect the odor dissipating from the COMPS. A $2 \times 2$ inch gauze was cut down to a $1 \times 1$ inch dimension and placed inside of a 40-mL vial; $100 \mathrm{ng}$ of 3,4-Methylenedioxypropiophenone was added to the vial. The vial was closed and equilibrated for 15 minutes. The PDMS/DVB fiber was exposed to the vial for an extraction time of 15 minutes. The SPME fiber was run on the same optimized GC-MS method and desorption was held for 15 minutes. The extraction was repeated with varying equilibrium times of 5, 30, and 60 minutes. Triplicates were performed and selection of the optimal equilibration time for the COMPS analysis was chosen. Extraction times were evaluated by comparing 1, 5, 15, and 30 minutes frames followed by 1 and 1.5 hours. Triplicates were performed and an optimal extraction time for COMPS was chosen. After the equilibration and extraction times were chosen the 
selection and optimization of an externally sampled internal standard, to account for environmental fluctuations encountered when optimizing the COMPS, was selected. Thereafter the extraction method used to determine the compound dissipation during COMPS development was established.

\subsubsection{COMPS Optimization}

A 1:10 ratio of compound to matrix was added to a $3 \times 3$ inch 2 Mil bag. The LDPE bag was heat sealed along the bottom cease and tripled sealed at the top. Twelve bags were created: 3 bags were created using cellulose, 3 using alumina as the matrix, 3 bags contained a gauze pad, and a blank bag for each matrix that contained no compound. The aids were weighed before the compound was added and then the initial weight was recorded. For the blank aids, the empty bags were pre-weighed and weighed after the matrices were added. As the bags were created they were immediately suspended within a clean glass tank. Once all bags were completed, the first set was extracted to determine the initial amount permeating through the bag. Each bag was weighed, extracted, and the humidity and temperature readings were recorded daily for one week. Afterwards, mass verses time was plotted to determine the permeation rate of each mixture. The matrix successful at slowing down the permeation rate while allowing for an efficient and controlled release of the active odorant was optimally selected for the COMPS mixture.

After the mixture was selected the effect of bag thickness on the permeating rate was examined. All bags were kept at a constant dimension of $3 \times 3$ inches. The varying thicknesses examined were 1.5, 2, and 4 mil LDPE bags. The COMPSs were created in the same fashion as previously stated, containing the ideal mixture of the pusedo. The 
bags were suspended in the tank and headspace was examined on the first day. The weights were monitored daily for one week. The temperature and humidity readings were tabulated daily. The most suitable thickness for the prolonged use of the COMPS was selected.

The containment study performed was used to determine the proper storage of the aid that would facilitate the equilibrium and protect the COMPS from contamination. The optimal LDPE bag was heat sealed along the seam and 10 grams of the matrix was placed inside as a blank. Three blank bags were placed in the tank suspended on the wire. Another set of blank bags were created and placed in a barrier foil Ziplock bag and suspended in the tank. All blank COMPS were headspace prior to the containment study. A $50 \mathrm{~mL}$ beaker containing 1 gram of methyl benzoate was placed at the bottom of the tank. Each blank COMPS was weighed and the headspace was extracted to detect the presence of the interfering odorant every day for one week. The results of the exposed versus and sheltered COMPS were compared to determine if the alumina bag successfully acted as a barrier.

\subsubsection{COMPS Validation}

The optimal COMPS created was given to three canine teams to be used to imprint as described in section 4.4. Once the canines had finished the imprinting process, a canine trial was conducted. The canine trial included the COMPS used to imprint and the cases of bath salts. The canines were tested to determine if the canine, although not imprinted on the raw material, had the capabilities to alert to the real street samples of bath salts encountered in the field. Successful examination of the canine's ability to 
detect these drugs within a controlled setting and within the field, when imprinted on the COMPS created for synthetic cathinones, was used as verification and validation of its accuracy.

5 Results and Discussion

\subsection{Analytical Method Optimization for the Detection of Volatile Organic Compounds Released from Synthetic Cathinones}

The goal of this task was to determine the compounds associated with the headspace extraction of synthetic cathinones, through literature and prematurely through experimental measures. The study also served to develop the ideal parameters for extraction and the analytical method for analysis.

\subsubsection{Parameter and Method Optimization}

After conducting the initial literature search, as hypothesized, there was no information pertaining to the HS-SPME extraction of bath salts. There was no published literature to suggest the type or classes of VOCs potentially identified within these drugs. As a result the foundation had to be established. The first step to getting the optimal extraction method for these drugs was determining a group of standards that could be used during the developmental stages.

Initially working with the Miami Dade Police Department, the case 1648297 was used to conduct a preliminary study. The case was suspected to contain Methylone, confirmed through the crime labs RAMAN instrument. The methylone case was wrapped 
in a brown plastic bag and contained large rock substances that had an off-white, light brown color. The case possessed a slight odor and weighed 22.5 grams.

The first study conducted with the case was to determine the type of compounds that could be extracted. The methylone case was crushed with a pestle and 10 grams were weighed out into two clean mason jars (figure 18). The mason jars were punctured and contained three septa. The mason jars were stored in the evidence locker assigned to the analyst. The following day, after the 24 hour equilibrium process had commenced, six fibers were exposed to the jars. Each fiber: $75 \mu \mathrm{m} \mathrm{CAR/PDMS,} 60 \mu \mathrm{m}$ CW/TPR, $65 \mu \mathrm{m}$ PDMS/DVB, $7 \mu \mathrm{m}$ PDMS, 50/30 $\mu \mathrm{m}$ DVB/CAR/PDMS, $85 \mu \mathrm{m}$ PA were exposed for a one hour. The fibers were capped and taken back to the research lab (Florida International University, Miami, FL).

The GC column the fibers were run on was a BR-5ms FS $15 \mathrm{~m} \times 0.25 \mathrm{~mm}$ I.D. $\mathrm{x}$ $0.25 \mu \mathrm{m}$. Listed as a non-polar column the chemistry contained $95 \%$ dimethylsiloxane arlene and 5\% diphenyl. The method used conduct the analysis is listed below:

Method A: Split less mode; Initial temperature oven was held at $40^{\circ} \mathrm{C}$ for two minutes, ramped at $10^{\circ} \mathrm{C} / \mathrm{min}$ until the temperature of $280^{\circ} \mathrm{C}$ was reached and held for five minutes.

When analyzing the chromatograms and spectra, there was no volatile organic information successfully obtained. As displayed in the figure 17, the peaks indicated in 
the frame where contributed to the fiber and column chemistry. The lack of chromatographic evidence drawn from the first extraction was the result of the large volumetric space encountered during the extraction process. In order to address this challenge the extraction process was repeated with varying containment sizes. Using a 40-mL vial and 4-mL vial, approximately 2.5 and 1 gram(s) of the methylone was placed inside each vial respectively; figure 18 .

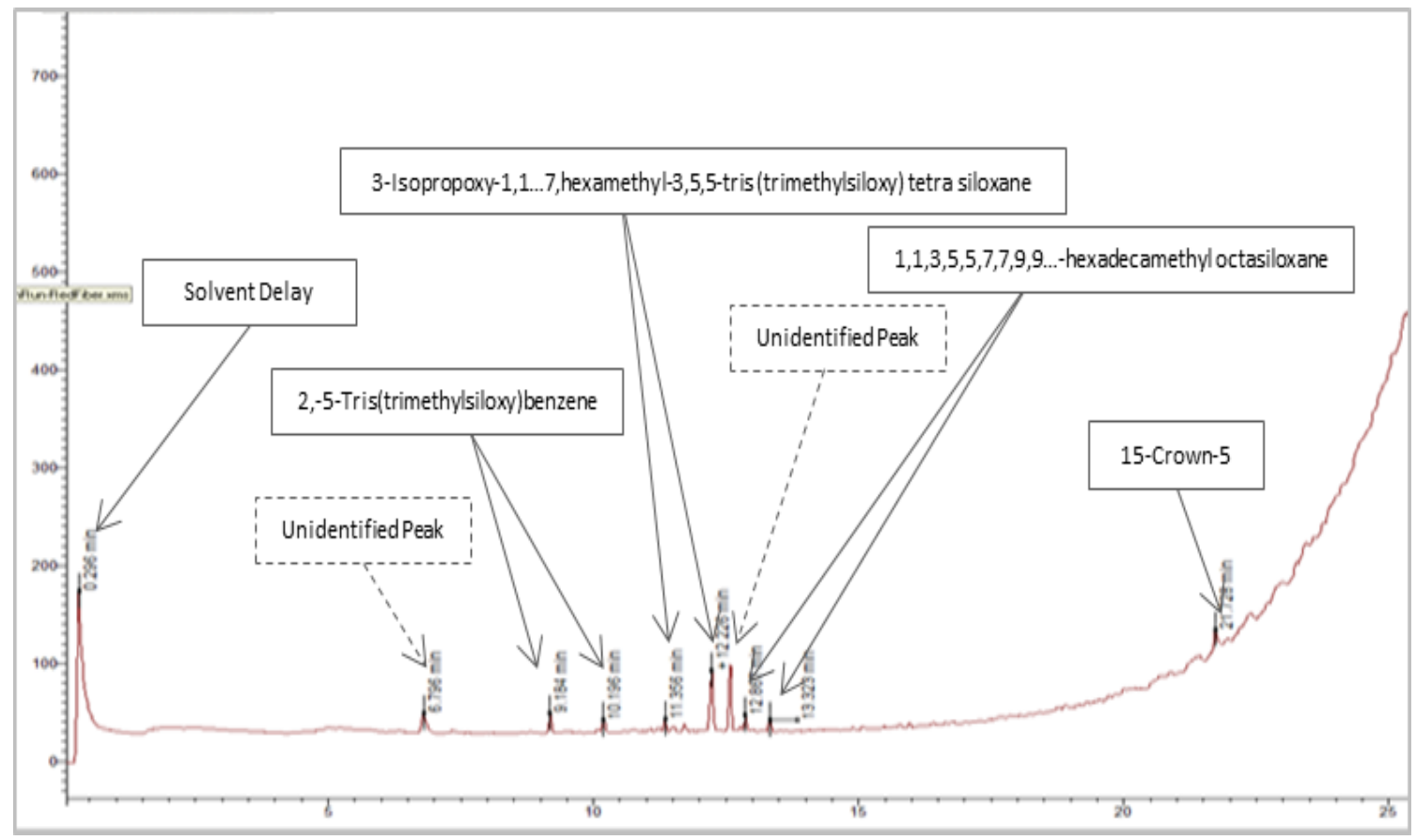

Figure 17. Reference Chromatogram for the Extraction Completed with a Mason jar 


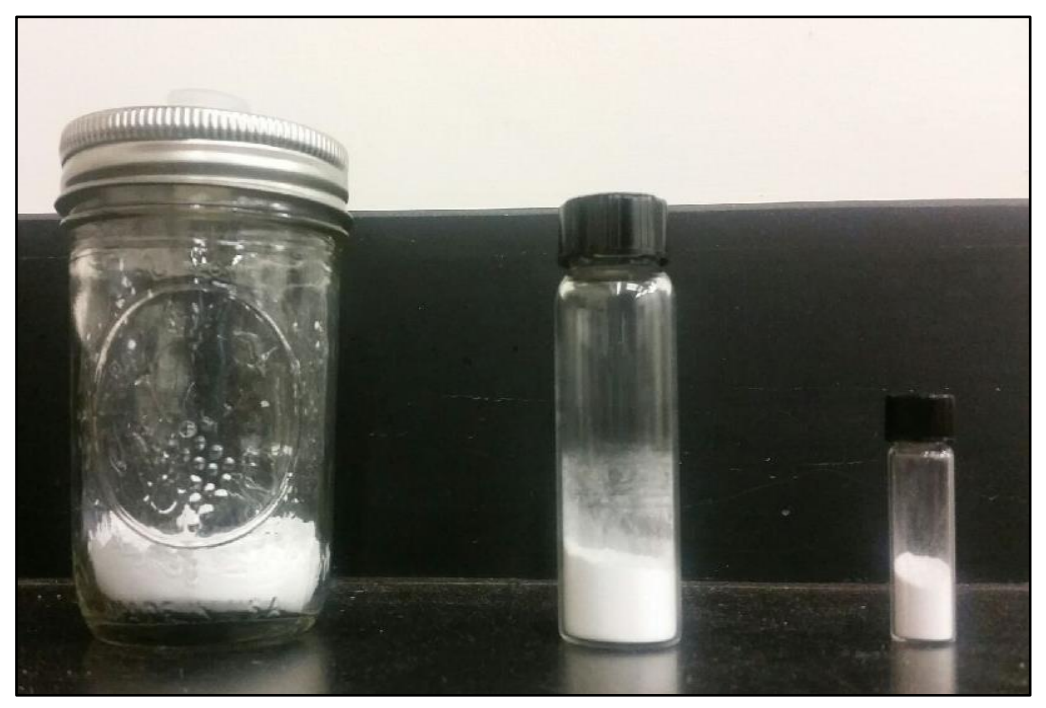

Figure 18. Representative Sampling Device Contains for the Preliminary Extraction of Methylone

The fibers were analyzed on the same column and method to determine which sampling container would yield the largest preliminary data obtainable for analysis to begin. Figure 19 shows the total peak area detected when the volume of the extraction was decreased over time. As indicated by the graph decreasing the container to 4-mL resulted in four additional compounds being identified. The RSD value percentage was slightly higher than the vials placed in the $40-\mathrm{mL}$ vial. However the $4-\mathrm{mL}$ vial used a lower quantity for extraction while resulting in a larger abundance and number of volatiles extracted and was therefore used to in future experiments. 


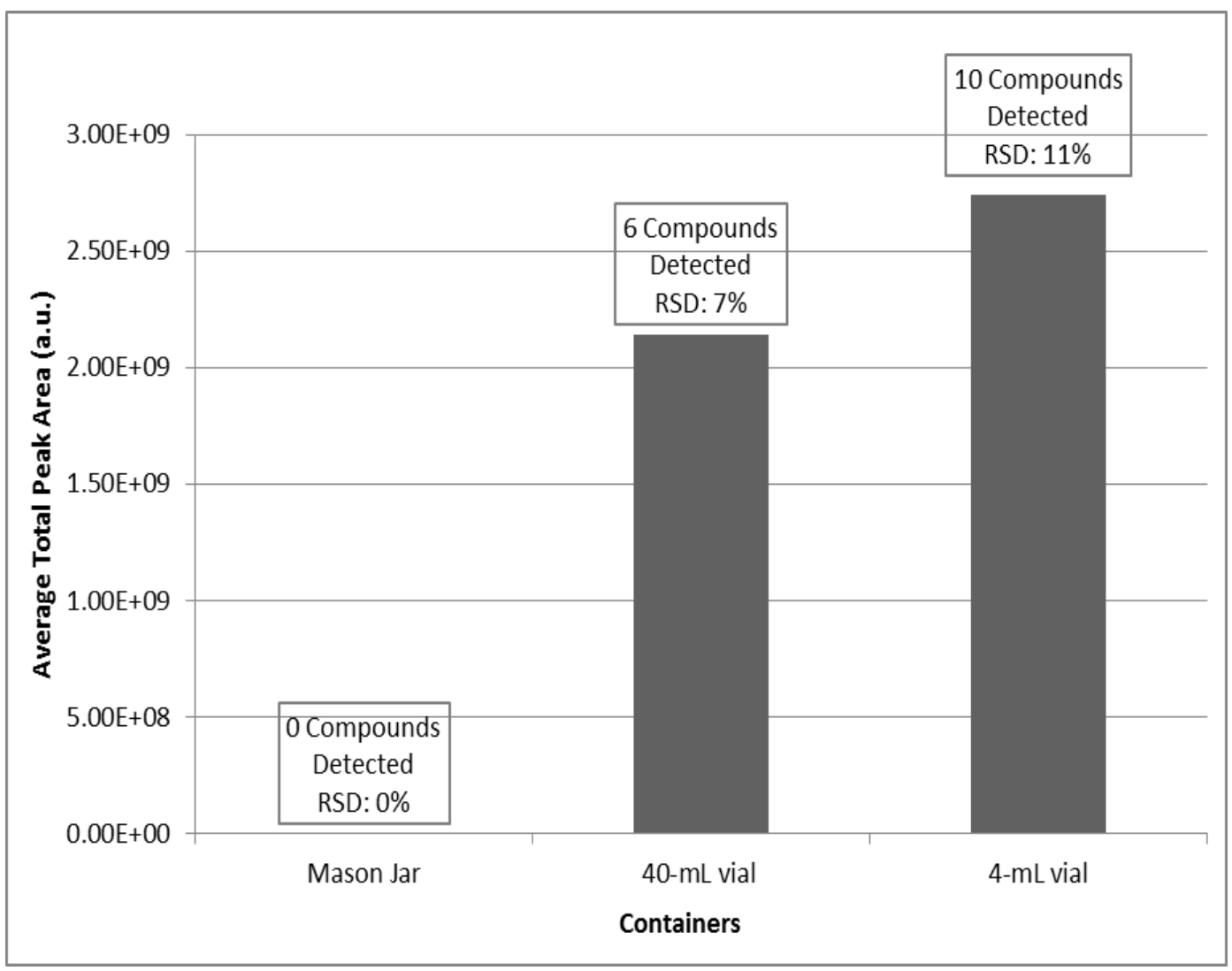

Figure 19. Comparison of the Compounds Extracted when the Container Volume is modified

Modification of the analytical method developed within the GC instrument was performed. Gas Chromatography is an analytical method that uses a mobile gas and undergoes separation through temperature controls. Method A was initially used for preliminary collection, however the resolution of the peaks were relatively low causing a challenge in the identification of various unknown analytes detected. The ramping of the method was adjusted to determine whether parameters would maximize the Signal to Noise Ratio, increase peak resolution, and decrease tailing and fronting. During modification a 50ppm solution was created consisting of five compounds: 1,3benzodioxole, 2,4-bis (1,1-dimethylethyl)-phenol , 4- 
bromomethylenedioxypropiohonene, methylone, and MDPV. The drug standards were chosen because structurally they included similarities and variations amongst each other; like various types of bath salts on the market. Detection of the parent drug was another key objective, so the analytical method was modified with its identification in mind. The other standards were used on the basis of their identification within the sample since preliminary stages and also resulted in large peaks, tailing, and coelution with neighboring chromatographic peaks. The varying methods tested included:

Method A: Split less mode; Initial temperature oven was held at $40^{\circ} \mathrm{C}$ for two minutes, ramped at $10^{\circ} \mathrm{C} / \mathrm{min}$ until the temperature of $280^{\circ} \mathrm{C}$ was reached and held for five minutes.

Method B: Split mode 20:1; Initial temperature oven was held at $40^{\circ} \mathrm{C}$ for two minutes, ramped at $10^{\circ} \mathrm{C} / \mathrm{min}$ until the temperature of $280^{\circ} \mathrm{C}$ was reached and held for five minutes.

Method C: Split mode 20:1; Initial temperature oven was held at $35^{\circ} \mathrm{C}$ for five minutes, ramped at $10^{\circ} \mathrm{C} / \mathrm{min}$ until the temperature of $260^{\circ} \mathrm{C}$ was reached and held for twelve minutes.

Method D: Split mode 20:1; Initial temperature oven was held at $40^{\circ} \mathrm{C}$ for five minutes, ramped at $10^{\circ} \mathrm{C} /$ min until the temperature of $250^{\circ} \mathrm{C}$ was reached and held for four minutes, ramped to $260^{\circ} \mathrm{C}$ at $10^{\circ} \mathrm{C} / \mathrm{min}$ and held for 2 minutes.

Analytical conclusions drawn from the modification of the temperature programming in the Bruker, proved futile as none of the methods improved the resolution 
of the compounds, beyond the abundance obtained in method C. As depicted in figure 20 , the separation of both drug standards were not obtained with all method modifications attempted. Peak coelution experienced was a result of the fast elution of the compounds off the nonpolar column. Synthetic cathinones are polar drugs bearing amines and carbonyl groups. Increasing the polarity of the column would retain the compounds thus allowing for greater separation of similar components. A Sol-gel wax, polar, column was installed and the volatiles extracted for an hour were run on the column using the same method.

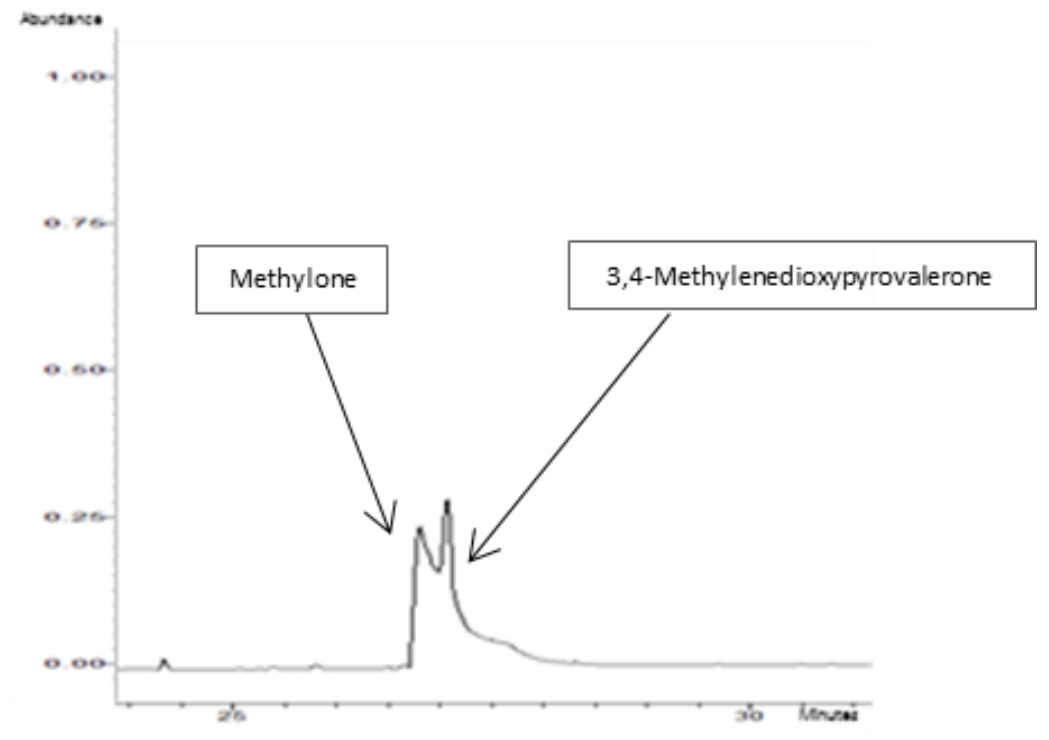

Figure 20. Peak Resolution of Methylone and MDPV on a Non-Polar Column

Conducting the analysis using polar column allowed for an increased resolution of approximately $92 \%$ in comparison to the non-polar column. Further manipulation of the temperature programing and split ratio, the optimal GC method used for the analysis of synthetic cathinones was determined to be the following: 
Method: Split mode off for 1 minute and then at a ratio of 10:1; Initial temperature oven was held at $40^{\circ} \mathrm{C}$ for five minutes, ramped at $15^{\circ} \mathrm{C} / \mathrm{min}$ until the temperature of $240^{\circ} \mathrm{C}$ was reached and held for six minutes; ramped to $250^{\circ} \mathrm{C}$ at $10^{\circ} \mathrm{C} / \mathrm{min}$ and held for 2 minutes; for a total run time of 27.33 minutes. Mass Scan Range: $35-350 \mathrm{~m} / \mathrm{z}$; electron ionization mode.

Establishing a smaller volumetric containment system and analytical method allowed for an enhanced extraction and separation of the volatiles prematurely identified in the cathinone derivative sample. Following further GC optimization for the method, the same preliminary extraction was performed on the methylone case. The volatiles identified initially were compared to the organic compounds found within the headspace of two drugs MDMA and methamphetamine (table 10 and 11). Notably these three classes of drugs are molecular similar in structure. The synthetic cathinone backbone differs by the addition of a carbonyl group. The VOCs identified within the extraction of the methylone case were compared to known VOCs identified from the headspace extraction of MDMA/ Methamphetamine, and a premature extraction of MDMA and Methamphetamine samples collected from the Florida Highway Patrol (FHP). The extractions of the FHP samples were placed in 4-mL vials and equilibrated for 24 hours and extracted for 1 hour. 
Table 10. VOC Comparison of Methylone, FHP MDMA Sample, and Literature Findings of Volatiles from MDMA Samples

\begin{tabular}{|c|c|c|c|}
\hline Compounds & Macias Report $^{82}$ & $\begin{array}{c}\text { FHP Sample of } \\
\text { MDMA }\end{array}$ & $\begin{array}{c}\text { Bath Salt } \\
\text { Sample }\end{array}$ \\
\hline Methamphetamine $\mathrm{HCl}$ & $\mathrm{X}$ & $\mathrm{X}$ & \\
\hline Isosafrole & $\mathrm{X}$ & $\mathrm{X}$ & \\
\hline Piperonal & $\mathrm{X}$ & $\mathrm{X}$ & $\mathrm{X}$ \\
\hline Piperonyl Alcohol & $\mathrm{X}$ & & \\
\hline MDP-2-POH & $\mathrm{X}$ & $\mathrm{X}$ & \\
\hline MDP-2-P & $\mathrm{X}$ & $\mathrm{X}$ & \\
\hline MDMA & $\mathrm{X}$ & $\mathrm{X}$ & \\
\hline MDEA & $\mathrm{X}$ & & \\
\hline Butylated Hydroxytoluene & $\mathrm{X}$ & $\mathrm{X}$ & $\mathrm{X}$ \\
\hline Acetophenone & & $\mathrm{X}$ & \\
\hline 2-Ethyl-1-hexanol & & $\mathrm{X}$ & $\mathrm{X}$ \\
\hline $\begin{array}{l}\text { 2,6-Dimethoxy } \\
\text { Acetophenone }\end{array}$ & & $\mathrm{X}$ & \\
\hline
\end{tabular}


Table 11. VOC Comparison of Methylone, FHP Methamphetamine Sample, and Literature Findings of Volatiles from Methamphetamine Samples

\begin{tabular}{|c|c|c|c|}
\hline Compounds & Vu Report & $\begin{array}{c}\text { FHP Sample of } \\
\text { Methamphetamine }\end{array}$ & $\begin{array}{c}\text { Bath Salt } \\
\text { Sample }\end{array}$ \\
\hline Methamphetamine HCl & $\mathrm{X}$ & $\mathrm{X}$ & \\
\hline 2-methylbenzofuran & $\mathrm{X}$ & & \\
\hline Benzaldehyde & $\mathrm{X}$ & $\mathrm{X}$ & \\
\hline Methyl benzoate & $\mathrm{X}$ & $\mathrm{X}$ & \\
\hline Benzyl chloride & $\mathrm{X}$ & $\mathrm{X}$ & \\
\hline $\begin{array}{c}\text { 1-Chloro-1-phenyl-2- } \\
\text { propanone }\end{array}$ & $\mathrm{X}$ & & \\
\hline benzene & $\mathrm{X}$ & $\mathrm{X}$ & \\
\hline 1-phenyl-2-propanone & $\mathrm{X}$ & $\mathrm{X}$ & \\
\hline $\begin{array}{c}\text { Butylated Hydroxytoluene } \\
\text { 4-hydroxy-4-methyl-2- } \\
\text { pentanone }\end{array}$ & $\mathrm{X}$ & $\mathrm{X}$ & \\
\hline Toluene & $\mathrm{X}$ & & \\
\hline
\end{tabular}

Comparing the methylone sample to the MDMA resulted in much more similarity in comparison to the methamphetamine sample. During analysis there was only one compound that was encountered when examining the headspace and compared to the known literature values. For the methamphetamine samples, approximately $55 \%$ of the volatiles found in the samples analyzed under the $\mathrm{Vu}$ report were also found in the sample analyzed from the FHP. Comparison of the MDMA samples to the bath salt case also revealed a similar trend. Approximately $50 \%$ of the volatiles identified were found in both the FHP sample and the volatiles found within the literature report. Only $11 \%$ and $19 \%$ of the volatiles found in the methylone case were also found in the headspace of the 
samples collected within the research study and the sample analyzed from the patrol unit (respectively). Preliminary results highlighted that although the parent drugs do bare some structural resemblance, the volatiles identified within the headspace of each case suggest few reoccurrences.

\subsubsection{Development of a Chemical Ionization Mass Spectrometry Method}

During the preliminary extraction of volatiles found in the headspace of these synthetic drugs, the library limitation was a challenging factor. Although the retention times of various peaks were different, the identification of several peaks was classified as the same compound based on the fragmentation and suggestive results. Conducting analysis under electron ionization mode $(70 \mathrm{eV})$ resulted in serve fragmentation among the compounds yielding similar patterns. As a result, the suggestive searches presented the same conclusion, for different compounds; indicated by the retention times which depend on the elution rate from the column. An elution which solely depends on a compound's binding affinity to the stationary phase. Analysis under electron ionization allowed the sample to come into direct contact with fast accelerating electrons, permitting severe ionization of any sample within the chamber through a transfer of energy. Unlike EI, complimentary chemical ionization is a soft ionization technique that allows for the molecular ion to remain intact; figure 21. Bombarding with ionized chemical reagents instead, the compound isn't broken down as severely and the whole molecular structure is more likely to continue into the detector to be multiplied and analyzed. This molecular information can assist with narrowing down the list of possible compounds presented from the chromatographic run analyzed in electron ionization mode. 


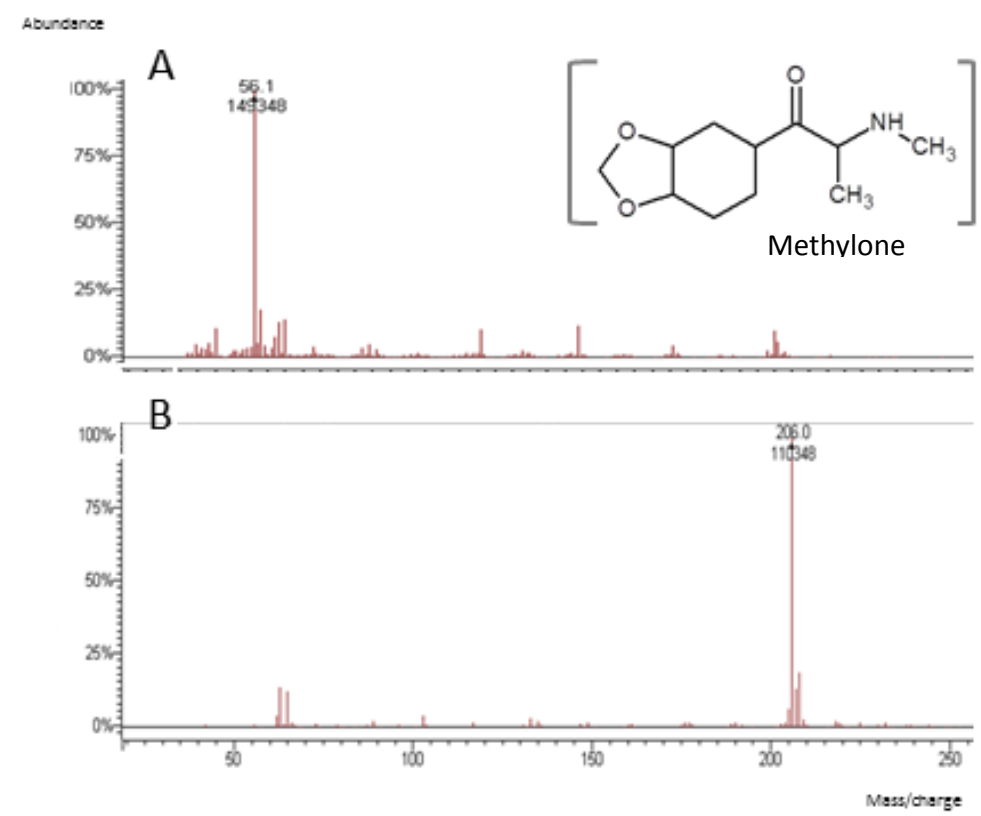

Figure 21. A. The Mass Spectrum of Methylone under Electron Ionization Mode. B. The Mass Spectrum of Methylone under Chemical Ionization Mode

This study focused on using CI as a complimentary technique to the primary analysis conducted on the synthetic cathinones. In order to achieve soft ionization a Varian CP 3800 Saturn 2000 MS-Ion trap was employed. Proper functionality of CI is based on six parameters: Storage Level, Ejection Amplitude, Background Mass (BM), Target Ion Count (TIC), Maximum Ionization Time (Max IT), and Maximum Reaction Time (Max RT). Of these, five were the primary focus. The target TIC which determines the number of ions allowed in the ion trap was kept at a preset limit, unmodified, as suggested by the user manual. Background Mass selected within the software is typically equal to or greater than the mass of the largest reagent ion produced by the chosen reagent chemical. All masses lower than this value will be ejected from the ion trap. Each default setting for the BM was predetermined to use the value that would only include the 
base minimum of each protonated reagent for successful ionization. This value was slightly optimized to determine whether decreasing the value would heighten the presence of undesired reagent ions or if an increase would be subjected to any loss of the simple protonated reagent ion.

The CI storage level determines the smallest mass stored in the trap during the ionization of the reagent gas and the lowest mass stored during reaction. This level is usually lower than the mass of the reagent gas of interest. However, it can range from 5$150 \mathrm{~m} / \mathrm{z}$. Selection of an adequate storage level is typically dictated by the reagent chosen. The ejection amplitude is the voltage that establishes the lowest mass cut off. The voltage is usually slightly higher than the largest reagent ion and ejects all unwanted ions prior to the $\mathrm{CI}$ reaction. The storage level and ejection amplitude are two parameters that work together. With a direct correlation, when one parameter increases the other must do so as well. Failure to insufficiently monitor these two parameters together will cause CI to fail.

The last two parameters optimized were Max IT and Max RT. As indicated by the name, Max IT is the maximum amount of time given for the energetic electrons emitted from the filament to interact with the chosen reagent to produced reagent ions. Max RT is the maximum given amount of time for the reagent ions to react with the sample to produce the sample ions. The range of time span given for each parameter is between 10 to $2500 \mu$ s and 1 to $128 \mathrm{msec}$; respectively.

Prior to analysis, the same standard testing pool employed during the chromatographic developmental (section 5.1) stage was used. A 50ppm solution was 
created for the standards and stored in the fridge between runs. The first parameters optimized were the storage level and the ejection amplitude. These parameters were analyzed first to follow the progression of the CI process. In order for soft ionization to occur the first step is the removal of undesired reagent molecules for the ionization reaction. This removal requires set values of both storage level and ejection amplitude. After these parameters, optimization continued along with the background mass, ionization time, and reaction time. The liquid reagents systematically compatible with the Varian software and therefore evaluated were Ammonia, Methanol, and Acetonitrile.

Ammonia was the first reagent evaluated. To produce the gas required approximately $2 \mathrm{~mL}$ of Ammonium Hydroxide was placed in the CI bulb in the back of the MS receiver. The system was given 15 minutes for the gas to generate and migrate into the values of the mass spectrometer. A daily checks method was loaded on the software to allow manual control of the system to optimize the influx of reagent gas into the trap. The CI gas needle, located at the front of the mass spectrometer, was adjusted until the pressure produced a 10:1 ratio of the peak heights at mass $18\left[\left(\mathrm{NH}_{3}\right) \mathrm{H}^{+}\right]$to 17 $\left(\mathrm{NH}_{3}{ }^{+}\right)$approximately. The default settings of both the storage level and ejection amplitude for the reagent were $13 \mathrm{~m} / \mathrm{z}$ and $9 \mathrm{v}$. The deviations of the method are listed in table 12.

Initially, the storage level was kept constant to determine the sole effect of voltage fluctuation on the reaction's sensitivity. Afterwards, the storage level was modified slightly higher and lower than the mass of ammonia, $17 \mathrm{~g} / \mathrm{mol}$. In response to these alterations, the ejection amplitude was initially maintained to determine if the separation 
of values would have a positive or negative effect on the ionization and also slightly increased along with the storage level. The detection of each standard was compared amongst the deviating spectral methods.

Table 12. Chemical Ionization (Ammonia) Parameter Modification of Storage Level and Ejection Amplitude

\begin{tabular}{|c|c|c|c|c|c|c|}
\hline Method Name & $\begin{array}{c}\text { Storage } \\
\text { Level }\end{array}$ & $\begin{array}{c}\text { Ejection } \\
\text { Amp }\end{array}$ & BM & TIC & $\begin{array}{c}\text { Max } \\
\text { IT }\end{array}$ & $\begin{array}{c}\text { Max } \\
\text { RT }\end{array}$ \\
\hline Ammonia Method1 & 13 & 9 & 45 & 5000 & 2000 & 60 \\
\hline Ammonia Method2 & 13 & 12 & 45 & 5000 & 2000 & 60 \\
\hline Ammonia Method3 & 13 & 7 & 45 & 5000 & 2000 & 60 \\
\hline Ammonia Method4 & 16 & 9 & 45 & 5000 & 2000 & 60 \\
\hline Ammonia Method5 & 16 & 12 & 45 & 5000 & 2000 & 60 \\
\hline Ammonia Method6 & 16 & 7 & 45 & 5000 & 2000 & 60 \\
\hline Ammonia Method7 & 19 & 9 & 45 & 5000 & 2000 & 60 \\
\hline Ammonia Method8 & 19 & 7 & 45 & 5000 & 2000 & 60 \\
\hline Ammonia Method9 & 19 & 12 & 45 & 5000 & 2000 & 60 \\
\hline
\end{tabular}

The default settings tested in method one were pre-established by the software when the reagent ammonia was selected. Initial observations within this method indicated detection and integration limitations of 4-Bromo-1,2(methylenedioxy) benzene. Neither this compound nor methylone were detected chromatographically and spectrally within this method (figure 23). In general both the default and method 7 suffered from sensitivity issues. An explanation for the decrease in sensitivity noted for method 7, can be summed up to the large difference between the storage level and ejection amplitude $(19 \mathrm{~m} / \mathrm{z}$ and $9 \mathrm{v})$, causing insufficient ionization for the creation and detection of corresponding positive ions. The same phenomenon was experienced with both method 8 and 9; where each method possessed a storage level setting of $19 \mathrm{~m} / \mathrm{z}$ with varying ejection voltages. Through experimentation it was determined that a storage level of 
$19 \mathrm{~m} / \mathrm{z}$ would cause a decrease in sensitivity for all five standards analyzed, thus eliminating method 7-9 from consideration.

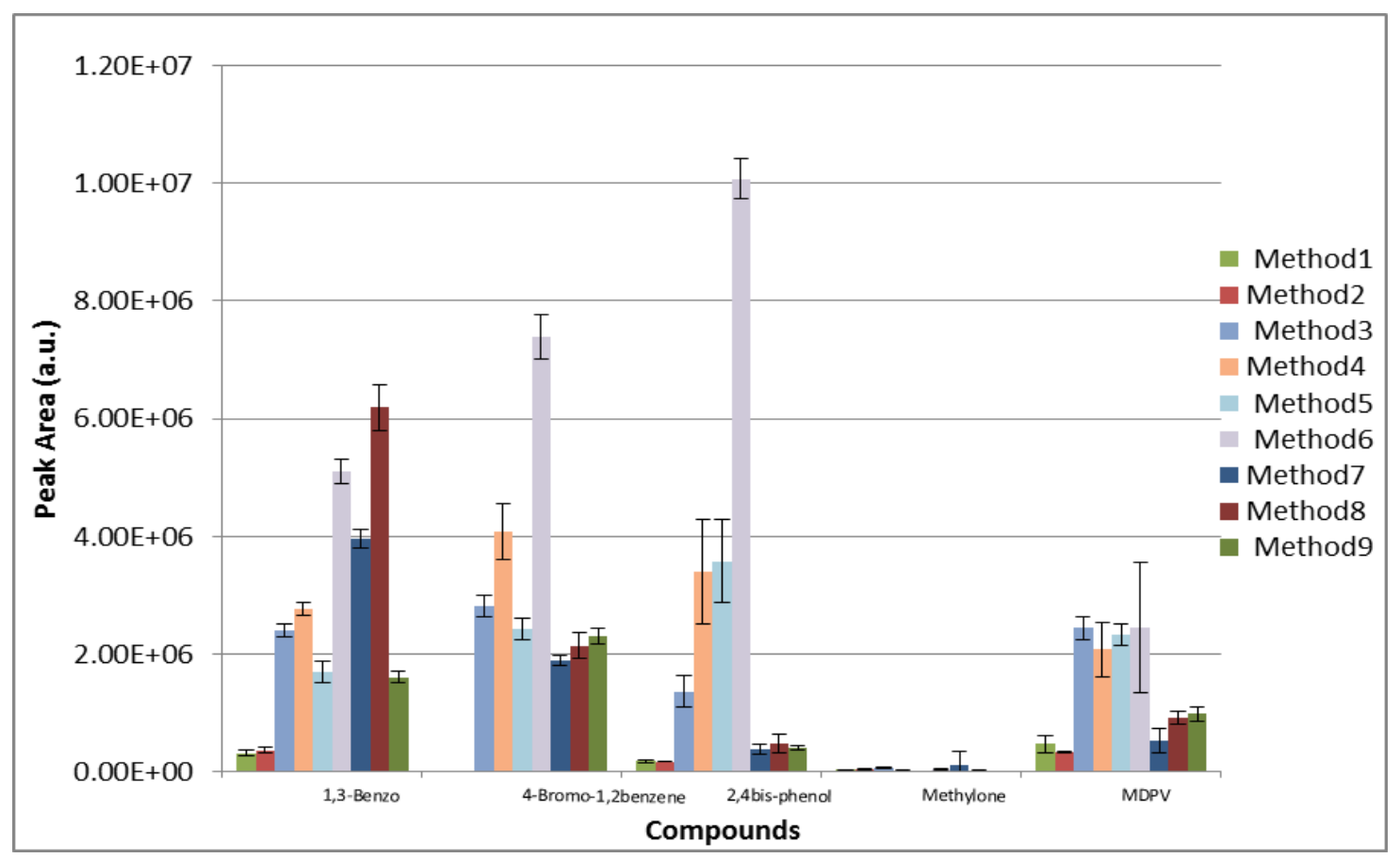

Figure 22. CI-Ammonia: Storage Level and Ejection Amplitude Parameter Optimization

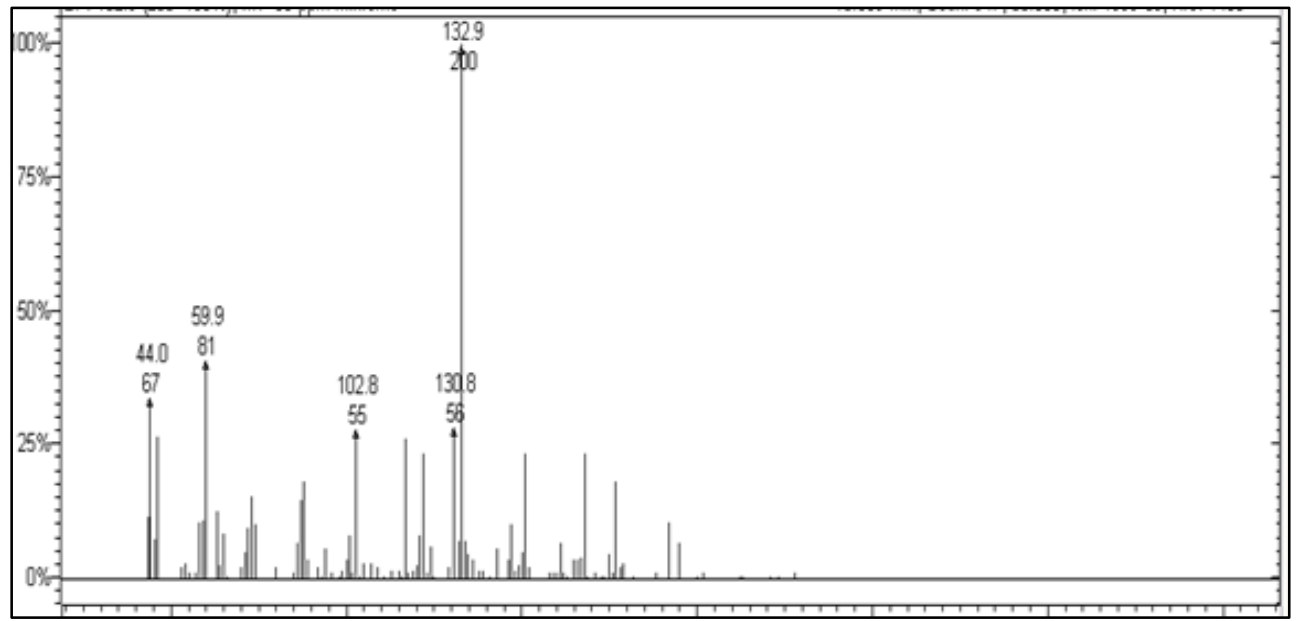

Figure 23. Indistinguishable Mass Spectrum for 4-Bromo-1,2(methylenedioxy) benzene Operated under the Default Method of Ammonia 
Quantitative and qualitative comparisons of methods 2- 6 showed the largest abundance detected within the sixth method $(16 \mathrm{~m} / \mathrm{z} ; 7 \mathrm{ev})$; followed by methods 3,4 , and 5. While all previously identified methods were characterized by an increase in the storage level, all voltages were either simultaneously increased or decreased from the trend to test the ionization's stability. With no method incurring a greater degree of separation than method 7, all compounds remained detectable. Although, significantly different in ionization and detection capabilities, none of the methods were able to provide any spectral information (limiting factor similar to method 8 and 9). The large abundances identified were produced by the extensive interaction between the reagent ion and the sample, causing the molecular ion to fragment down into smaller components. Although chromatographically there was a decrease in abundance for method 2, this method was the richest spectrally. Although the ammonia reagent failed to yield a generous amount of spectral information, the settings in method 2 proved to be a good direction to address this weakness. Therefore quantitatively the abundance was sacrificed for the qualitatively information seen in this method.

Given the new values for the storage level and ejection amplitude, the background mass was modified to accommodate a ranges of masses listed in table 13. From the quantitative results yielded in figure 24 , there was statistical findings to suggest a large difference in the analytical detection accomplished within method 14 in comparison to the other methods (ANOVA F calculated: 3.061173 and $f$ critical:2.028518). Methods 1012 were removed from consideration as there were problems detecting 4-Bromo-1,2(methylenedioxy) benzene. The remaining methods were more reproducible and showed no limitation in accurately identifying and detecting the mixture's components. By 
comparing methods 13 and 14 (both quantitatively stronger than method 2) there was no increase in the molecular information obtained when the BM was increased from 60 to 65 $\mathrm{m} / \mathrm{z}$. This lack of qualitative difference was also supported quantitatively through $\mathrm{t}$-test ( $\mathrm{t}$ critical 0.4132 ) which showed there was no statistical difference between each method's detection, at a confidence level of $95 \%$. While more sensitive than method 2 and demonstrating a similar spectrum, method 13 proved to be a solid candidate to proceed with optimization as it was both quantitatively and qualitatively superior.

Table 13. Chemical Ionization (Ammonia) Parameter Modification of Background Mass (BM)

\begin{tabular}{|c|c|c|c|c|c|c|}
\hline Method Name & $\begin{array}{c}\text { Storage } \\
\text { Level }\end{array}$ & $\begin{array}{c}\text { Ejection } \\
\text { Amp }\end{array}$ & BM & TIC & $\begin{array}{c}\text { Max } \\
\text { IT }\end{array}$ & $\begin{array}{c}\text { Max } \\
\text { RT }\end{array}$ \\
\hline Ammonia Method2 & 13 & 12 & 45 & 5000 & 2000 & 60 \\
\hline Ammonia Method10 & 13 & 12 & 50 & 5000 & 2000 & 60 \\
\hline Ammonia Method11 & 13 & 12 & 55 & 5000 & 2000 & 60 \\
\hline Ammonia Method12 & 13 & 12 & 40 & 5000 & 2000 & 60 \\
\hline Ammonia Method13 & 13 & 12 & 60 & 5000 & 2000 & 60 \\
\hline Ammonia Method14 & 13 & 12 & 65 & 5000 & 2000 & 60 \\
\hline
\end{tabular}




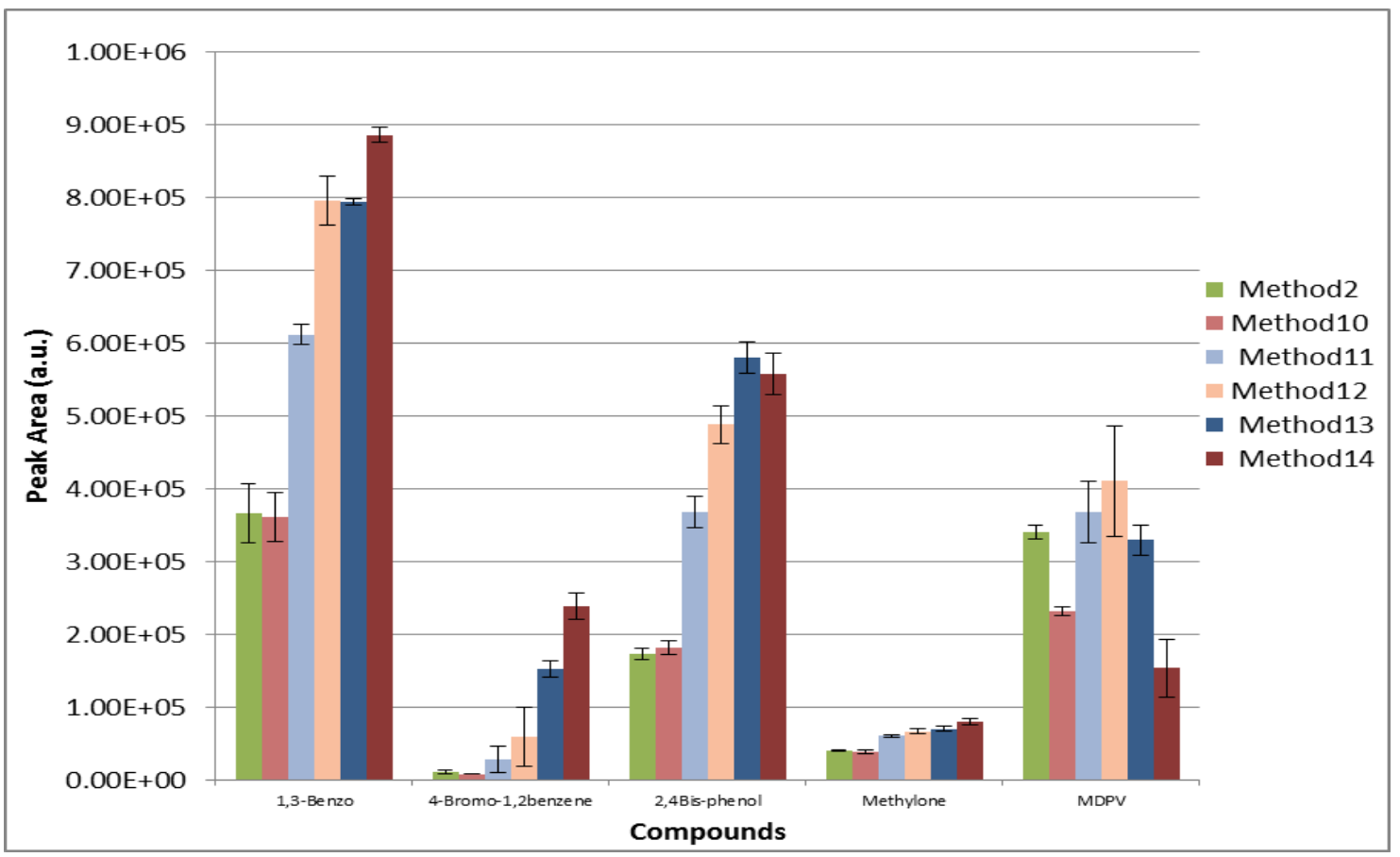

Figure 24. CI-Ammonia: Background Mass Parameter Optimization

Variation of the ionization time, as displayed in table 14, was kept between the ranges of $1000-2500 \mu \mathrm{s}$. With a maximum operating time of $2500 \mu \mathrm{s}$, the software's manual indicated possible damage incurring at values beyond this point. During examination it was also noted that times lower than $1000 \mu$ s also suffered from unsuccessful completion of ionization and detection. Initial observations of the methods exhibited a proportional relationship between the ionization time and instrument's sensitivity. An increase or decrease in the maximum ionization time was also characterized by an increase and decrease (respectively) in each analyte's detection for the corresponding method. Methods 16 and 17 were the first to be eliminated for the significant decrease in the abundance determined. This quantitative decrease was also 
coupled by a decrease in the molecular information that was obtained through each method.

During parameter modification it was noted that by increasing the ionization time to $2500 \mu \mathrm{s}$, the largest abundance for each analyte was detected in method 15. Spectrum analysis between this method and number 13 (second most dominating method chromatographically) were compared. Through statistical testing (95\% confidence) it was determined that there was no significant difference from increasing the ionization time from 2000 to $2500 \mu \mathrm{s}$ ( $\mathrm{t}=0.02067)$. However by increasing the time there was a downward shift in the molecular information obtained. During the evaluation it was noted that the fragment ratio (figure 25) indicating the molecular weight of the compound, although still present, was no longer the base peak in the spectrums analyzed for method 15. Overall, there was no scientific benefit in the modification of the ionization time beyond the default settings indicated within the software. As a result, the findings supported the notion that method 13 remained the optimal method to proceed with. 


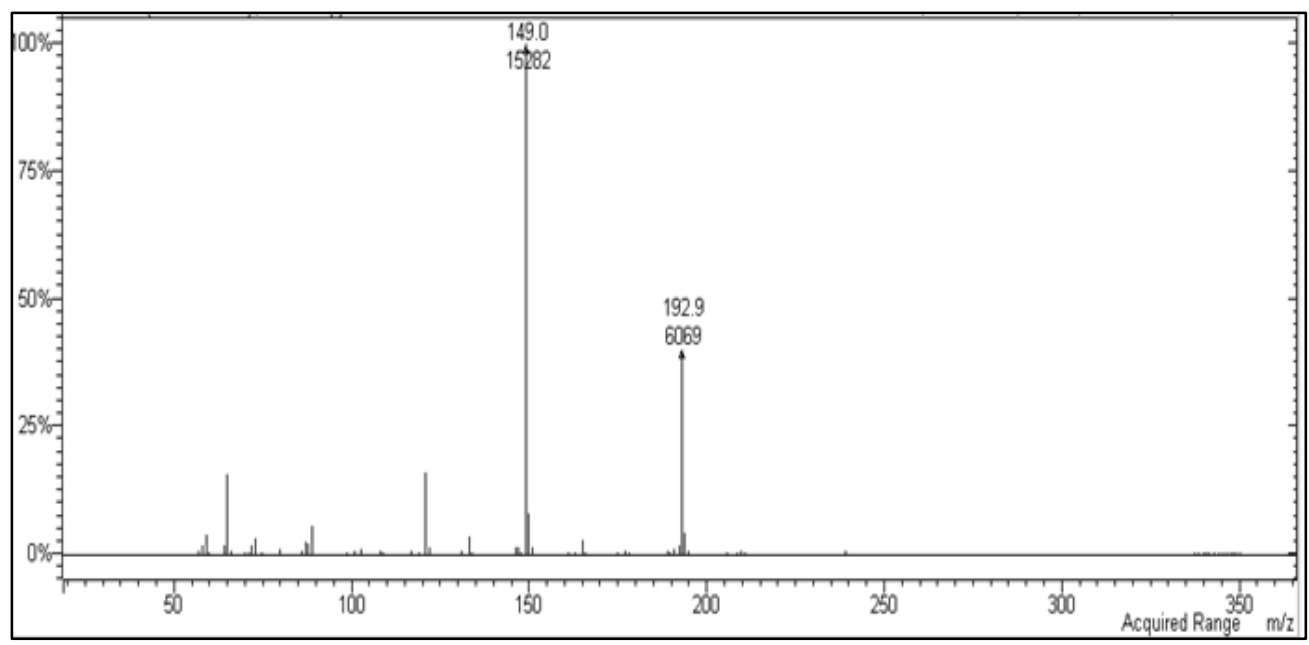

Figure 25. Molecular Spectrum Obtained for Methylone under the Parameters of Method 15

Table 14. Chemical Ionization (Ammonia) Parameter Modification of Maximum Ionization Time (Max IT)

\begin{tabular}{|c|c|c|c|c|c|c|}
\hline Method Name & $\begin{array}{c}\text { Storage } \\
\text { Level }\end{array}$ & $\begin{array}{c}\text { Ejection } \\
\text { Amp }\end{array}$ & BM & TIC & $\begin{array}{c}\text { Max } \\
\text { IT }\end{array}$ & $\begin{array}{c}\text { Max } \\
\text { RT }\end{array}$ \\
\hline Ammonia Method13 & 13 & 12 & 60 & 5000 & 2000 & 60 \\
\hline Ammonia Method15 & 13 & 12 & 60 & 5000 & 2500 & 60 \\
\hline Ammonia Method16 & 13 & 12 & 60 & 5000 & 1500 & 60 \\
\hline Ammonia Method17 & 13 & 12 & 60 & 5000 & 1000 & 60 \\
\hline
\end{tabular}




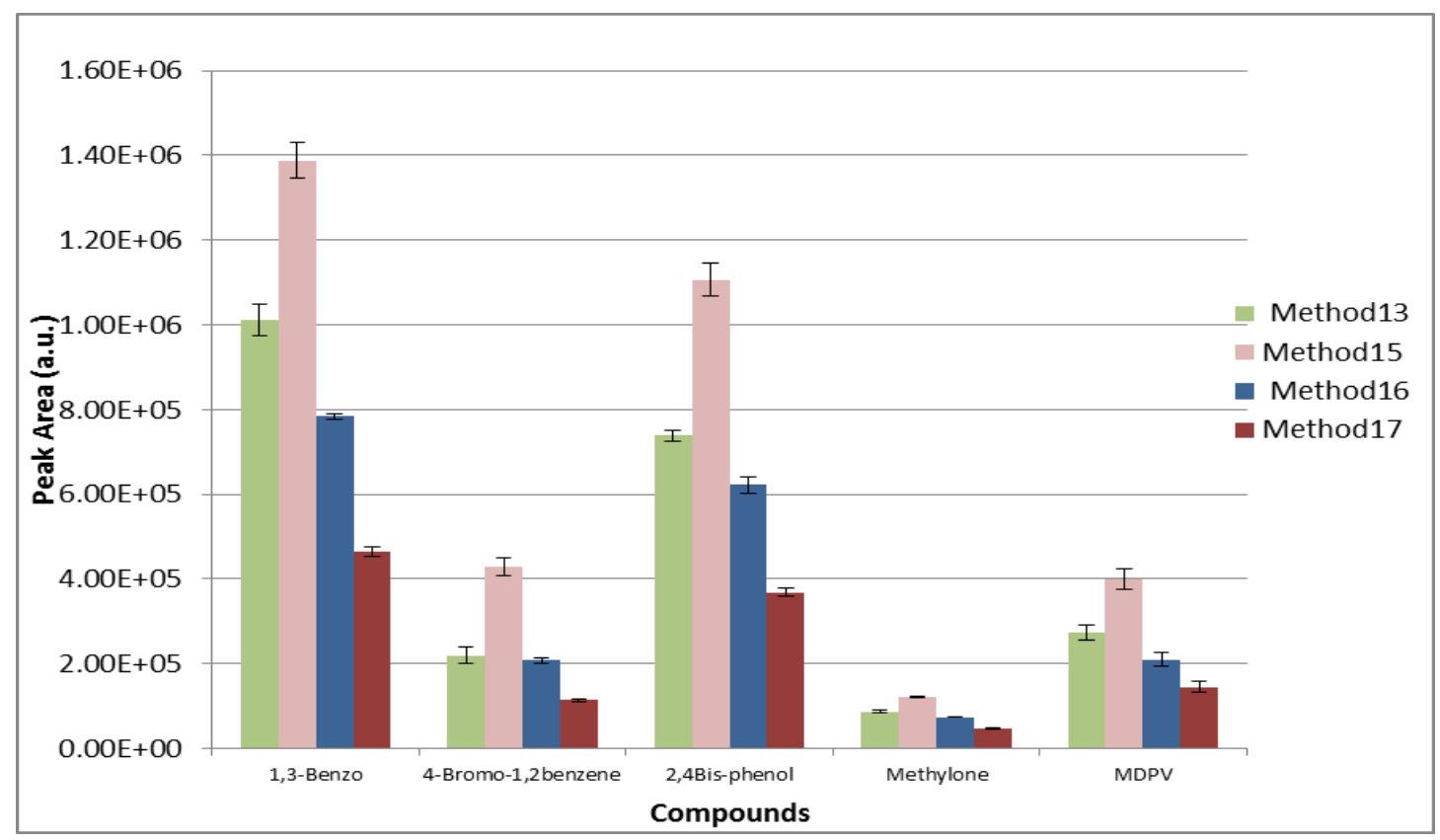

Figure 26. CI-Ammonia: Maximum Ionization Time Parameter Optimization

The last parameter examined for the CI reagent Ammonia was the Maximum Reaction time (Max RT). In theory the maximum time was increased and decreased by a total factor of 10 (5 increments). When setting the reaction time (table 15) it was hypothesized that increasing the reaction length would result in an increase in abundance. This increase would be caused by a prolonged interaction between the reagent ion and standard, but would also result in an increase in the fragments. As a result the fragmentation pattern would be similar to the electron ionization mode. As seen in figure 27, the hypothesized statement was rejected.

Method 19, solely increased by a factor of five, experienced a significant decrease in analyte detection. However with this decline in sensitivity, $60 \%$ of the compounds remained detectable within this method. For all methods with a reaction time higher than 
65 and lower than $60 \mathrm{msec}$ there was no chromatographic or spectral findings to support the ionization and detection of the five standards. Findings from this experimentation indicated that the default value predetermined from the software for the reaction time, was the most valuable for the completion of the ionization process and detection thereafter. As a result, with the Ammonia reagent, it was determined that the most suitable parameters would be those highlighted from the Ammonia Method13 (table 15).

Table 15. Chemical Ionization (Ammonia) Parameter Modification of Maximum Reaction Time (Max RT)

\begin{tabular}{|c|c|c|c|c|c|c|}
\hline Method Name & $\begin{array}{c}\text { Storage } \\
\text { Level }\end{array}$ & $\begin{array}{c}\text { Ejection } \\
\text { Amp }\end{array}$ & BM & TIC & $\begin{array}{c}\text { Max } \\
\text { IT }\end{array}$ & $\begin{array}{c}\text { Max } \\
\text { RT }\end{array}$ \\
\hline Ammonia Method13 & 13 & 12 & 60 & 5000 & 2000 & 60 \\
\hline Ammonia Method19 & 13 & 12 & 60 & 5000 & 2000 & 65 \\
\hline Ammonia Method20 & 13 & 12 & 60 & 5000 & 2000 & 70 \\
\hline Ammonia Method21 & 13 & 12 & 60 & 5000 & 2000 & 55 \\
\hline Ammonia Method22 & 13 & 12 & 60 & 5000 & 2000 & 50 \\
\hline Ammonia Method23 & 13 & 12 & 60 & 5000 & 2000 & 45 \\
\hline
\end{tabular}




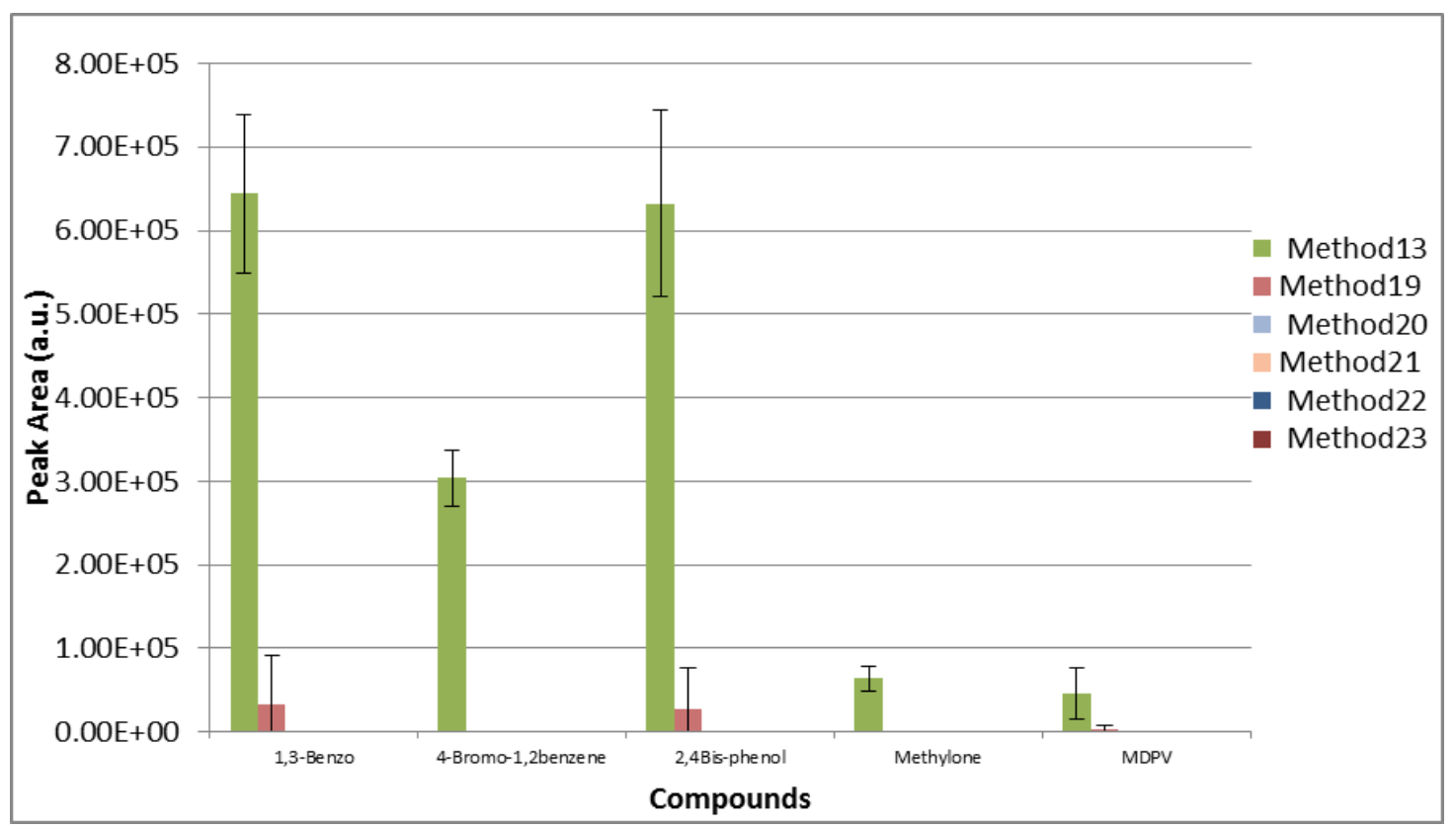

Figure 27.CI-Ammonia: Maximum Reaction Time Parameter Optimization

After optimization was completed for Ammonia, the next reagent (Methanol) was loaded into the system's bulb. The valve's settings were adjusted until a mass of $33 \mathrm{~m} / \mathrm{z}$ was identified as the base peak. Like Ammonia the ordering of optimization began with the storage level and ejection amplitude. The default method established optimal storage and ejection at $19 \mathrm{~m} / \mathrm{z}$ and $15 \mathrm{v}$ respectively (table 16). Following a similar protocol to the Ammonia trials the storage level initially remained constant will varying the ejection amplitude. Afterwards the storage level was slightly increased along with fluctuations in the voltage. Unlike the storage levels set in the ammonia trials, during the methanol testing this level remained under the mass of the reagent as suggested by the manual. Prior investigations, as shown above, hindered the ionization process when the level was above the mass. 
Table 16. Chemical Ionization (Methanol) Parameter Modification of Storage Level and Ejection Amplitude.

\begin{tabular}{|l|c|c|c|c|c|c|}
\hline Method Name & $\begin{array}{c}\text { Storage } \\
\text { Level }\end{array}$ & $\begin{array}{c}\text { Ejection } \\
\text { Amp }\end{array}$ & BM & TIC & $\begin{array}{c}\text { Max } \\
\text { IT }\end{array}$ & $\begin{array}{c}\text { Max } \\
\text { RT }\end{array}$ \\
\hline Methanol Method1 & 19 & 15 & 55 & 5000 & 2000 & 40 \\
\hline Methanol Method2 & 19 & 18 & 55 & 5000 & 2000 & 40 \\
\hline Methanol Method3 & 19 & 12 & 55 & 5000 & 2000 & 40 \\
\hline Methanol Method4 & 22 & 15 & 55 & 5000 & 2000 & 40 \\
\hline Methanol Method5 & 22 & 18 & 55 & 5000 & 2000 & 40 \\
\hline Methanol Method6 & 22 & 12 & 55 & 5000 & 2000 & 40 \\
\hline Methanol Method7 & 25 & 15 & 55 & 5000 & 2000 & 40 \\
\hline Methanol Method8 & 25 & 18 & 55 & 5000 & 2000 & 40 \\
\hline Methanol Method9 & 25 & 12 & 55 & 5000 & 2000 & 40 \\
\hline
\end{tabular}

The methanol reagent overall provided more dense spectral information in comparison to ammonia. Initial examination began with methods 6 and 9 as each resulted in the greatest sensitivity to each analyte introduced. The first eluting component, 1,3Benzodioxole, was characterized by a molecular peak at $122.7 / 121.9 \mathrm{~m} / \mathrm{z}$ and ion peak of $62.9 \mathrm{~m} / \mathrm{z}$. Although the molecular ion was present, this peak was insignificant and comparable to some of the smaller fragmentation peaks noted (figure 28). Method 6 also identified the molecular ion of 4-Bromo-1,2-(methylenedioxy)benzene as $202.8 \mathrm{~m} / \mathrm{z}$ with a base peak of $62.9 \mathrm{~m} / \mathrm{z}$. Beside these two fragments there was no other characterizing components to identify this compound. For this standard there was also a significant amount of spectrum noise that was noted after 202m/z. For 2,4-bis(1,1-dimethylethyl)phenol no molecular ion was identified. The only fragment showed within this spectrum belonged to $56.8 \mathrm{~m} / \mathrm{z}$. Such severity in fragmentation resulted in a similar pattern for EI. The molecular species of Methylone was identifiable as the base peak of $206 \mathrm{~m} / \mathrm{z}$. This ion was notably $1 \mathrm{~m} / \mathrm{z}$ lower than the reported literature molecular weight, however, this 
phenomenon was observed with other cathinone standards ran in CI mode; MDPV standard molecular ion was $275 \mathrm{~m} / \mathrm{z}$. Although there was slight noise present, the spectrum identified the molecular ion as the base peak at $275 \mathrm{~m} / \mathrm{z}$.

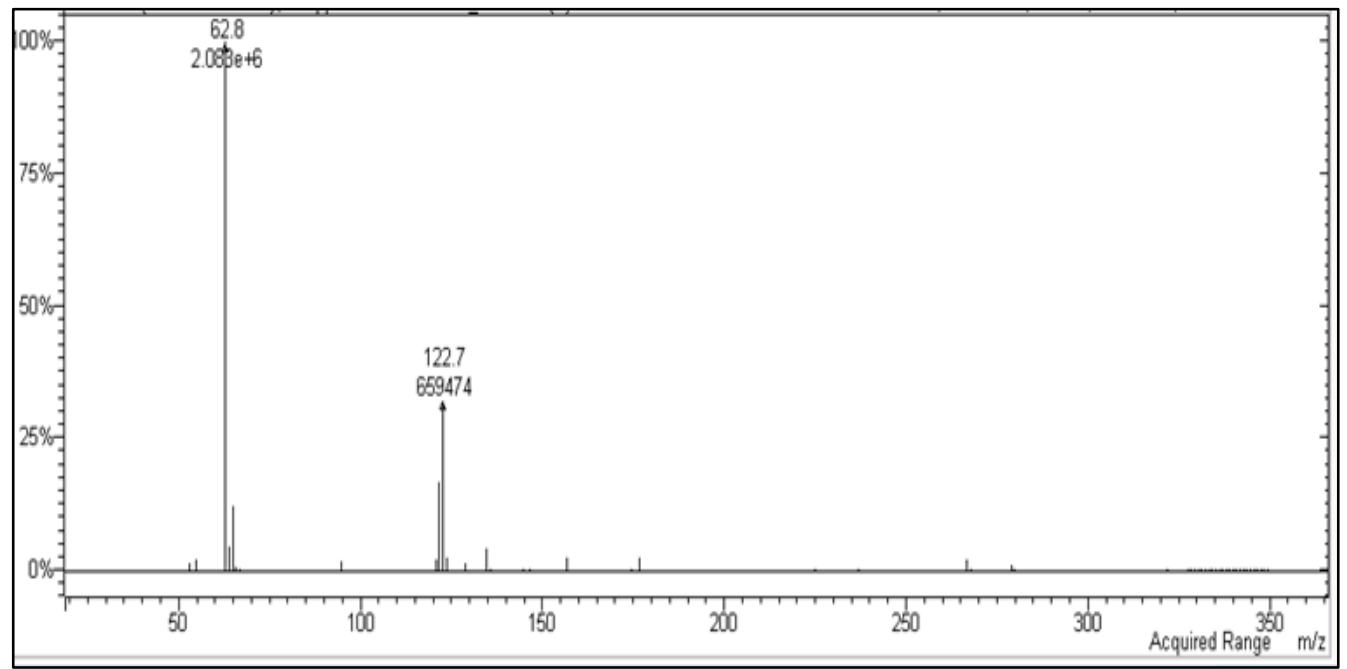

Figure 28. Mass Spectrum of 1,3-Benzodioxole Detected within Method 6

Unlike method 6, the spectra analyzed from method 9 were able to distinguish the molecular ion of 1,3-Benzodioxole as the base peak; in addition to a relatively good abundance for the fragment $62.9 \mathrm{~m} / \mathrm{z}$. Although smaller fragments were present, a major concern with this method was the fragmentation noise experienced after the mass ratio of $150 \mathrm{~m} / \mathrm{z}$; observed within triplicates (figure 29). This systematic noise created from operating under the parameters of method 9 may interfere with identifying unknown peaks by classifying misleading fragments as the molecular ion. Continuing analysis of 4Bromo-1,2-(methylenedioxy)benzene, identified the base peak as $62.9 \mathrm{~m} / \mathrm{z}$. The ion corresponding to the molecular weight had the same abundance as a non-factoring fragment of $64.8 \mathrm{~m} / \mathrm{z}$. For the remaining standards examined during this method the 
molecular mass was also lost. Each spectrum was clouded by a significant amount of background noise.

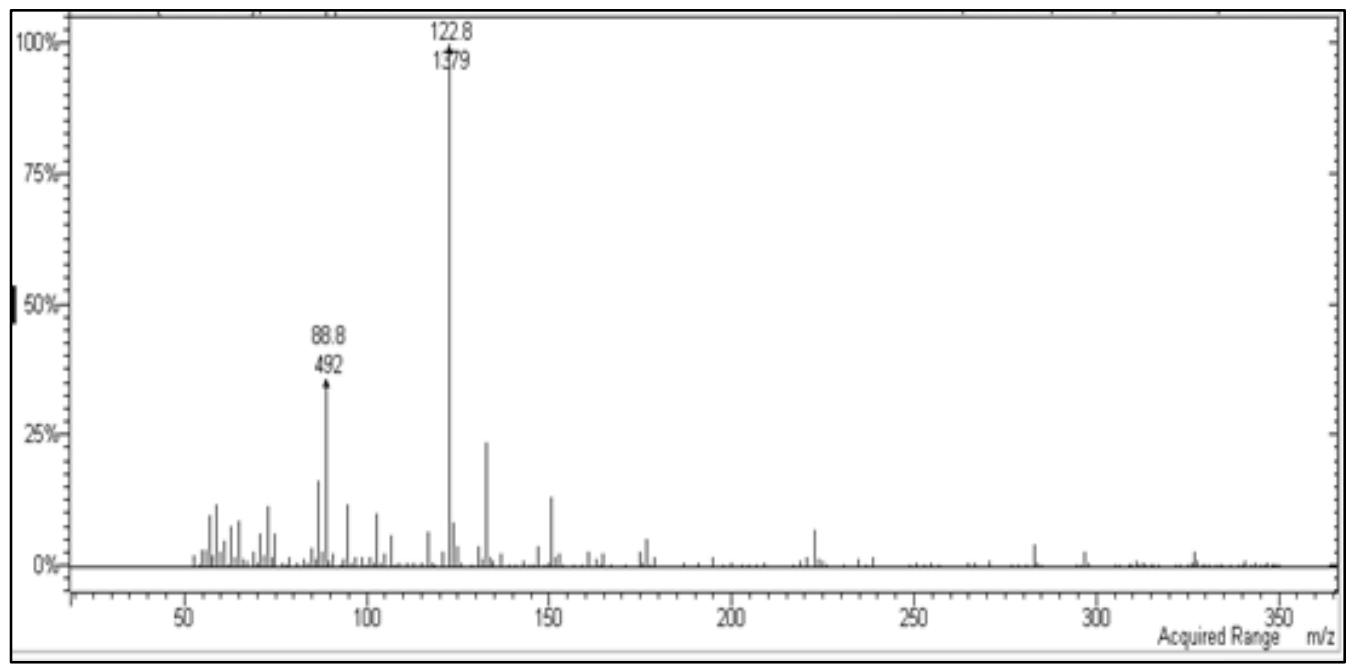

Figure 29. Mass Spectrum of 1,3-Benzodioxole Detected within Method 9

Aside from the spectral problems encountered in both method 6 and 9 , the remaining methods (with the exception of method 1 and 2) all experienced the present and integration of undesired peaks around 14.005 and 18.036 minutes. The peaks were unidentifiable and resulted in a spectrum with significant interference masking any obtainable information. Further examination suggested that the possible errors encountered as a result of these methods could hinder the identification of potential VOCs found in the drugs and were therefore eliminated.

After the exclusion of methods 3-9, the remaining two were evaluated together. Although the yield was quantitatively low these methods gave the best mass spectral information that could be obtained for each standard. Each molecular mass was identified 
and established as the base peak and there were no unwanted fragments appearing to cloud the spectra. The fragmentation patterns were unique to each standard including the synthetic cathinones. Methylone and MPDV each showed separate molecular ions as their base peak in addition to other characteristic fragments such as $149,72,56,105 \mathrm{~m} / \mathrm{z}$ (methylone) and 126, 178, 105, and 68m/z (MDPV). Overall the abundance detected for each compound was sacrificed for the molecular information obtained in method 1 and 2 . The default settings in method 1 were selected to proceed with as it remained the quantitatively and qualitatively superior method of the two.

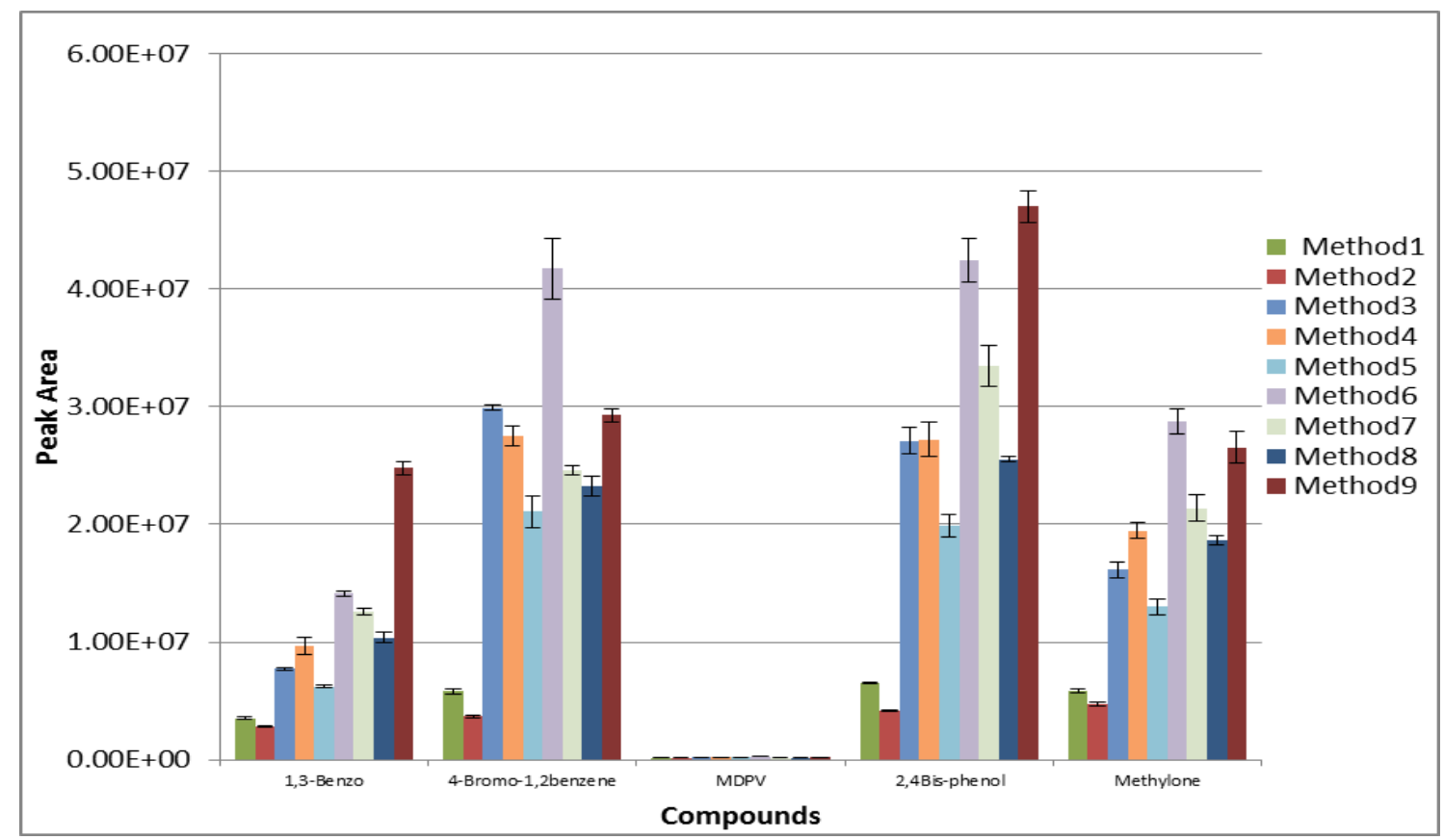

Figure 30.CI-Methanol: Storage Level and Ejection Amplitude Parameter Optimization

From table 17, the complied list of methods created by modifying the default method's background mass were tested (quantitative comparison shown in appendices). By decreasing the background mass of the ionization process there was an increase in 
analyte detection (methods 10 and 12). Although quantitatively sound, method 10 (along with 13 and 14) continuously gave interfering peaks within the chromatogram that spectrally did not belong to any standard. Furthermore, method 12 also suffered from excess ionization of the solvent ran within the sample. The emission of the solvent was so powerful that the solvent to solute peak ratio was severely offset. Although a clear correction from such an occurrence would be to extend the solvent delay on the analytical run. This correction would also result in the loss of fast eluting compounds. To avoid hindering the qualitative collection of any VOCs present, these methods were eliminated.

Methods 1 and 11, simultaneously compared, each resulted in a strong detection for the five analytes. Moreover, these methods also showed similar fragmentation patterns for each analyte. Spectrally there was no additional information that could be produced to determine which method was optimal to proceed forward with. There was also no significant difference in the detection of the standards from one method to the next $(\mathrm{t}=0.11001$; at $\mathrm{p}=0.05)$. Resolution comparison resulted in method 11 experiencing slight tailing for some of the standards. The default settings suggested within the manual were chosen again to proceed after concluding that this method once again gave the most beneficial spectral information among all the other methods. In addition, as there was no advantage from decreasing the background noise level from 55 to $45 \mathrm{~m} / \mathrm{z}$, method 1 was selected. 
Table 17. Chemical Ionization (Methanol) Parameter Modification of Background Mass

\begin{tabular}{|c|c|c|c|c|c|c|}
\hline Method Name & $\begin{array}{c}\text { Storage } \\
\text { Level }\end{array}$ & $\begin{array}{c}\text { Ejection } \\
\text { Amp }\end{array}$ & BM & TIC & $\begin{array}{c}\text { Max } \\
\text { IT }\end{array}$ & $\begin{array}{c}\text { Max } \\
\text { RT }\end{array}$ \\
\hline Methanol Method1 & 19 & 15 & 55 & 5000 & 2000 & 40 \\
\hline Methanol Method10 & 19 & 15 & 50 & 5000 & 2000 & 40 \\
\hline Methanol Method11 & 19 & 15 & 45 & 5000 & 2000 & 40 \\
\hline Methanol Method12 & 19 & 15 & 40 & 5000 & 2000 & 40 \\
\hline Methanol Method13 & 19 & 15 & 60 & 5000 & 2000 & 40 \\
\hline Methanol Method14 & 19 & 15 & 65 & 5000 & 2000 & 40 \\
\hline
\end{tabular}

Assessment on the effect ionization time modification had on the ionization process lead to the elimination of both methods 17 and 18 quantitatively (table 18). Decreasing the ionization time to 1000 and $500 \mu$ s, respectively, both showed difficulty in quantification of the $50 \mathrm{ppm}$ mixture. While automatic integration was not achieved, the manual integration of these peaks would lead to a substantial amount of human error encountered, fluctuating between each trial and peak integrated. Furthermore, systematic limitations established within these methods would prove problematic in the identification of lower extracted volatiles present in a sample's headspace.

Overall the detection limitations can be explained from lowering the ionization time too low. Not allowing for more of the reagent to be ionized resulted in a smaller or limiting number of reagents ions present to interact with the sample. The decreased amount of reagent ions directly correlates to the number of sample ions produced; thus decreasing the number of molecules bearing a positive and detectable. The same occurrence was seen within method 16 for only a few compounds. 
Table 18. Chemical Ionization (Methanol) Parameter Modification of Maximum Ionization Time (Max IT)

\begin{tabular}{|c|c|c|c|c|c|c|}
\hline Method Name & $\begin{array}{c}\text { Storage } \\
\text { Level }\end{array}$ & $\begin{array}{c}\text { Ejection } \\
\text { Amp }\end{array}$ & BM & TIC & $\begin{array}{c}\text { Max } \\
\text { IT }\end{array}$ & $\begin{array}{c}\text { Max } \\
\text { RT }\end{array}$ \\
\hline Methanol Method1 & 19 & 15 & 55 & 5000 & 2000 & 40 \\
\hline Methanol Method15 & 19 & 15 & 55 & 5000 & 2500 & 40 \\
\hline Methanol Method16 & 19 & 15 & 55 & 5000 & 1500 & 40 \\
\hline Methanol Method17 & 19 & 15 & 55 & 5000 & 1000 & 40 \\
\hline Methanol Method18 & 19 & 15 & 55 & 5000 & 500 & 40 \\
\hline
\end{tabular}

Qualitatively comparison of the remaining methods showed similarities. Method 1 had a slight advantage in the resolution and sharpest of the elution of compounds from the column; yet not definite to optimally select this method. Spectral qualitative information obtained from each method was also the same. Every molecular mass of each standard was identified as the base peak and the formation of other unique characteristics fragments were observed for each standard. Quantitative measures were the deciding factors between these methods. Increasing the ionization time to the maximum value allowed for the maximum yield of reagent ions to interact with the sample. This increase resulted in a higher detection yielded for the standards (method 15). Although not significantly different, an increase in sensitivity can be useful for the quantification of smaller emitting compounds into the headspace. Optimally, method 15 was selected to determine the last parameter for chemical ionization using methanol as a reagent.

Once the initial parameters were optimized, the maximum reaction time was varied to determine its effect (table19). The relatively low yield experienced when the reaction time was decreased for methods $23-25$, caused them to be withdrawn. For the remaining methods there was a positive correlation established, as the reaction time increased so did 
the abundance detection for each analyte; with no statistical difference observed.

Preliminary spectral analysis first revealed errors when the reaction times were held at 55 and $60 \mathrm{msec}$. Prolonging the reaction length to these times caused a significant amount of noise present after the molecular mass of each analyte was discovered. As stated before, working with unknown samples can become problematic if the molecular mass identified isn't the correct mass for the unknown species. For this reason these methods were withdrawn from consideration. Overall, method 20 (maximum reaction time of $50 \mathrm{msec}$ ) was selected as the optimal method, considering all parameters being examined. Careful consideration showed the spectrum provided a more clean ionization pattern with minimal background noise in comparison to method 15 and 19. This method also showed a growth in sensitivity without compromising any spectral information that could be obtained.

Table 19.Chemical Ionization (Methanol) Parameter Modification of Maximum Reaction Time (Max RT)

\begin{tabular}{|l|c|c|c|c|c|c|}
\hline Method Name & $\begin{array}{c}\text { Storage } \\
\text { Level }\end{array}$ & $\begin{array}{c}\text { Ejection } \\
\text { Amp }\end{array}$ & BM & TIC & $\begin{array}{c}\text { Max } \\
\text { IT }\end{array}$ & $\begin{array}{c}\text { Max } \\
\text { RT }\end{array}$ \\
\hline Methanol Method15 & 19 & 15 & 55 & 5000 & 2500 & 40 \\
\hline Methanol Method19 & 19 & 15 & 55 & 5000 & 2500 & 45 \\
\hline Methanol Method20 & 19 & 15 & 55 & 5000 & 2500 & 50 \\
\hline Methanol Method21 & 19 & 15 & 55 & 5000 & 2500 & 55 \\
\hline Methanol Method22 & 19 & 15 & 55 & 5000 & 2500 & 60 \\
\hline Methanol Method23 & 19 & 15 & 55 & 5000 & 2500 & 35 \\
\hline Methanol Method24 & 19 & 15 & 55 & 5000 & 2500 & 30 \\
\hline Methanol Method25 & 19 & 15 & 55 & 5000 & 2500 & 25 \\
\hline
\end{tabular}

Acetonitrile was the last reagent the Varian software was equipped to utilize and therefore was tested. As previously done with the prior solvents, the methanol solvent 
was withdrawn from the bulb with a pastuer pipette and dried in the oven overnight. Afterwards the acetonitrile was added to the bulb and connected to the system. Adjustments were made until the gas entering into the mass spectrometer had reached a stabilized and optimal ratio of $42 \mathrm{~m} / \mathrm{z}$ to $41 \mathrm{~m} / \mathrm{z}$. As indicted in table 20 the storage level and ejection amplitude where modified first.

Ionization settings ran within the default method proved to be slightly more sensitive than the modifications or adjustments implemented by the other techniques ( $F$ calculated: 1.33824; f critical: 2.20852). However qualitative analysis failed to identify the molecular ion present for any of the five compounds that were tested. Elimination due to fragmentation severity, the default method was unable to maintain the whole molecular structure for any analyte. In addition to this finding, all methods which employed a storage level of $25 \mathrm{~m} / \mathrm{z}$ were eliminated from selection (method 7-9). Increasing the storage level to a ratio closer to the molecular weight of the reagent proved to be problematic in the detection of methylone. None of these methods were able to detect and quantify the methylone standard and also experienced a decrease in sensitivity for the detection of the second cathinone derivative, MDPV. Methods 7-9 required manual integration for the quantification and only provided a low abundance, thereby deemed unsuitable for successful ionization. 
Table 20.Chemical Ionization (Acetonitrile) Parameter Modification of Storage Level and Ejection Amplitude

\begin{tabular}{|c|c|c|c|c|c|c|}
\hline Method Name & $\begin{array}{c}\text { Storage } \\
\text { Level }\end{array}$ & $\begin{array}{c}\text { Ejection } \\
\text { Amp }\end{array}$ & $\mathbf{B M}$ & TIC & $\begin{array}{c}\text { Max } \\
\text { IT }\end{array}$ & $\begin{array}{c}\text { Max } \\
\text { RT }\end{array}$ \\
\hline Acetonitrile Method1 & 19 & 15 & 65 & 5000 & 2000 & 40 \\
\hline Acetonitrile Method2 & 19 & 18 & 65 & 5000 & 2000 & 40 \\
\hline Acetonitrile Method3 & 19 & 12 & 65 & 5000 & 2000 & 40 \\
\hline Acetonitrile Method4 & 22 & 15 & 65 & 5000 & 2000 & 40 \\
\hline Acetonitrile Method5 & 22 & 18 & 65 & 5000 & 2000 & 40 \\
\hline Acetonitrile Method6 & 22 & 12 & 65 & 5000 & 2000 & 40 \\
\hline Acetonitrile Method7 & 25 & 15 & 65 & 5000 & 2000 & 40 \\
\hline Acetonitrile Method8 & 25 & 18 & 65 & 5000 & 2000 & 40 \\
\hline Acetonitrile Method9 & 25 & 12 & 65 & 5000 & 2000 & 40 \\
\hline
\end{tabular}

By increasing the ejection amplitude from 15 to $18 \mathrm{v}$ and maintaining the default storage setting, there was a significant decrease in the detection of 1,3-Benzodioxole, 4Bromomethylenedioxypriopohenone, and methylone compared to the default method (similar to the other methods). Although quantitatively inferior, qualitatively method 2 resulted in a greater yield of molecular information. Similar to method 2, methods 3-6 resulted in a moderate level of spectral information obtained for the mixture. Within these methods the base peak fluctuated between the molecular mass and smaller fragments that were produced. Methods in this range did not enhance the spectral value beyond what was observed in method 2. For evaluation of the background mass, increasing the amplitude of ejection to $18 \mathrm{v}$ proved ideal for successful ionization.

All modifications of the background mass experienced an observed increase in noise between each run, when using the acetonitrile reagent. From the list of methods experimentally designed methods 13 and 14, which decreased the background mass to 55 and $50 \mathrm{~m} / \mathrm{z}$ respectively, were eliminated first. Both methods were unable to successfully 
quantify any of the cathinone standards. Furthermore, these methods also suffered from significant amount of background noise. All five analytes were not exclusively detected in contrast to the noise level and produced an average $\mathrm{S} / \mathrm{N}$ ratio of less than 32 for each compound. Although more successful in quantification, decreasing the background mass by $5 \mathrm{~m} / \mathrm{z}$, method 12 showed signs of failed ionization. The trials analyzed under this method had a sixth peak eluting at 14.001 minutes with a larger abundance than $60 \%$ of the compounds analyzed within the mixture. This occurrence can be explained by a large amount of interfering components allowed to enter into the ion trap for ionization when the BM was set lower than the default.

Although method 2 contained background noise, the spectra for each compound remained optimal, with the base peak identified as the molecular ion for all expect two. By increasing the background mass to 70 and $75 \mathrm{~m} / \mathrm{z}$ a drop in the unique ions were seen for each compound; as only one fragment was identified, belonging to the molecular ion or base ion, for the analyte. The lack of supporting ions would not sufficiently supplement identification during electron ionization. Therefore method 2 was selected for modification of the ionization time.

Of the drafted methods created to test the functionality of various ionization times, it was noted that by decreasing the maximum time lower than $1500 \mu \mathrm{s}$, there were some concerns when analyzing the spectra for methods 16-18. These methods suffered from chromatographic complications; predominately characterized by an amplitude of unidentifiable background peaks. Within each compound's spectrum there was also a 
significant amount of background that was observed. Although the base peak and molecular ion were identified, any further information could not be distinguished.

Method 15 (ionization time $2500 \mu \mathrm{s}$ ), quantitatively was superior in comparison to the other methods tested. Proven to be nonsignificant, this method provided no additional molecular information beyond what was seen within method 2 . The default ionization time for acetonitrile was successful for efficient ionization to take place. However, by increasing the ionization to the maximum allowed time this allowed for an increase in the abundance of all the known compounds without sacrificing the molecular information obtained. Method 15 was elected to proceed with the configuration of the last parameter.

The modifications of the last parameter were beneficial beyond the default settings of $40 \mathrm{msec}$. The same proportional relationship observed from the modification of both ammonia and methanol was noted when assessing the reaction time for this reagent. If the reaction time was increased or decreased the abundance detected for each analyte would likewise increase or decrease respectively. For this sole purpose, the methods 21 and 22 were eliminated for detection issues. While increasing the reaction time allowed for an increase in the sensitivity of the detector, there was a decrease in each spectrum's fragmentation pattern. Allowing the maximum reaction time to remain constant at 40 msec (along with the previously established parameters) presented method 15 as the optimum ionization method; when the liquid reagent was acetonitrile. 
Through experimentation the optimal settings for each reagent to undergo the chemical ionization process were compared. It was determined that the most suitable method to compliment the electron impact ionization of various synthetic cathinones involved the methanol reagent (table 21). Statistically, this reagent and method resulted in the largest detection of the $50 \mathrm{ppm}$ mixture of standards $(\mathrm{F}$ value $=4.4839$ and $\mathrm{F}$ critical= 3.8852). Within this method the molecular ion was detectable for each standard in addition to unique characterizing fragments as supplementary molecular information. Although each reagent was optimally created to identify the molecular ion, the methanol method was more reproducible and suffered from less interference in comparison. The mass spectra for four of the compounds are highlighted in figure 32. As seen with both the cathinone derivative standards, ionization under electron impact (a) caused severe fragmentation. Both ratios 59 and $126 \mathrm{~m} / \mathrm{z}$ for Methylone and MDPV (respectively) represent only a fraction of the whole molecular structure. Under electron ionization, the identification is proven problematic as suggestive results are determined with very little molecular information to guide the software's library. Ionization by using a chemical reagent has proven beneficial. Not only is the molecular ion identified (b), there are still other surrounding fragments in the spectrum that can be used in tandem with the EI spectrum to select the appropriate compound. With the remaining compounds $(1,3-$ Benzodioxole and 4-Bromo-1,2-(methylenedioxy) benzene) the molecular ion was present initially from analysis conducted in electron impact mode. However, chemical ionization offered a cleaner spectrum in comparison with less background; helping to promote a clear distinguish between all important fragments present. 


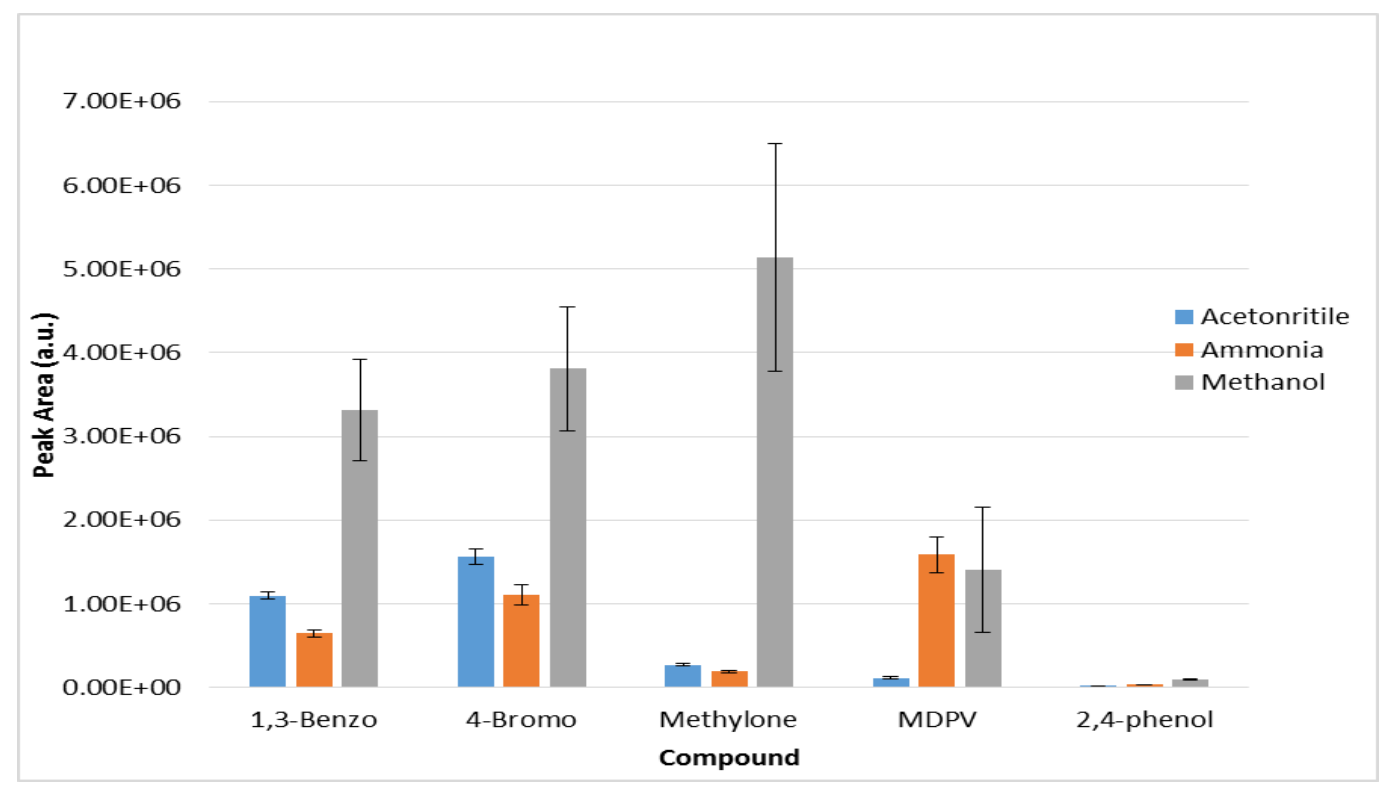

Figure 31. Quantitative Comparison of the Optimal Chemical Ionization Reagent needed for Detection of Synthetic Cathinones

Table 21. Optimum Settings for the Chemical Ionization Method

\begin{tabular}{|l|l|l|l|l|l|l|}
\hline $\begin{array}{l}\text { Method } \\
\text { Reagent }\end{array}$ & $\begin{array}{l}\text { Storage } \\
\text { Level }\end{array}$ & $\begin{array}{l}\text { Ejection } \\
\text { Amplitude }\end{array}$ & $\begin{array}{l}\text { Background } \\
\text { Mass }\end{array}$ & $\begin{array}{l}\text { Target } \\
\text { TIC }\end{array}$ & Max IT & Max RT \\
\hline Methanol & $19 \mathrm{~m} / \mathrm{z}$ & 15 volts & $55 \mathrm{~m} / \mathrm{z}$ & $\begin{array}{l}5000 \\
\text { counts }\end{array}$ & $2000 \mu \mathrm{s}$ & $50 \mathrm{msec}$ \\
\hline
\end{tabular}



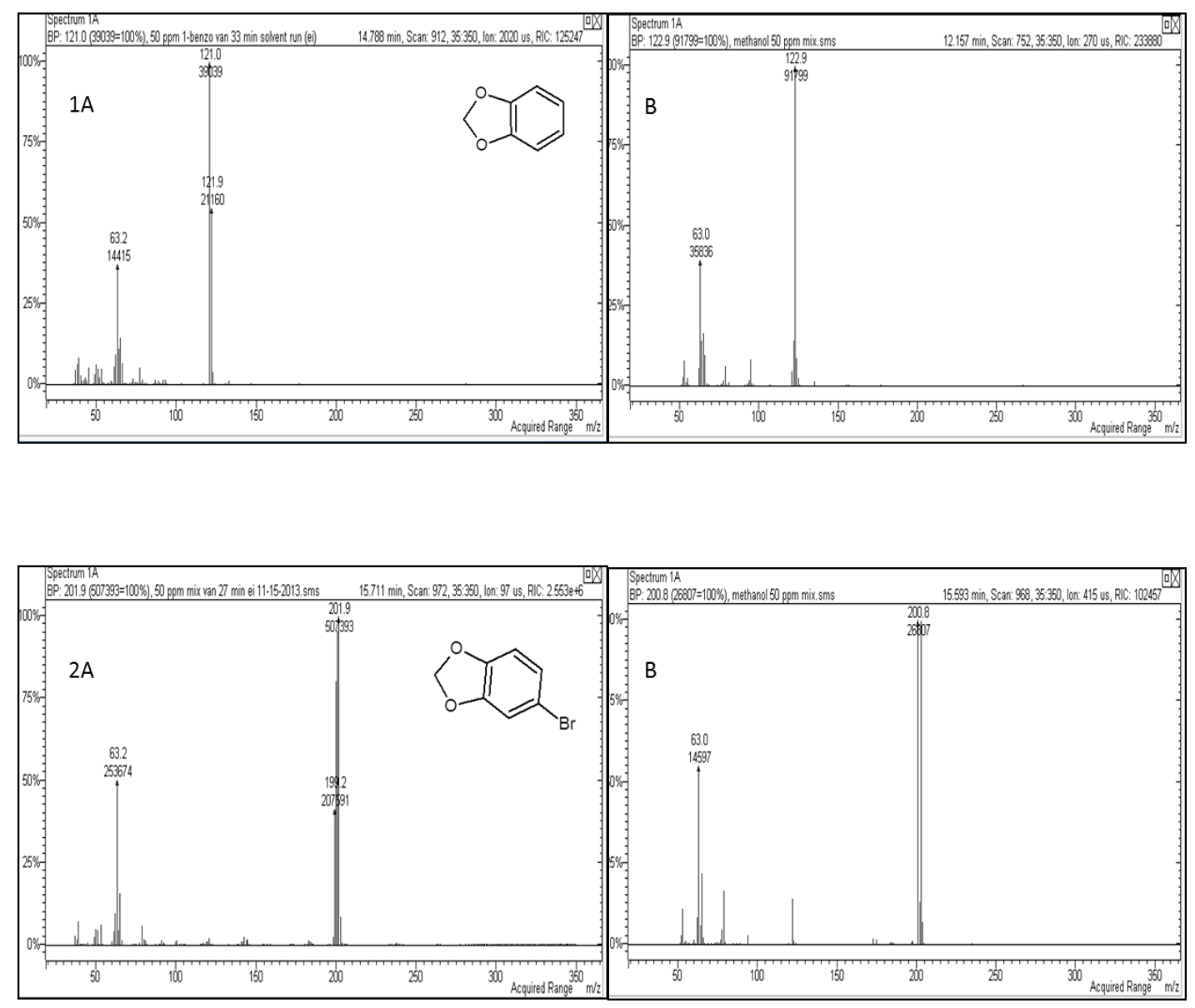

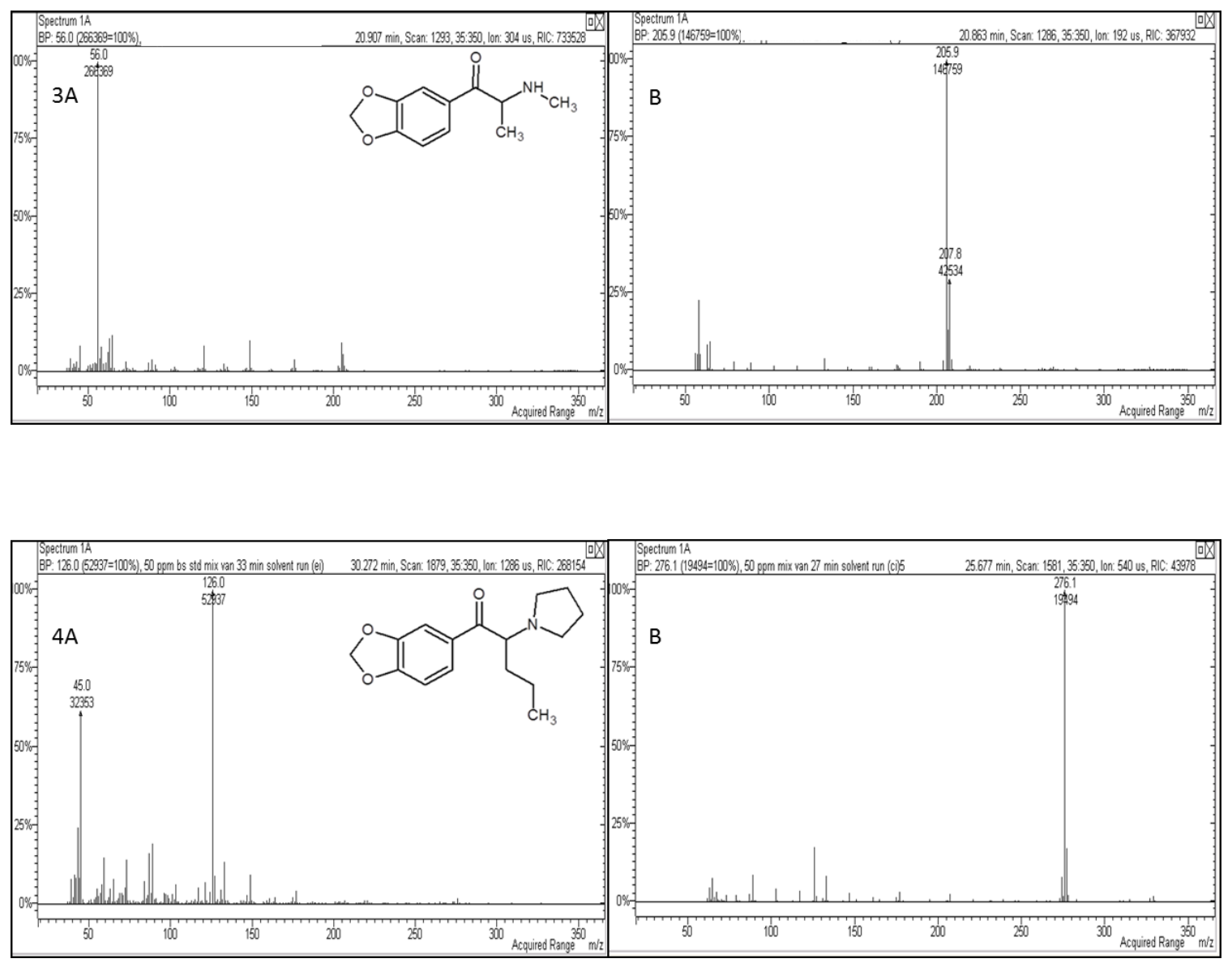

Figure 32. Fragmentation Pattern of Four Compounds Ionized by (a) Electron Impact Mode (b) Chemical Ionization Mode; 1) 1,3-Benzodioxole, 2) 4-

Bromo(methylenedioxy)benzene, 3) Methylone, 4) MDPV

The chemical ionization method developed will be used alongside the electron ionization to provide additional molecular information that may be lost during excessive ionization. 


\subsection{HS-SPME Method Optimization for the Detection of Volatile Organic Compounds Released from Synthetic Cathinones}

\subsubsection{Optimal Fiber Selection for Headspace Solid-Phase Microextraction (HS-SPME)}

Although successfully used prior to this study, due to the novel approach of conducting a headspace extraction method for the odor profiling of synthetic cathinones, the most suitable fiber needed to be identified first. The SPME fibers evaluated during this task were $75 \mu \mathrm{m}$ CAR/PDMS, $65 \mu \mathrm{m}$ PDMS/DVB, 50/30 $\mu \mathrm{m}$ DVB/CAR/PDMS, and $85 \mu \mathrm{m}$ Polyacrylate (PA). These fibers were compared by their efficiency and ability to extract an abundance of compounds.

The sampling conditions expressed in section 4.2 were used during this study. The fibers were allocated an hour for analyte extraction and the results were compared in figure 33. The polyacrylate fiber was excluded as the extraction capability for this fiber was approximately $2 \%$ of the total number of compounds identified. Qualitatively this fiber was only able to detect one compound, requiring manual integration, and therefore was rejected. Analysis of the remaining SPME fibers concluded that the most beneficial polymer chemistry for volatile extraction of synthetic cathinones was polydimethylsiloxane/divinylbenzene (PDMS/DVB). The overall percentage of compounds identified by the PDMS/DVB fiber was approximately $78 \%$ in comparison to the CAR/PDMS and DVB/CAR/PDMS fibers which extracted $65 \%$ and $57 \%$ of all compounds noted, respectively. The PDMS/DVB fiber exceled in overall compound abundance detected throughout the sample in comparison to the remaining three fibers, while yielding a lower relative error. The characteristics of compounds obtained from the 
PDMS/DVB fiber included nitrogen groups, ketones, and various elongated and branched alkane chains. These functional groups are also found in the backbone structure for the cathinone derivatives.

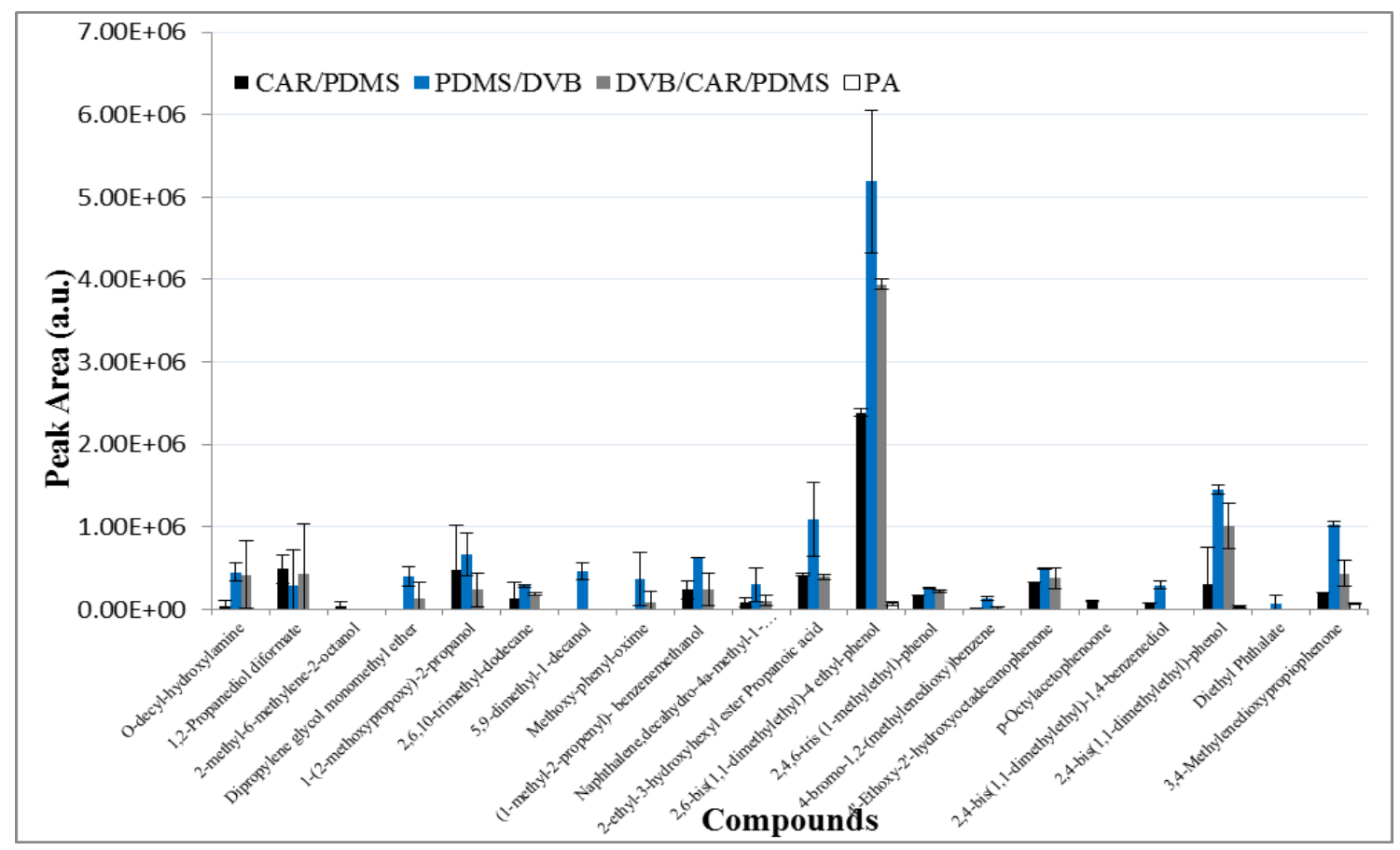

Figure 33. HS-SPME Fiber Chemistry Comparison of the Extraction of the Target Compounds Potentially Associated with Synthetic Cathinones

\subsubsection{Optimal Extraction Parameters for HS-SPME}

As discussed in section 4.2.3, there are several parameters which promote the interaction between a fiber and volatile compounds. Before examining these parameters, assessment of whether a temperature beyond room setting would be beneficial was tested. Although initial testing with the synthetic cathinones was performed at room temperature, literature does highlight the benefit of extraction conducted under elevated temperatures. 
The extraction process of 1 hour equilibrium and extraction was performed on the methylone case. The extraction conducted without the application of heat (figure 34) was successful in yielding approximately eleven compounds within the headspace of the drug. The extraction performed at $50^{\circ} \mathrm{C}$ was selected as an ideal starting point based on the literature settings of extractions performed on methamphetamine and MDMA.

Structurally similar to the drugs, it was suggested that synthetic cathinones would be able to tolerate the setting. A heating temperature of $50^{\circ} \mathrm{C}$ was also selected as it was approximately twice as high as room temperature and therefore theoretically should increase the number of compounds seen. As depicted by figure 34, increasing the extraction temperature did in fact increase the number of compounds obtained in comparison to when the volatile collection was performed at room temperature. In total the PDMS/DVB fiber was able to extract 24 compounds while only losing a small fraction of the volatiles seen at the lower temperature (less than 19\%). The compounds observed under the extraction performed at room temperature were predominately characterized by various alcohols. While performing the initial extraction under the application of heat promoted the extraction of alcohols in addition to ketones, longer alkane chains and various amines were also noted; therefore heat was applied to all samples. 


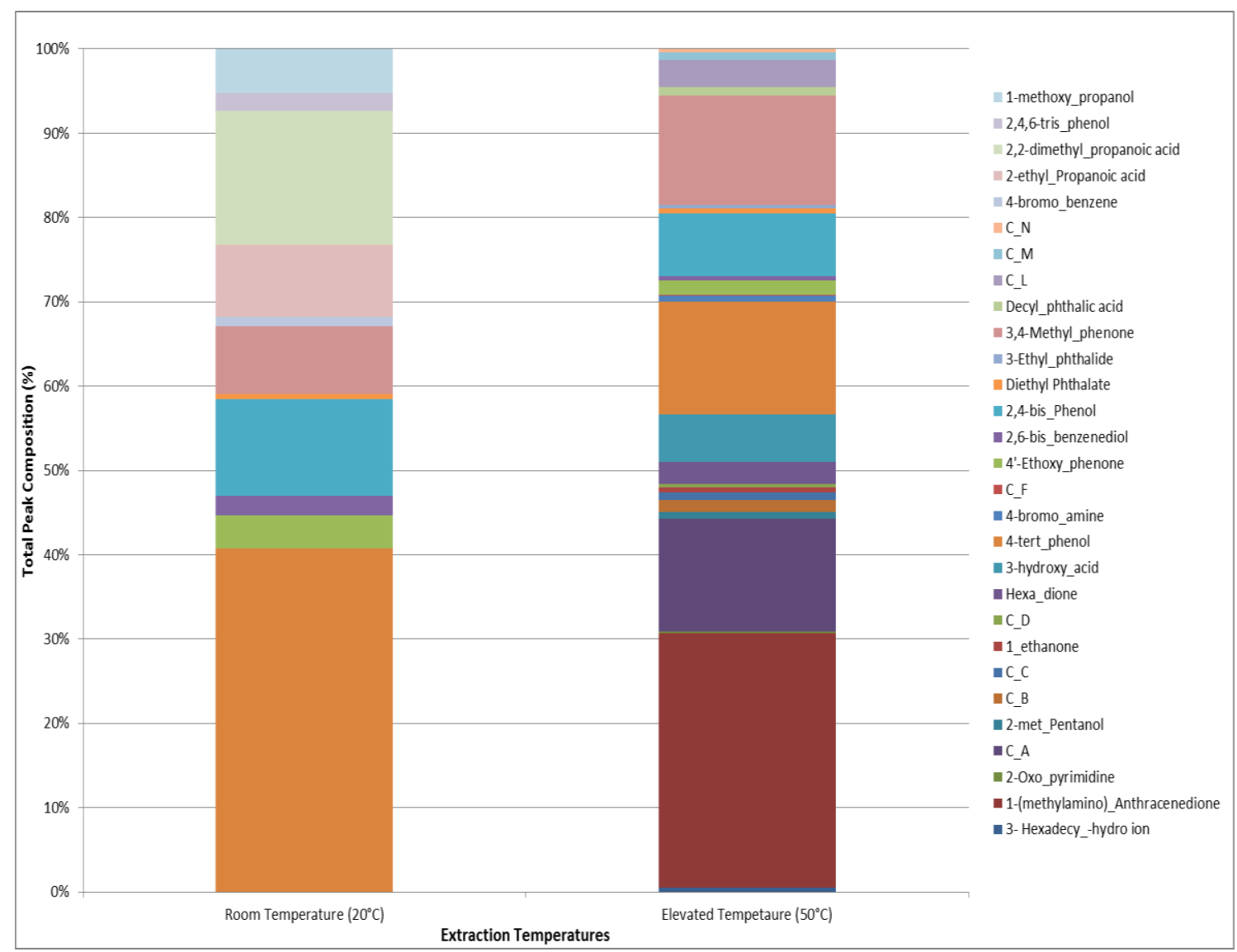

Figure 34. Headspace Extraction Collected at Room Temperature vs an Elevated Temperature $\left(50^{\circ} \mathrm{C}\right)$

Establishing the length of time required for the adsorption or absorption of any volatile onto a fiber, requires the determination of exposure length to maximize the polymer's interaction with any volatile compound. To test the appropriate length of exposure, the times were focused between 1-6 hours for several reasons. Preliminary extractions initially focused on an hour of exposure. Two factors determined the direction and length of variation for testing the fiber exposure. The first factor was the length of time permitted to complete the extraction process. Headspace collection of the volatile compounds emitted from the sample would take place in the crime lab or facility in 
which the drug was housed. Analyzing impure samples confiscated by law enforcement required that all analysis be performed on the premises of the agency. The time frame of the fiber exposure had to beginning and end within a reasonable time frame before the facility locked down. The working hours of any criminalist or analyst are 8 hours so the set up and exposure had to be completed within that time frame. The second factor was the error produced when extractions were held longer than 8 hours (depletion of sample). These criterions led to $1,2,4$, and 6 hours being selected as the variable exposure times tested.

The initial observations of reoccurring compounds across various extraction times were proven to be a good indication of the volatiles generated within the chromatograms. The lowest time frames analyzed ( 1 and 2 hours) were eliminated first. Analyzing the profile extracted from the methylone case revealed that a large number of the compounds within the chromatogram were from column breakdown. Siloxane peaks observed during both extraction times can be the result of column breakdown naturally occurring that bears a similar signal to noise ratio to various analytes. These peaks can also be the result of slight breakdown within the PDMS/DVB fiber. Overall the abundance of the compounds detected was not high enough to overcome the significant noise contributed to background factors. With a limited number of compounds detected and quantified, these extractions were unsuccessful at contributing to the identification of the odor profiles of synthetic cathinones.

Increasing from 2 to 4 hours of exposure time was the next step in determining if an extended period of exposure would allow ample time to identify any volatile 
compounds. In addition, looking at 6 hours was also examined. Exposure for 6 hours proved to be the most abundant for the quantifiable extracted analytes. This could be expected, as increasing the exposure permits an increase in the rate of absorption/adsorption between the compounds and fiber chemistry. Full analysis of this time revealed that there were four compounds predominately extracted from the headspace. By increasing the fiber exposure to 6 hours (figure 35) the fiber was able to extract a large amount of 3,4-methylenedioxypropiophenone, which accounted for majority of the headspace extracted. By increasing the exposure, these four compounds increased their extraction by $85.63 \%$ in comparison to the amount extracted by four hours. However, while there was an increase in theses compounds' extraction, there was also a decrease in the amount of the other compounds extracted. 1,3-Benzodioxole-5-(5keto-pentaonic acid, the second largest compound extracted during this time frame, experienced a $50 \%$ decrease in abundance, compared to the compound's presence in the four hour extraction. The same phenomenon occurred with 2,4-bis (1,1-dimethylethyl)phenol (third most extracted compound) which showed an decrease in the extracted quantity from the 2 and 4 to 6 hour exposure time.

Although prolonging the length of exposure from 4 to 6 hours proved quantifiably beneficial, the headspace extracted predominately consisted of one compound (whose binding affinity seemed to increase over time). A significant number of the compounds detected in the four hour extraction were lost. This decrease in overall molecular collection can be explained by the fact that a exposure length beyond 4 hours would allow several compounds, initially extracted, the chance to desorbed off the fiber; allowing more of the 3,4-methylenedioxypropiophenone to bind. In addition, any other 
extracted compound could have reached the limit of detection and quantification within the gas chromatogram and mass spectrometer. For these reasons it was also concluded that the exposure time of 6 hours would not optimally succeed in the identification of the synthetic cathinones' odor profile.

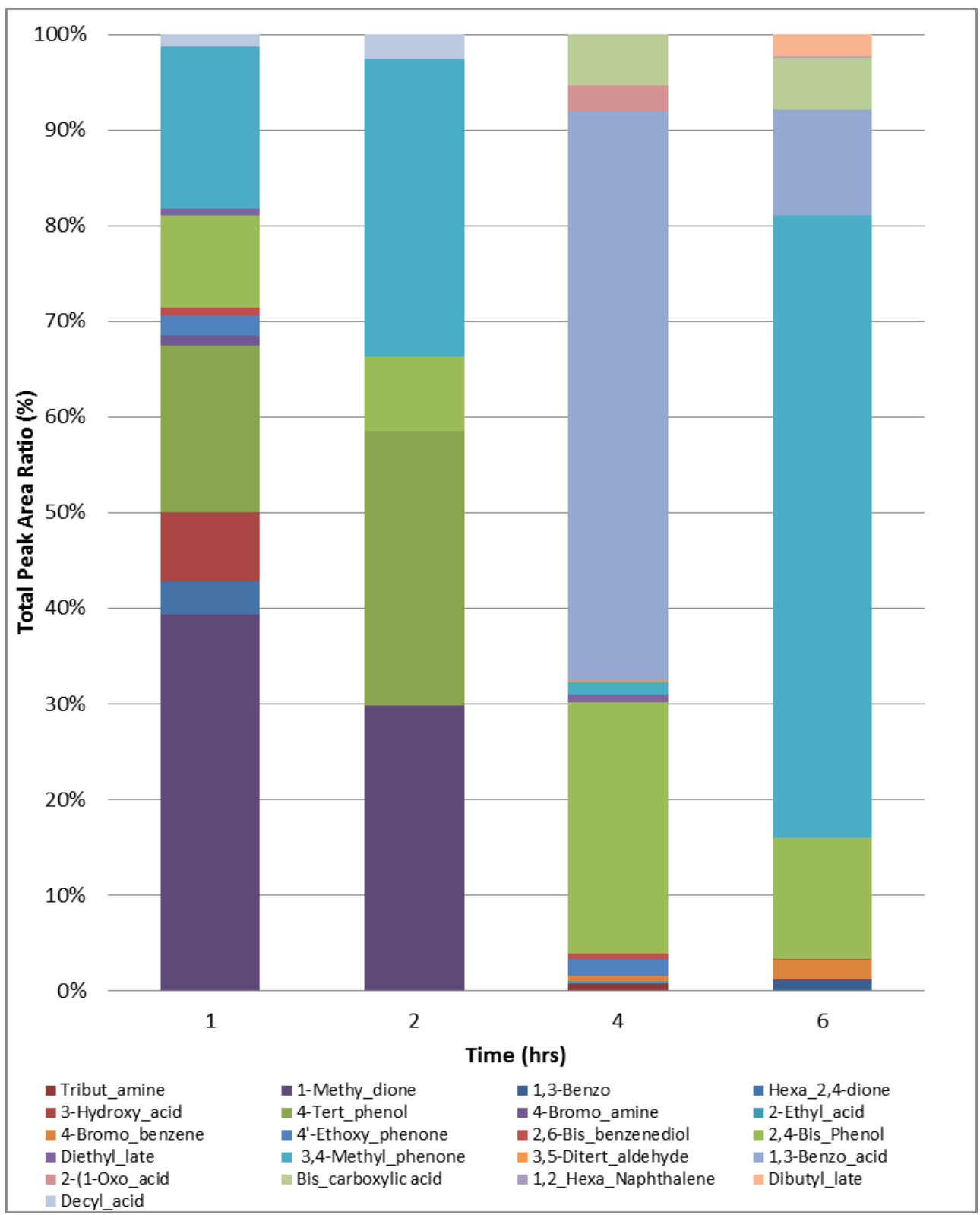

Figure 35. Visual Observation on the Variation of Extractions Times and its Effect on the Collection of VOCs 
Table 22. Scientific Names for the Compounds Discovered in the Extraction Process (Figure 35)

\begin{tabular}{|c|l|}
\hline Abbreviation & \multicolumn{1}{c|}{ Scientific Name } \\
\hline Tribut_amine & Tributylamine \\
\hline 3-Hydroxy_acid & $\begin{array}{l}\text { 3-Hydroxy-2,4,4-trimethylpentyl ester-2-methyl-propanoic } \\
\text { acid }\end{array}$ \\
\hline Bis_carboxylic acid & $\begin{array}{l}\text { Bis (2-methylpropyl) ester 1,2-benzenedioxycarboxylic } \\
\text { acid }\end{array}$ \\
\hline 1,3-Benzo_acid & 1,3-Benzodioxole-5-(5 keto-pentanoic acid \\
\hline 3,4-Methyl_phenone & 3,4-Methylenedioxypropiophenone \\
\hline Dibutyl_late & Dibutyl Phthalate \\
\hline 1-Methy_dione & $\begin{array}{l}\text { 1-Methy_dione: 1-(methylamino)-4-[(4-methylphenyl) } \\
\text { amino]-9,10- Anthracenedione }\end{array}$ \\
\hline 2-(1-Oxo_acid & 2-(1-oxopropyl) Benzoic acid \\
\hline 3,5-Ditert_aldehyde & 3,5-Ditert-Butyl-4-hydro-benzaldehyde \\
\hline 4'-Ethoxy_phenone & 4'-Ethoxy-2'-hydroxyacetophenone \\
\hline 4-Bromo_amine & 4-Bromo-N,N-dimethyl-benzenamine \\
\hline Diethyl_late & Diethyl Phthalate \\
\hline Decyl_acid & Decyl isobutyl ester phthalic acid \\
\hline Hexa_2,4-dione & $\begin{array}{l}\text { Hexahydro-3,6-dimethyl-1-(40morpholinobutyl)- } \\
\text { pyrimidine-2,4-dione }\end{array}$ \\
\hline 2,6-Bis_benzenediol & 2,6-Bis(1,1-dimethylethyl)-1,4-benzenediol \\
\hline 2,4-Bis_Phenol & 2,4-Bis (1,1-dimethylethyl)-Phenol \\
\hline 4-Bromo_benzene & 4-Bromo-1,2-(methylenedioxy)-benzene \\
\hline 2-Ethyl_acid & 2-Ethyl-3-hydroxyhexyl ester-2-methyl propanoic acid \\
\hline 1,3-Benzo & 1,3 Benzodioxole \\
\hline
\end{tabular}

A fiber exposure time of 4 hours was selected as the optimal length to proceed forward with. This extraction time proved superior over the other exposure times for several reasons. During this extraction the compounds positively identified in the first two extraction times were also identified within the four hour exposure. This extraction time didn't suffer from a significant amount of background noise, making detection challenging. In comparison to the six hour extraction, there was no loss of compounds witnessed within this headspace. Although there were compounds predominately identified within this time, other surrounding compounds were still detectable and 
quantifiable by the software. Allowing the fiber a total exposure time of 4 hours proved to be better for qualitative and quantitative analysis, with higher reproducibility. Statistically comparing the total abundance extracted from 6 hours to 4 , proved that there was no significant difference ( $\mathrm{t}$-test; $\mathrm{p}=0.22549)$. By overcoming all the disadvantages encountered from the other time frames, 4 hours was optimally used to develop the odor profiles of several synthetic cathinones.

The equilibrium phased was examined to establish the time required for the volatilization reaction to become stabilized. In essence, this determined the time needed for the rate at which the compounds found within the sample to enter into the gas phase (headspace) and settled back into the sample. From preliminary experiments conducted the equilibrium was held for 1 hour for completion. Variations from this time were tested to determine if equilibration within the sample and container could be established in less time or required an extension beyond the hour. The time frames examined within this study included 15 and 30 minutes and 1,2, and 4 hours. After holding the equilibration for an hour the time was modified in opposing directions (30 minutes and 2 hours), tested, and continuously proceed until an equilibration time of 15 minutes and 4 hours were also examined.

As depicted in figure 36, the quantification of each time was compared. Each equilibration test was performed on a concentrated amount (50 ppm) of five mixed standards. By increasing the equilibration to 2 hours and decreasing to 30 minutes, both times displayed a growth in the amount of compounds extracted. Thereafter the length was also increased to four hours (decreased to fifteen minutes) to see if this trend 
continued. By extending the equilibration time to 4 hours there was no visual increase in the amount of compounds extracted. However, decreasing to 15 minutes as shown in figure 36, did provide an increase in the quantification. Although visually there was no exaggerated difference between the equilibration times, statistic testing was performed. ANOVA testing on the extractions performed supported the null hypothesis that the range of equilibrations times, from 15 minutes to 4 hours, were similar $(F=0.127$; $\mathrm{f}$ critical $=$ 2.209). The test showed that by increasing the equilibration time beyond 15 minutes there was no significant change in the amount of analyte extracted from the sample. Therefore, the amount of time needed for the sample's volatilization rate in the forward and reverse direction to stabilize was fifteen minutes and was optimally selected to proceed.

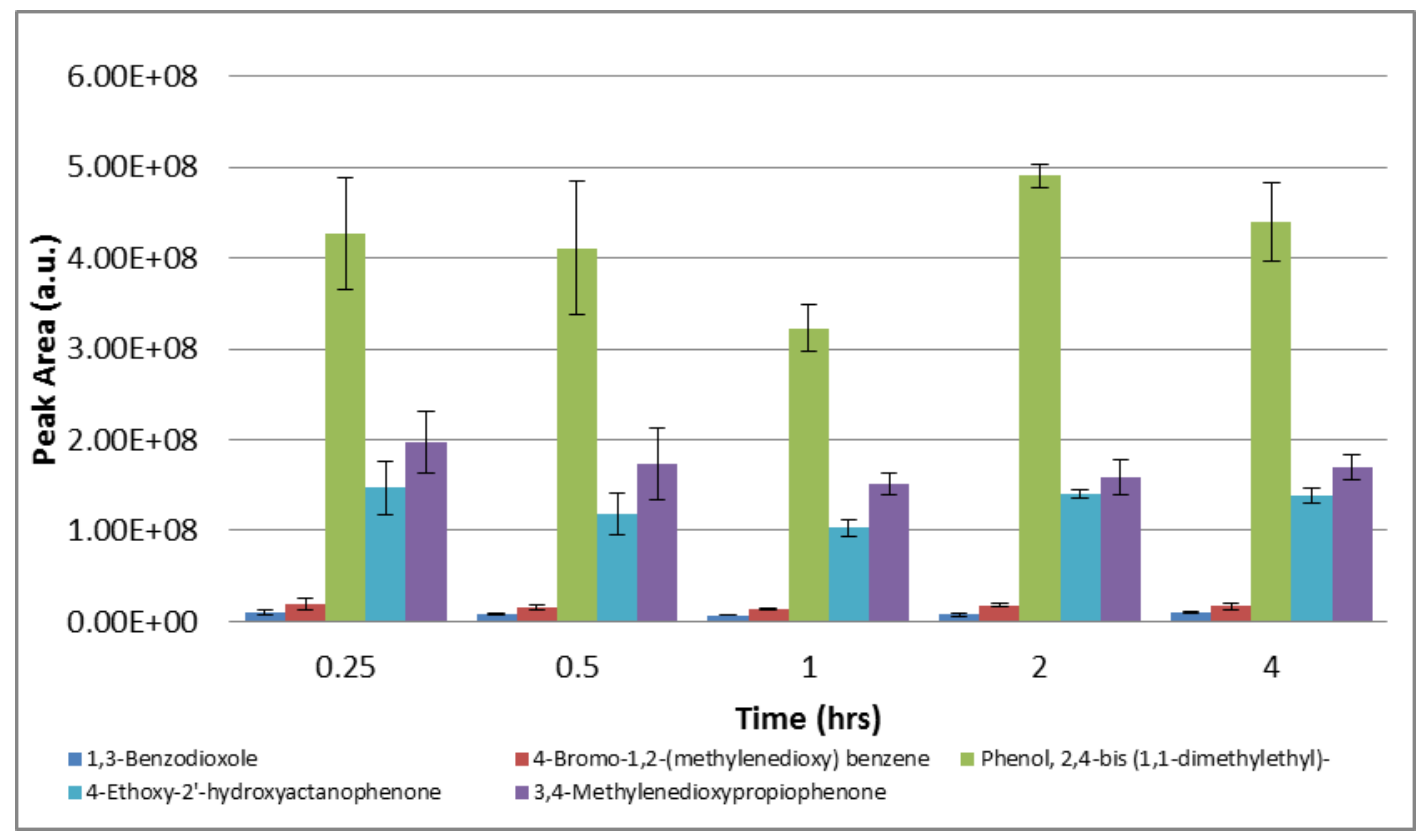

Figure 36. Variation of Times to Establish Equilibrium within a given Volume for Extraction 
After the successful application of heat to extract a larger range of compounds in comparison to room temperature, assessment of the range of temperature was performed. Heating a sample not only promotes a compound's transition in the gas phase but also assist with the absorption between the fiber and VOCs. Various studies conducted which focused on the application of heat, have found that positive extraction can be achieved from ranging the heat settings from $25^{\circ} \mathrm{C}-95^{\circ} \mathrm{C}$; depending on the analyte and its sensitivity. The assessment of heat on a synthetic cathinone sample extracted was tested from a range of $45-60^{\circ} \mathrm{C}$ (in five degree increments).

The temperatures were not examined beyond $60^{\circ} \mathrm{C}$ as a precaution to sample utilized. Working with confiscated samples located in the crime lab, both classified as closed and open/pending cases, sample conservation was essential. The temperature was not decreased lower than $45^{\circ} \mathrm{C}$ as settings below this point failed to produce any benefits beyond what was acquired by the extraction commenced at room temperature. The settings tested were kept in close proximity to $50^{\circ} \mathrm{C}$ and were assessed on two factors: sample degradation and mixture detection. The extractions performed at varying temperatures were examined to detect if there was a proportional relationship established between the temperature and abundance extracted, and which achieved the highest yield. In addition, the extractions were also tested to detect any degradation between the samples that could possibly take place. Initially the study applied $50^{\circ} \mathrm{C}$ to the methylone case to determine whether a change in temperature would cause any breakdown or material loss. Afterwards the parameter conditions were repeated once again with the stock solution created. 
As depicted in figure 37, the extractions performed at various temperatures were visually compared and concluded that $50^{\circ} \mathrm{C}$ resulted in the largest abundance observed for the volatiles. Extractions performed at temperatures beyond $50^{\circ} \mathrm{C}$ experienced a decrease in the quantification of the $50 \mathrm{ppm}$ mixture. Theoretically the decrease in abundance seen with the higher temperatures results from an excess amount of energy provided to the compounds in the drug's composition.

During the collection phase, compounds are extracted from the headspace by absorbing/adsorbing to the fiber's coated chemistry. By applying heat, the amount of compounds available within the headspace increased as the higher temperature enhanced bound breakage from the sample. Once bound to the fiber's chemistry, the excess energy (provided by the increased temperatures) allowed these compounds to desorb from the coated fiber back into the headspace. At temperatures beyond $50^{\circ} \mathrm{C}$, the decrease in abundance detected for each compound was the result of diminished retention and sample breakdown. Temperatures lower than $50^{\circ} \mathrm{C}$ also experienced a decrease in compound retention. This phenomenon occurred as there was not a sufficient amount of energy provided to promote the compounds into the headspace in comparison to the $50^{\circ} \mathrm{C}$ extraction. This temperature was able to extracted a larger abundance and avoid degradation of the sample. 


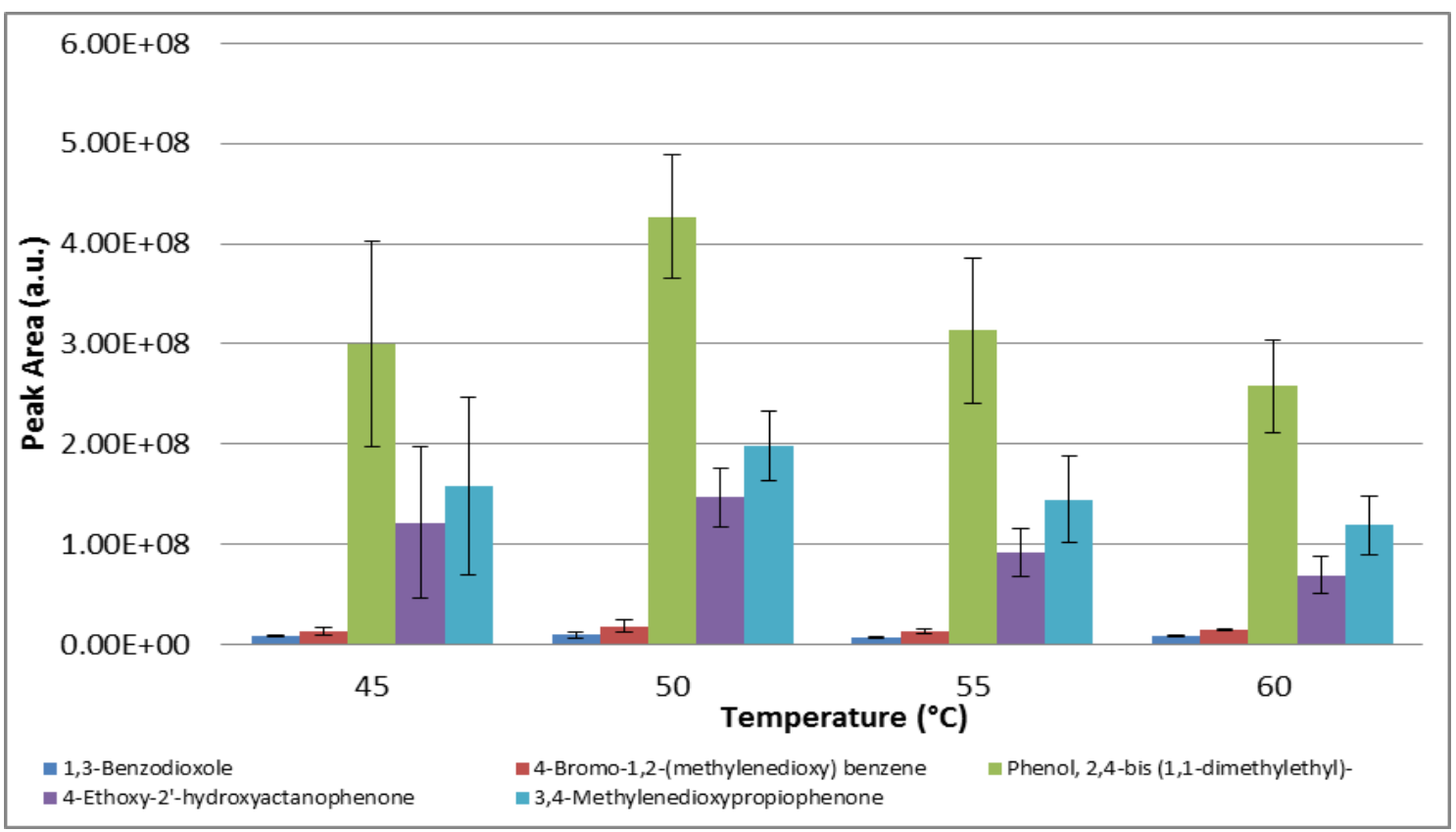

Figure 37. Variation of Temperatures for the Optimal Promotion of VOC Collection

Selection of the extraction temperature was the last HS-SPME extraction parameter chosen. Once ideal factors were determined for the extraction, the desorption process within the GC inlet were measured. The inlet temperature during the fiber exposure was $250^{\circ} \mathrm{C}$. The recommend operating temperature was suggested by Supleco to be maintained between $200^{\circ} \mathrm{C}-270^{\circ} \mathrm{C}$. As $250^{\circ} \mathrm{C}$ was the conditioning temperature for the PDMS/DVB fiber, this temperature was chosen to ensure desorption of the collected analyte. The length of the fiber exposure in the inlet was tested. The desorption time was selected based on how much of the extracted compounds were detection in addition to how much of the extracted compounds remained on the fibers after the exposure. The fiber's residual compounds were observed by undergoing a cleaning session. A cleaning session was conducted by taking the pre-exposed fiber and exposing it into the inlet again 
for an additional five minutes. Each cleaning run was analyzed to detect the amount of residual compounds desorbed. Each fiber underwent a cleaning session until the compounds were no longer present.

The average abundance detected from each inlet exposure time was seen in figure 38. The five minute desorption time was the first eliminated as this length of exposure did not allow for the complete desorption of all the compounds. As a result, this time experienced the lowest detection of all the analytes when compared. By increasing desorption to 10,12, and 15 minutes, the abundance of all the mixed components resulted in an increase as well. Overall, by exposing the fiber into the GC inlet for 15 minutes, the largest detection was achieved. Although this time also resulted in the lowest average RSD (approximately 10\%) per compound, there was no significant difference in the detection between the three remaining times (ANOVA, F calculated: 0.103 and $f$ critical: 3.885). 


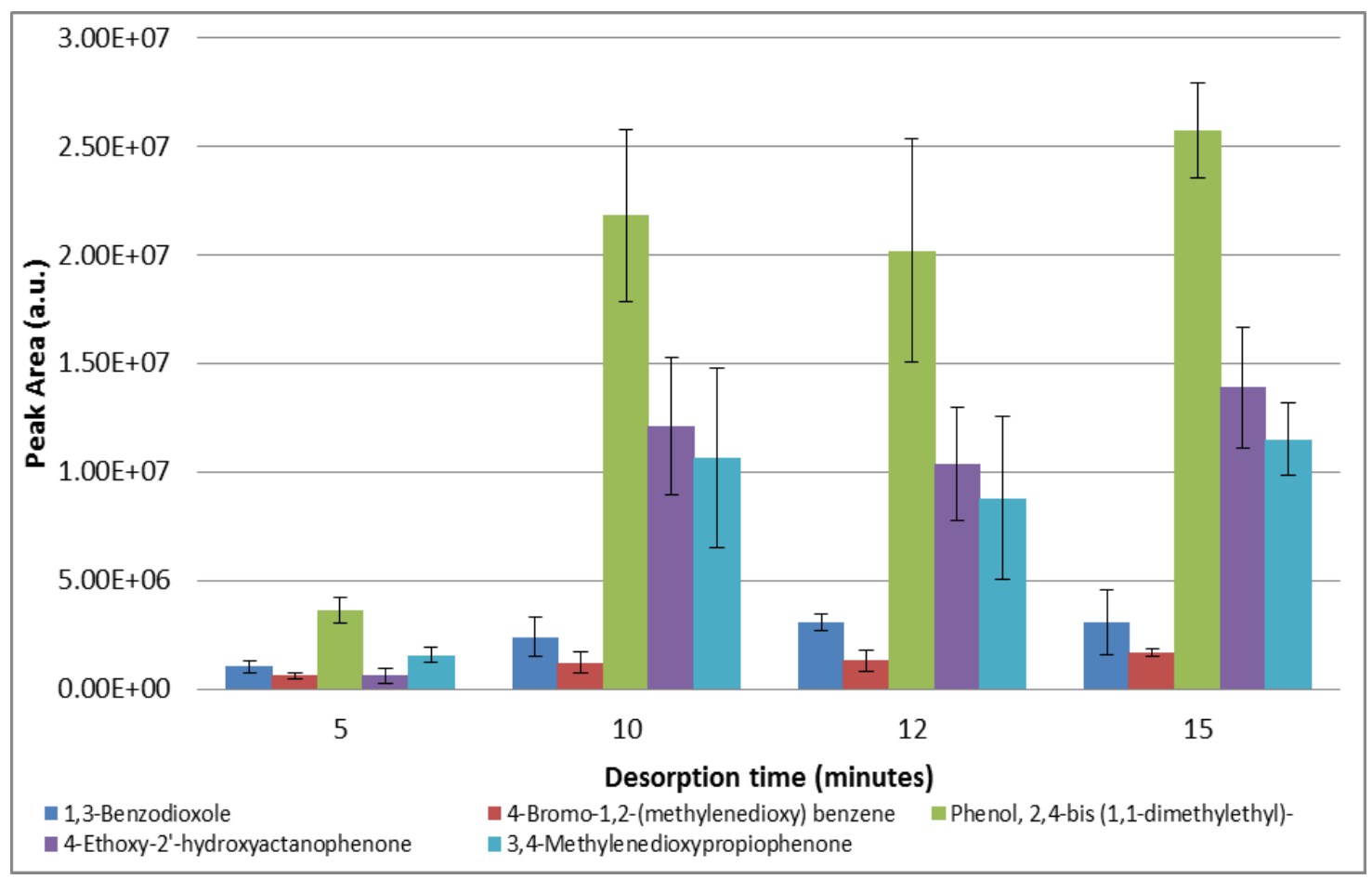

Figure 38. Variation of GC Inlet Exposure for the Desorption of all Extracted VOCs

Determination of the most optimal exposure time for desorption was concluded by examining the carryover (figure 39). As initially expected, the five minute exposure time proved to be the least beneficial as there was a large about of carryover experienced within the first cleaning session. Fibers that were exposed for this time frame required two additional cleans before the absent of any residual compounds. During the examination of the fibers initially exposed for 10 minutes, there were four compounds out of the five in the mixture that were detected again. During the second clean these compounds (with the exception of 2,4-bis (1,1-dimethylethyl)- phenol) were no longer present. The presence of 2,4-bis (1,1-dimethylethyl)- phenol during the second clean was 
only noted in one fiber and can be summed up as the results on a strong affinity to this fiber, requiring a longer exposure.

Continuing the trend, 12 minutes, although not significantly different from 15 minutes in the initial quantification, also experienced carryover observed during cleaning. During the first cleaning session $60 \%$ of the compounds were retained on the fiber. Assessment of the second clean revealed that there was no longer any carryover. The last session undergone for the carryover study was fifteen minutes. Examination of the first clean revealed that all of the compounds initially collected during the extraction process were able to desorb from the fibers given 15 minutes in the GC inlet.

The carryover study supported the theory that every compound has a different binding affinity to the PDMS/DVB fiber, which determined how successful the compound will be at binding to the fiber as well as how strong that bond is. Compounds with a higher affinity required an extended period of time for the desorption process to complete in comparison to the weaker compounds. The cleaning sessions showed that although there was not a significant difference in the abundance detected between the various desorption times, the 15 minutes exposure was the only time frame that did not experience any carryover from the previous run. This desorption time was selected on those premises. Any other time would cause carryover resulting in a false skew in the detection of compounds during continued extractions at a later time. In addition residual compounds can falsely contribute to the profile of any unknown synthetic case examined. 


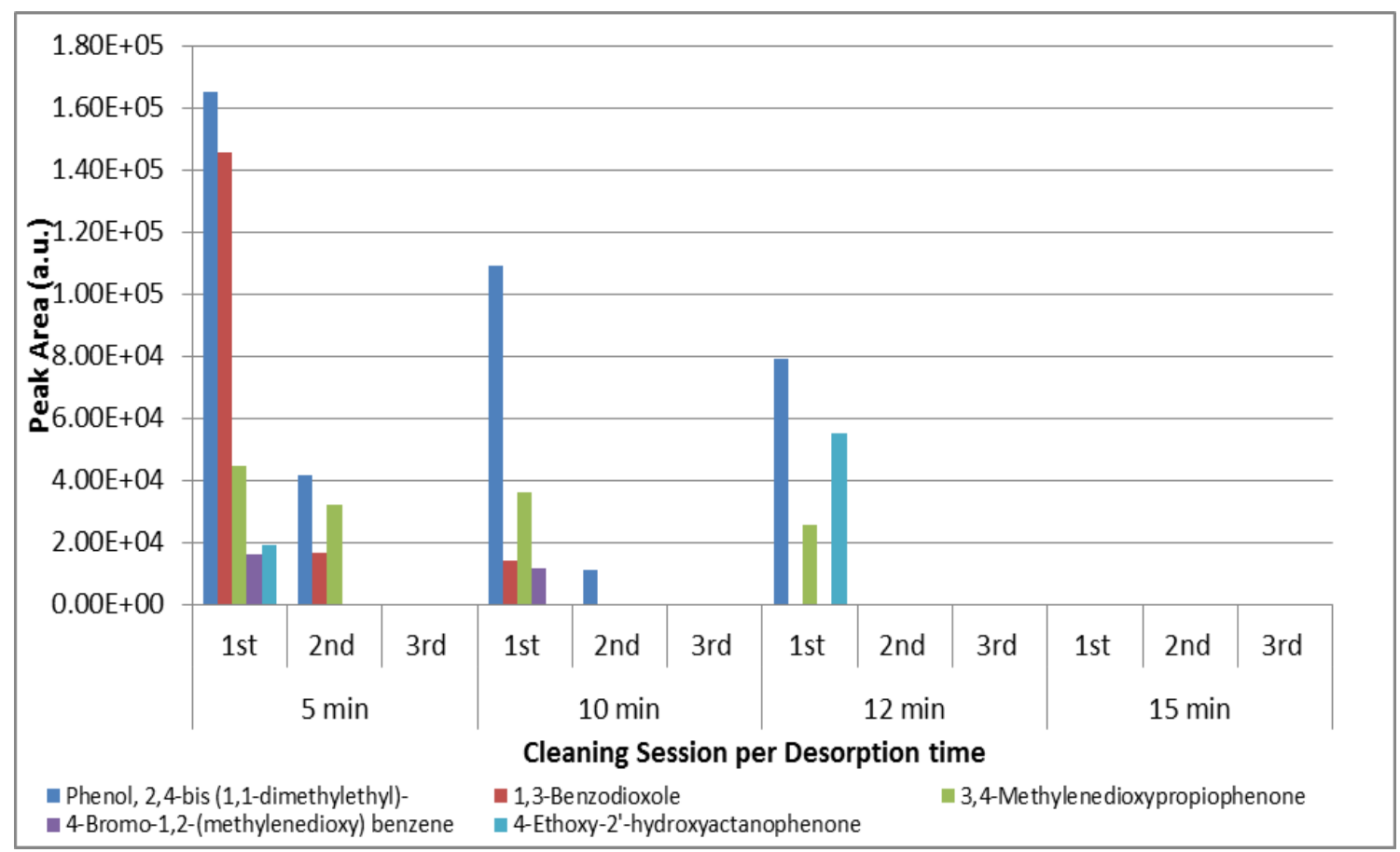

Figure 39. Assessment of the Analytes Retained on the PDMS/DVB fiber after the Initial Desorption

Determination of the desorption length was the last parameter examined for the completion of a headspace extraction method. The parameters determined to be ideal for the development and identification of the odor profile associated with synthetic cathinone are summarized in table 23. 
Table 23. Optimum Headspace-Solid Phase Microextraction Method for the Collection of VOCs from Synthetic Cathinones

\begin{tabular}{|c|c|}
\hline Parameter & Setting \\
\hline Ionization Modes & $\begin{array}{c}\text { Primary: Electron } \\
\text { Complimentary: Chemical }\end{array}$ \\
\hline Fiber Chemistry & Polymethylsiloxane/Divinylbezene \\
\hline Extraction Temperature & $50^{\circ} \mathrm{C}$ \\
\hline Equilibration Time & 15 minutes \\
\hline Fiber Exposure Length & 4 hours \\
\hline GC Inlet Desorption Length & 15 minutes \\
\hline
\end{tabular}

\subsection{Assess the Volatile Organic Compound(s) Released from Synthetic Cathinones}

In total the optimized method was applied to sixteen different synthetic cathinone cases for collection of any volatiles identifiable within the headspace. Each case underwent visual observation, preliminary and standardized GC/MS testing as the following:

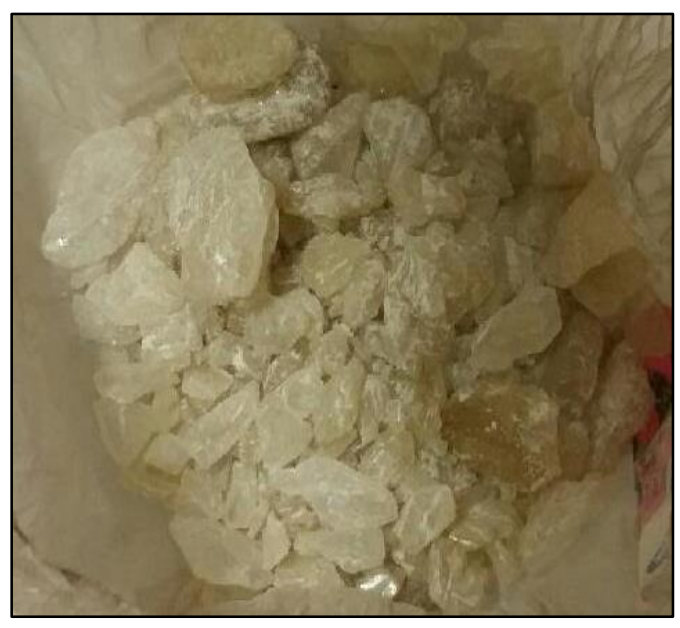

Case 1648297: 22 grams of a white crystalline substance was detained from a suspect. The substance was housed in a brown plastic bag and contained powder and crystalline rock structures. RAMAN was performed as a preliminary test and concluded that the material

Figure 40. Visualization of Case 1 
possibly contained Methylone.

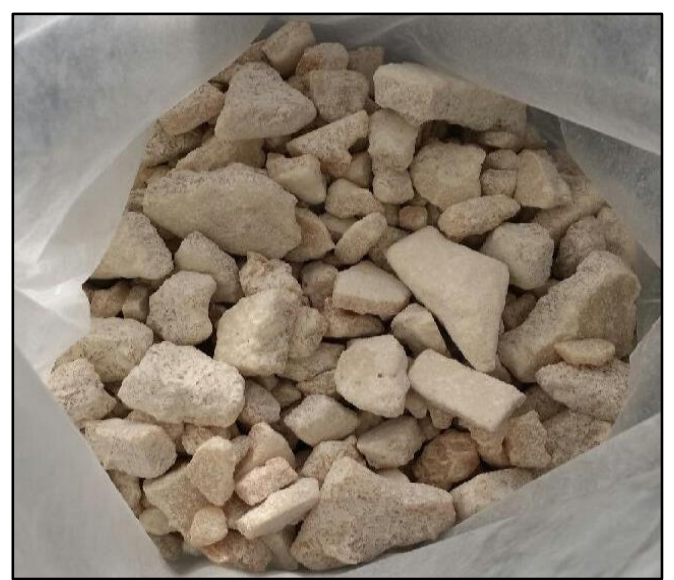

Figure 41. Visualization of Case 2

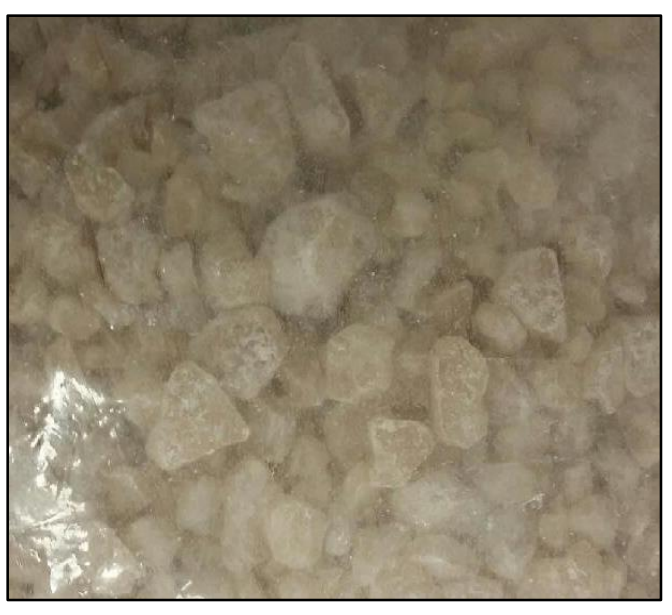

Figure 42. Visualization of Case 3 possibly contained Ethylone.

Case 1796121: 1,046 grams of a substance was confiscated in a clear plastic Ziploc bag. The substance contained a rock formation of various sizes and possessed an off-white color with dark brown specs. RAMAN was performed as a preliminary test and concluded that the material possibly contained Ethylone.

Case 1796122: A clear plastic bag containing 1,134 grams of a substance was seized inbound from a China distributor. The substance contained a rock formation of various sizes. The rocks had a consistency similar to compressed crystals and were slightly transparent; which off-white color. RAMAN was performed as a presumptive test and concluded that the material 


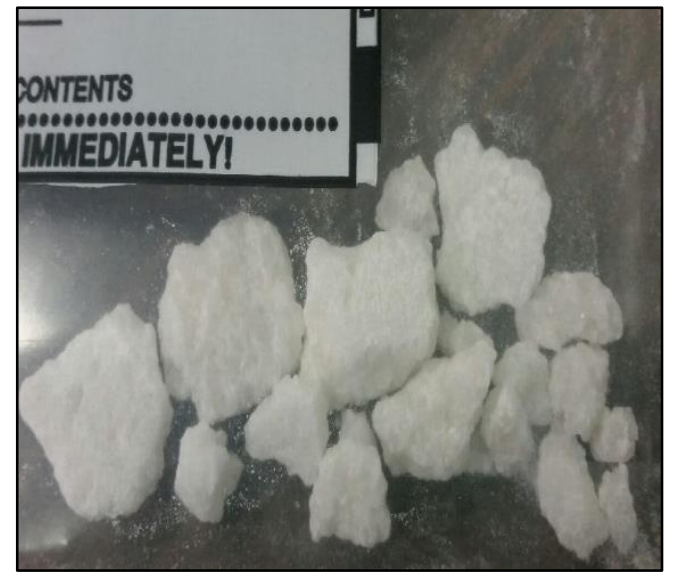

Figure 43. Visualization of Case 4
Case 259863: 11.4 grams of a substance was obtained from an uncover agent. The material was packaged in a clear Ziploc bag (dimension $3 \times 3$ in). The substance was bright white and compacted in a rock formation. Visual observation also revealed finite crystals jagged from the surface. The evidence container identified the substance as Ethylone.

Case 825156: 1,036 grams of a substance was confiscated in a large Ziploc bag stored inside of an aluminum bag. The bag was wrapped four times in a clear bubble wrap. The substance contained multiple brown rocks and a dense brown powder. The substance appeared to have a gloss and possessed a textile similar to wet sand. Presumptive testing suggested the

package contained 3,4-Methyethylone. 


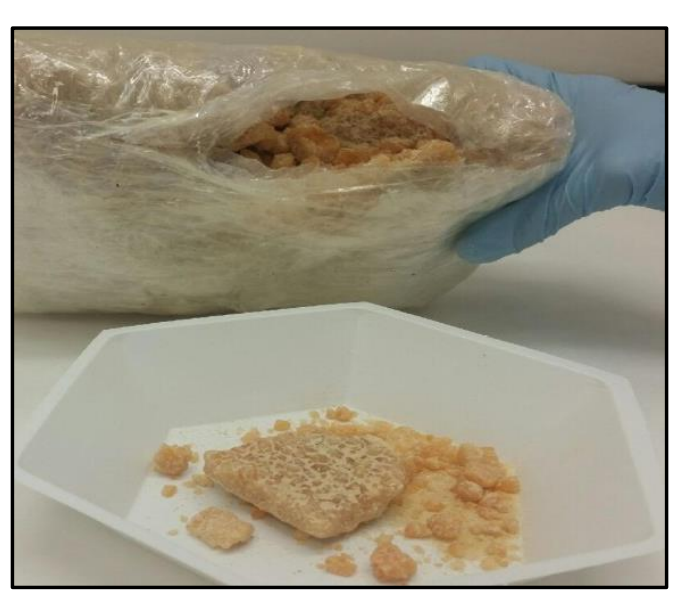

Figure 45. Visualization of Case 6

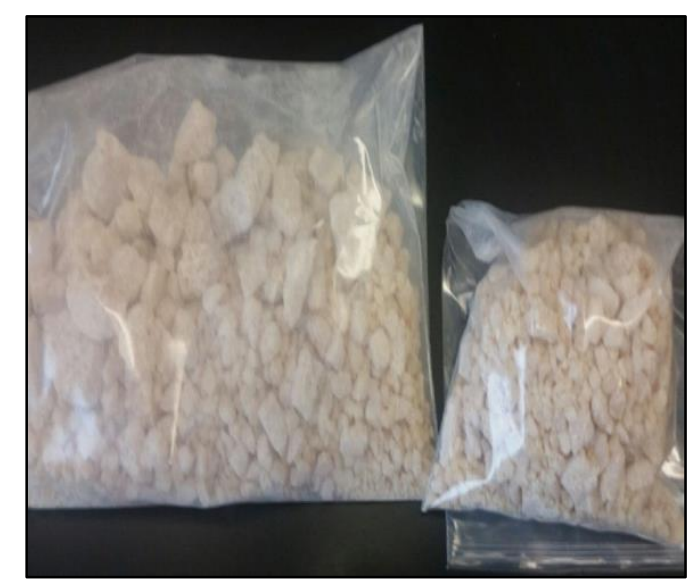

Figure 46. Visualization of Case 7
Case 194503: 1,513 grams of a substance was confiscated in a clear plastic bag and wrapped multiple times in clear saran wrap. The package contained a bright orange powder with very large rocks. The package appeared to be a large compressed brick. Presumptive testing suggested the package contained Ethylone.

Case 115160: Two separate, clear Ziploc bags were obtained. The larger bag contained 1,197 grams of a substance. The second bag contained 689 grams of the same substance. The substance in each container possessed an offwhite color. The material had a rock consistency of various sizes. Each rock appeared to be coated in a fine powder. Colorimetric presumptive testing suggested the substance was Ethylone. 


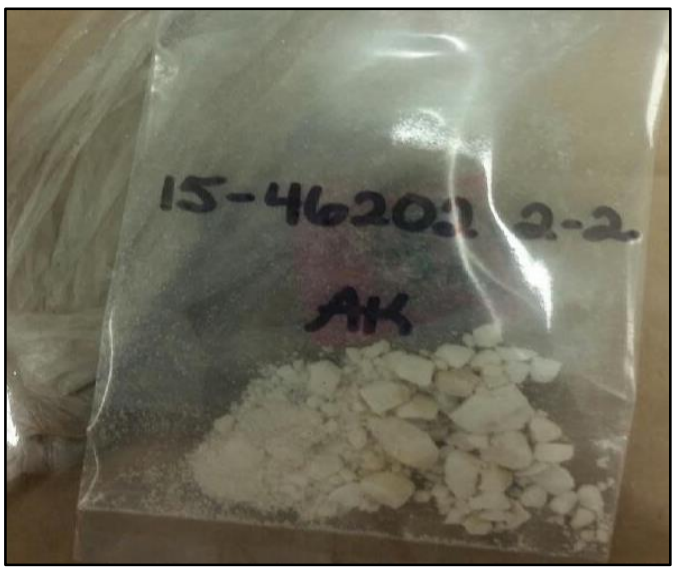

Figure 47. Visualization of Case 8
Case 1546202: A light brown plastic bag with a signal knot was confiscated. The substance within the bag consisted of very small pebbles and a powdered mixture. The rocks had a light brown and off-white color. The total weight of the sample was 1.079 grams. Colorimetric presumptive testing suggested the substance was $\alpha$-PVP.

\section{Case 1529523: A small re-sealable bag} containing 13.40 grams of a substance was confiscated. The substance had an off-white color. The package contained very small pebbles, with a large powder residue at the bottom of the bag. Colorimetric presumptive testing suggested that this bag contained

Figure 48. Visualization of Case 9 Ethylone. 


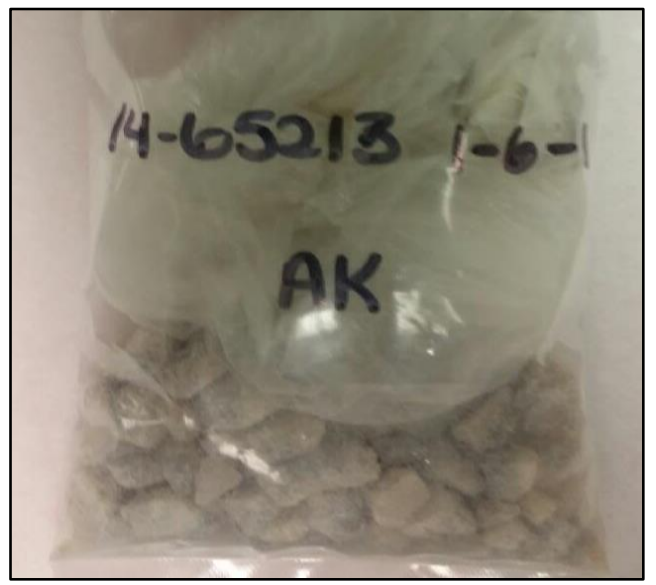

Figure 49. Visualization of Case 10

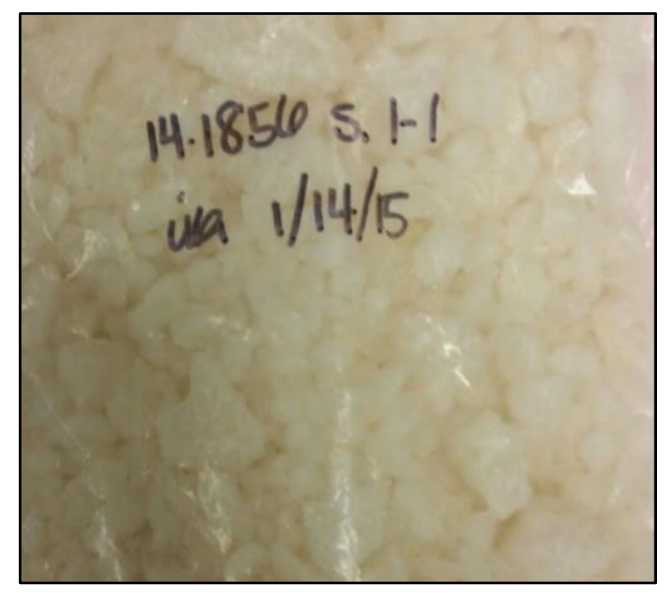

Figure 50. Visualization of Case 11
Case 1465213: A medium-sized semi-clear plastic bag containing 21 grams of a substance was confiscated. The substance contained medium to small rock formations. The base of the color was a brown shade with a mix of a darker brown tone. Colorimetric presumptive testing stated that the substance was Ethylone.

Case 141856: A large re-sealable bag containing 1,576 grams of an off-white substance was obtained. The package consisted of small white rocks. The rocks were lightly compressed and mild handling caused breakdown into a rough powder. Colorimetric presumptive testing suggested that this bag contained $\alpha$-PVP. 


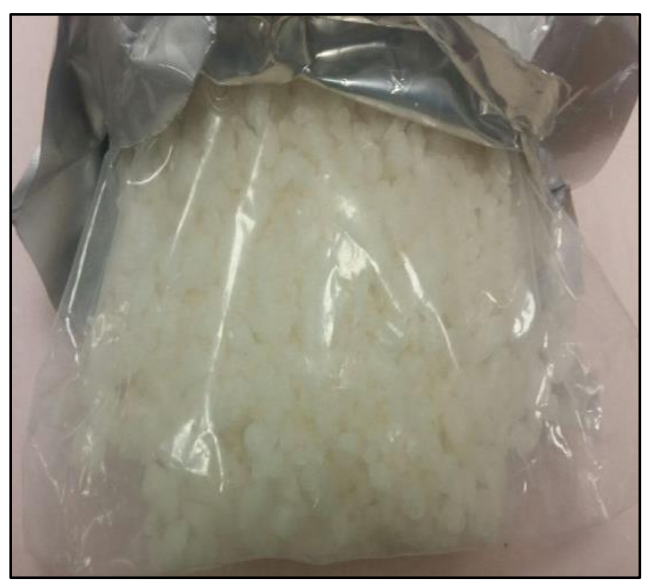

Figure 51. Visualization of Case 12

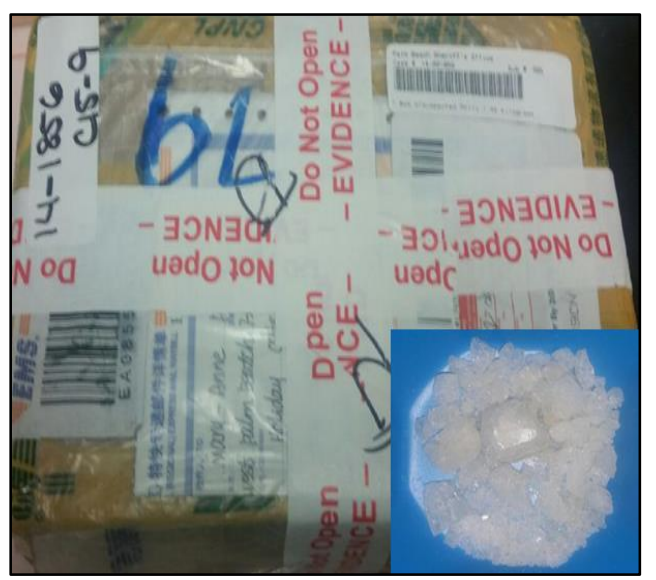

Figure 52. Visualization of Case 13
Case 141857: A large clear foldable bag was housed inside of an aluminum bag. The inner bag contained 1,134 grams of a rock like substance. The rock material was an off-white color. Colorimetric presumptive testing suggested that this bag contained $\alpha$-PVP.

Case 141859: A large brown shipping package from China was confiscated containing 1,700 grams of an unknown substance. Within the package there were compressed crystalline like material. The substance was a pure white color. Colorimetric presumptive testing suggested that this bag contained $\alpha$-PVP. 


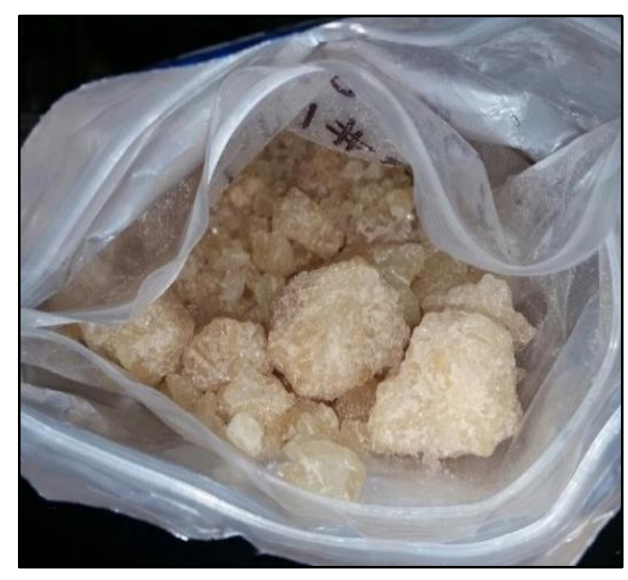

Figure 53. Visualization of Case 14 this bag contained $\alpha$-PVP.

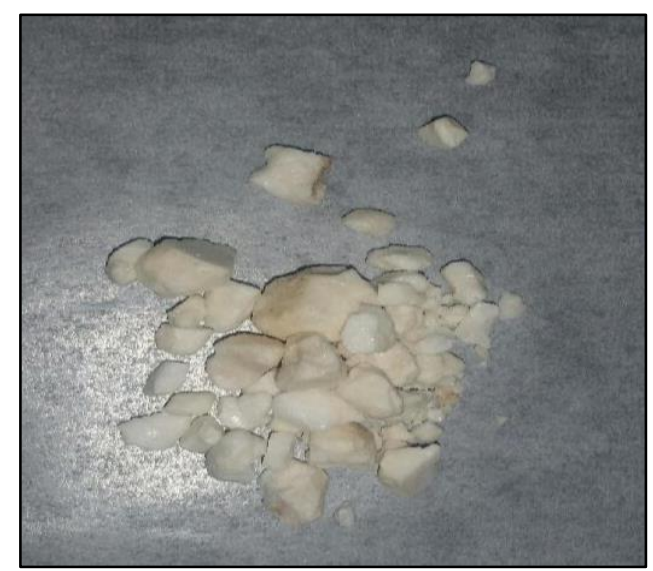

Figure 54. Visualization of Case 15
Case 14726: A large blue re-sealable shipping bag was confiscated. Inside this bag there was a clear re-sealable bag containing a rock-like substance. The overall product weighed 1,079 grams. The rock like substance possessed a mixture of off-white and light brown coloration. Colorimetric presumptive testing suggested that

Case 1546200: A small bag was confiscated containing 15 capsules and a small uncapsulated substance. The substance possessed very small rocks of a white and slightly off-white color. The total weight of the un-encapsulated substance was approximately 2.7 grams. Colorimetric presumptive testing suggested that this bag contained Ethylone. 


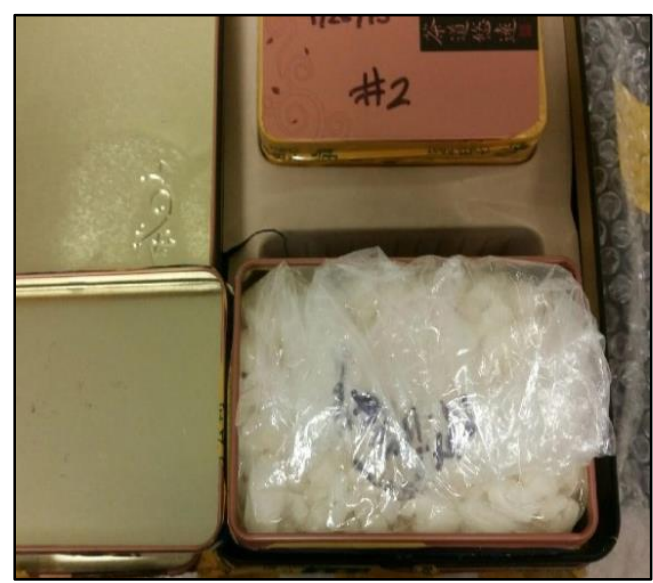

Figure 55. Visualization of Case 16
Case 141858: A large brown tea carrier was confiscated as a shipment from China. Opening the top lid revealed three smaller compartments. Removing the lids for each compartment revealed three clear bags folded into the canisters. Inside the bags there was an off-white medium rock-like substance. The weight of one bag was 596 grams. Colorimetric presumptive

testing suggested that the bags contained $\alpha$-PVP.

Applying the optimized extraction and separation technique to the sixteen confiscated drugs, we were able to develop the odor profile for each case. Displayed in figure 56, there was a vast amount similarities and distinguished differences between each case. Initial observations revealed that along with the chemical ionization method all parent drugs including methylone, ethylone, $\alpha-p v p$, and 3,4-methethylone were positively identified. Within the odor profiles analyzed any secondary cathinone derivative was also detected. For example case 14-726, identified as containing $\alpha$-pvp only within the crime lab, also contained ethylone within the headspace. 


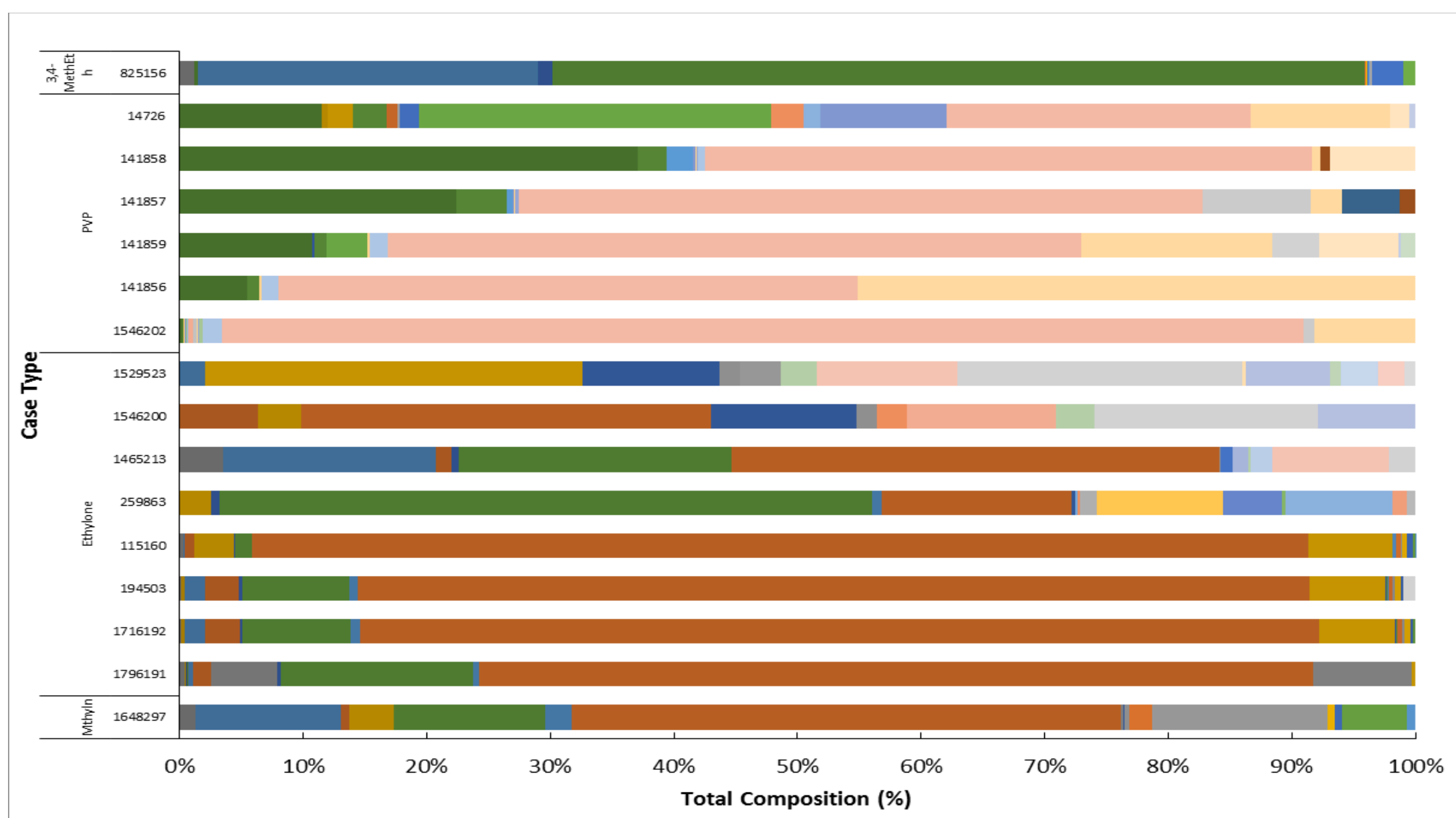

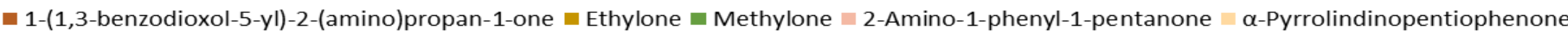

Figure 56. The Volatile Organic Compound (VOC) Profile of Sixteen Different Confiscated Synthetic Cathinones using the Optimal Headspace-Solid Phase Micro-Extraction (HS-SPME) Method 
In addition to the parent drugs identified within each case, the VOC profiles developed also highlighted any cutting agents or additives used within the product that was not detected by the instruments within the crime lab. Headspace extraction of the samples revealed triacetin, benzoin, butylated hydroxytoluene, or a variation of these compounds present in all sixteen samples analyzed. These compounds are typically used as an addictive to any narcotic to stretch the quantity of the drug. For example, if the drug only containing 100 grams, adding 50 grams of an addictive would lessen the power of the drug but provide more to sell.
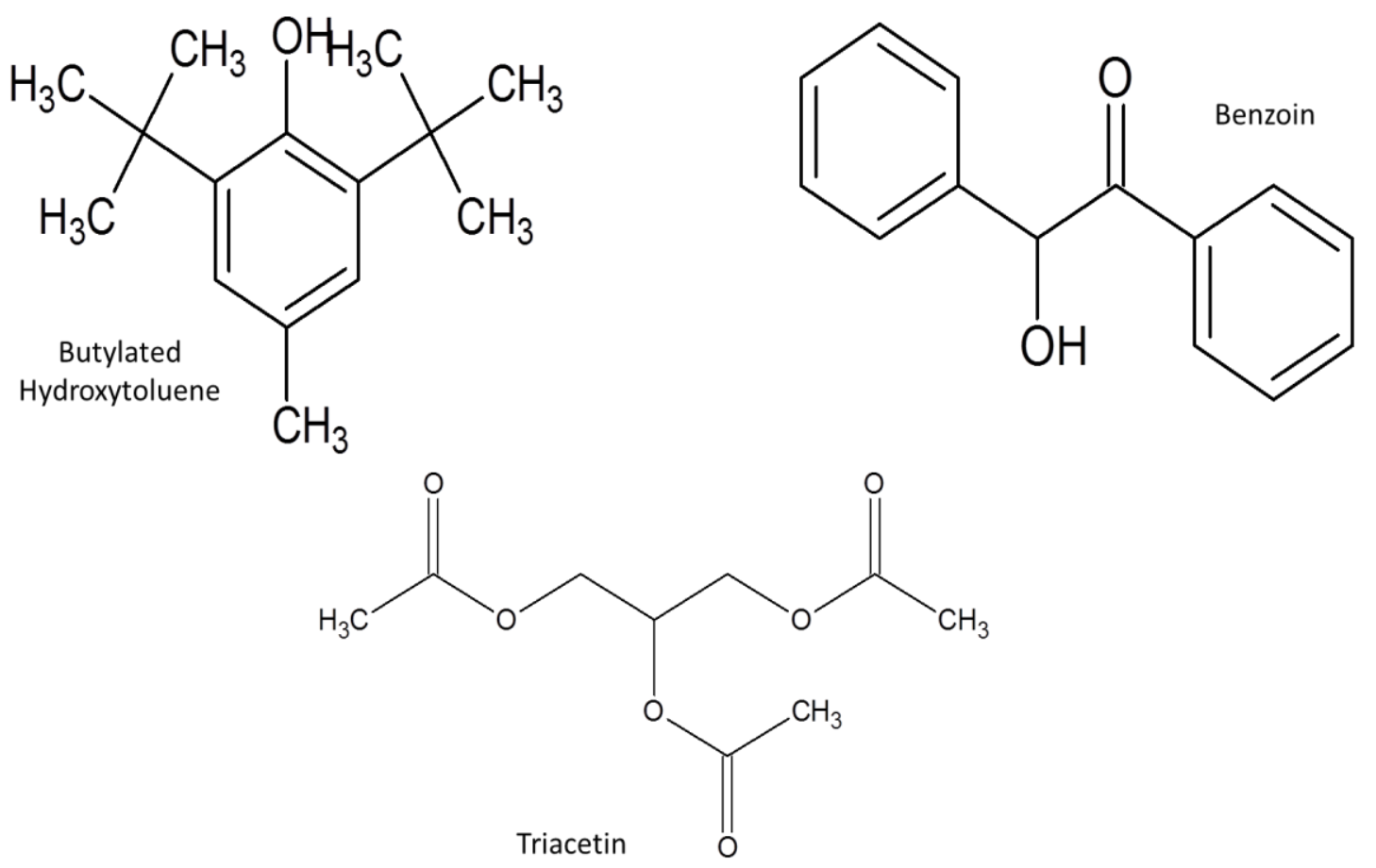

Figure 57. Cutting Agents Identified within the VOC Profiles of Analyzed Synthetic Cathinones 
A large portion of the odor profile developed was contributed to two deduced compounds: 2-Amino-1-phenyl-1pentanone and 1-(1,3-benzodioxol-5-yl)-2(amino)propanone. These two compounds were a direct breakdown from the parent drugs, where all the PVP cases produced the first compound and the remaining cases produced the later. The appearances of these compounds are explained by the continuous breakdown of the parent drug to produce these degraded products. In addition, the occurrence of these compounds is also a result of their formation as by-products identified during various synthetic routes uncovered.

The wide range of organic compounds identified when analyzing the profiles suggested the synthesized pathway taken to create the desired parent drug. These compounds showcased the likelihood of creating the targeted cathinone derivative in addition to creating other derivatives as a byproduct from undesired reactions given any experimental condition. Although crime labs are only able to detect the major product produced, the VOC profiles included all major and minor products, byproducts, starting material, and transitional products presumptively within the synthetic cathinone (table 24). The compounds identified in table 24 and figure 56, demonstrate the molecular information that can be missed during confirmative testing within a crime lab and the various characterizing factors that not only showcase the differences between the different synthetic cathinones; but also the various amount of similarities encountered. 
Table 24. The Organic Compounds Detected Within the Headspace of Various Synthetic Cathinone Samples

\begin{tabular}{|c|c|}
\hline Acid & $\begin{array}{l}\text { Number of Occurrences out } \\
\text { of } 16\end{array}$ \\
\hline Acetic acid & 8 \\
\hline dl-Aspartic acid & 3 \\
\hline Benzoylformic acid & 3 \\
\hline Alcohol & $\begin{array}{l}\text { Number of Occurrences out } \\
\text { of } 16\end{array}$ \\
\hline Tert-Butyl-phenol & 1 \\
\hline 2,4-Bis (1,1 dimethyl)-phenol, & 8 \\
\hline 2-Hexyl-1-octanol & 2 \\
\hline Butylated Hydroxytoluene & 6 \\
\hline 3-Ethyl-7-hydroxyphthalide & 2 \\
\hline Ethyl mandelate & 10 \\
\hline Triacetin & 5 \\
\hline Aldehyde & $\begin{array}{l}\text { Number of Occurrences out } \\
\text { of } 16\end{array}$ \\
\hline 3,5-Dibromosalicaldehyde & 7 \\
\hline 3,5-Dibromo-4-hydroxybenzaldehyde & 1 \\
\hline 3-Bromo-5-chloro-2-hydroxybenzaldehyde & 3 \\
\hline Piperonal & 7 \\
\hline Amine/Amide & $\begin{array}{l}\text { Number of Occurrences out } \\
\text { of } 16\end{array}$ \\
\hline 2,3-Dimethoxy-5-aminocinnamonitrile & 1 \\
\hline Acetamide, N-cyclohexyl- & 2 \\
\hline Pyrrolidine, 1-(1-oxobutyl)- & 5 \\
\hline Pyrrolidine, 1 -acetyl- & 6 \\
\hline 1,2-Ethanediamine, N,N'-dibutyl- & 1 \\
\hline Esters & $\begin{array}{l}\text { Number of Occurrences out } \\
\text { of } 16\end{array}$ \\
\hline Ethyl ester Benzoic acid & 5 \\
\hline 4-cholorphenyl benzoate & 2 \\
\hline Diethyl phthalate & 7 \\
\hline Hexanedoic acid, bis (1-methylethyl) ester & 4 \\
\hline $\begin{array}{l}\text { 1,3-Benzodioxole-5-carboxylic acid, } \\
\text { methyl ester }\end{array}$ & 3 \\
\hline Fenchyl acetate & 2 \\
\hline $\begin{array}{c}\text { 2-Propenic acid, 3-(4-fluorophenyl)-,ethyl } \\
\text { ester }\end{array}$ & 1 \\
\hline Butanoic acid, bis (1-methylethyl) ester & 1 \\
\hline
\end{tabular}




\begin{tabular}{|c|c|}
\hline pentanedioic acid, dimethyl ester & 1 \\
\hline Halogenated Compounds & $\begin{array}{l}\text { Number of Occurrences out } \\
\text { of } 16\end{array}$ \\
\hline 4-Bromo-1,2-(methylene) benzene & 9 \\
\hline 5-Chloro-1,3-benzodioxole & 2 \\
\hline 1,1-Dichloro-2,2-diethoxyethane & 2 \\
\hline Broxaldine & 2 \\
\hline 2-Bromo-1-(4-methylphenyl)-ethanone, & 3 \\
\hline Hydrocarbons & $\begin{array}{l}\text { Number of Occurrences out } \\
\text { of } 16\end{array}$ \\
\hline Diphenylmethane & 2 \\
\hline Naphthalene & 1 \\
\hline Pentamethylbenzene & 1 \\
\hline 1,3-Benzodioxole & 10 \\
\hline 2-Methylnaphthalene & 4 \\
\hline Ketone & $\begin{array}{l}\text { Number of Occurrences out } \\
\text { of } 16\end{array}$ \\
\hline 1-Phenyl-1-pentanone, & 10 \\
\hline 3',4'-Methylenedioxyacetophenone & 7 \\
\hline 3,4-Methylenedioxypriopiophenone & 12 \\
\hline $\begin{array}{l}\text { 1-(1,3-benzodioxol-5-yl)-2-(amino)propan- } \\
1 \text {-one }\end{array}$ & 8 \\
\hline 1-Phenyl-1-butanone & 1 \\
\hline Cyclobutyl phenyl ketone & 3 \\
\hline 2-Amino-1-phenyl-1-pentanone & 6 \\
\hline Methylone & 1 \\
\hline 2-Chloro-Acetophenone & 2 \\
\hline Ethylone & 6 \\
\hline 3,4-Methylenedioxyphenyl acetone & 2 \\
\hline$\alpha$-Pyrrolindinopentiophenone & 6 \\
\hline 2-Bromoisobutyrophenone & 1 \\
\hline Benzoin & 3 \\
\hline
\end{tabular}

Overall, by analyzing the synthetic cathinones submitted to various crime labs, the optimized extraction method created for the detection of the various odor profiles was successful. Applying a PDMS fiber, along with the extraction parameters and chemical ionization proved to be adequate selections required to detect a wide range of compounds 
contributing to the odor profile of these synthetic drugs. Within this task the odor profiles developed identified the gap of knowledge that is continuously missed when crime labs perform analytical testing of synthetic cathinones and potentially other drugs routinely encountered. This task demonstrated the power of HS-SPME to unveil the vast amount of cutting agents that are often added to drugs in addition to the impurity and purity of any given synthetic cathinone. A large conclusion drawn from this task was that although synthetic cathinones do vary in the parent drug contained and physical appearance, the odor profiles overall do bare a vast number of reoccurring compounds.

\subsection{Imprinting Canine to Detect Synthetic Cathinones}

\subsubsection{Detection of Synthetic Cathinones via Currently Deployed Narcotic Detection Canine Teams}

All canines tested were previously trained to detect a wide range of routinely encountered drugs including cocaine, marijuana, heroin, methamphetamine, and MDMA. While synthetic cathinones are structurally similar to both MDMA and methamphetamine, it was initially theorized, by law officials, that the odor would also be similar amongst these drugs. Those similarities would support the idea that currently imprinted canines for the detection of MDMA and methamphetamine could successfully detect synthetic cathinones. As development of the odor profiles were established and compared to the profile of both MDMA and methamphetamine (table 10 and 11). Theoretically, canines currently deployed and trained to detect both MDMA and meth, could not detect synthetic cathinones samples, although the drugs were structurally similar. 
The later theory was tested to determine the detection capability of currently deployed canines. Initial testing began in Miami-Dade County with the Miami Dade Police canine team. The cases as described in section 4.4 were placed more than 5 feet apart and allowed time to develop an odor plum. Each canine team was ran on the cases and the results are highlighted in table 25 .

Table 25. Detection Capabilities of Narcotic Detection Teams Deployed in Miami-Dade County

\begin{tabular}{|c|c|c|c|c|c|}
\hline Canine No. & PC 1 & PC 2 & Methylone & Ethylone & Blank \\
\hline 13 & $3 / 3$ & $3 / 3$ & $0 / 3$ & $0 / 3$ & $0 / 3$ \\
\hline 65 & $3 / 3$ & $2 / 3$ & $0 / 3$ & $0 / 3$ & $0 / 3$ \\
\hline 76 & $3 / 3$ & $3 / 3$ & $0 / 3$ & $0 / 3$ & $0 / 3$ \\
\hline 87 & $3 / 3$ & $3 / 3$ & $0 / 3$ & $0 / 3$ & $0 / 3$ \\
\hline 89 & $3 / 3$ & $3 / 3$ & $0 / 3$ & $0 / 3$ & $0 / 3$ \\
\hline Alert Response & $100 \%$ & $94 \%$ & $0 \%$ & $0 \%$ & $0 \%$ \\
\hline
\end{tabular}

As depicted, in table 25, all five of the canines were able to accurately detect the presence of both the marijuana and MDMA (positive control). The working capability of each dog was validated to assure they were able to identify previously trained narcotics. Exposure of the canines to each case showed that none were able to detect either synthetic cathinone. For the methylone case, none of the dogs alerted. The odor produced from ethylone did not facilitate any alerts from the canines. Some of the working dogs did express an interest in the ethylone odor but were not able to connect with an olfactory receptor as a learned odor. 
The second canine trial conducted was with the Palm Beach County canine teams under the same guidelines. Based on recommendations of the trainer, the teams were most optimal with detection involving lockers. The preliminary trial was performed at the Palm Beach High School to ensure that the most efficient biological detection was being used for the experiment. The cases involved were 17-426, 14-1856, 15-02913, and 1465213; the first two containing $\alpha$-PVP and the later Ethylone. These cases were selected for testing because they presented a wide range of versatility in the odor profiles developed for each case (refer to figure 56). Although cases were placed inside locker seams the distance recommended by SWGDOG guidelines were still implemented; one case per locker corridor. In addition, heroin and methamphetamine were used as positive controls during testing. A large denomination of shredded money (approximately $\$ 1,000$ ) was used as the blank during the trial. A total of seven canine teams were run on the markers and observations were configured in table 26.

Table 26. Detection Capabilities of Narcotic Detection Teams Deployed in Palm Beach County

\begin{tabular}{|c|c|c|c|c|c|c|c|}
$\begin{array}{c}\text { Canine } \\
\text { No. }\end{array}$ & PC 1 & PC 2 & $\begin{array}{c}\text { PVP } \\
\mathbf{1 7 - 4 2 6}\end{array}$ & $\begin{array}{c}\text { PVP } \\
\mathbf{1 4 - 1 8 5 6}\end{array}$ & $\begin{array}{c}\text { Ethylone } \\
\mathbf{1 5 - 0 2 9 1 3}\end{array}$ & $\begin{array}{c}\text { Ethylone } \\
\mathbf{1 4 - 6 5 2 1 3}\end{array}$ & Blank \\
\hline 1 & $2 / 2$ & $2 / 2$ & $0 / 2$ & $0 / 2$ & $0 / 2$ & $0 / 2$ & $1 / 2$ \\
\hline 2 & $2 / 2$ & $2 / 2$ & $0 / 2$ & $0 / 2$ & $0 / 2$ & $0 / 2$ & $0 / 2$ \\
\hline 3 & $2 / 2$ & $2 / 2$ & $0 / 2$ & $0 / 2$ & $2 / 2$ & $0 / 2$ & $0 / 2$ \\
\hline 4 & $2 / 2$ & $2 / 2$ & $0 / 2$ & $1 / 2$ & $0 / 2$ & $0 / 2$ & $0 / 2$ \\
\hline 5 & $2 / 2$ & $2 / 2$ & $1 / 2$ & $0 / 2$ & $0 / 2$ & $1 / 2$ & $0 / 2$ \\
\hline 6 & $2 / 2$ & $2 / 2$ & $0 / 2$ & $0 / 2$ & $2 / 2$ & $0 / 2$ & $0 / 2$ \\
\hline 7 & $2 / 2$ & $2 / 2$ & $0 / 2$ & $0 / 2$ & $0 / 2$ & $0 / 2$ & $0 / 2$ \\
\hline $\begin{array}{c}\text { Alert } \\
\text { Response }\end{array}$ & $100 \%$ & $100 \%$ & $7.1 \%$ & $7.1 \%$ & $28.5 \%$ & $7.1 \%$ & $7.1 \%$ \\
\hline
\end{tabular}


With the large facility used and number of canines present for this trial, each canine was run twice through the set up to reconfirm initial alerts. As suspected, we were able to confirm that the Palm Beach Detection teams were operating properly; as all the canines were able to positively identify the narcotics previously imprinted on (the positive predictive value was $100 \%$ ). From the trial conducted we were able to make several observations. The largest conclusion drawn from this trial was that canines could not actively alert to the synthetic cathinone cases. For cases $17-426,14-856,14-6513$ the alert percentage was approximately $7 \%$ (negative predictive value was $95 \%$ ). This alert percentage was calculated as a result of the canines showing interest to each bath salt, when exposed to the drug within the first or second run. The interest was marked as the canines expressed a prolonged sniffing motion at one locker before moving on to the next corridor. Although there was interest shown for the three cases, no canine physically signaled a final alert. The confiscated currency used as a blank during this trial elicited some interest by one canine during the first run but no response upon the second exposure. The Ethylone case (15-02913) had an overall alert rate of approximately 28\% with a low PPV of $27 \%$. During both runs there were two working dogs that alerted to the sample. Although, these searches were denoted as alerts, none of the canine actively showed any indication of an alert. The alerts were suggested observations dependent on the handler. Each handler was given the chance to call out a resulting response based on their canine's behavior. 
Overall, from the preliminary trials performed both in Miami-Dade and Palm Beach counties, it was determined that currently deployed canines teams were not equipped with the necessary tools required for the detection of a synthetic cathinone sample. As supported by the odor profiles developed, although the parent drug of MDMA and various cathinone derivatives may be similar in their chemical structure; these drugs did not facilitate the same response from any canine team in comparison to MDMA and methamphetamine. In order to address the detection limitations of bath salts, it was necessary to use these canines as the tool of identification and detection.

\subsubsection{Novel Odor Introduction}

There were three phases used to imprint the canines on the synthetic drugs. The teams were divided into two, where half were imprinted on the $\alpha$-pvp cases and the other half on the ethylone cases. This division was imposed to test the ability of each canine to accurately detect the presence of other cathinone derivatives not imprinted on; therefore supporting the concept that each type of cathinone derivative contained the same odorant responsible for each alert. The first phase of imprinting was introducing the canine to the odor using popper boxes. The purpose of this phase was to allow the dogs to become familiar with the odor. By bringing the dog to the box containing the odor and allowing the canine to engage for an extended period of time; the handler began the recognition process between the odor and the canine's olfaction. Once the towel was released as a positive reward, the dog began to develop a connection between the new odor and positive payment. Each canine team was able to successfully pass this stage of the imprinting process within two weeks. 
Phase two of imprinting was introducing the concept of seeking and finding the odor in order to receive the positive reward. This phase required the canine to not only engage in the sniffing motion but to actively search the odor to obtain the reward. The canines were successful in this phase as well. Three working dogs required an extended period within the second phase. These canines were older and progressed at a slower rate in comparison to the younger detection teams. After the canines were taught to recognize, find, and translate a response to their respective handlers of the odors present; the last phase was testing out how efficient the canine was at detecting the odor in the presence of other distracting odors.

The first distractor tested was tennis balls. These balls were typically used as toys for the dogs and were placed to test the canine's selectivity. Majority of the canines were able to avoid the odors from the tennis balls and focus on seeking each bath salt case, however, one canine struggled with this task. When lack of avoidance and obedience took place (i.e., the canine would not actively search or would continuously engage the distractors), the canine was brought to the start of the lineup and instructed to repeat the active search again until the sample was identified. Before rewarding (paying) the dog, the handler would walk away from the canine or create a pause to build confidence in the canines' sense of detection and olfactory capabilities. Afterwards, the lineups included the use of dog food. This distractor was used to test the canine's drive and determination to work (i.e., conduct a search) over their animal instinct to consume. Initially four of the canines were not able to pull from the dog food included. Through various sessions and multiple corrections the lineup and runs were repeated until the canines were able to detect the odor to obtain an award. 


\subsubsection{Imprinting Validation Study}

After the canines were imprinted on the samples, the next phase was validating that the canines successfully moved through the training process and were imprinted on synthetic cathinones. A canine trial was conducted to test whether the canines could identify and detect bath salts, in addition to determining if the canines independently imprinted on one variation of the drug could detect the other. The canine trial was conducted at the Palm Beach Canine Training Facility. The lineup used during the canine trial included boxes similar to those used during the imprinting stage and were stored in various locations within the building. The results observed during this validation trial are highlighted in table 27.

Table 27. Validation Test for Newly Imprinted Canines on Synthetic Cathinones

\begin{tabular}{|c|c|c|}
\hline \multicolumn{3}{|c|}{ C(n=12) } \\
\hline Component & $\begin{array}{c}\text { Group A Combined Alert } \\
\text { Rate (\%) }\end{array}$ & $\begin{array}{c}\text { Group B Combined Alert } \\
\text { Rate (\%) }\end{array}$ \\
\hline Blank & 0.0 & 0.0 \\
\hline Marijuana & 100.0 & 100.0 \\
\hline MDMA & 100.0 & 100.0 \\
\hline Case 14-856 & 100.0 & 100.0 \\
\hline Case 16-065213 & 100.0 & 100.0 \\
\hline Case 15-02953 & 100.0 & 100.0 \\
\hline Case 14-726 & 100.0 & 100.0 \\
\hline
\end{tabular}


After a month of undergoing imprinting and training for the detection of synthetic cathinones, the validation trial concluded that all canines had been successfully imprinted on the odor of these drugs. The canines imprinted on $\alpha$-PVP (group A) were able to detect the Ethylone cases and the same phenomenon was witnessed with group B (imprinted on the Ethylone cases).

Initial examination of the canines within task 4 resulted in two conclusions. The first conclusion was that the canines teams currently deployed in South Florida were not able to detect synthetic cathinones; given what they were already imprinted on. Although the canines were sufficient at detecting more traditional drugs including heroin, MDMA, methamphetamine, and cocaine; the odors used to detect those drugs did not facilitate the same alert when the canines were exposed to the synthetic cathinones. The second conclusion drawn was that canines could be imprinted on the cathinones derivatives to learn the odor and detect it. As shown above, through training and behavioral assessment, the canines were able to learn a new odor associated with synthetic cathinones and detect it within a given parameter. Once the canines were able to detect the cathinone derivatives, the odorant responsible for their alert could be determined.

\subsection{VOC Isolation for Development of a Controlled Odor Mimic Permeation System}

\subsubsection{Development of Organic Compound Delivery System}

As stated in section 4.5, the delivery of the compounds to the canine's nose was conducted by employing a gauze stored inside of a permeable bag. There were two categories for compound introduction: direct application of a standard or a high 
concentrated diluted sample. For the standards purchased in the solid form it was ideal to dilute the sample into a liquid state prior to introduction to the gauze; thus promoting a stronger interaction between the absorbent and compound. All compounds were determined to be soluble in methylene chloride. In order to ensure the sole delivery of the selected compound, the complete dissipation of the solvent had to be accomplished.

To successfully transfer 10mg of any given compound, $100 \mu \mathrm{L}$ of a $100,000 \mathrm{ppm}$ solution was pipetted onto the surgical gauze. To determine the time required for the complete dissipation of the solvent, a delivery bag was created without the presence of the odorant. A permeable bag storing one gauze pad (pre-weighed) was spiked with $100 \mu \mathrm{L}$ of pure methylene chloride and tripled heat sealed. Gravimetrical evaluation was performed over time until the delivery bag returned to its original weight. Triplicates of the evaluations were performed and plotted to determine the length of time (figure 58). Based on the analysis, it was determined that the length of time required for the complete dissipation of methylene chloride was 45 minutes. Once commenced, the solvent would be completely evaporated leaving behind $10 \mathrm{mg}$ of the selected odorant bound in the weaving of the gauze pad. 


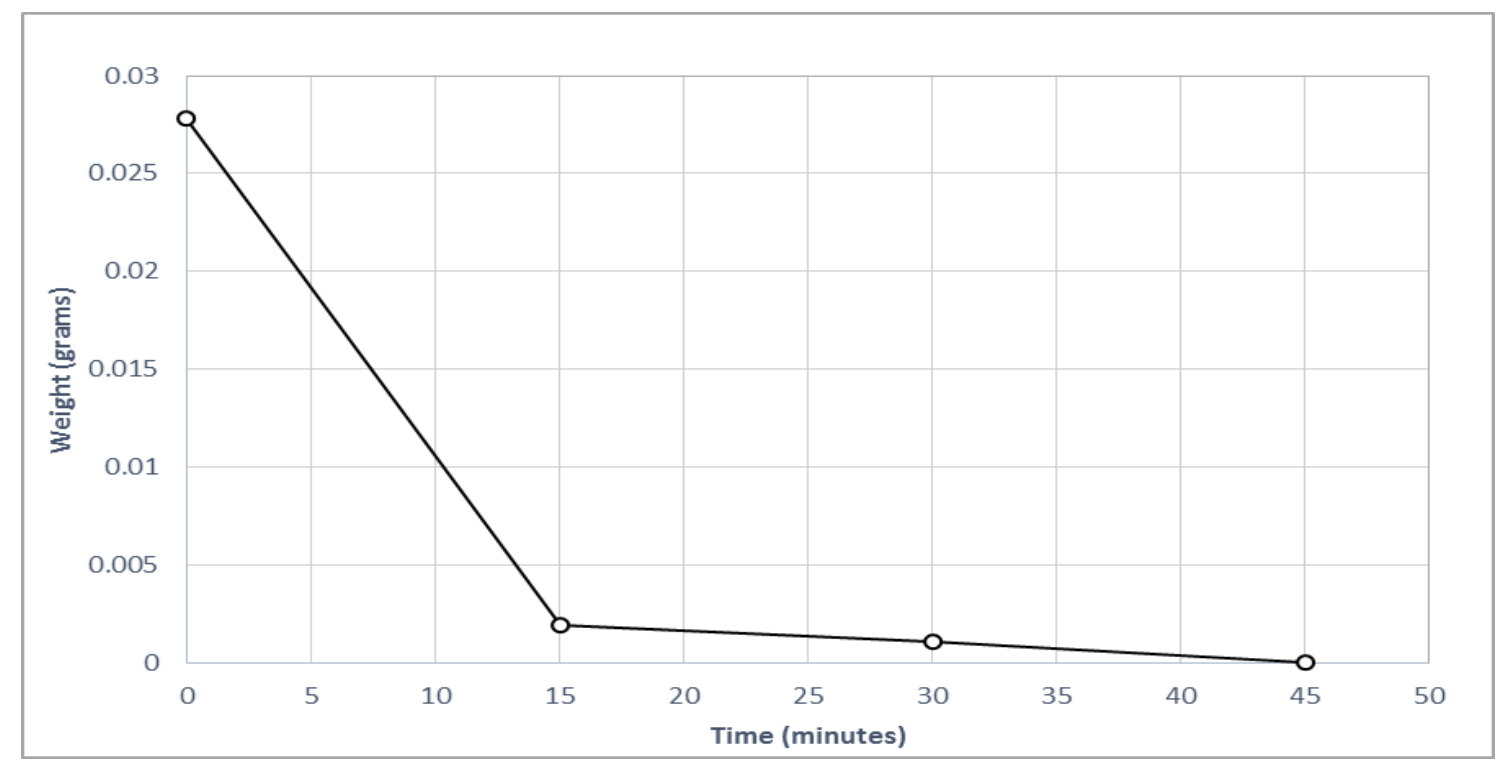

Figure 58. The Allotted Time Required for the Complete Dissipation of Methylene Chloride from a Surgical Gauze Pad

5.5.2 Compound Introduction to Canines for the Determination of the Active Odorant

The lists of odorants identified from the odor profile to be initially exposed to the canines are listed in table 28. These compounds were selected based on the fact that they were present in more than one case and more than one type of cathinone derivative analyzed. In addition, the compounds selected also contributed to some of the largest abundances detected in the odor profiles. The objective was to determine a universal active odor that could be used to detect a wide range of synthetic cathinones once imprinted and trained. 
Table 28. Compound Exposure List for Active Odor Determination

\begin{tabular}{|c|c|}
\hline Compound & CAS Number \\
\hline Valerophenone & $1009-14-9$ \\
\hline Ethyl Benzoate & $93-89-0$ \\
\hline 3,4-Methylenedioxypropiophenone & CDS001550 \\
\hline 1,3-Benzodioxole & $274-09-9$ \\
\hline $\boldsymbol{\alpha}$-PVP & P-090 \\
\hline Ethylone & E-071 \\
\hline Isobutyrophenone & $611-70-1$ \\
\hline 2-Acetoxphenone & $451-40-1$ \\
\hline Ethyl Mandelate & $774-40-3$ \\
\hline Naphthalene & $91-20-3$ \\
\hline
\end{tabular}

Both standards of the parent drug were included in the lineup of odors. The reason behind this was to assess whether or not the parent compound, which caused the euphoric effect, was also responsible for the positive canine response. In addition, the positive controls per trial consisted of the actual drugs, MDMA, and marijuana. All odor deliver COMPS were created the night before, stored in a pelican case, and kept at cool temperatures (approximately $12^{\circ} \mathrm{C}$ ) until the trail was conducted.

Periodically the canine trials were relocated to accommodate the weekly training sessions of the teams. The first canine trial conducted was on the Palm Beach County Canine Training Facility site. Each COMPS was taken on out of its perspective vial and stored inside a sterilized mason jar attached to a BSD K-9 popper box. Each box was stored in a separate room. The boxes containing the standards were placed in a separate 
outdoor corridor. The bags were allowed 30 minutes to accumulate a detectable odor. Each canine was run on the odors twice and the elicited responses were noted.

The second canine trial was conducted at the Palm Beach Airport off-site training facility. An abandon building with four large servicing rooms (37 x 98 sq. feet each) were used for the trial. Within each room there were 2 cinderblocks stacked and separated by more than five feet. Each room contained sixteen cement blocks and a row of boxes. Each COMPS was taken out and placed in the upper section of the cement block. Each COMPS bag was separated by a cement block to prevent cross contamination. Once again the canines were allowed to engage the lineup twice while the observations were noted.

The third trial was performed at the Palm Beach School initially used during the preliminary trials. During this trial, the setup was located in a hallway. Trails of lockers were cleared out on the first level of the school and a single COMPS bag was placed at various locations within each corridor. The first synthetic cathinone case (17-426) was placed at the start of the hallway, cases $14-65213$ and 14-1856 were placed in the middle, and case 15-02913was located at the end of the search. The positions of the cases were used to motivate and encourage the canines to continue conducting the search. As previously stated the study was conducted by allowing the headspace to accumulate while handlers were instructed of the same procedures.

From the canine trials and replications, the responses were recorded in table 29. As expected based on prior imprinting and training, all canines were able to accurately detect all the bath salt cases in addition to the previously imprinted drugs (marijuana and 
MDMA) included in the lineup. After the initial trials, 2-acetoxphenone, naphthalene, and ethyl mandelate were eliminated from consideration based on the fact that none of the canines showed interest or alerts to this odor (NPV was 100\%). Isobutyrophenone and valerophenone were also eliminated from consideration. With a small alert potential of only $14.3 \%$ and approximately $15 \%$ between the two compounds there was no definitive indication of recognition or interest in the odors present for continuation. 1,3benzodioxole was eliminated after it showed a lower alert rate of only $20 \%$ for group A (imprinted on PVP) and none for the second group. Although the canines did show interest for an extended period of time with this odor, 1,3-benzodioxole could not be used for detection of cathinone variations. 
Table 29. Canine Alert Response Tabulated from Various Odor Delivery Bags

\begin{tabular}{|c|c|c|}
\hline \multicolumn{3}{|c|}{$C(n=12)$} \\
\hline Component & $\begin{array}{c}\text { Group A Combined Alert } \\
\text { Rate }(\%)\end{array}$ & $\begin{array}{c}\text { Group B Combined } \\
\text { Alert Rate }(\%)\end{array}$ \\
\hline Blank & 0.0 & 0.0 \\
\hline Marijuana & 100.0 & 100.0 \\
\hline MDMA & 100.0 & 100.0 \\
\hline Isobutyrophenone & 14.3 & 0.0 \\
\hline Valerophenone & 14.3 & 16.7 \\
\hline 1,3-Benzodioxole & 20.0 & 0.0 \\
\hline Naphthalene & 0.0 & 0.0 \\
\hline Ethylone standard & 0.0 & 5.6 \\
\hline PVP standard & 20.0 & 0.0 \\
\hline Ethyl Benzoate & 90.0 & 40.0 \\
\hline 3,4-Methylenedioxypriophenone & 80.0 & 93.3 \\
\hline Ethyl Mandelate & 0.0 & 0.0 \\
\hline 2-Acetoxphenone & 0.0 & 0.0 \\
\hline Ethylone Case & 100.0 & 100.0 \\
\hline PVP Case & 100.0 & 100.0 \\
\hline
\end{tabular}

From the canine studies we were able to eliminate both the PVP and Ethylone standard. After careful notation of the dog's performance it was concluded that only $20 \%$ of the canines from group A were able to alert to the PVP standard, while all dogs were unsuccessful at identifying the Ethylone standard. Both the PPV and NPV for the PVP standard were calculated to be $8.3 \%$ and $92 \%$ respectively. The canines showed even less interest in the Ethylone standard they were introduced to. These findings support the fact 
that although the parent drug causes the psychoactive effort, the same compounds were not responsible for the canine's alert (positive response). In addition these findings also supported the creation of a non-controlled mimic training aid, making it safe to transport without confinement to strict regulations. For the canine trials performed there were two compounds which resulted in a relative high yield of canine alerts. The COMPS containing Ethyl benzoate was able to produce a $90 \%$ alert from the canines in group A while obtaining a $40 \%$ alert from the canines in group B. The largest observed alert was seen with the COMPS containing 3,4-Methylenedioxypropiophonene. Both groups achieved the highest alert rate with this compound ( $80 \%$ for group A and $93 \%$ for group B). With such a large response from the canines, binary mixtures of these two compounds were formed to determine if the alert rates would increase beyond what was achieved in singularity.

A canine trial was performed to determine the response to a 1:1 mixture (and variations) of both 3,4-methylenedioxypropiophenone and ethyl benzoate. The canine trials were conducted at the school and occupied two classrooms and a hallway. In the first classroom there were 5 cinderblocks containing a mixture of all the compounds initially tested and an imprinted case. In the hallway each corridor contained a single COMPS bag housing the binary mixtures, both odorants in separate bags, and the remaining imprinted cases. The second classroom contained the positive controls and was used as an active training site. Each handler was given the same instructions (i.e., that the trial was not a graded test and to vocalize any change in behavior experienced by the dog) and ran in the same sequence and manner as the previous trials. Canine responses were recorded in table 30 . 
Table 30. Canine Alert Response Tabulated from the COMPS Bags Containing Binary Mixtures

\begin{tabular}{|c|c|}
\hline \multicolumn{2}{|c|}{ C(n=6) } \\
\hline Hide Component & $\begin{array}{c}\text { Combined } \\
\text { Alert Rate (\%) }\end{array}$ \\
\hline Blank & 0.0 \\
\hline Ethylone Case & 100.0 \\
\hline$\alpha$ PVP Case & 100.0 \\
\hline $1: 1$ mix (3,4Meth: EthB) & 50.0 \\
\hline $2: 1$ mix (3,4Meth: EthB) & 60.7 \\
\hline $1: 2$ mix (EthB: 3,4Meth) & 16.7 \\
\hline
\end{tabular}

Canine trials performed with the binary mixtures revealed that no canine alerts were indicated higher than approximately $60 \%$ or $50 \%$, when the mixture contained an equal amount of the odorants or a larger quantity of the 3,4-

methylenedioxypropiophenone. Although $50 \%$ of the canines showed interest or were able to alert to the binary mixtures, majority of the canines positively identified $3,4-$ methylenedioxypropiophenone as the active odorant facilitating an alert in more than $87 \%$ of the canines imprinted on both PVP and Ethylone (when the canines were exposed to the odorant during the same trial).

Through various trials performed within this task, it was concluded that 3,4methylenedioxypropiophenone would be optimally selected to develop the pseudo training aid in the form of a Controlled Odor Mimic Permeation System (COMPS). From the odor profiles developed in task 4, reoccurring compounds initially were selected for canine introduction. Unlike the other odorants encountered, this compound continuously 
resulted in an alert rate higher than $85 \%$ for both canine groups. In addition, when the compound was included in any binary mixture, the canine's response displayed a proportional relationship with the odorant's quantity present. By isolating the compound tested within the task, the study was able proceed with development.

\subsection{COMPS Optimization for Synthetic Cathinones}

\subsubsection{COMPS: SPME Optimization}

Prior to the development of any COMPS the parameters used for extraction were reexamined. It was determined that 15 minutes equilibrium and 4 hours of SPME fiber exposure were sufficient parameters for the complete extraction and development of a complete odor profile for the synthetic cathinones. The parameters were reexamined to determine if the length of time could be lessen and still remain optimal for the extraction of a singular compound; 3,4-methylenedioxypropiophenone. Using 10-mL vials, 1x1 inch surgical gauze pads were placed in the bottom of each vial. Approximately $100 \mathrm{ng}$ of the odorant was deposited on the pad and the equilibration times were tested in triplicates. Initially the equilibrium was reassessed testing the times of $5,15,30$, and 60 minutes (figure 59). 


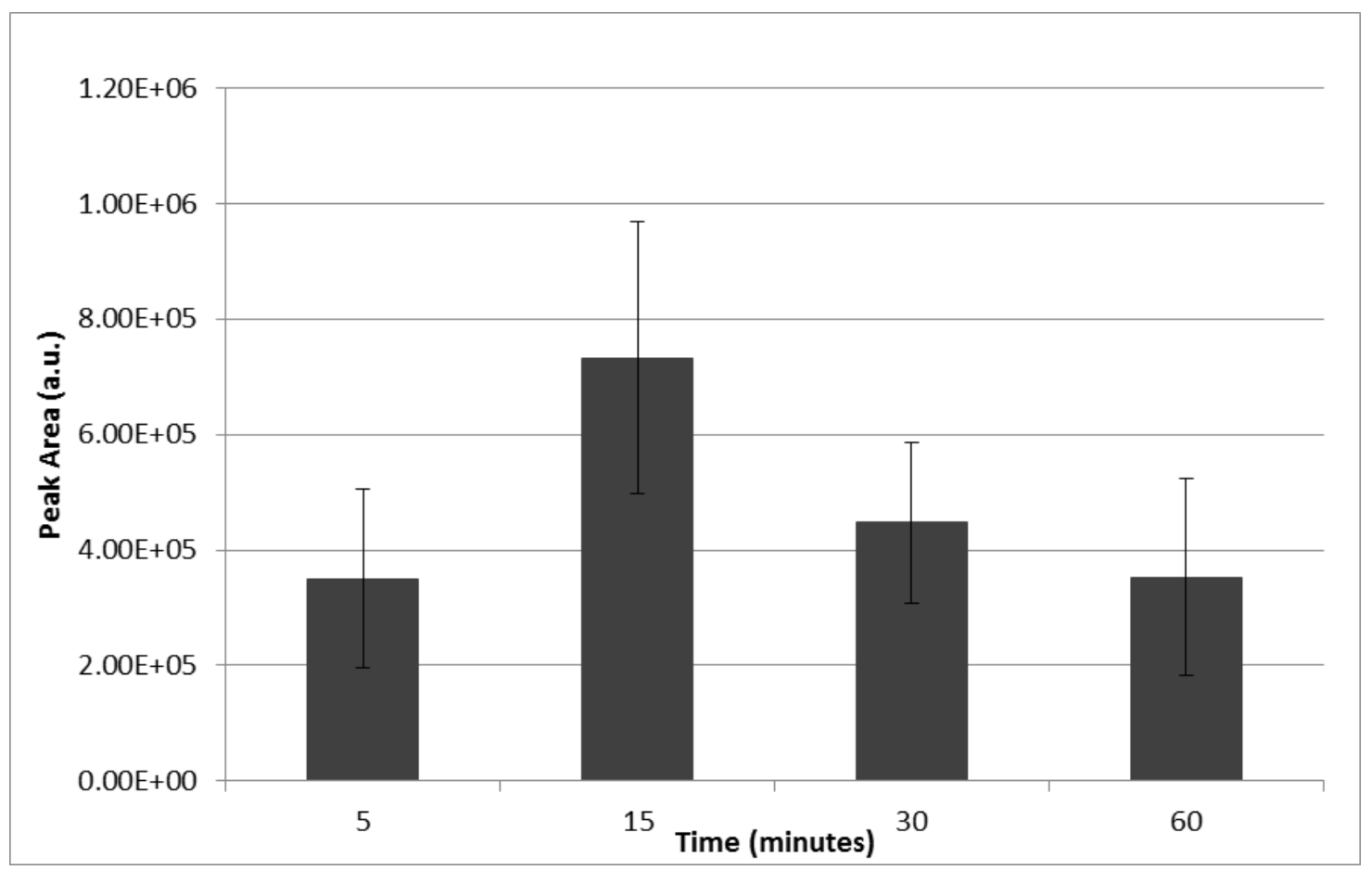

Figure 59. Reassessment of Various Equilibration Times Established for Development of the Optimal Synthetic Cathinone COMPS

From the times examined it was indicated that the length required for complete equilibration to occur within the system remained at 15 minutes. By Shortening (5 minutes) and prolonging (30 and 60 minutes) the equilibrium times around 15 minutes there was a decrease in the amount of odorant detected. Although, statistical testing indicated that there was no significant difference between the equilibration times with an F calculated value of 3.0601 and f-critical value of 4.0662 (ANOVA); 5 minutes suffered from reproducible issues. Therefore 15 minutes remained the optimal equilibrium time used for the examination of all COMPS.

After the equilibration was chosen, the length of fiber exposure was reexamined to determine if a shorten extraction time would still prove beneficial. $10-\mathrm{mL}$ vials 
containing both the gauze pad and the $100 \mathrm{ng}$ of odorant were created and equilibration was held for 15 minutes followed by varying extraction times: 1, 15, 30, 60, and 90 minutes. Extraction times of 15 and 30 minutes were initially examined to determine the total amount yielded. Afterwards the times were shorten to 1 minute and extended to 90 minutes. As expressed in figure 60, an exposure time of one minute was eliminated as a diminished quantity of the odorant was extracted; therefore the initial hypothesis that one minute was the optimal extraction time was proven false.

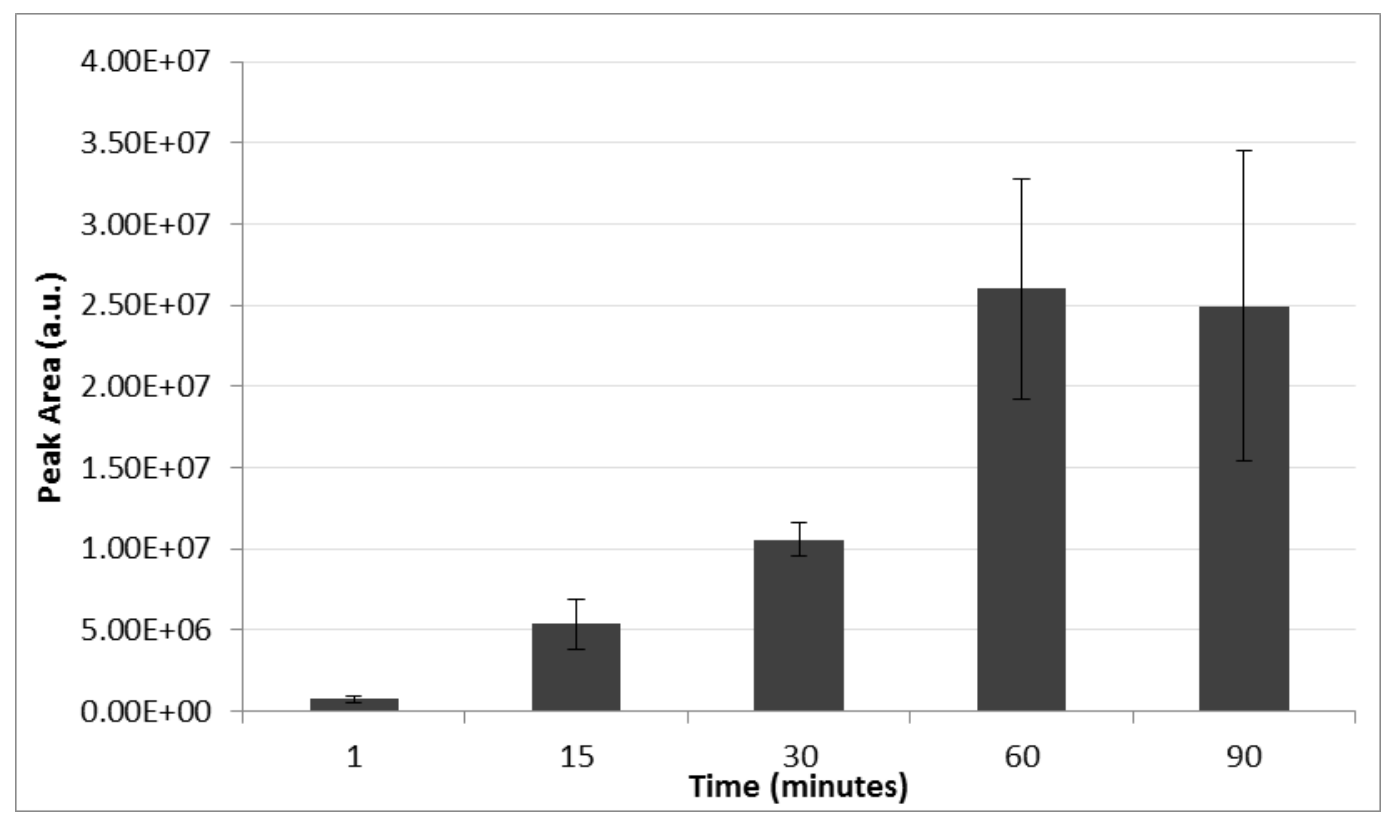

Figure 60. Reassessment of Various Extraction Times Established for Development of the Optimal Synthetic Cathinone COMPS

Although similar abundances were obtained from increasing the extraction time from 15 to 30 minutes, overall there was a slight increase in odorant detected. The largest increase experienced was observed by doubling the exposure time from 30 minutes to a full hour. When comparing the amount of odorant extracted from an exposure time of 60 
minutes versus the lower extracted times, the difference detected was proven to be statistically different $(\mathrm{F}$ value $=29.2352 ; \mathrm{f}$ critical $=4.0662)$. The extraction cycle was repeated again with an allotted time of 90 minutes, to note any visible change in abundance detected. Figure 60, shows the comparison of between the extraction times of both 60 and 90 minutes. Although the figure depicted a slight decrease in the abundance detected; statistically testing proved that there was no significant difference in the extracted quantity $(\mathrm{F}$ value $=0.023464)$ when the exposure time was lengthened. However, the extraction length of 90 minutes did suffer from a larger RSD value of approximately $40 \%$. No other times beyond 90 minutes were examined after the downward trend in detection was experienced from this sampling parameter. It was determined that optimally decreasing the initially extraction time from 4 hours (for odor profiling) to 1 hour (for odorant detected) proved a viable option for the maximum yield of the odorant within the COMPS delivery system.

\subsubsection{COMPS: Externally Sampled Internal Standard (ESIS) Optimization}

An externally sampled internal standard was selected to account for the environmental fluctuations that could be encountered when analyzing the life span of the optimally created COMPS. Traditional use of an internal standard, places the standard within the matrix of the sample to undergo the same exertion. When developing a COMPS system the insertion of an internal standard within the bag was not feasible as the bag was to contain only the odorant. In efforts to address this, an externally sampled internal standard was used. Prior to headspace collection of the COMPS, the fiber would be exposed to an internal standard. Selection of the appropriate standard was conducted 
by choosing a compound that was structurally similar to the active odorant and possessed a similar vapor pressure. Through consideration, piperonal was chosen as the internal standard for optimization. Undergoing asymmetrical extraction required assessing an equilibration and extraction length separate from that chosen for the COMPS. Varying amounts of piperonal were placed inside of a $10-\mathrm{mL}$ vial and headspace was performed for 15 seconds for both the equilibration and extraction. Pure versus diluted samples were compared to determine which gave a relatively high yield of the standard without overloading the fiber. In total 0.001 grams of piperonal was placed inside a $10-\mathrm{mL}$ vial to determine the equilibration.

For the equilibration trials $0.5,1,5$, and 15 minutes were assessed for development of an optimal headspace. After assessment it was determined that the $10-\mathrm{mL}$ required one minute for the internal standard to reach equilibrium. Decreasing equilibration to 30 seconds was not viable as there was a significant decrease in the abundance detected; while being unreproducible. Increasing the length for equilibration longer than a minute provided no increase in the detection of piperonal, beyond what was obtained for the first minute (F calculated: .02043; f critical: 5.1433). It was determined that applying this setting allowed the maximum amount of volatile in the headspace for extraction and was therefore selected. 


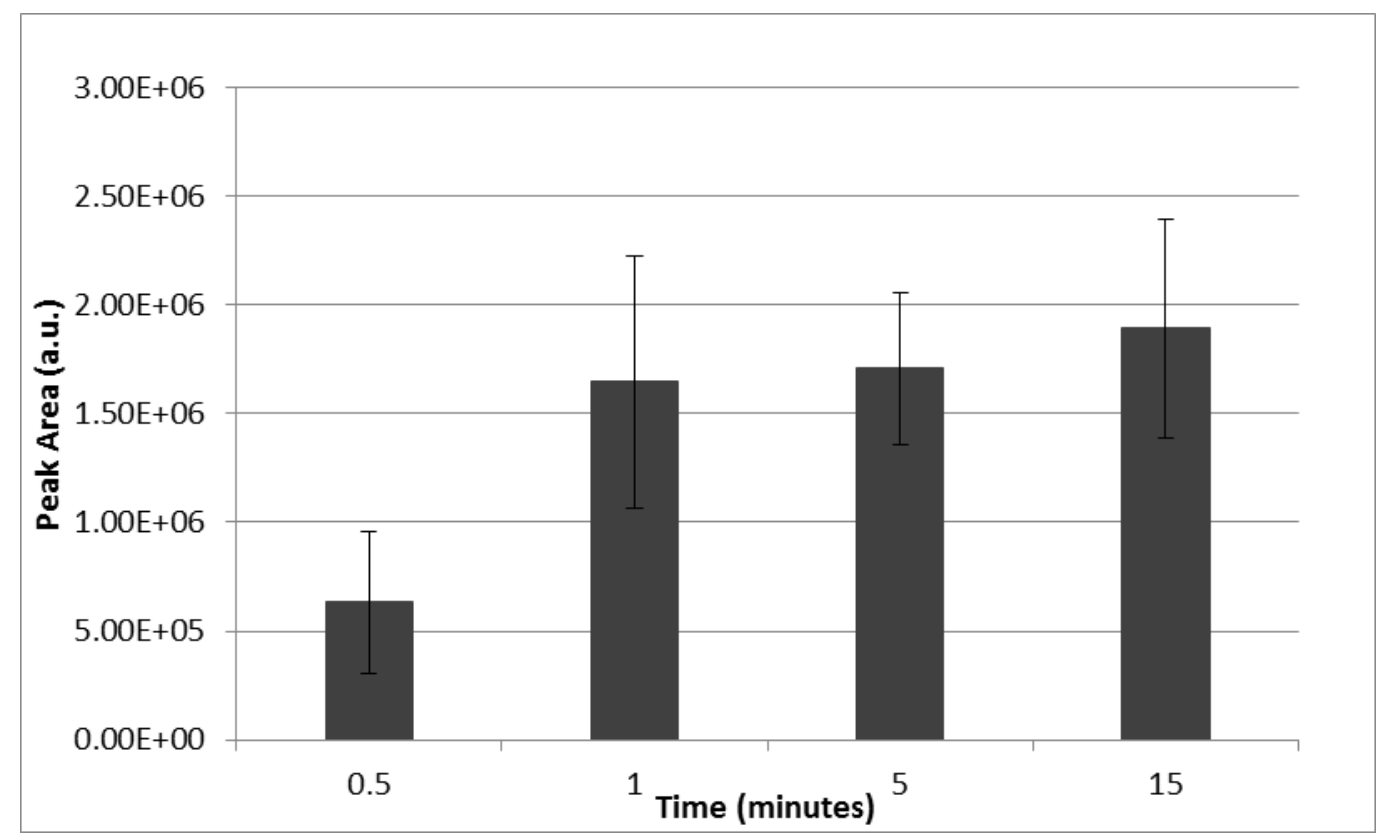

Figure 61. Assessment on the Effect Varying the Equilibration Time has on the Extraction of the Externally Sampled Internal Standard (ESIS)

The extraction of the externally sampled internal standard was performed by applying the newly established equilibration time and $100 \mathrm{ng}$ of the active odorant placed in a vial as described previously (during SPME optimization). The goal was to test various extraction times followed by the optimized extraction of the analyte to determine the appropriate extraction of the internal standard. The ratio of the analyte and standard were compared until an extraction time resulted in a stabilized ratio. The extraction times compared were $1,5,15,22,30$, and 60 seconds (figure 62). 

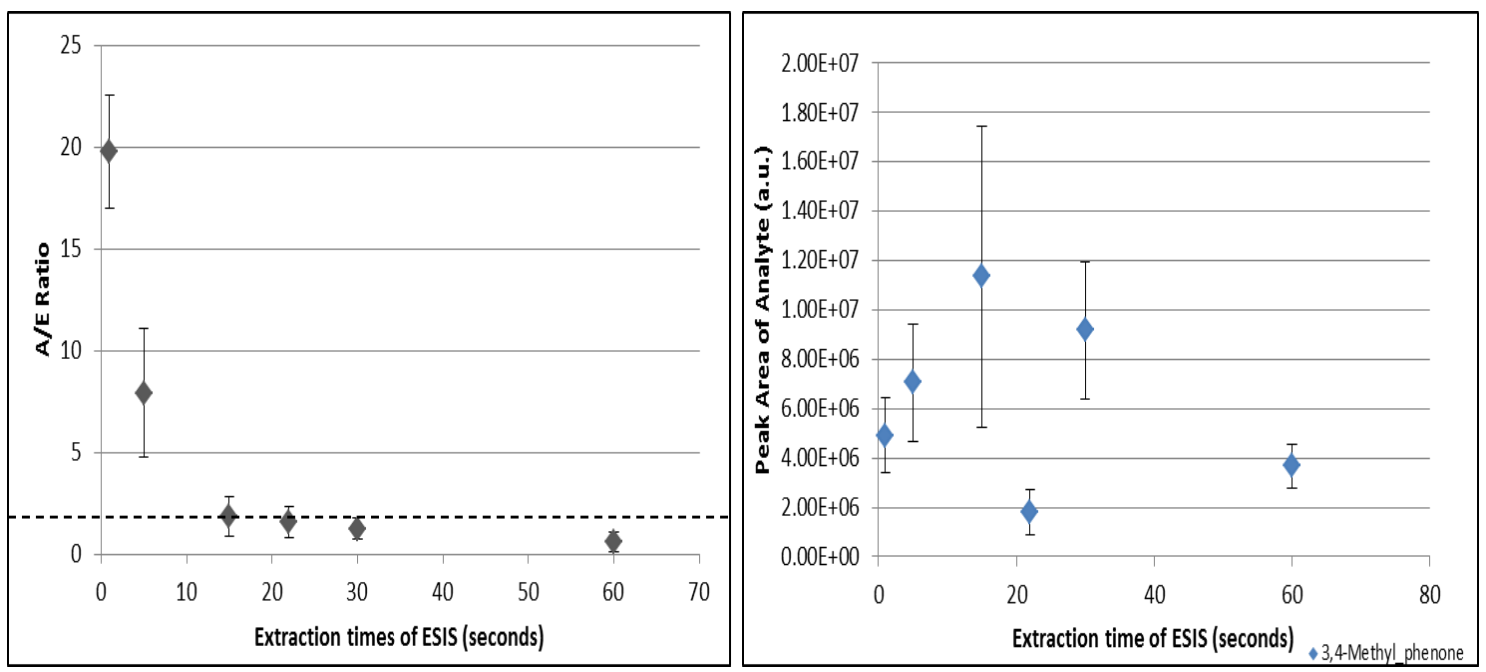

Figure 62. Selection of the Optimal Extraction time for the Externally Sampled Internal Standard based on its Effect on the A/E Ratio versus Solely Observing the Analyte

The lowest extraction times of 1 and 5 seconds were initially eliminated as the ratios were very large (19 and 8 respectively). The length of times did not allow for a sufficient amount of the internal standard to be extracted prior to introduction of the analyte. In addition, there was a lot of variation that took place when the standard was sampled for these times, resulting in large fluctuations. When the exposure was conducted for 15 and 30 seconds the ratio stabilized at approximately 1.8 and 1.3. By increasing the extraction to 60 seconds there was a decrease in the ratio. Revisiting the 15 and 30 second extraction times, 22 seconds was tested to determine if any length between these variables would also result in a ratio close to what was achieved for 15 and 30 seconds. Analyzing the abundance of ESIS and analyte extracted the corresponding ratio was approximately 1.56 . The $\mathrm{A} / \mathrm{E}$ ratio remained stable between extraction times of 15 and 30 seconds. By selecting the medium, 22 seconds, a relatively good yield for both the 
internal standard and active odorant were achieved without competing for adsorption/absorption on the fiber.

\subsubsection{COMPS: Absorbent Optimization}

For the controlled rate of dissipation for a COMPS to be actively employed, the material used to bind and maintain the odorant over an extended period of time must be tested and established. For this study, three different types of absorbent were tested: surgical gauze, cellulose, and alumina.

Alumina $\left(\mathrm{Al}_{2} \mathrm{O}_{3}\right)$ is a fine, grained substance that bears the physical and molecular structure in figure 63. As a fine white powder, this absorbent contains an aluminumoxygen bound structure; bearing exposed oxygen groups for interaction. Typically used in column chromatography, alumina is a nontoxic absorbent that does not swell in water, possesses a moderate thermal conductivity, and is currently used to separate compounds varying polarities based on their interaction with the absorbent. 

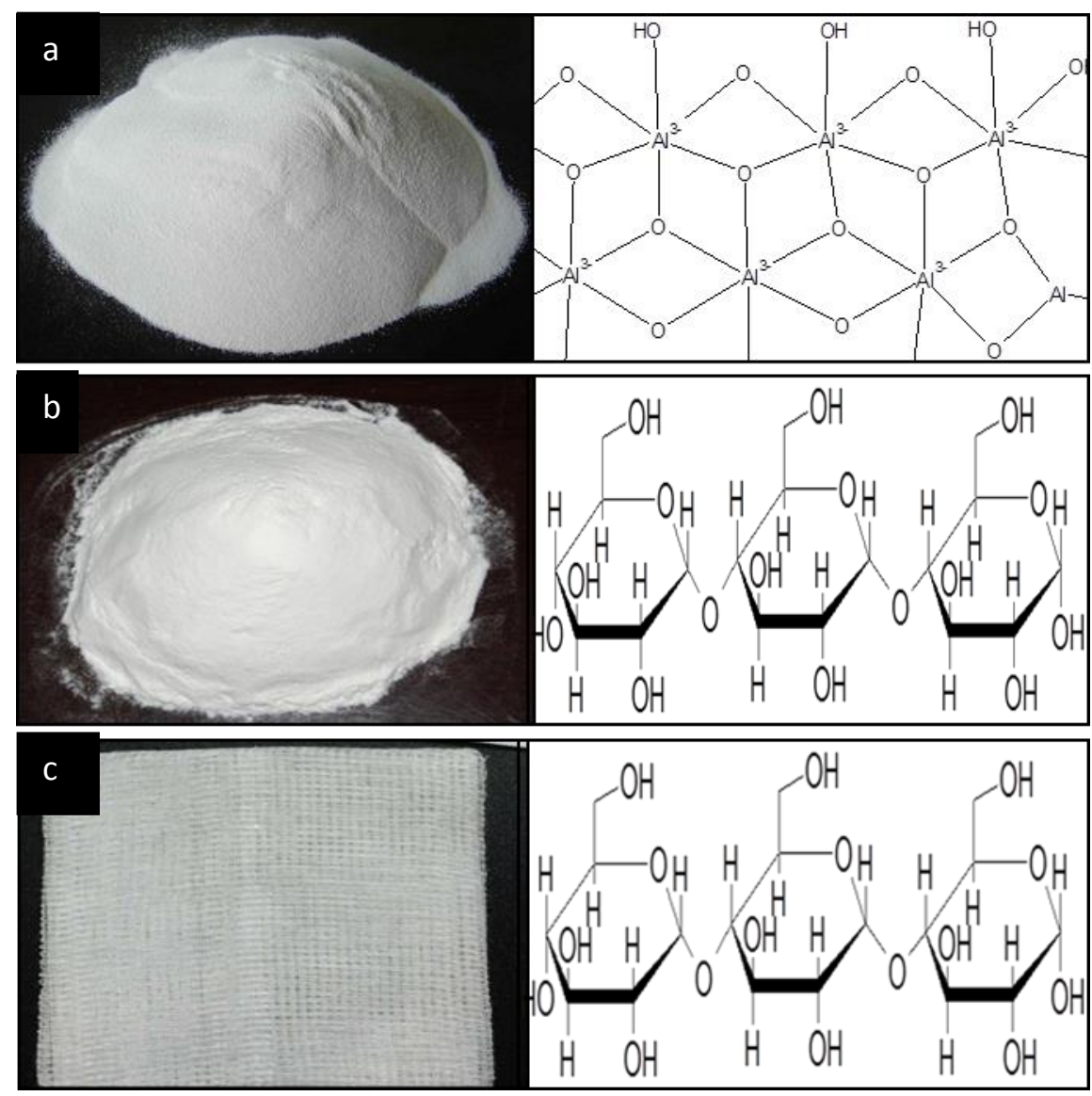

Figure 63. The Physical and Molecular Depiction of Various Absorptive Materials (a) Alumina (b) Cellulose and (c) Gauze Pad

Cellulose is a light weight $20 \mu \mathrm{m}$ powder that is very airous. The molecular structure of this material, shown in figure 63, contains several d-glucose molecules connected by an oxygen group forming a long polysaccharide chain. The molecules are connected for the formation of a free range loose powder. The surgical gauze tested during this study was continued from the odor delivery system implemented during the imprinting and testing process. The pad was built by weaving cotton fibers in a crisscross pattern. In regards to the cotton fiber, more than $85 \%$ of the substance is contributed to cellulose (as seen with the powder form tested). However the remaining constituent of the 
fiber is contributed to other non-cellulosic polysaccharides; which can include fats, waxes, proteins, and more. Although the gauze pads contain additional material beyond what is observed with pure cellulose, another difference was the absorbent's configuration. The pure cellulose is unbound in the powder form and therefore has the ability to move in an enclosed volume. While, the cellulose found in the gauze was bound in a fixed crossing position along the $\mathrm{x}$ and $\mathrm{y}$ axis; woven into two layers and containing small air pockets.

These materials were selected based on studies conducted for the development of COMPS for a variety aids that deployed one of the previously described materials. In order to test each absorbent, COMPSs were created in triplicates for each. The initial and post weight (after the addition of the active odorant) was recorded for monitoring purposes. The bags underwent headspace analysis over the course of the week and the dissipation of the odor was monitored over time (figure 64). 


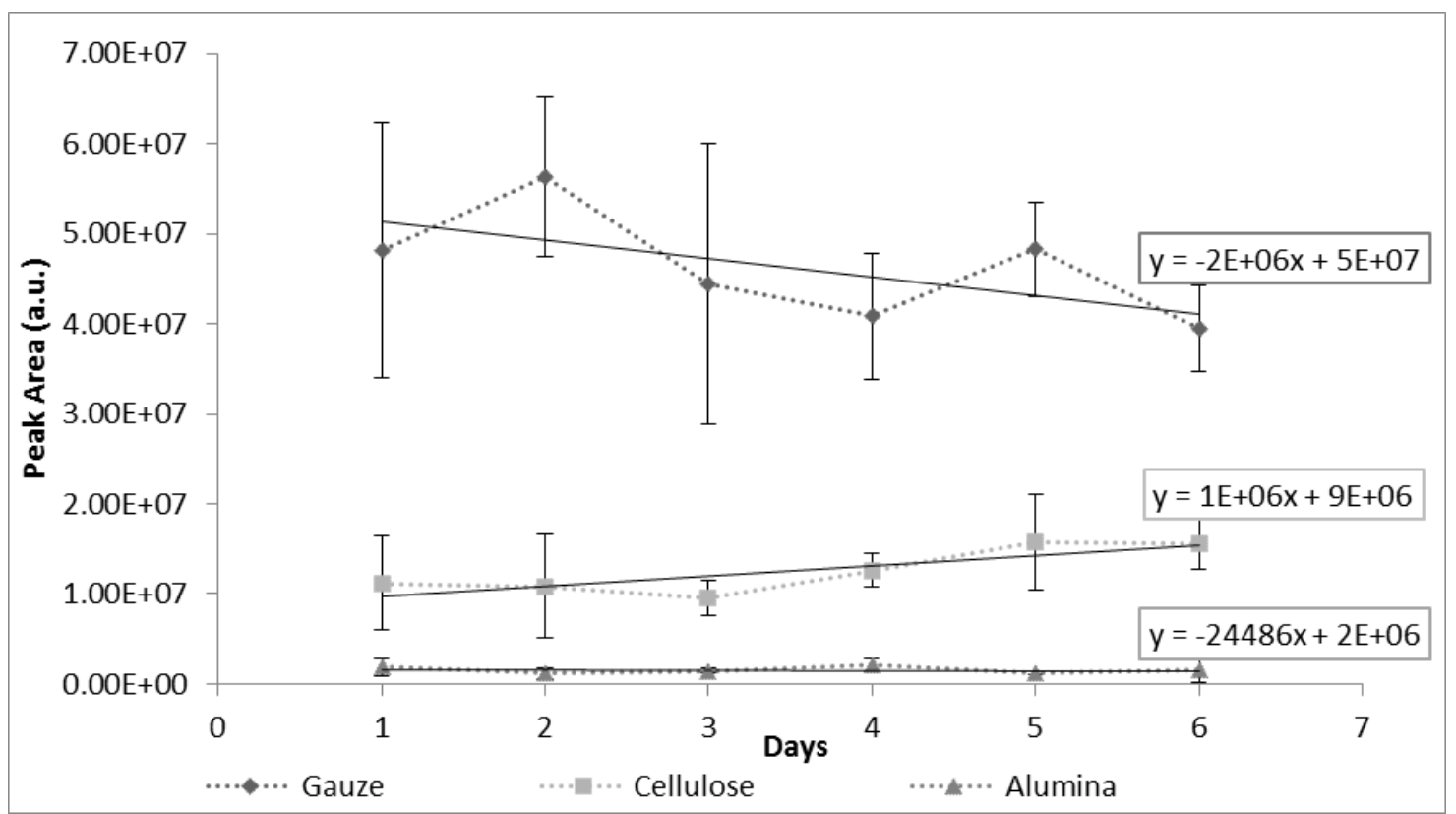

Figure 64. The Effect of Varying Absorbent Material on the COMPS Odor Dissipation

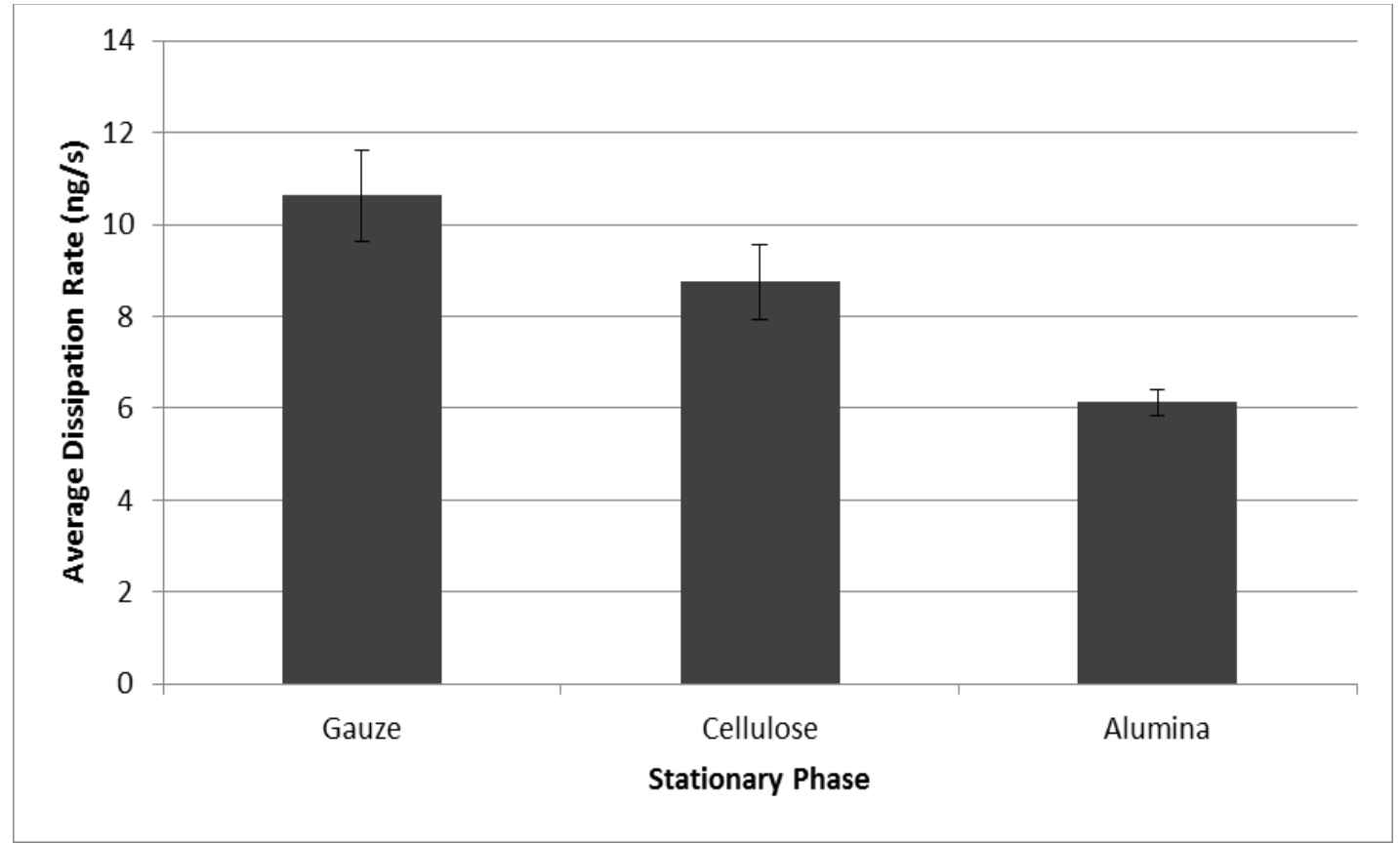

Figure 65. The Dissipation Rate of Various Synthetic Cathinone COMPS Containing Different Phases 
Based on the analysis of the COMPS over the first week the first absorbent to be eliminated was the gauze pad. This absorbent material had the largest release of odorant seen over the seven day period. In addition, this material suffered from reproducibility and precision issues caused by a lack of affinity to the odorant and environmental influences. As a primary factor for the creation of COMPS is the controlled rate of dissipation, the large fluctuation observed when using this material resulted in its elimination as the ideal candidate to optimal bind and hold the odorant for an extended period of time.

Between the remaining absorbent materials, cellulose and alumina, it was concluded that the best absorbent for retaining the odorant was cellulose. Although both absorbents had a significant lower detection in comparison to the gauze pad (F critical: 26.66; f calculated: 5.14); the odorant retained by the alumina proved quantifiably problematic with the instrumentation used. Analysis of the material overtime revealed that the odorant extracted was relatively low; comparable to the background noise produced. In summary the detection limitations encountered when using alumina, proved problematic with establishing the dissipation and evaluation of the COMPS overtime. Cellulose was able to stabilize the abundance detected by increasing the interaction and binding with the powder. Although the linear relationship showed a slight increase there was no significant difference in the odorant detected between the days. In addition, the cellulose mixture was still quantifiably measured within the software. Although both the gauze and pure powder consisted of cellulose, the increase in retention for the powder can not only be explained by the fact that it contains purely cellulose unlike the pad. Yet, the powder formation of the cellulose presents the molecules with a larger surface area 
and therefore more sites for interaction. Unlike the gauze pads (fixed in a planar structure), the powdered cellulose was allowed to engulf the active odorant, thus creating a stronger bond with the vehicle and decrease the amount detected overtime. Offering good reproducibility, cellulose was selected as the optimal absorbent to bind with the odorant and slow the dissipation rate down to a considerable level.

\subsubsection{COMPS: Polymer Thickness Optimization}

Evaluation of the absorbent material was conducted with the use of a 2 Mil Low Density Polyethylene Bag (LDPE). Ideally used for the creation of various commercialized COMPS, LDPE, as the name refers, is a polyethylene which has a slightly lower mass than volume. As an ideal permeable bag for the release of any odor, LDPE has a branched molecular system which allows a certain percentage of the odor stored inside to emit through. While being well documented as the ideal type of polymer to use for COMPS development, the thickness of the polymer can vary given the type of odorant stored within.

When referencing the thickness of a bag, the measurement of Mil is a unit for thickness in a thousandth of an inch; therefore 1 Mil means the bag possesses 0.001 inch thickness. In order to test the odor dissipation, the COMPS's polymer thickness was varied from 1.5, 2, and 4 Mil. By increasing to a 4 Mil thickness (0.004 inches), the bag was not only slightly thicker than the 2 Mil, but also possessed a stronger, more durable

plastic material used to house any heavy weight substance. The opposite can be observed, when the bag's thickness was decreased to 1.5 Mil. Although the material housed would 
contain the same weight, the fluctuation of the permeable bags was gauged to determine whether a slight modification would affect the odor delivery negatively.

In regards to the creation of each COMPS, the mixture was kept constant while the thickness was modified and developed in triplicates (including blanks). The bags were monitored gravimetrically over the course of a week and environmental factors (temperature and humidity) were recorded. Comparison of the bags was determined by examining the effect the thickness had on the permeation of the odorant stored inside (figure 66).

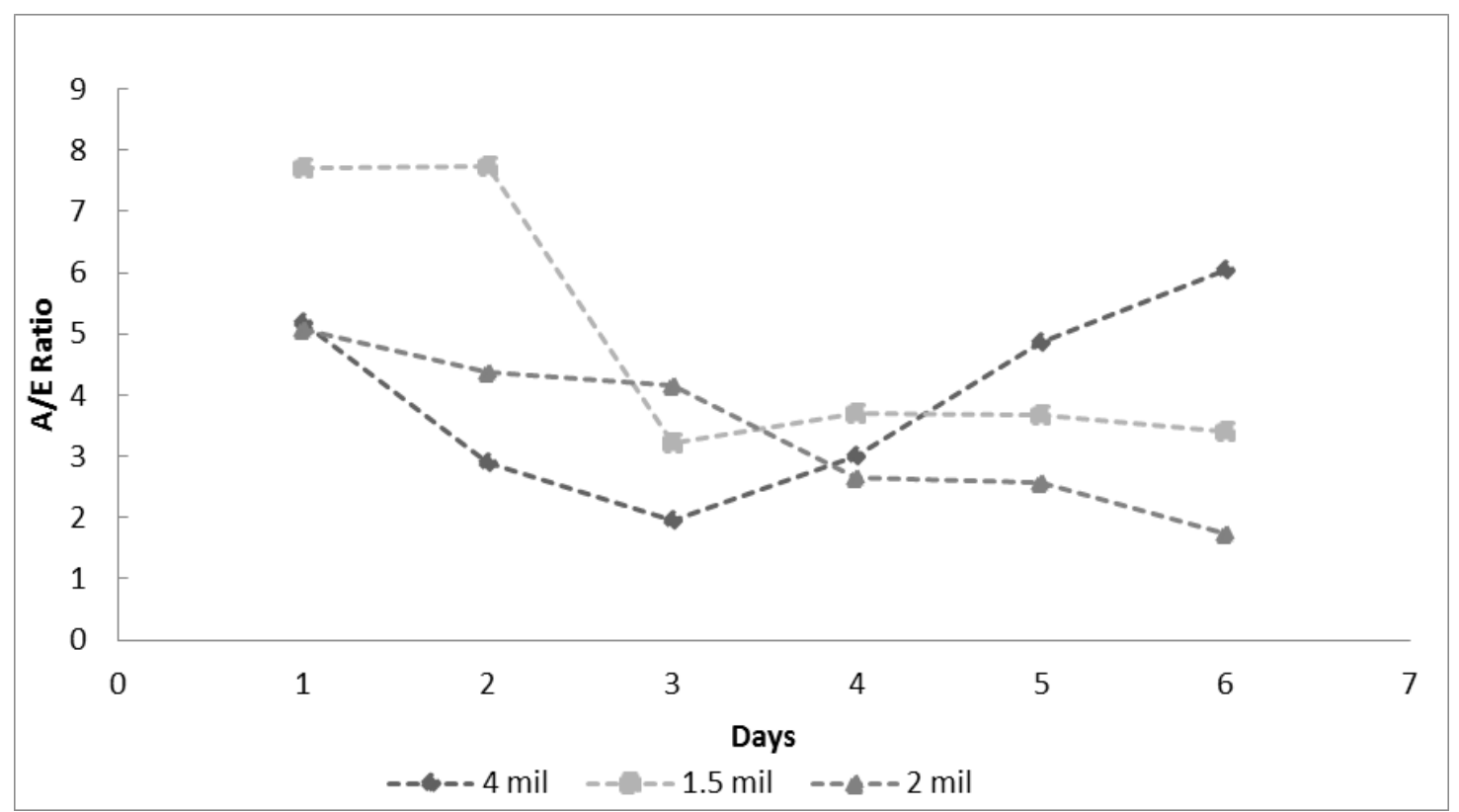

Figure 66.The Effect of Varying the Polymer's Gauge on the COMPS Odor Dissipation

During the first two days of analysis, the $1.5 \mathrm{Mil}$ bags produced the highest degree of extractable odor. The increase in abundance was the result of a thinner bag which allowed for a larger permeation of the odorant. After the first two days there was a 
substantial decrease in the amount detected before stabilization occurred. It was determined that employing a polymer gauge of 1.5 Mil would only produce a relatively steady amount of the odorant after the first two days. With the 4 Mil bags there was a significant amount of deviation that occurred over the course of the first week. The bags initially gave a good abundance although lower than both the 1.5 and 2 Mil bags. This was expected as the bag incorporated a slighter thicker material, thus holding the odor in more.

However as evaluation continued the odor released continued to decrease overtime, as expected, yet by the fourth day the abundance detected began to increase. This increase was explained by the increase in temperature and humidity during extraction, figure 67 . As noted from this figure, during the fourth, fifth, and sixth day of sampling the temperature and humidity had increased from when the bags were initially created. This increase in detection was explained by the elevated environmental parameters experienced during those days which caused an upward shift in dissipation. While the other bags were still able to release the odor, the 4 Mil bags retained the volatile longer, resulting in an increase in detection. Overall, the 4 Mil bags were largely influenced by the environmental factors in which it was stored. 


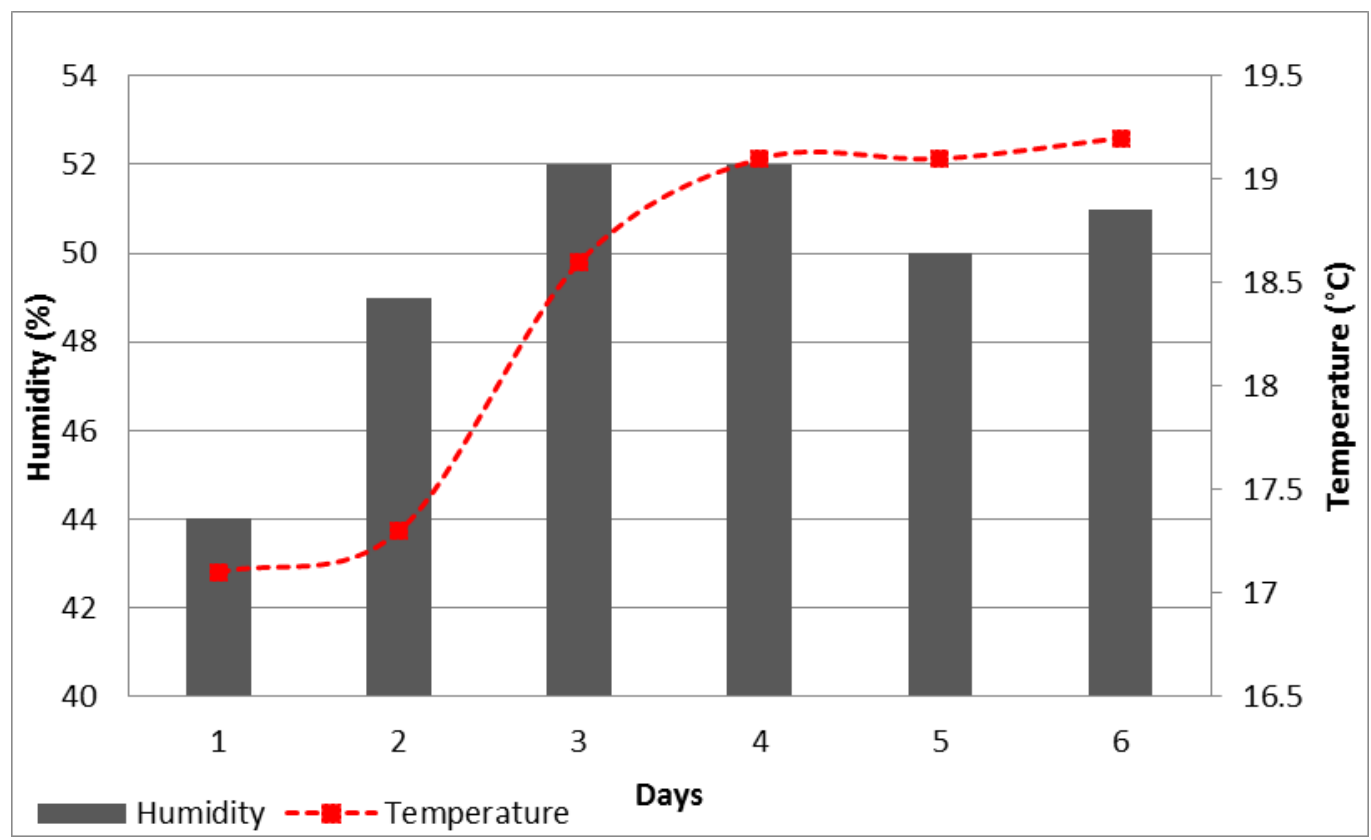

Figure 67. Temperature and Humidity Recordings during Optimization of the COMPS Polymer Gauge

The 2 Mil bags were optimally selected for housing. Unlike the other thickness, this bag was able to produce a sufficient abundance of the active odorant. In addition, this bag seemed to be uninfluenced by any of the environmental factors encountered, and progressively decrease its abundance overtime as expected. Although the 0.0015 inch gauge did display a downward trend, the 0.002 inch bags were more successful at establishing stability. Over the course of a month, the 2 Mil bags with the optimal mixture were examined to determine the minimal life span of the given aid. Through experimentation it was determined that the projected lifespan of the 2 Mil LDPE training aid containing a mixture of 3,4-Methylenedioxypropiophenone and Cellulose would remain stable and continuously permeate for a total of 25 days. This shelf life was determined by the COMPS freely suspended in an open environment, with daily 
interaction with various uncontrollable factors. The calculated life span of the COMPS would be extended once the aid was stored in a non-permeable container, in a dry location, and at stable temperatures until placed in the field for use.

\subsubsection{COMPS: Containment and Validation}

There are two essential components to developing a training aid in the form of COMPS: (1) the selection of a permeable polymer bag and (2) the selection of a nonpermeable outer bag. Permeability of the inner bag is used to permit the release of an enclosed odor. However, as the odor flows outward, the same phenomenon can occur with any outward vapors or contaminates that may flow into the bag. Selection of an optimal non-permeable bag was done for two reasons. The first was for a protective barrier. The outer bag acts as a barrier and stops the binding of any containment (not contributing to the training aid) to the absorbent; therefore contaminating the inner bag. The second purpose of the non-permeable bag was to establish equilibrium within the system. As the permeable bag remains closed inside the outer, equilibrium is established within the system; thereby preventing the continued loss of the compound bound to the absorbent. In addition once the bag was used, placed back in its appropriate container, equilibrium would be reestablished.

To assess the appropriate container required for these two factors, an aluminum ziplock bag was tested. In total six COMPS were created solely containing the absorptive material. The initial weights of all the blank bags were noted and three of the bags were placed inside the aluminum bag, while the remaining three were openly suspended on a metal netting wire within a tank. The contaminate used during testing was the active 
odorant of cocaine, methyl benzoate. This compound was utilized as the contaminate because ideally all narcotic COMPS would be stored in close proximity, therefore the first route of possible contamination would be from any neighboring COMPS bags. In total, 1 gram of methyl benzoate was placed inside a beaker and stationed in the glass tank equidistant from both the freely suspended bags and the alumina bag housing the remaining blanks. Overtime, the bags were weighed and SPME headspace was performed to determine the amount of contaminate present within each bag.

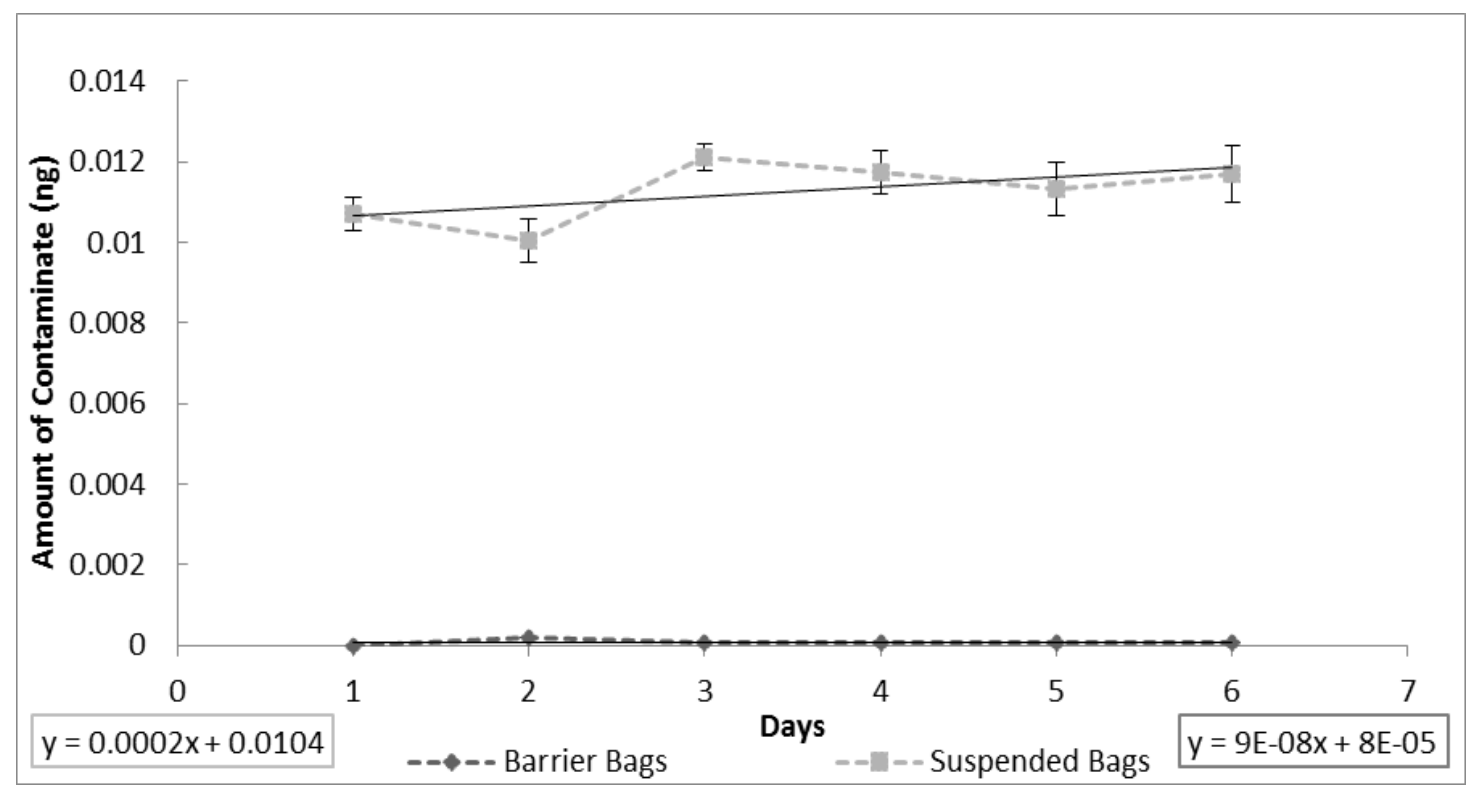

Figure 68. Assessment of the Aluminum Bag as an Efficient Barrier Bag between the Permeable Bag and the Surrounding Atmosphere

As depicted in figure 68, the bags which were freely suspended in the tank did contain methyl benzoate. As hypothesized, the polymer bags not only permitted the release of any contained odorant, but also allowed surrounding vapors to migrate through the polymer surface and bind with the absorbent. Although the 2 Mil LDPE bag was 
successfully used for the permeation of the active odorant, it failed to efficiently keep out any surrounding contaminates present within the surrounding headspace. Analyzing the blank aids stored in the aluminum bag showed that the ziplock container was capable of housing the odor while acting as a barrier to block the migration of any surrounding vapor into the permeable bag. It was determined through experimentation that the aluminum bag would be an efficient buffer and equilibration tool for the housing of the COMPS for synthetic cathinones.

Lastly, was the validation of the developed COMPS for accurate detection of synthetic cathinones. This study was assessed in a controlled experiment and within a preliminary field evaluation. Initially the canines were provided with the developed COMPS and allowed to imprint on the aid. After 1 month, a validation study was performed to test that the canine was successfully imprinted for the detection of the odorant; 3,4-methylenedioxypropiophenone. The canine underwent four validation trials containing one type of the cathinone derivative. The validation trial for the canine showed a combined total alert rate for the odorant and bath salt cases to be $100 \%$ and $96 \%$ respectively. The alerts observed within the trial supported the working concept that the odorant was optimally selected for the canine's positive indication.

Preliminary field evaluation of the COMPS was performed for the canines that were imprinted on the COMPS but were not able to test on any cathinone sample due to accessibility issues. In addition field observations were performed to track and record how well the canines alerted to synthetic cathinones containing a different profile; in 
comparison to the four cathinone derivatives continuously used. Three canines were imprinted and validated on the synthetic cathinone COMPS initially.

When any given material was confiscated from an individual during a routine traffic stop, the evidence was submitted to the crime lab with a case report. This report indicated what the officer encountered in the field and what they believe the substance submitted to evidence contained based on field testing and physical traits. Within a case report, any valuable information deemed necessary is added to the report to aid in the assessment of the case. With this in mind the canine's detection capabilities were examined over time. Each handler was assigned a different locator number. When the canine handler encountered a substance presumptively identified as a type of bath salt or possessed a physical characteristic different than previously encountered drugs, the canine team's locator number was placed on the report before transition to the crime lab. Once instrumental analysis of the case confirmed the presence of any bath salt, the canine locater was recorded over time to monitor the successful alert of any bath salt after successful imprinting on the pseudo. In addition, the canines were continuously tested on the 3,4-methylenedioxypropiophenone to ensure that odor recognition remained intact. Over the course of four months the canine teams were monitored, the field detection was recorded, table 31, and used to support the selection of the odorant for the creation of the mimic for synthetic cathinones. The preliminary evaluation conducted also supported the selection of 3,4-methylenedioxypropiophenone as the odorant required to detect not only one type of synthetic cathinone, but a wide range. 
Table 31. Preliminary Field Surveillance of Detection of Synthetic Cathinones using Biosensors Imprinted on a Pseudo COMPS

\begin{tabular}{|c|c|c|c|c|c|c|}
\hline $\begin{array}{c}\text { Canine } \\
\text { Team }\end{array}$ & Ethylone & MDPV & $\boldsymbol{\alpha}$-PVP & Cocaine & Naphyrone & $\begin{array}{c}\text { Total No of } \\
\text { Bath Salts } \\
\text { Detected }\end{array}$ \\
\hline $\mathbf{0 7 2 3 1 7}$ & $\mathrm{XX}$ & $\mathrm{X}$ & & $\mathrm{XXXX}$ & & 3 \\
\hline $\mathbf{0 7 1 9 4 6}$ & & & & $\mathrm{XX}$ & & 0 \\
\hline $\mathbf{0 7 1 9 8 6}$ & $\mathrm{XX}$ & & $\mathrm{X}$ & & $\mathrm{X}$ & 4 \\
\hline
\end{tabular}

Development of a synthetic cathinone COMPS was the primary focus successfully concluded within the final task of this dissertation. In efforts, to create the most ideal pseudo, various parameters including the binding absorbent, the polymer gauge, and containment of the aid were all assessed. Through experimentation, this task was able to determine that the optimal absorbent for release of the active odor was accomplished when cellulose was mixed and stored inside of a 2 Mil thickness LDPE bag. Not only was the dissipation of the odor slowed, but the bag's permeation rate was able to withstand the temperature and humidity fluctuations in the surrounding environment. The last component assessed for this task not only proved that the pseudo was successfully created, but monitoring the canines over a large span of time supported the correct selection of the odorant used to create the pseudo. This mimic training aid showed that once imprinted canines could accurately detect a variety of synthetic cathinones within the field. 


\section{Conclusion}

To date, there have been few analytical studies conducted for the analysis of synthetic cathinones. Furthermore, there are no studies currently exploring the characteristic aspect of these drugs in a non-destructive manner, which can be applied to developing a solution to the evasion of these drugs due to lack of detection. Therefore there were two goals of this dissertation research, the first being to develop an analytical method which could be used for the development of VOC profiles (characteristic odor profiles) used to identify and distinguish the difference between generational synthetic cathinones. The second goal of this dissertation was to assess the current detection capabilities of narcotic detection canines and develop a safe mimic for the detection of newly synthesized cathinones.

Initially a method optimization study was performed to determine the most ideal parameters required for successful examination of these drugs. Through experimentation it was determined that using a smaller volume container (4-mL vial) to house one gram of the substance was an efficient amount for detection of the volatiles; unlike the larger containers. Through evaluation it was determined that undergoing analysis was ideal when separation was performed on a polar sol-gel wax column. While analysis also revealed that positive identification required the use of soft ionization to preserve the molecular structure lost during electron impact ionization; by employing methanol as the chemical ionization reagent, software settings for storage level, ejection amplitude, background mass, target ion count, maximum ionization time, and maximum reaction 
time were established as the parameters required to detected both methylone, MDPV, and other closely related synthetic cathinones.

VOC profiling of the synthetic cathinones was achieved through headspace analysis performed by solid phase microextraction. Preliminary results showed that employing a polydimethylsiloxane-divinylbenzene fiber chemistry was ideal for the extraction of a wide range of compounds in comparison to the other chemistries. Through experimentation the optimal parameters used to enhance the fiber to volatile interaction was maximized by examining an increased in 1,3-Benzodioxole, 4-Bromo-1,2(methylenedioxy)benzene, 2,4-bis(1,1-dimethylethyl)-phenol, 3,4Methylenedioxypropiophenone, and 4'-Ethoxy-2'-hydroxyacetophenone when the system established equilibrium at 15 minutes and the fiber was exposed to the headspace for 4 hours. Observation of the volatiles also revealed that performing the extraction under a temperature of $50^{\circ} \mathrm{C}$ provided a larger abundance and quantitative of compounds detected; followed by a desorption of fifteen minutes within the gc inlet of the instrument.

Applying the optimized extraction to analyze sixteen different synthetic cases, proved vital in understanding the molecular components found within these drugs. Through the developed method the parent drugs of methylone, ethylone, 3,4methethylone, and $\alpha$-pvp were identified . Developing the headspace profile highlighted that although the parent drug is responsible for the euphoric effect the compound is not abundantly found within the headspace of the drug. Therefore it does not largely contribute to the odor encountered with these synthetic drugs. Headspace analysis also 
revealed that a large component of the odor profile developed did result in the molecular breakdown of the parent drug. Typically with the loss of the alkyl chain that was present.

Headspace analysis was not only successful at extracting the parent drugs for identification and other smaller constituents. For the first time in the analytical studies conducted with cathinone derivatives the odor profile could be used to shed some light on the synthesized reactions undergone to create these drugs. The molecular information obtained within this dissertation is the first on the analytical realm to provide characterizing molecular information in regards to synthetic cathinones, while using confiscated unaltered samples and maintaining the non-destructive nature.

Furthermore, assessment into the detection capabilities of currently deployed narcotic canines revealed that the large number of cathinones derivatives currently smuggled into Florida were able to bypass our number one field detection instrument; canines. More than $85 \%$ of the canines were not able to detect any of the cases presented; while $20 \%$ only showed interested after being prompted by the handler. With the odor profiles developed, extending the detection capabilities of the currently deployed canines to include these new synthetic drugs was achieved. With the restriction of transporting any illicit substance and the potency of these drugs when accidental exposure occurs; the need for a mimic was essentially the overall goal of the second half of the dissertation. In order to determine the odorant responsible for the detection of the synthetic cathinones, seven canines had to be imprinted on the illicit material. From the odor profiles developed the compounds were divided up and singular and binary mixtures were given to the canines to determine the active odor. 
Odorant COMPS created were exposed to the canines and the canines showed the greatest interest in the odorant, 3,4-Methylenedioxypropiophenone; $80 \%$ and greater for the K-9s imprinted on $\alpha-p v p$ and more than $90 \%$ for the canines imprinted on ethylone. The canine also showed a slight interest in the odorant, ethyl benzoate; $90 \%$ for the canines imprinted on $\alpha$-pvp and $40 \%$ for the remaining canines. In order to test whether the canines would have a greater response to a mixture of these compounds, a binary mixture was created. This mixed introduction of odors resulted in a decrease in response. Through various canines trials it was determined that 3,4-methylenedioxypropiophenone was the odorant responsible for a positive alert for detection of both ethylone and $\alpha-p v p$. Through canine trials odor generalization was established with this compound allowing for the detection of its present in various bath salts and in derivatives bearing similar molecular compounds. This odorant proved to be an ideal pseudo for the detection of not just one class of synthetic cathinones, but multiple.

The last goal of this dissertation focused on developing an odor mimic in the form of a COMPS to be delivered to various detection teams. Experimentation showed cellulose to be the most ideal absorbent as the gauze pad's dissipation rate was too high and fluctuated too much during interday analysis. An externally sampled internal standard was used to determine the appropriate thickness of the permeable bag to reduce the rate of dissipation and was determined that 2 Mil LDPE bags were ideal for storage of the odorant. Assessing the storage of the permeable bag proved that placing the bag into a non-permeable aluminum bag was a sufficient barrier and aided in the equilibration. Validation of the working COMPS displayed the successful extension of the narcotic 
detection of canines by allowing the teams to successfully add synthetic cathinones to the list of narcotics identified and detected within the field.

The results from this research have aided the scientific community in two ways. This study has provided a way to characterize and identify a variety of synthetic cathinones, without chemically modifying or destroying the sample. This study has showed that although the parent drug is similar to MDMA and methamphetamine, the VOC profiles of these drugs are different. In addition this dissertation has assisted with overcoming the detection issues deployed canine teams faced. This study utilized the learned odor profiles for a variety of drugs into determining the odorant used for detection of the synthetic cathinones. This odorant was used to train canines that currently lack the ability to detect bath salts because they are not able to obtain the material to imprint their canines, and using the illicit material has proven very hazardous. This dissertation not only proves beneficial to the scientific community but also to law enforcement. Therefore, based on these findings it would suggest that the active odor for the detection of synthetic cathinones would be 3,4-methylenedioxypropiophenone. Developed into a COMPS, this odorant was and will continue to be used to monitor the successful detection of synthetic cathinones in the field. 


\section{Reference List}

1. Frank. Khat. 2016. 7-7-2016.

Ref Type: Online Source

2. Bob Warren . Snorting bath salts pushed St. Tammany man to suicide. New Orleans The Times-Picayune . 1-18-2011.

Ref Type: Online Source

3. Christine Clarridge . Drug may have been behind the murder-suicide of Spanaway family. The Seattle Times . 4-22-2011.

Ref Type: Online Source

4. abc News . Face-Eating Cannibal Attach May Be Latest in String of 'Bath Salts' Incidents. abc News . 2016. 3-1-2016.

Ref Type: Online Source

5. Crimesider Staff . Rudy Eugene's Toxicology Report: Experts speculate on what caused 'face-chewing' attack. CBS News , 1-3. 6-28-2012.

Ref Type: Online Source

6. Huffpost Miami . Rudy Eugene: No Bath Salts, Only Marijuana Found In FaceEater Toxiciology Tests (VIDEO, PHOTOS). Huffpost Miami [US]. 8-272012.

Ref Type: Online Source

7. Imelda L.Paredes . Schedules of Controlled Substances: Temporary Placement of Three Synthetic Cathinones Into Schedule I. 76[Federal Register Notice of Intent Rules-2011], 55616-55619. 9-8-2011. Department of Justice: Office of Diversion Control.

Ref Type: Generic

8. S.R.Belenko Drugs and drug policy in merica: A documentary history; Greenwood Press: Westport, CT, 2000.

9. James A.Inciardi Handbook of Drug Control in the United States; Greenwood Press: Westport, Connecticut, 1990.pp. 1-425.

10. The Federal Bureau of Narcotics. In Handbook of Drug Control In the United States, James A.Inciardi, Ed.; Greenwood: 1990; pp 33-39.

11. Narcotic Control Act of 1956. 19-24. 1-1-1956.

Ref Type: Statute

12. Katheleen J.Frydl The Drug Wars in America, 1940-1973; Cambridge University Press: New York, New York, 2013.pp. 1-447. 
13. Joyce H.Lowinson; Pedro Ruiz; Robert B.Millman; John G.Langrod Substance Abuse: A Comprehensive Textbook; 4th ed.; Lippincott Williams \& Wilkins: Philadelphia, 2005.pp. 1-1427.

14. DEA Diversion . Controlled Substances Schedules. Drug Enforcement Adminstration , 1-14. 5-13-2016. Department of Justice. 7-12-2016. Ref Type: Online Source

15. Center for Drug Evaluation and Research (CDER) . Guidance for Industry: Assessment of Abuse Potential of Drugs. 1-25. 1-1-2010. U.S. Department of Health and Human Service: Food and Drug Administration. Ref Type: Generic

16. Dr.Steven M.Melemis . Withdrawl. Addictions and Recovery . 5-20-2016. Ref Type: Online Source

17. C.K.Himmelsbach Clinical Studies of Drug Addicition: Physical Dependence, Withdrawal and Recovery. Arch Intern Med (Chic) 1942, 69 (5), 766-772.

18. Abdullahi S.Elmi The chewing of khat in Somalia. Journal of Ethnopharmacology 1983, 8 (2), 163-176.

19. Rita Annoni Manghi; Barbara Broers; Riaz Khan; Djamel Benguettat; Yasser Khazaal; Daniele Fabio Zullino Khat Use: Lifestyle or Addiction? Journal of Psychoactive Drugs 2009, 41 (1), 1-10.

20. Office of Diverison Control: Drug and Chemical Evaluation Section Khat (Street Names: Khat, Qat, Kat, Chat, Miraa, Quaadka); Drug Enforcement Adminstration: Aug, 13.

21. Taking the Place of Food Khat in Ethiopia; The Red Sea Press: Trenton, New Jersey, 2010.pp. 1-239.

22. David Anderson; Susan Beckerleg; Degol Hailu; Axel Klein The Khat Controversy Stimulating the Debate on Drugs; Berg Publishers: New York, New York, 2007.pp. 1-254.

23. Ahmed Al-Motarreb; Kathryn Baker; Kenneth J Broadley Khat: Pharmacological and Medical Aspects and its Social Use in Yemen. Phytotherapy Research 2002, 16, 403-413.

24. What are stimulants? National Institute on Drug Abuse . 2014.

Ref Type: Online Source

25. Peter Kalix; Olav Braenden Pharmacological Aspects of the Chewing of Khat Leaves. Pharmacological Reviews 1985, 37 (2), 149-164. 
26. Anteneh M.Feyissa; John P.Kelly A review of the neuropharmacological properties of khat. Progress in Neuro-Psychopharmacology and Biological Psychiatry 2008, 32, 1147-1166.

27. Maria Joao Valente; Paula Guedes de Pinho; Maria de Lourdes Bastos; Felix Carvalho; Marcia Carvalho Khat and synthetic cathinones: a review. Arch Toxicol 2013, 88, 15-45.

28. Mike Powers Vegetable to Chemical. In Drugs Unlimited: The Web Revolution That's Changing How the World Gets High, 1 ed.; St. Martin's Press: New York, 2014; pp 1-27.

29. Paul S.Cohen; Stephen M.Cohen Wohler's Synthesis of Urea: How Do the Textbooks Report It? Journal of Chemical Education 1996, 73 (9), 883886.

30. John P.Kelly Cathinone derivatives: A review of their chemistry, pharmacology and toxicology. Drug Testing and Analysis 2011, 3, 439-453.

31. Richard Young; Richard A.Glennon Cocaine-stimulus generalization to two new designer drugs: methcathinone and 4-methylaminorex. Pharmacology Biochemistry and Behavior 1993, 45 (1), 229-231.

32. G.Gardos; J.O.Cole Evaluation of pyrovalerone in chronically fatigued volunteers. Current Therapeutic Research, Clinical and Experimental 1971, 13 (10), 631-635.

33. Peyton Jacob lii; Alexander T Shulgin Novel n-substituted-2-amino-3',3'methylene-dioxypropiophenones. WO 1996039133 Al, Dec 12, 1996.

34. American Association of Poison Control Centers Balt Salts Data; American Assoication of Poison Control Center: 16.

35. National Drug Intelligence Center Synthetic Cathinones (Bath Salts): An Emerging Domestic Threat;2011-S0787-004; U.S. Department of Justice: 11.

36. Joseph LaPointe; Brian Musscleman; Teresa O'Neill; Jason R.E.Shepard Detection of "Bath Salt" Synthetic Cathinone and Metabolites in Urine via DART-MS and Solid Phase Microextraction. Journal American Society for Mass Spectrometry 2015, 26, 159-165.

37. Matthew L.Banks; Travis J.Worst; Daniel E.Rusyniak; Jon E.Sprague Synthetic Cathinones ("Bath Salts"). Journal of Emergency Medicine 2014, 46, 632642 . 
38. Rosenbaum, C.; Carreiro, S.; Babu, K. Here Today, Gone Tomorrow and Back Again? A Review of Herbal Marijuana Alternatives (K2, Spice), Synthetic Cathinones (Bath Salts), Kratom, Salvia divinorum, Methoxetamine, and Piperazines. J. Med. Toxicol. 2012, 8 (1), 15-32.

39. M.Coppola; R.Mondola Synthetic cathinones: Chemistry, pharmacology and toxicology of a new class of designer drugs of aduse marketed as "bath salts" or "plant food". Toxicology Letters 2012, 211, 144-149.

40. Perspectives on Drugs: Injection of synthetic cathinones. European Monitoring Centre for Drugs and Drug Addiction 2015, 1-7.

41. Richard Winton . Redlands bans synthetic drugs "bath salts" and "Spice". Los Angeles Times . 8-15-2013. 7-2-2016.

Ref Type: Online Source

42. Philip Seeman Brain Dopamine Receptors. Pharmacological Reviews 1981, 32 (3), 229-313.

43. Randy D.Blakely; Louis J.De Felice; H.Criss Hartzell Molecular Physiology of Norepinephrine and serotonin Transporters. Journal of Experimental Biology 1996, 196, 263-281.

44. Fabrizio Schifano; Antonio Albanese; Suzanne Fergus; Jackie L.Stair; Paolo Deluca; Ornella Corazza; Zoe Davey; John Corkery; Holger Siemann; Norbert Scherbaum; Magi' Farre; Marta Torrens; Zsolt Demetrovics; A.Hamid Ghodse; Pyschonaut Web Mapping; ReDNet Research Groups Mephedrone (4-methylmethcathinone; 'meow meow'): chemical, pharmacological and clinical issues. Psychopharmacology 2011, 214 (3), 593-602.

45. LD Simmler; TA Buser; M Donzelli; Y Schramm; L-H Dieu; J Huwyler; S Chaboz; MC Hoener; ME Liechti Pharmacological characterization of designer cahtinones in vitro. British Journal of Pharmacology 2012, 168, 458-470.

46. Michael H.Baumann; John S.Partilla; Kurt R.Lehner Psychoactive "bath salts": Not so soothing. European Journal of Pharmacology 2012, 698, 1-5.

47. M.G.Bossong; J.P.Van Dijk; R.J.M.Niesink Methyloen and mCPP, two new drugs of abuse? Addicition Biology 2005, 10, 321-323.

48. Laurent Karila; Michel Reynaud GHB and synthetic cathinones: clinical effects and potential consequences. Drug Testing and Analysis 2010, 3 (9), 552559. 
49. Kristyn Tekulve; Andreia Alexander; Laura Tormoehln Seizures Associated With Synthetic Cathinone Exposures in the Pediatric Population. Pediatric Neurology 2014, 51, 67-70.

50. Penders TM; Gestring RE; Vilensky DA Intoxication delirium following use of synthetic cathinone derivatives. American Journal of Drug Alcohol Abuse 2012, 38 (6), 616-617.

51. Erik W.Gunderson; Matther G.Kirkpatrick; Laura M.Willing; Christopher P.Holstege Intranasal Substituted Cathinone "Bath Salts" Psychosis Potentially Exacerbated by Diphenhydramine. Journal of Addiction Medicine 2013, 7 (3), 153-162.

52. Brandon J.Warrick; John Wilson; Matthew Hedge; Scott Freeman; Karen Leonard; Cynthia Aaron Lethal Serotonin Syndrome After Methylone and Butylone Ingestion. Journal of Medical Toxicology 2011, 8, 65-68.

53. Peter N.Carbone; David L.Carbone; Shaun D.Carstairs; Scott A.Luzi Sudden Cardiac Death Associated With Methylone Use. Americal Journal of Forensic Medicine \& Pathology 2013, 34 (1), 26-28.

54. Ruth A.Carter . Schedules of Controlled Substances: Temporary Placement of 10 Synthetic Cathinones into Schedule I. 79, 4429-4433. 1-28-2014.

Ref Type: Generic Department of Justice: Office of Diversion Control.

55. Craig Anderson Presumptive and Confirmatory Drug Tests. Journal of Chemical Education 2005, 82 (12), 1809-1810.

56. National Law Enforcement and Corrections Technology Center Color Test Reagents/Kits for Preliminary Identification of Drugs of Abuse;0604.01; U.S. Department of Justice: Washington, D.C., Jul, 00.

57. Palm Beach Sheriff Office Jan 20, 2016.

58. Joseph Sherma; Bernard Fried Basic TLC Techniques, Materials, and Apparatus. In Handbook of Thin-Layer Chromatography, 3 ed.; New York, New York, 2005; pp 1-60.

59. Amitava Dasgupta Methodologies for Drug-of-Abuse Testing. In A Health Educator's Guide to Understanding Drugs of Abuse Testing, Amy L.Flagg, Ed; Jones and Barlett Publishers: 2010; pp 73-91.

60. Immunoassay; United Kingdom ed.; Academic Press: 2016.pp. 1-579.

61. The Use of Antibodies in Immunoassays;ML-302-04; SeraCare Life Sciences, 05. 
62. Alternative Immunoassay; A Wiley Medical Publication: 1984.pp. 1-244.

63. Eva Engvall Enzyme immunassay ELISA and EMIT. Methods in Enzymology 1980, 70, 419-439.

64. Sino Biological Solution Specialist . ELISA Encyclopedia. Sino Biological Inc. 2016. 2016.

Ref Type: Online Source

65. Charles F.Solane Dogs in War, Police Work and on Patrol. Journal of Criminal Law and Criminology 1955, 46 (3), 385-395.

66. S.Kris Kawucha K-9 Units and Policing. Telemasp Bulletin 2009, 16 (4), 1-7.

67. Colonel Konrad Most Training Dogs: A Manual; 1 ed.; Dogwise Publishing: 1955.pp. 7-204.

68. Calre Browne; Kevin Stafford; Robin Fordham The use of scent-detection dogs. Irish Veterinary Journal 2006, 59 (2), 97-104.

69. Ron Mistafa K9 Explosive Detection; Detselig Enterprises Ltd.: Calgary, 1998.pp. 9-189.

70. William H.Whitstine Jr. Sniffing the Ashes K-9's in the Fire Service; International Society of Fire Service Instructors: 1992.pp. 1-79.

71. Adee Schoon; Ruud Haak K9 Suspect Discrimination; Detselig Enterprises Ltd.: Calgary, 2002.pp. 7-165.

72. William G.Syrotuck Scent and the Scenting Dog; 4 ed.; Barkleigh Productions Inc: Mechanicsburg, 2000.pp. 1-112.

73. Kenneth G.Furton; Ross J.Harper Controlled Odor Mimic Permeation System. FL/US Dec 4, 2008.

74. Adam Cole . The Latest In Scientific Field Equipment? Fido's Nose. 1-11. 9-32013. National Public Radio.

Ref Type: Generic

75. Tadeusz Jezierski; Ewa Adamkiewicz; Marta Walczak; Magdalena Sobczynska; Aleksandra Gorecka-Bruzda; John Ensminger; Eugene Papet Efficacy of drug detection by fully-trained police dogs varies by breed, training level, type of drug and search environment. Forensic Science International 2014, 237 (2014), 112-118. 
76. Kenneth G.Furton; Jessie Greb; Howard Holness The Scientific Working Group on Dog and Orthogonal Detector Guidelines (SWGDOG);231952; U.S. Department of Justice: Sep, 10.

77. Kenneth G.Furton; Jessie Greb; Howard Holness . SWGDOG SC2: General Guidelines. Scientific Working Group for Dog and Orthogonal Guidelines [1], 1-6. 12-16-2006.

Ref Type: Online Source

78. Kenneth G.Furton; Jessie Greb; Howard Holness . SWGDOG SC8- Substance Detector Dogs Narcotics Section. Scientific Working Group for Dog and Orthogonal Guidelines, 1-6. 5-1-2007. Miami.

Ref Type: Online Source

79. Stacia Glenn Tacoma police dog dies after inhaling meth. The News Tribune, Mar 26, 2015, pp 1-5.

80. Sigma-Aldrich . Canine Training Aids, Sigma Pseudo Scents. 2016. SigmaAldrich Co.

Ref Type: Online Source

81. Lawrence Livermore . Non-Hazardous Explosives for Security Training and Testing (NEST). Lawrence Livermore National Laboratory . 2000. California.

Ref Type: Online Source

82. Michael S.Macias; Patricia Guerra-Diaz; Jose R.Almirall; Kenneth G.Furton Detection of piperonal emiited from polymer controlled odor mimic permeation systems utilizing Canis familiaris and solid phase microextraction-ion mobility spectrometry. Forensic Science International 2009, 195, 132-138.

83. Zhouyao Zhang; Janusz Pawlisyzn Headspace Solid-Phase Microextraction. Analytical Chemistry 1993, 65 (14), 1843-1852.

84. Solid Phase Microextraction: A Practical Guide; Marcel Dekker AG: Basel, 1999.pp. 1-239.

85. Gyorgy Vas; Karoly Vekey Solid-phase microextraction: a powerful sample preparation tool prior to mass spectrometric analysis. Journal of Mass Spectrometry 4 A.D., 39, 233-254.

86. Sigma-Aldrich . Selection Guide for Supelco SPME Fibers. Sigma-Aldrich Co. 1-3. 2016.

Ref Type: Generic 
87. Michael P.DiCicco; Bridget Lang; Thomas I.Harper Optimization of a novel headspace-solid-phase microextraction-gas chromatographic method by means of a Doehlert uniform shell design fro the analysis opf trace level ethylene oxide residuals in sterilized medical devices. Biomedical Chromatography 2009, 23, 647-657.

88. Amy M.Dixon; Stacy D.Brown; S.Muralidhara; James V.Bruckner; Michael G.Bartlett Optimization of SPME for Analysis of Trichloroethylene in Rat Blood and Tissues by SPME-GC/MS. Instrumentation and Science and Technology 2004, 33, 175-186.

89. K.Robards; P.R.Haddad; P.E.Jackson Pricinples and Practice of Modern Chromatographic Methods; Academic Press An Imprint of Elsevier Science: London, 2002.pp. 1-491.

90. C.Simpson Gas Chromatography; 1 ed.; Kogan Page: England, 1970.pp. 7-117.

91. D.Ambrose Gas Chromatography; 2 ed.; The Butterworth Group: England, 1971.pp. 1-321.

92. Choosing the right column. 1-3. 2016. Agilent Technicologies. Ref Type: Generic

93. Harold McNair. Introduction to GC. Chromedia Analytical Sciences [1], 1-3. 2016.

Ref Type: Online Source

94. Harold McNair; James M Miller Basic Gas Chromatography; John Wiley \& Sons Inc: 1998.pp. 1-104.

95. Dhanlal De Lloyd . Gas Chromatography Detectors. J\&W Catalogue "Gas Chromatography" 1998 . 2000. The Republic of Trindad and Tobago, The Univeristy of The West Indies.

Ref Type: Online Source

96. Edmond de Hoffmann; Vincent Stroobant Mass Spectrometry Principles and Applications; 3 ed.; John Wiley \& Sons Ltd.: England, 2007.pp. 9-350.

97. A.G.Harrison Chemical Ionization Mass Spectrometry; CRC Press: 1992.

98. M.S.B.Munson; F.H.Field Chemical Ionization Mass Spectrometry. Journal of the American Chemical Society 1966, 88 (12), 2621-2630.

99. Burnaby Munson Chemical Ionization Mass Spectrometry. Analytical Chemistry 1971, 43 (13), 28-34. 
100. Varian . Saturn 2000 GC/MS: MS Workstation. 155-167. 2003. Quality System ISO 9001 Certified.

Ref Type: Generic

101. Stan Gibilisco; Margaret Rouse . Statistical Analysis. Business intelligencebusiness analytics , 1-3. 2014. 10-12-2016.

Ref Type: Online Source

102. James N Miller; Jane C.Miller Statistics and Chemometrics for Analytical Chemistry; Pearson Education Limited: England, 2010; Vol. 6;.pp. 1-297.

103. Parikh R; Mathai A; Parikh S; Chandra Sekhar G; Thomas R Understanding and using sensitivity, specificity and predictive values. Indian J Ophthalmol 2008, 56 (1), 45-50.

104. Wayne W.LaMorte . Positive and Negative Predictive Values. Boston University School of Public Health . 6-15-2016. 10-12-2016.

Ref Type: Online Source

105. Kathryn A.Seely; Amy L.Patton; Cindy L.Moran; Mary L.Womack; Paul L.Prather; William E.Fantegrossi; Anna Radominska-Pandya; Gregory W.Endres; Kermit B.Channell; Nathaniel H.Smith; Keith R.McCain; Laura P.James; Jeffery H.Moran Forensic investigation of K2, Spice, and "bath salt" commercial preparations: A three-year study of new designer drug products containing synthetic cannabinoid, stimulant, and hallucinogenic compounds. Forensic Science International 2013, 233, 416-422.

106. Kathleen Meyers; Ovgu Kaynak; Elena Bresani; Brenda Curtis; Ashley McNamara; Kristine Brownfield; Kimberly C.Kirby The availability and depiction of synthetic cathinones (bath salts) on the Internet: Do online suppliers employ features to maximize purchases? International Journal of Drug Policy 2015, 26, 670-674.

107. Jeffery Hackett; Kerrie T.Donovan; Albert A.Elian Analysis of Amphetamines and Synthetic Cathiones in Hair Samples Using LC-Tandem Mass Spectrometry and Solid-Phase Extraction. Liquid Chromatography and Gas Chromatography: Solutions for Separation Scientists, 2014, pp 1-5.

108. Kenji Tsujikawa; Kenji Kuwayama; Tatsuyuki Kanamori; Yuko T.Iwata; Hiroyuki Inoue Thermal degradation of a-pyrrolidinopentiophenone during injection in gas chromatography/mass spectrometry. Forensic Science International 2013, 231, 296-299. 
109. Sarah Kerrigan; Megan Savage; Cassandra Cavazos; Paige Bella Thermal Degradation of Synthetic Cathinones: Implications for Forensic Toxicology. Journal of Analytical Toxicology 2016, 40, 1-11.

110. Ashton D.Lesiak; Rabi A.Musah; Robert B.Cody; Marek A.Domin; A.John Dane; Jason R.E.Shepard Direct analysis in real time mass spectrometry (DARTMS) of "bath salt" cathinone drug mixtures. The Royal Society of Chemistry 2013, 138, 3424-3432.

111. Justin L.Poklis; Carl E.Wolf; Omar I.Eljordi; Kai Liu; Shijun Zhang; Alphonse Poklis Analysis of the First- and Second-Generation Raving Dragon Novelty Bath Salts Containing Methylone and Penedrone*. Journal of Forensic Sciences 2014, 60, 234-240.

112. Federica Bonadio; Pierre Margot; Olivier Delemont; Pierre Esseiva Optimization of HS-SPME/GC-MS analysis and its use in the profiling of illicit ecstasy tablets (Part 1). Forensic Science International 2009, 187, 73-80.

113. Alvin C.Bronstein; Daniel A.Spyker; Louise R.Cantilena Jr.; Barry H.Rumack; Richard C.Dart 2011 Annual Report of the American Association of Poison Control Centers' National Poison Data System (NPDS): 29th Annual Report; 12.

114. Doan-Trang T.Vu SPME/GC-MS Characterization of Volatiles Associated with Methamphetamine: Toward the Devleopment of a Pseudomethamphetamine Training Material. Journal of Forensic Sciences 2000, 32 (19), 1014-1024. 


\section{APPENDICES}

Table 32. Chemical Ionization (Acetonitrile) Parameter Modification of Background Mass (BM)

\begin{tabular}{|c|c|c|c|c|c|c|}
\hline Method Name & $\begin{array}{c}\text { Storage } \\
\text { Level }\end{array}$ & $\begin{array}{c}\text { Ejection } \\
\text { Amp }\end{array}$ & $\mathbf{B M}$ & TIC & $\underset{\text { IT }}{\operatorname{Max}}$ & $\begin{array}{c}\text { Max } \\
\text { RT }\end{array}$ \\
\hline Acetonitrile Method2 & 19 & 18 & 65 & 5000 & 2000 & 40 \\
\hline Acetonitrile Method10 & 19 & 18 & 70 & 5000 & 2000 & 40 \\
\hline Acetonitrile Method11 & 19 & 18 & 75 & 5000 & 2000 & 40 \\
\hline Acetonitrile Method12 & 19 & 18 & 60 & 5000 & 2000 & 40 \\
\hline Acetonitrile Method13 & 19 & 18 & 55 & 5000 & 2000 & 40 \\
\hline Acetonitrile Method14 & 19 & 18 & 50 & 5000 & 2000 & 40 \\
\hline
\end{tabular}

Table 33. Chemical Ionization (Acetonitrile) Parameter Modification of Maximum Ionization Time (Max IT)

\begin{tabular}{|c|c|c|c|c|c|c|}
\hline Method Name & $\begin{array}{c}\text { Storage } \\
\text { Level }\end{array}$ & $\begin{array}{c}\text { Ejection } \\
\text { Amp }\end{array}$ & $\mathbf{B M}$ & TIC & $\begin{array}{c}\text { Max } \\
\text { IT }\end{array}$ & $\begin{array}{c}\text { Max } \\
\text { RT }\end{array}$ \\
\hline Acetonitrile Method2 & 19 & 18 & 65 & 5000 & 2000 & 40 \\
\hline Acetonitrile Method15 & 19 & 18 & 65 & 5000 & 2500 & 40 \\
\hline Acetonitrile Method16 & 19 & 18 & 65 & 5000 & 1500 & 40 \\
\hline Acetonitrile Method17 & 19 & 18 & 65 & 5000 & 1000 & 40 \\
\hline Acetonitrile Method18 & 19 & 18 & 65 & 5000 & 500 & 40 \\
\hline
\end{tabular}




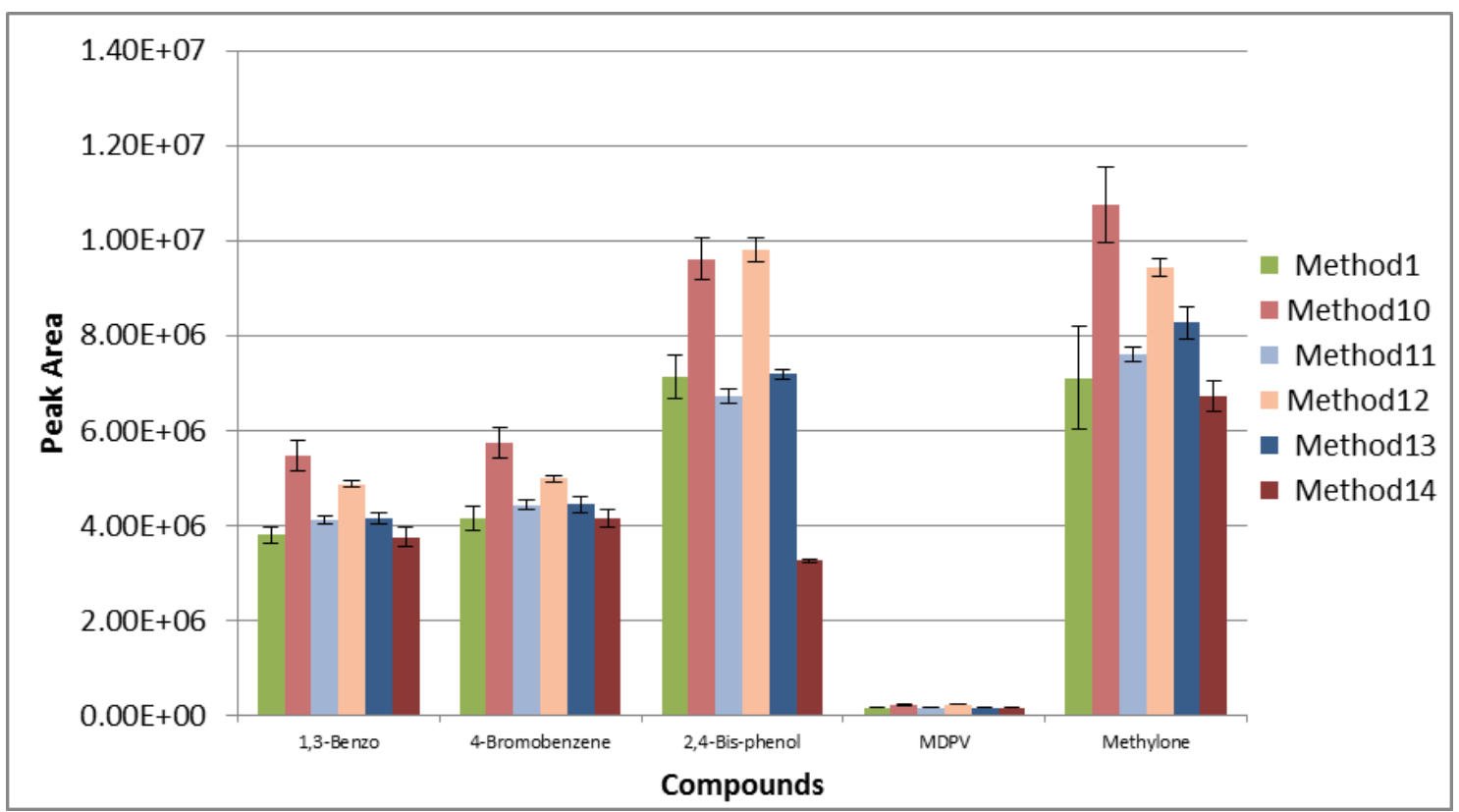

Figure 69. CI-Methanol Background Mass Parameter Optimization

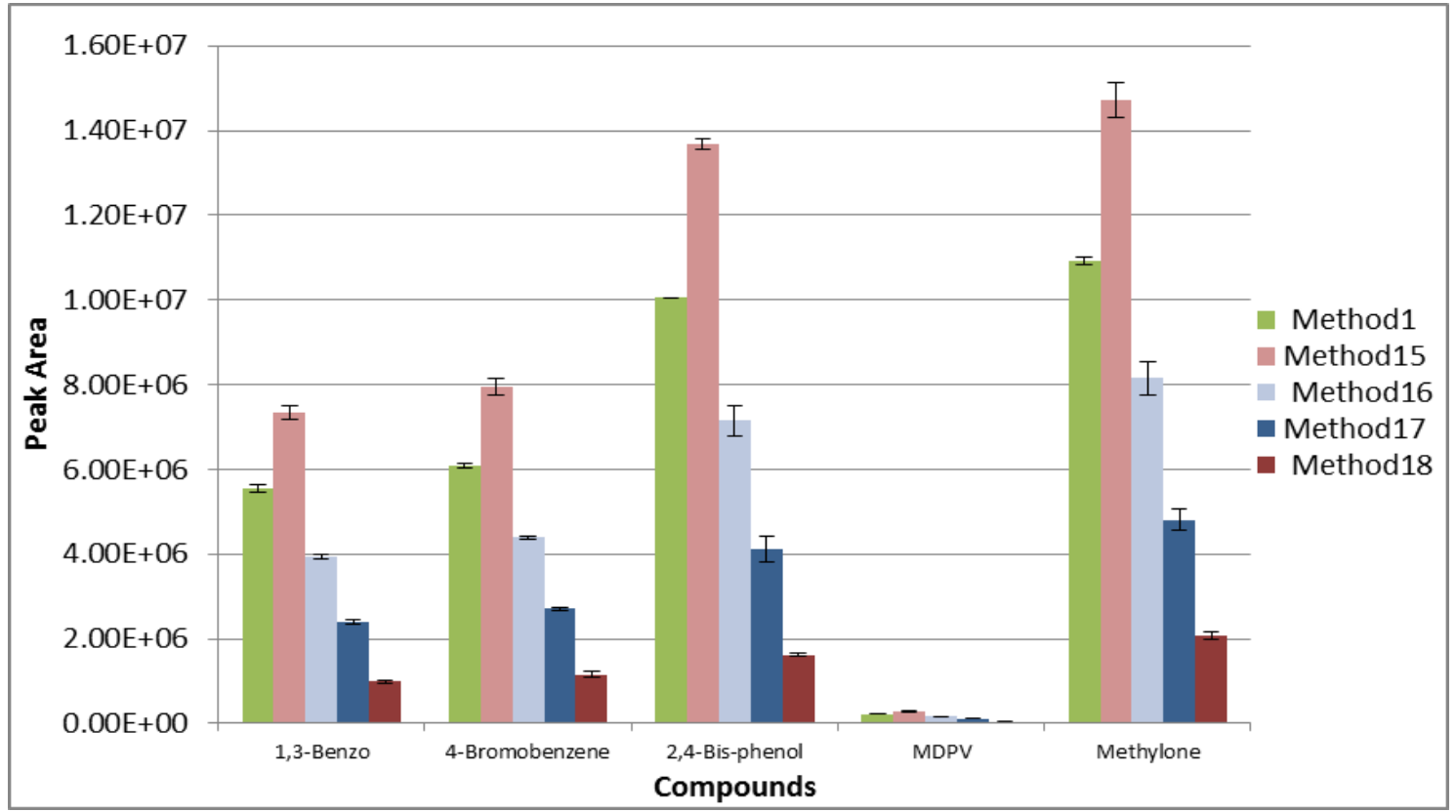

Figure 70. CI-Methanol: Maximum Ionization Time Parameter Optimization 


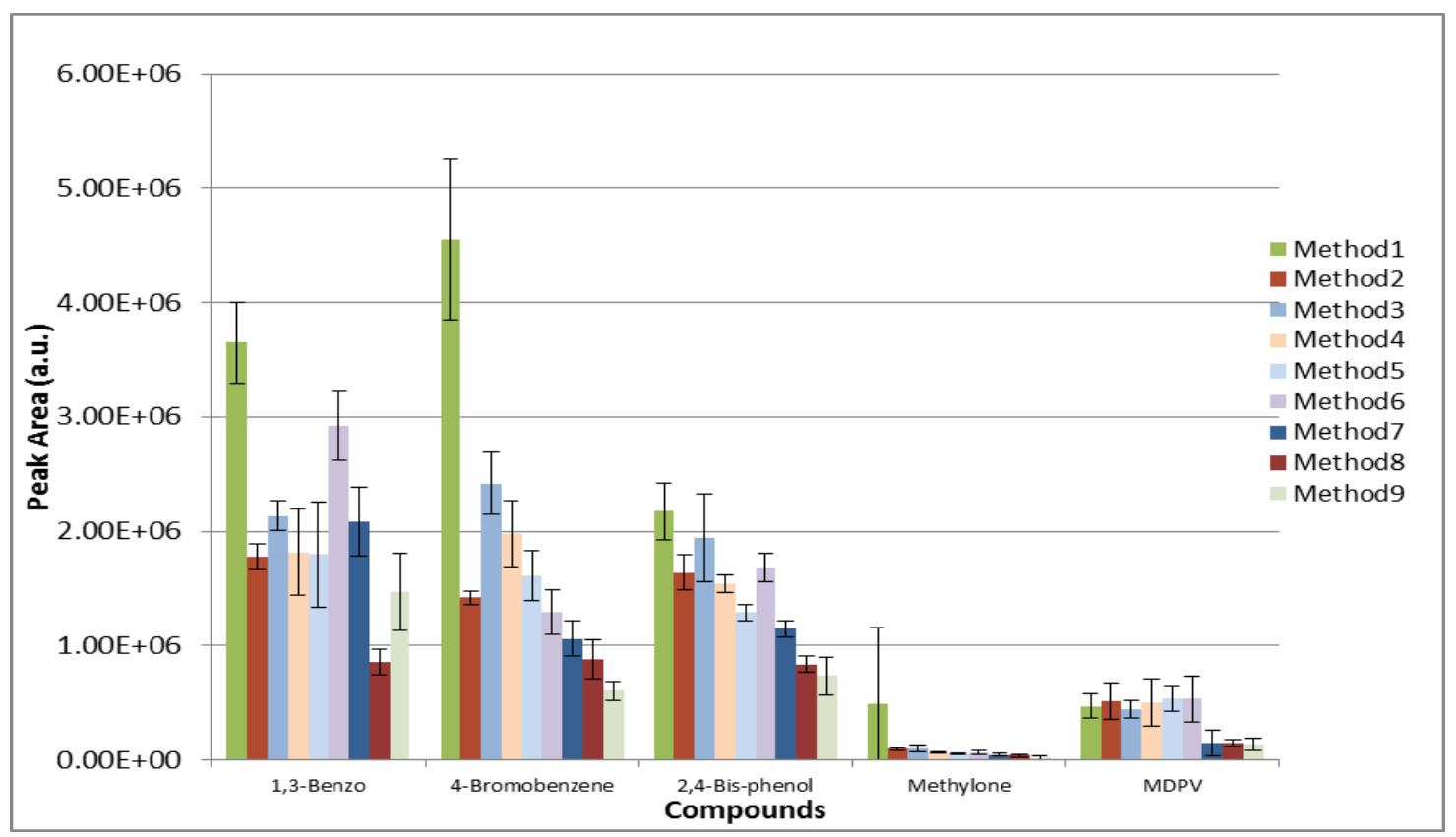

Figure 71. CI-Acetonitrile: Storage Level and Ejection Amplitude Parameter Optimization

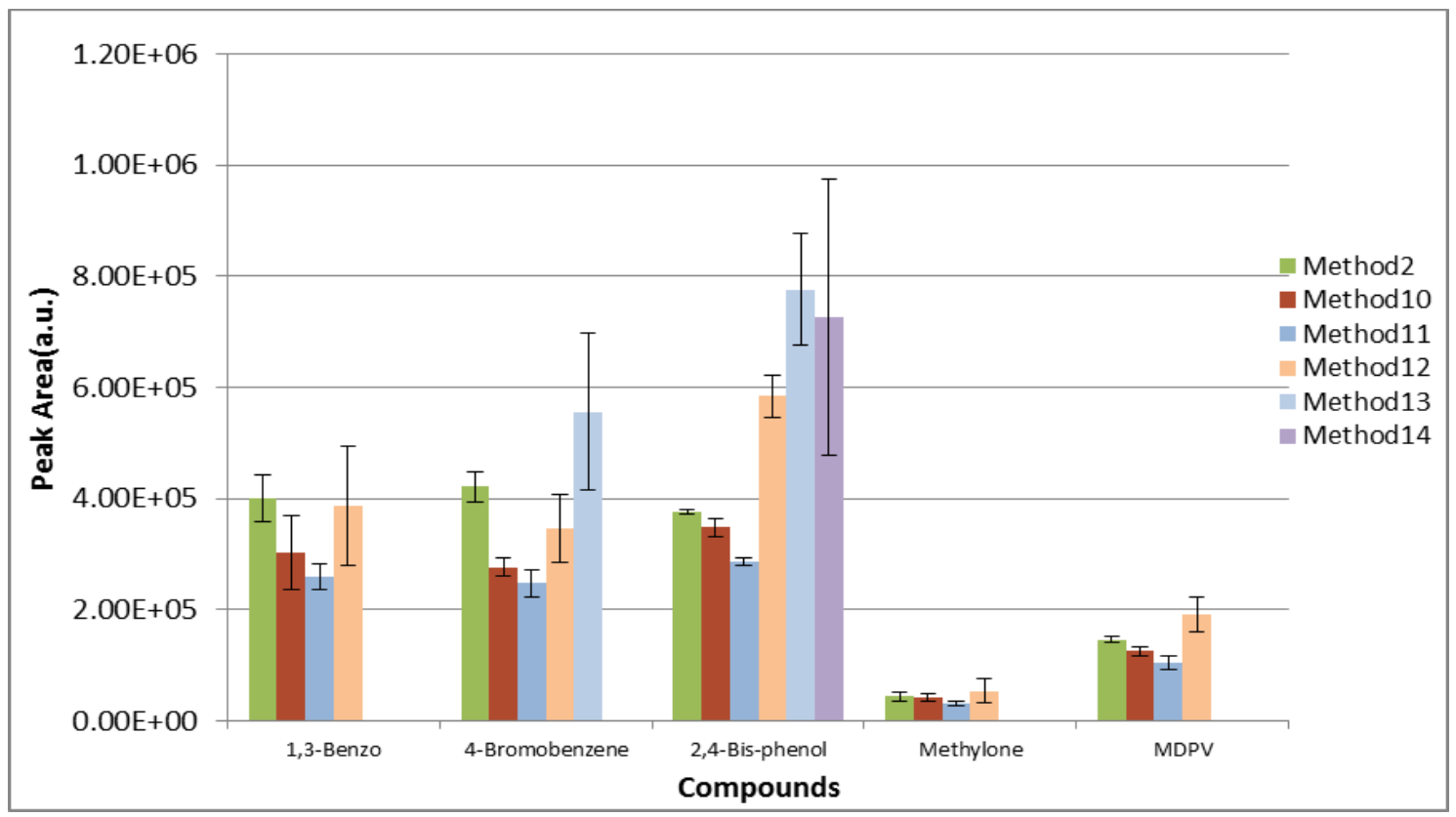

Figure 72. CI Acetonitrile: Background Mass Parameter Optimization 


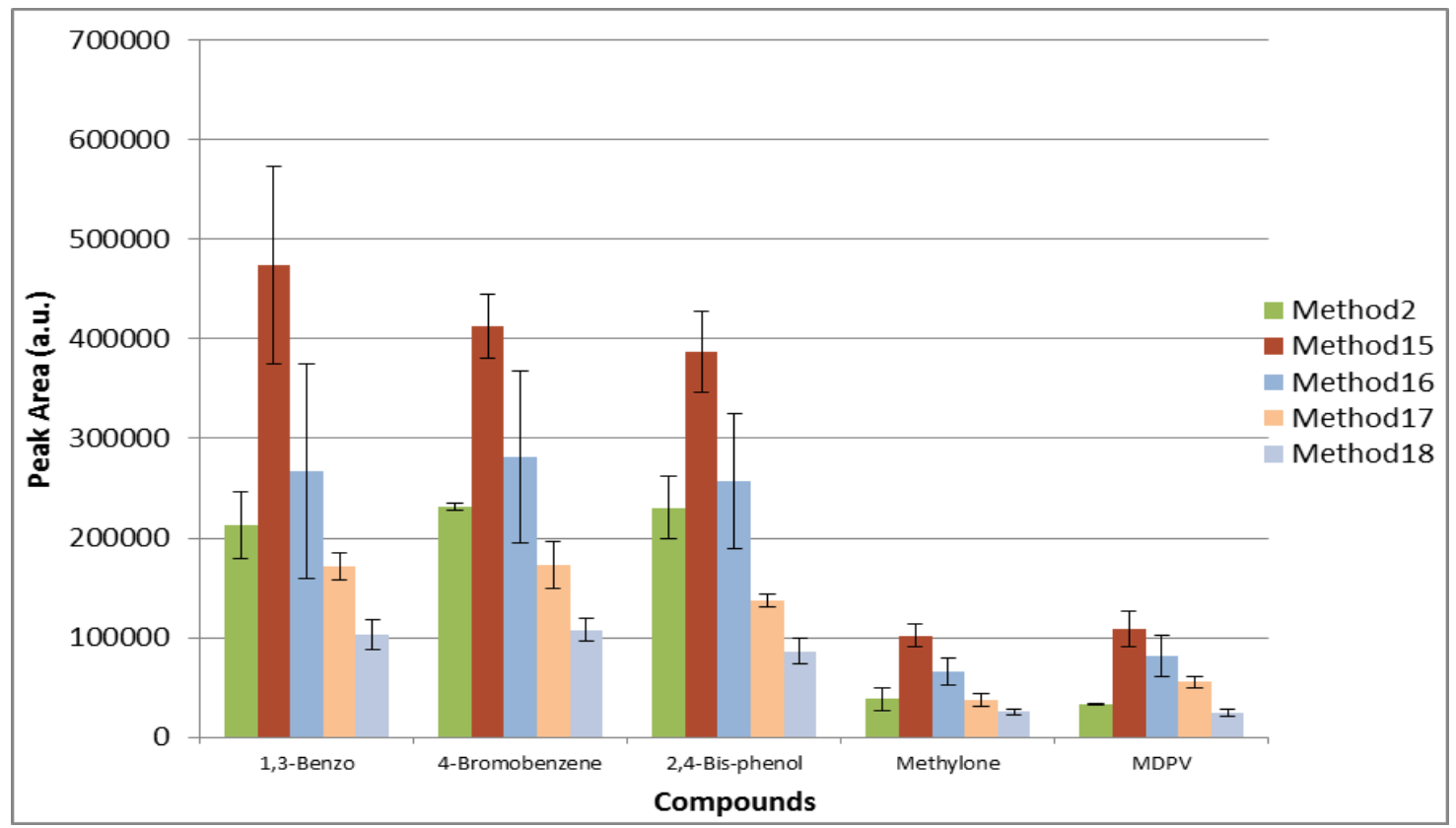

Figure 73. CI-Acetonitrile: Maximum Ionization Time Parameter Optimization 
Certification results for:

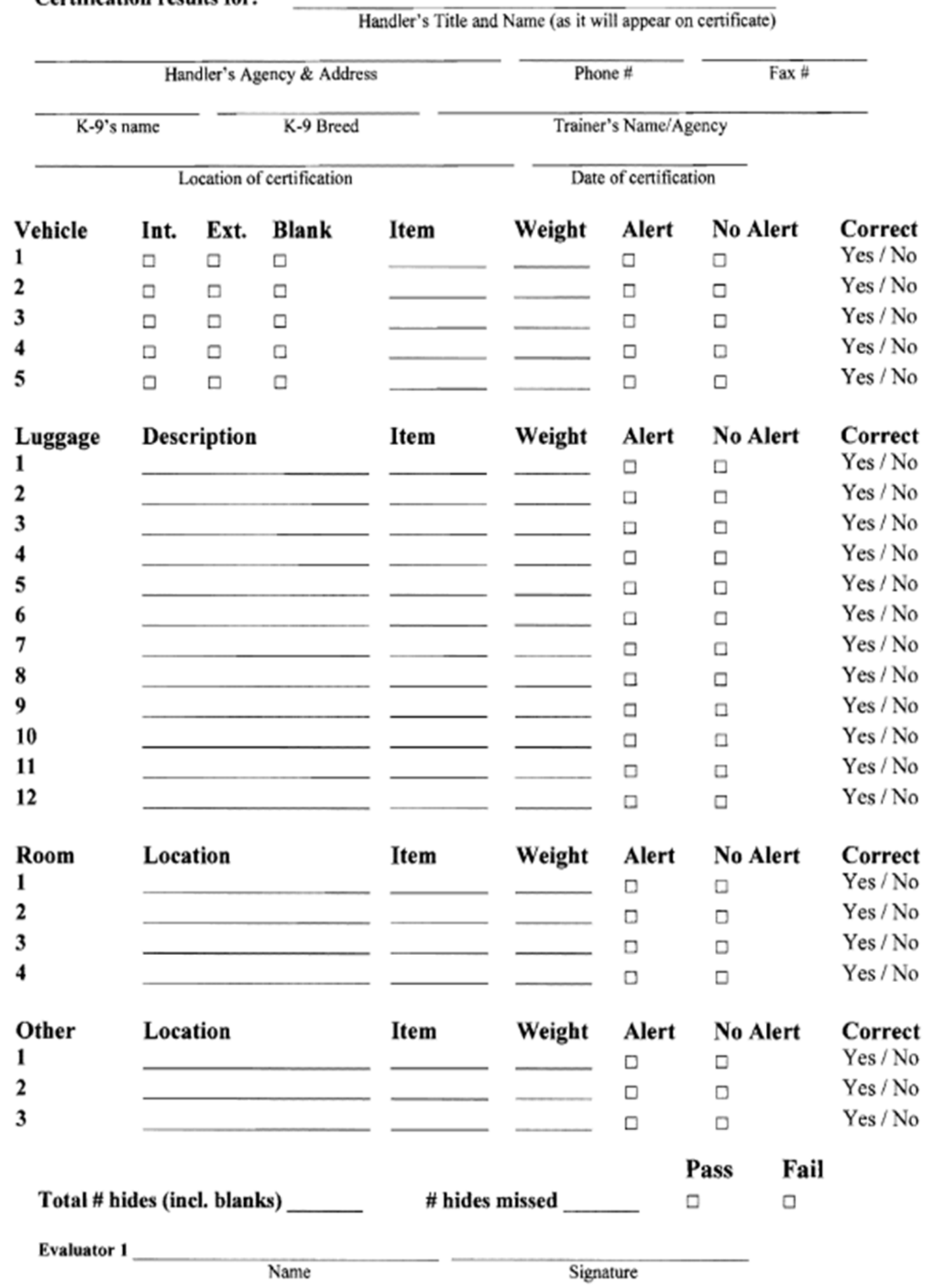

Figure 74. Canine Odor Recognition Evaluation Form 


\section{VITAE}

\section{VANQUILLA SHELLMAN}

July 13, 1990

2004-2008

2008-2012

2010-2012

2010-2012

2012-2017

2012-2016

2013-2017
Born Orlando, Florida

High School Diploma (Salutatorian)

Liberty High School

Kissimmee, Florida

Bachelors of Arts in Chemistry (Magnum Cum Laude) Florida International University

Miami, Florida

McNair Fellow

Florida International University

Miami, Florida

Undergraduate Research Assistant

Florida International University

Miami, Florida

Research/ Teaching Assistant

Florida International University

Miami, Florida

Masters Science in Forensic Science

Florida International University

Miami, Florida

McKnight Doctoral Fellow

Florida International University

Miami, Florida

Palm Beach Office Intern

Palm Beach Sheriff Office

West Palm Beach, FL 


\section{PUBLICATIONS AND PRESENTATIONS}

V. Shellman and K.G. Furton. Optimal Headspace Extraction for the Detection of Volatile Organic Compounds (VOCs) Released from Synthetic Cathinones using Solid Phase Micro-Extraction (SPME) Forensic Science International. Submitted

V. Shellman, H. Holness, K.G. Furton. An Assessment of Different Extraction Techniques for the Detection of Odorants Released from Synthetic Cathinone Derivatives. AAFS conference, Seattle, Washington. 2014.

V. Shellman, H. Holness, K.G. Furton. An Assessment of Different Extraction Techniques for the Detection of Odorants Released from Synthetic Cathinone Derivatives. IFRI Conference, Miami, Florida, 2014.

V. Shellman and K.G. Furton. Optimal Headspace Extraction for the Detection of Volatile Organic Compounds (VOCs) Released from Synthetic Cathinones using Solid Phase Micro-Extraction (SPME). FAME Conference, Tampa, Florida, 2015.

V. Shellman and K.G. Furton. Optimal Headspace Extraction Method for the Detection of Volatile Organic Compounds (VOCs) Released from Synthetic Cathinones using Solid Phase Micro-Extraction (SPME) and its Field Application. AAFS Conference, New Orleans, Louisiana, 2017.

V. Shellman and K.G. Furton. Determination of the Active Odorant for Canine Detection of Synthetic Cathinones for the Development of a Controlled Mimic Permeation System (COMPS). IWD Conference, Banff, Canada, 2017

V. Shellman, A. Simon, A. Boone, K.G. Furton. The Development and Understanding of Efficient Mimic Devices for Calibrating and Training Detection Canines for Forensic, Environmental, and Medical Use. IWD Conference, Banff, Canada, 2017 